\title{
Estrategia de búsqueda y
} optimización de parámetros con aplicación en la simulación mediante códigos termohidráulicos

TESIS D OCTORAL

Presentada por: Sofia Carlos Alberola

Dirigida por: Dr. I.I. Sebastián Martorell Alsina Dr. I.I. Vicente Serradell G arcia 



\section{RESUMEN}

La evaluación del comportamiento de las plantas nucleares ante un escenario transitorio es una línea de trabajo dentro del campo de la seguridad nuclear desde que empezó la explotación de la energía nuclear para la producción de energía eléctrica. No obstante, al igual como ocurre en muchos otros ámbitos de la ingeniería, la comprobación experimental del comportamiento de las centrales nucleares ante diferentes situaciones no es viable en la mayoría de los casos. Esto hace necesario disponer de herramientas de simulación de secuencias accidentales de manera que se puedan extrapolar los resultados a las instalaciones reales.

En este sentido, los organismos reguladores permiten utilizar códigos de simulación termohidráulica para garantizar la seguridad de las instalaciones siempre que se cuantifique la incertidumbre asociada a simulación. Las incertidumbres asociadas a una simulación tienen diversos orígenes entre los que se encuentran la limitación de los modelos físicos utilizados a la hora de describir los distintos fenómenos físicos que tienen lugar en la planta, los métodos numéricos utilizados por los códigos para la resolución de estos fenómenos y los valores de diferentes variables que resultan desconocidos al realizar el modelo de la instalación.

Para cuantificar y acotar estas incertidumbres se han propuesto varias metodologías, sin embargo, todas ellas necesitan de la realización de diversos análisis de sensibilidad que se realizan manualmente $\mathrm{y}$, por tanto, dependen en gran medida del usuario que realiza el análisis.

En esta tesis se ha desarrollado y aplicado una nueva metodología para la cuantificación de las incertidumbres de una simulación que hace uso de técnicas de búsqueda y optimización con el fin de minimizar el efecto de usuario que se introduce en las simulaciones termohidráulicas. Así, se presenta un método automático y sistemático de búsqueda y optimización de parámetros termohidráulicos, de manera que se asegura que el error cometido es el mínimo posible. Esta nueva metodología se ha aplicado a modelos sencillos como la ecuación de la convección y la ecuación de la conveccióndifusión mediante dos técnicas de optimización con filosofías diferentes como son un algoritmo genético y un algoritmo de búsqueda directa. En particular se ha utilizado un algoritmo genético de estado estacionario y el método de búsqueda multidireccional. Tras los estudios realizados se concluye que el método de búsqueda directa es más eficiente para el tratamiento de este tipo de problemas. 
Como aplicación a un código termohidráulico de estimación óptima se ha hecho un análisis de dos casos incluidos en la matriz de valoración del código RELAP5. En concreto, con estas aplicaciones se ha comprobado la capacidad de la metodología en la determinación de valores óptimos de parámetros del modelo de las instalaciones como son las condiciones iniciales y las condiciones de contorno, que en muchas simulaciones son desconocidas.

Por último, se ha mostrado la utilidad de la metodología desarrollada para la construcción de modelos reducidos de reactor. Estos modelos presentan una serie de parámetros efectivos que se pueden optimizar para que el comportamiento del modelo reducido se asemeje lo más posible al de la planta que representa. En particular, se ha construido un modelo reducido de reactor PWR con dos lazos asimétricos y se han determinado algunos de sus parámetros efectivos mediante la simulación de transitorios de operación, utilizando como referencia los cálculos proporcionados por el código de estimación óptima RELAP5. El modelo reducido con los parámetros obtenidos tras la optimización permite predecir con una buena precisión el comportamiento del reactor para estos transitorios. 


\section{RESUM}

L'avaluació de comportament de les plantes nuclears davant d'un escenari transitori és una línia de treball dins del camp nuclear que va sorgir des del començament de l'explotació de l'energia nuclear per a la producció d'energia elèctrica. Malgrat tot, de la mateixa manera a com succeeix en molts altres àmbits de l'enginyeria, la comprovació experimental del comportament de les centrals nuclears davant de diferents situacions accidentals no és viable en la majoria dels casos. Açò, fa necessària la utilització de ferramentes de simulació de seqüències accidentals de manera que els resultats es puguen extrapolar a les instal-lacions reals.

En aquest sentit, els organismes reguladors permeten l'ús de codis de simulació termohidràulica per a garantir la seguretat de les instal-lacions sempre que es quantifique la incertesa associada a la simulació. Les incerteses associades a una simulació tenen diversos orígens entre els que es troben la limitació dels models físics utilitzats per a descriure els diferents fenòmens físics que tenen lloc en la planta, els mètodes numèrics utilitzats per els codis per a la resolució d'aquestos fenòmens $\mathrm{i}$ els valors de diferents variables que són desconegudes quan es realitza un model de la instal·lació.

Per a quantificar i acotar aquestes incerteses s'han proposat diverses metodologies, malgrat tot, totes elles necessiten de la realització de diverses anàlisis de sensibilitat que es realitzen manualment $i$, per tant, depenen en gran mesura de l'usuari que realitza l'anàlisi.

En aquesta tesi s'ha desenvolupat i aplicat una nova metodologia per a la quantificació de les incerteses d'una simulació que fa ús de tècniques de recerca i optimització amb la finalitat de minimitzar l'efecte d'usuari que s'introdueix en les simulacions termohidràuliques. Així, es presenta un mètode automàtic i sistemàtic de recerca $\mathrm{i}$ optimització de paràmetres termohidràulics, de manera que s'assegura que l'error comès és el mínim possible. Aquesta nova metodologia s'ha aplicat a models senzills com ho són l'equació de la convecció i l'equació de la convecció-difusió mitjançant dues tècniques d'optimització de filosofies diferents com ho són els algoritmes genètics i els mètodes de recerca directa. En particular, s'ha utilitzat una algoritme genètic d'estat estacionari i el mètode de recerca multidireccional. Després de la realització d'aquestos estudis es conclou que el mètode de recerca directa és més eficient per al tractament d'aquest tipus de problemes. 
Com a aplicació a un codi termohidràulic d'estimació òptima s'ha realitzat una anàlisi de dos casos inclosos dins la matriu de validació codi RELAP5. Concretament, amb aquestes aplicacions s'ha comprovat la capacitat de la metodologia en la determinació dels valors òptims per a paràmetres dels models de les instal-lacions com ho són les condicions inicials i les condicions de contorn, les quals en moltes simulacions resulten desconegudes.

Finalment, s'ha demostrat la utilitat de la metodologia desenvolupada per a la construcció de models reduïts de reactor. Aquestos models presenten una sèrie de paràmetres efectius que es poden optimitzar per a que el comportament del model reduït s'assemble el més possible al de la planta que representa. En particular, s'ha construït un model reduït d'un reactor PWR amb dos llaços asimètrics, i s'han determinat alguns dels seus paràmetres efectius mitjançant la simulació de transitoris d'operació, utilitzant com a referència els càlculs proporcionats pel codi termohidràulic RELAP5. El model reduït amb els paràmetres obtinguts amb el procés d'optimització permet preveure amb una bona precisió el comportament del reactor per a aquestos transitoris. 


\begin{abstract}
Since the beginning of the utilisation of nuclear energy to generate electric power, the evaluation of the nuclear plants behaviour under accidental scenarios is an important line of research in the field of nuclear safety. But in most cases, as it is the case of other engineering fields, the experimental assessment of nuclear power plants behaviour is not feasible. This reason makes necessary to use simulation tools capable to reproduce the plant behaviour.
\end{abstract}

In this way, the regulatory bodies allow the use of termalhydraulic simulation codes to guarantee the safe operation of the installations, but only if the uncertainty associated with the simulation is properly quantified. The uncertainties associated with a termohydraulic simulation have different origins as: the limitations of the models used to describe the physical process that take place inside the plant, the errors associated with the numerical models used by the codes in the resolution of such models, and unknown values of different variables necessaries to build the plant model.

In order to quantify and limit those uncertainties several methodologies have been developed. But all of them require a certain amount of sensitivity analysis, which are manually performed, and so they strongly depend on the analyst.

In this $\mathrm{PhD}$ thesis a new methodology to quantify and limit the termalhydraulic simulations has been developed. This new methodology uses an optimisation technique to minimise the user effect introduced in the thermalhydraulic simulations. In this way, an automatic and systematic parameter search and optimisation method is proposed to be used to assure that the error introduced in the simulation is the minimum possible. This new methodology is applied to simple models as the convection equation and the convection-diffusion equation using two different optimisation techniques: a genetic algorithm and a direct search method. In particular, a steady state genetic algorithm and the multidirectional search algorithm have been used to perform the analysis. From the results obtained in the analysis it can be concluded that the direct search method is more efficient to deal with this kind of problems.

As an application of the proposed methodology to a best estimated code, two cases included in the RELAP5 assessment matrix have been studied. The purpose of these applications has been to prove the capability of the methodology in the determination of optimal values for parameters of the plant model, such as initial conditions and boundary conditions which are frequently unknown in this kind of simulations. 
Finally, the methodology has also proved to be useful in the construction of reduced reactor models. These models present different effective parameters which can be optimised to achieve a behaviour of the reduced model as similar as possible to the behaviour of the plant that represents. In particular, a two asymmetric loops PWR reactor model has been constructed, and some of its effective parameters have been determined using as a reference the simulations of operational transients performed with the best estimate code RELAP5. It is shown that for such transients, the reduced model using the parameters obtained from the optimisation allows to forecast the behaviour of the plant with good accuracy. 
1. Introducción 1

1.1. Antecedentes

1.2. Objetivos

1.3. Organización del trabajo $\ldots \ldots$

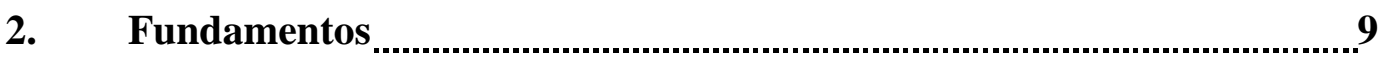

2.1. Códigos termohidráulicos _..................................

2.2. Metodología de cálculo de incertidumbres _...................................

2.2.1. CSAU 16

3. Metodología de búsqueda y optimización de parámetros ….................. 21

3.1. Planteamiento y solución del problema _................................. 21

3.2. Algoritmo Genético.............................................................

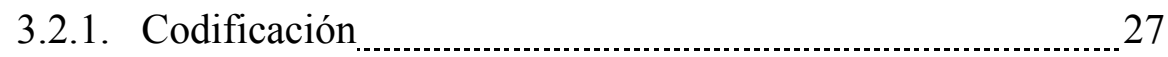

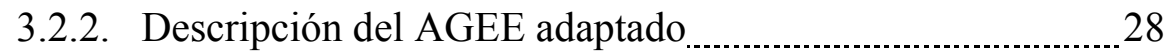

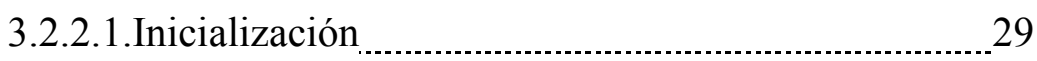

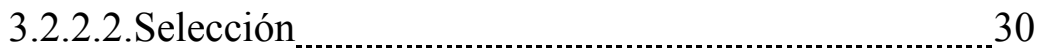

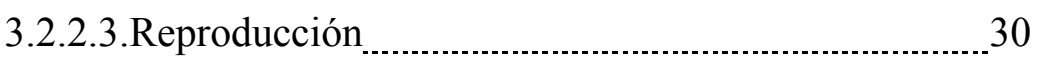

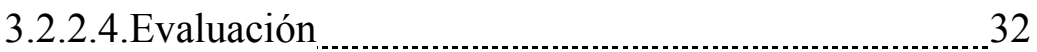

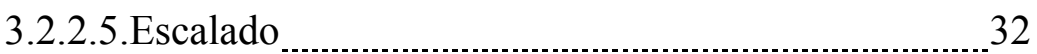

3.2.2.6.Reemplazamiento _........................................... 33

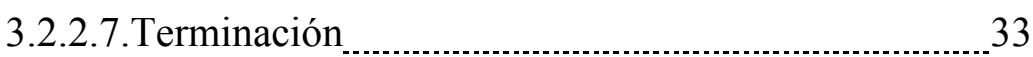

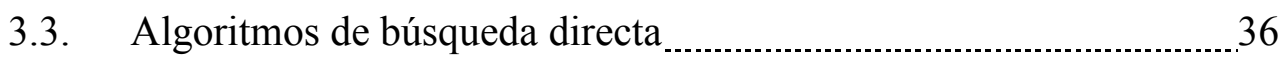

3.3.1. Descripción del algoritmo de búsqueda multidireccional (MDS)

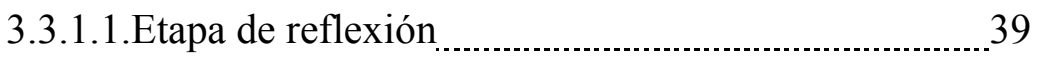

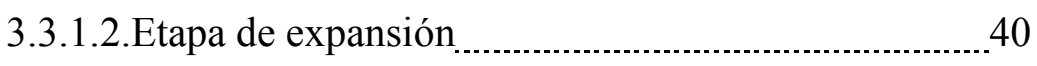

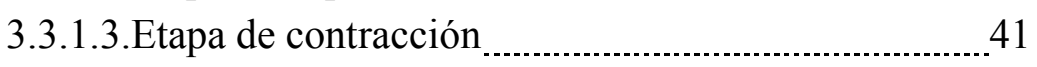

3.3.2. Nuevas direcciones de búsqueda y longitudes de pasos ........ 43

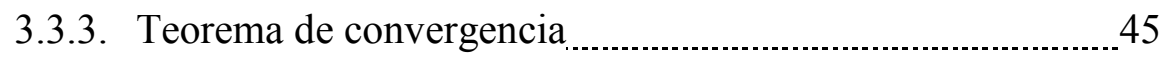

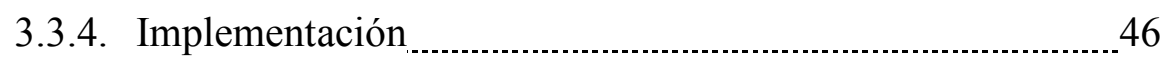

4. Aplicación a modelos termohidráulicos sencillos ....................................49

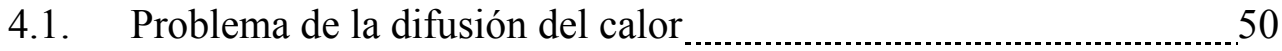

4.1.1. Ecuación de la difusión _............................................ 51

4.1.2. Solución analítica ............................................................ 51

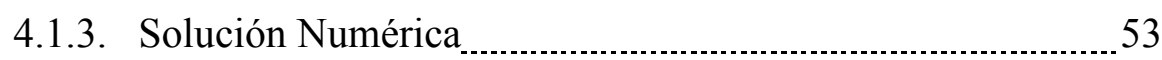

4.1.4. Optimización del coeficiente de difusividad y estudios de sensibilidad 
4.2. Problema de la convección-difusión del calor _......................... 68

4.2.1. Ecuación de convección-difusión unidimensional__............ 69

4.2.2. Solución analítica _....................................... 69

4.2.3. Solución numérica 77

4.2.4. Optimización de parámetros y estudios de sensibilidad ....... 74

5. Aplicación a simulaciones mediante RELAP5/MOD3.2 .......................83

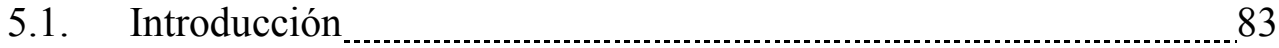

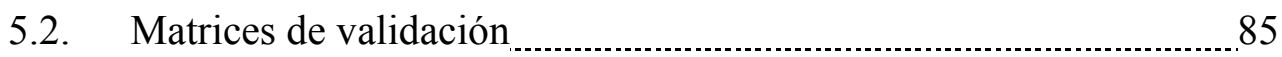

5.3. Edward's pipe $\quad 89$

5.3.1. Descripción del modelo de RELAP5

5.3.2. Simulación y optimización de parámetros _......................... 91

5.4. Royal Institute of Technology (RIT) _.........................................

5.4.1. Descripción de la instalación _.........................................

5.4.2. Descripción del modelo de RELAP5 _............................. 97

5.4.3. Experimentos simulados con optimización de parámetros. .99

5.4.3.1. Experimento RIT 136

5.4.3.2. Experimento RIT $139 \ldots \ldots$

5.4.3.3. Consideraciones finales de la optimización _............ 110

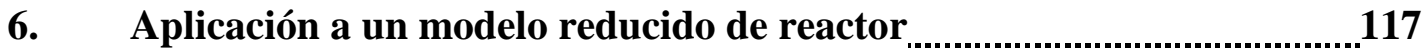

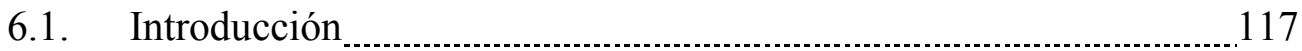

6.2. Planta de referencia ....................................................... 118

6.3. Modelo reducido de reactor PWR 120

6.4. Ajuste y optimización de parámetros

6.4.1. Ajuste de parámetros en estado estacionario _.................... 130

6.4.2. Búsqueda y optimización de parámetros ante transitorios de inserción de reactividad ................................ 132

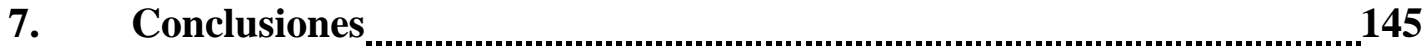

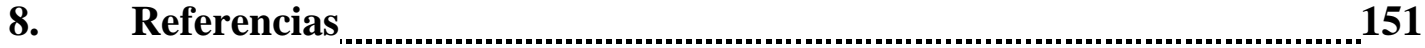

APÉNDICE 1: Fichero de entrada del Edward's pipe para RELAP5.

APÉNDICE 2: Fichero de entrada del RIT 136 para RELAP5.

APÉNDICE 3: Fichero de entrada del PWR de dos lazos para RELAP5. 


\section{LISTADO DE FIGURAS}

Figura 1.1: Esquema de la instalación BETHSY para RELAP5 3

Figura 2.1: Mapa de regímenes para una tubería vertical de RELAP5 12

Figura 2.2: Esquema de la metodología CSAU

Figura 3.1: Ciclo evolutivo de un AGEE 29

Figura 3.2: Cruce por un punto 31

Figura 3.3: Proceso de mutación "Flip mutator"

Figura 3.4: Criterio de terminación _................................... 34

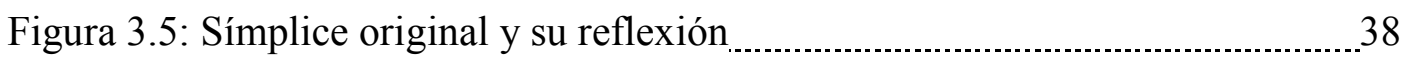

Figura 3.6: Esquema del método MDS _ 39

Figura 3.7: Símplice original con el reflejado y su expansión _.............................. 41

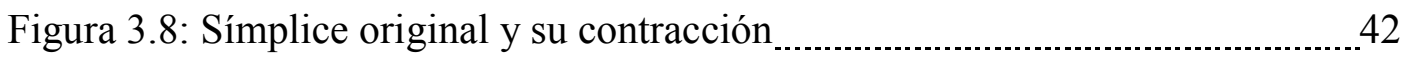

Figura 3.9: Nuevas direcciones de búsqueda en iteraciones consecutivas _............. 44

Figura 3.10: Efecto de nuevos pasos de búsqueda en iteraciones consecutivas .......44

Figura 4.1: Condición inicial del problema de difusión del calor $\quad 50$

Figura 4.2: Función $\mathrm{g}(\mathrm{x})$ utilizada para el cálculo del desarrollo en serie de Fourier de las condiciones iniciales _.......................... 52

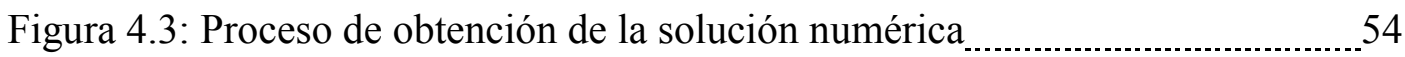

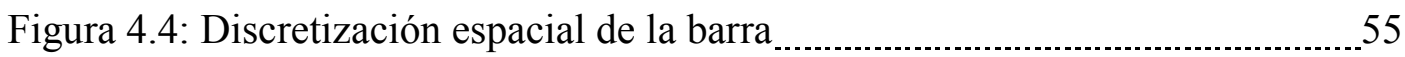

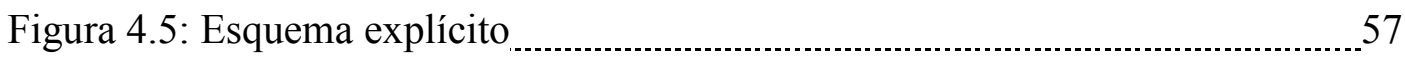

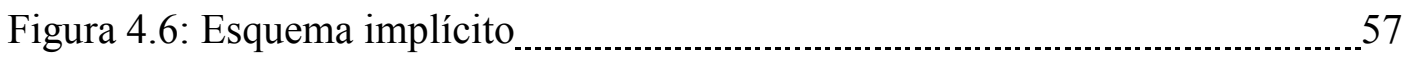

Figura 4.7: Ejemplo de disposición de detectores y discretización numérica _....... 59

Figura 4.8: Condición inicial para el problema de convección-difusión _............ 68

Figura 4.9: Perfiles de temperatura calculados con respecto al de referencia _........ 76

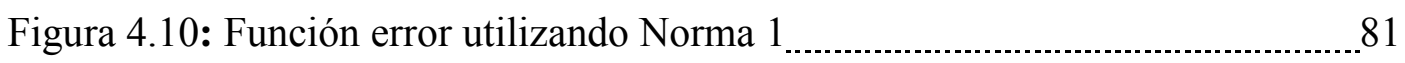

Figura 5.1: Nodalización del Edward's pipe para RELAP5

Figura 5.2: Evolución de la temperatura en el problema Edward's pipe ...................93

Figura 5.3: Esquema simplificado de la Instalación RIT _................................... 95

Figura 5.4: Localización de los termopares en la zona calentada ...........................96

Figura 5.5: Nodalización de la instalación RIT para RELAP5

Figura 5.6: Comparación entre las medidas experimentales y el caso base para RIT136.............................................................

Figura 5.7: Perfil axial de temperatura. Optimización 1 parámetro RIT136 ............ 101

Figura 5.8: Perfil axial de temperatura. Optimización 2 parámetros RIT136_........ 103

Figura 5.9: Perfil axial de temperatura. Optimización 3 parámetros RIT136 ........... 105

Figura 5.10: Comparación entre las medidas experimentales y el caso base para RIT139 
Figura 5.11: Perfil axial de temperatura. Optimización 1 parámetro RIT139 107

Figura 5.12: Perfil axial de temperatura. Optimización 2 parámetros RIT139 _....... 108

Figura 5.13: Perfil axial de temperatura. Optimización 3 parámetros RIT139 $\ldots 110$

Figura 5.14: Curva de ebullición del RELAP5 $\ldots . \ldots 11$

Figura 6.1: Esquema del modelo reducido de reactor PWR de dos lazos _.............. 120

Figura 6.2: Esquema simplificado de la vasija y combustible $\ldots$

Figura 6.3: Evolución de la potencia con RELAP5 _......................................... 131

Figura 6.4: Comparación de las evoluciones de la potencia tomando como referencia la potencia

Figura 6.5: Comparación de las evoluciones de la temperatura del combustible tomando como referencia la potencia

Figura 6.6: Comparación de las evoluciones de la potencia tomando como referencia la temperatura del combustible.

Figura 6.7: Comparación de las evoluciones de la temperatura del combustible tomando como referencia la temperatura del combustible

Figura 6.8: Comparación de las evoluciones de la potencia tomando la potencia

y la temperatura del combustible como referencias 138

Figura 6.9: Comparación de las evoluciones de la temperatura del combustible tomando la potencia y la temperatura del combustible como referencias

Figura 6.10: Comparación de las evoluciones de la potencia para los dos transitorios

Figura 6.11: Comparación de las evoluciones de la temperatura del combustible para los dos transitorios

Figura 6.12: Comparación de las evoluciones de la potencia para los dos transitorios con los parámetros óptimos de la inserción negativa

Figura 6.13: Evolución de la temperatura del combustible para los dos transitorios con los parámetros óptimos de la inserción negativa

\section{LISTADO DE TABLAS}

Tabla 2.1: Características neutrónicas e hidrodinámicas de algunos códigos termohidráulicos

Tabla 2.2: Métodos numéricos de algunos códigos termohidráulicos

Tabla 4.1: Características del MDS para 1 parámetro. 60

Tabla 4.2: Análisis de la dependencia del número de detectores _..........................61

Tabla 4.3: Análisis de la dependencia del paso de integración espacial .................... 62

Tabla 4.4: Análisis del efecto del tiempo de muestreo _.............................................. 63

Tabla 4.5: Análisis en función del paso temporal de integración .............................. 64 
Tabla 4.6: Características del AGEE para 1 parámetro

Tabla 4.7: Análisis de sensibilidad para el AGEE de 1 parámetro...........................66

Tabla 4.8: Resultados a 200 generaciones _ 67

Tabla 4.9: Características del MDS para 2 parámetros _................................... 74

Tabla 4.10: Análisis de la dependencia del número de nodos _....................... 75

Tabla 4.11: Análisis de la dependencia del paso temporal de integración (1) _....... 77

Tabla 4.12: Análisis de la dependencia del paso de temporal de integración (2)..... 78

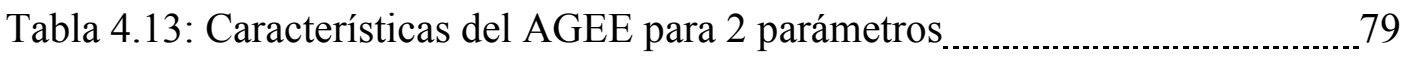

Tabla 4.14: Análisis de la dependencia del numero de nodos _............................. 80

Tabla 5.1: Matriz de valoración del RELAP5/Mod3.0 para LBLOCAs en PWR 86

Tabla 5.2: Matriz de valoración del RELAP5/Mod3.0 para SBLOCAs en PWR 87

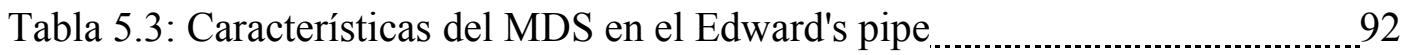

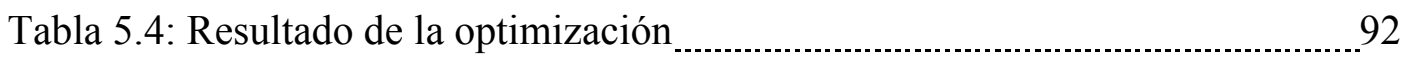

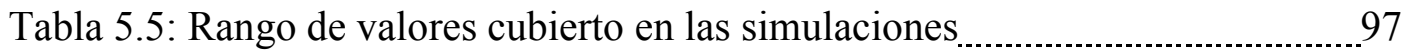

Tabla 5.6: Valores de la optimización del RIT136 para 1 parámetro _.................... 100

Tabla 5.7: Valores de la optimización del RIT136 para 2 parámetros _.................. 102

Tabla 5.8: Valores de la optimización del RIT136 para 3 parámetros _................... 104

Tabla 5.9: Valores de la optimización del RIT139 para 1 parámetro $\ldots$

Tabla 5.10: Valores de la optimización del RIT139 para 2 parámetros _ 108

Tabla 5.11: Valores de la optimización del RIT139 para 3 parámetros $\quad 109$

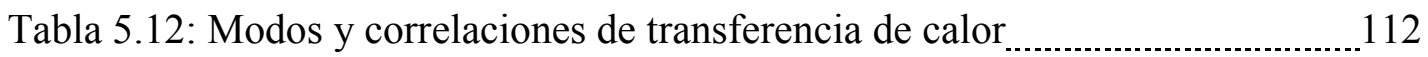

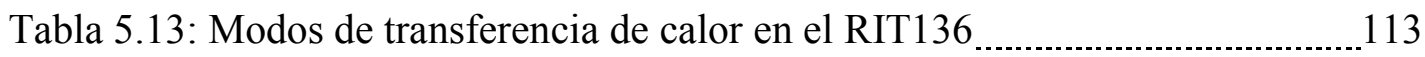

Tabla 5.14: Modos de transferencia de calor en el RIT139

Tabla 6.1: Parámetros de las ecuaciones de la cinética puntual _............................

Tabla 6.2: Valores de las variables del modelo reducido en el estacionario ............ 132

Tabla 6.3: Valores iniciales para los parámetros del modelo reducido _.................... 133

Tabla 6.4: Valores de la optimización tomando la potencia como referencia _......... 134

Tabla 6.5: Valores de la optimización tomando la temperatura del combustible como referencia

Tabla 6.6: Valores de la optimización tomando dos referencias ...............................137

Tabla 6.7: Errores calculados en cada optimización _........................................ 139

Tabla 6.8: Valores de la optimización de la inserción negativa de reactividad

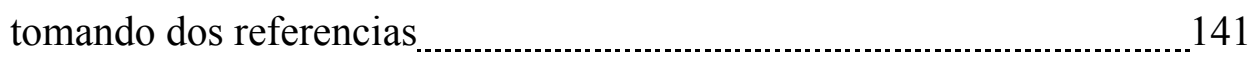

Tabla 6.9: Errores obtenidos en los distintos cálculos considerados ........................ 143 



\section{Capítulo 1}

\section{Introducción}

\subsection{Antecedentes}

La evaluación del comportamiento de las plantas nucleares cuando se produce un escenario transitorio es un tema principal de investigación dentro del campo de la seguridad nuclear desde que se comenzó la explotación de la energía nuclear para la generación de energía eléctrica. Sin embargo, al igual como ocurre en muchos otros campos de la ingeniería, la comprobación experimental del comportamiento de las plantas nucleares no es viable en la mayoría de los casos, por lo que se hace necesaria la simulación de las secuencias accidentales de las que se puedan extrapolar los resultados a las instalaciones reales.

En los últimos años, el desarrollo de las capacidades de cálculo y de los métodos numéricos que se utilizan para calcular los valores de las variables que rigen el comportamiento de la planta, ha incentivado el uso de las simulaciones como una herramienta indispensable en los análisis de la seguridad de la planta.

En este sentido, en agosto 1988 la Nuclear Regulatory Commision (NRC) estableció una norma en la que se recogen los criterios de aceptación del comportamiento del sistema de refrigeración de emergencia del reactor (ECCS) en la cual se establece la necesidad de realizar análisis de secuencias accidentales mediante códigos termohidráulicos de estimación óptima que puedan proporcionar estimaciones más realistas de los márgenes de seguridad de las plantas, siempre que el usuario cuantifique la incertidumbre de las estimaciones y la incluya en la comparación de los resultados calculados con los establecidos en los límites de aceptación. 
En efecto, todas las simulaciones llevan asociadas una cierta incertidumbre puesto que los códigos de simulación utilizan métodos numéricos que proporcionan un valor aproximado de una determinada variable (INEL1995a). Además, existen aspectos de determinados fenómenos físicos que no están completamente implementados en los códigos o se realizan simplificaciones para abordar los distintos problemas (D'Auria2000). También se utilizan numerosas correlaciones empíricas en las que, en general, no queda completamente especificado el rango de su validez y que a su vez se obtienen como un promedio de datos experimentales dispersos que llevan asociados un determinado error.

Un efecto importante en cuanto a la incertidumbre de una determinada simulación lo constituye el efecto de usuario. En el diseño de un modelo de la planta o instalación sobre la que se realiza un determinado experimento varios grupos de usuarios cualificados llegan normalmente a modelos diferentes. Para un código termohidráulico, el modelo de una planta está formado por el conjunto de datos de entrada que permiten simular su comportamiento y calcular la evolución temporal durante un transitorio de las variables que sean de interés. En general los datos proporcionan información sobre las características físicas y geométricas de los elementos y sistemas analizados como: la vasija del reactor, tuberías, elementos combustibles etc, sobre las características de funcionamiento de equipos de los sistemas de la planta como: bombas, válvulas etc, sobre el comportamiento de los sistemas de control que pueden actuar durante el proceso, sobre el comportamiento del núcleo en cuanto al comportamiento neutónico, y sobre las condiciones anómalas que pueden presentarse en la planta y se deseen simular como roturas de tuberías. En función de cuál sea el alcance del estudio que se desee desarrollar este modelo incluirá más o menos detalles. Como ejemplo la figura 1.1 muestra la representación geométrica del modelo desarrollado por la Universidad Politécnica de Valencia (Carlos1998) para RELAP5/Mod3.2 utilizado en la simulación de un transitorio en el que simulaba la pérdida total del sistema de extracción del calor residual en condiciones de medio lazo para la instalación experimental del Boucle d'Etudes Thermohidrauliques (BETHSY).

La generación del modelo de planta empieza con la discretización del sistema real en una red de nodos conectados entre sí, mediante los que se reproduce el camino que sigue el refrigerante dentro del sistema. El código calcula la evolución temporal de las variables tremohidráulicas como: temperatura, caudal, presión etc, en cada uno de los elementos de esta red. Los criterios para la discretización vienen dados por la experiencia lograda en la propia validación del código y por la acumulada en su uso. Una vez construido el modelo se le especifican las condiciones iniciales de contorno para definir el estado de operación de la planta. 


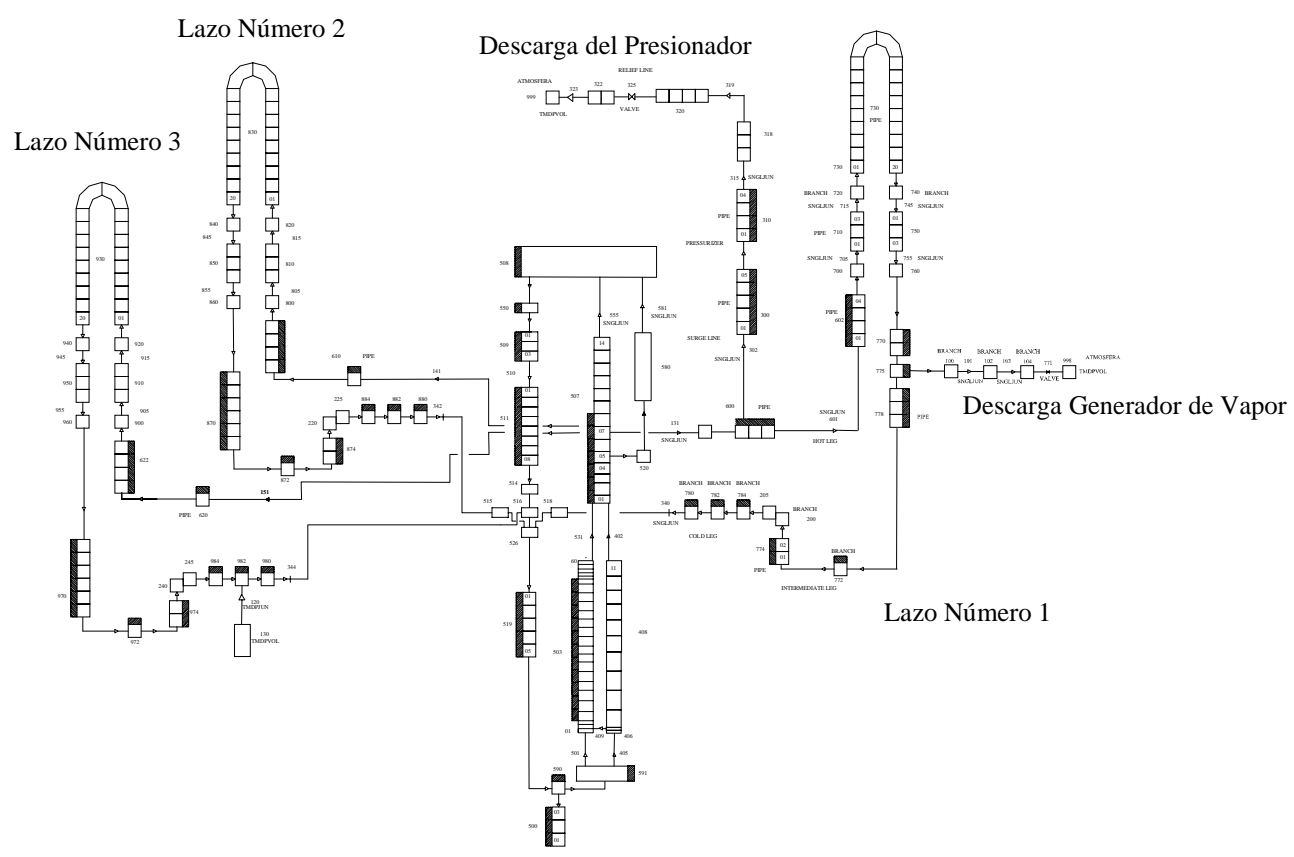

Figura 1.1: Esquema de la instalación BETHSY para RELAP5

Los datos para el diseño de los modelos de planta se obtienen de planos de los distintos componentes, diagramas de proceso, especificaciones de funcionamiento, hojas de datos de instrumentos etc. Sin embargo, la elección de algunas de las opciones que ofrece el código al desarrollar la nodalización que representa la instalación de referencia es en algunos casos arbitraria, debido a la necesidad de interpretar la información disponible, que generalmente no es suficientemente exhaustiva para cubrir todos los datos que se requieren en el fichero de entrada del código, o bien porque los valores iniciales de muchas de las variables e incluso de las condiciones de contorno del modelo son en muchas ocasiones desconocidos o aproximados, y el usuario debe decidir sobre el valor a adoptar.

Todos estos factores hacen necesario un proceso de validación del modelo desarrollado que implica la comprobación de la validez del código que se está utilizando: de sus modelos constitutivos, ecuaciones, métodos numéricos de resolución etc; la comprobación de la validez de las pautas de modelación seguidas en función del escenario y las lecciones aprendidas en experimentos; la determinación de la validez de los datos utilizados, ya sean de diseño, de operación etc; y por último, deben de cuantificarse, y acotarse en la medida de lo posible, las incertidumbres introducidas en todo el proceso de simulación para garantizar que los resultados obtenidos son representativos de la instalación real. 
La cuantificación y acotación de dicha incertidumbre puede realizarse mediante varias metodologías que se encuentran desarrolladas y validadas. Entre estas metodologías cabe señalar la Code Scaling, Aplicability and Uncertainty (CSAU) (Boyack1990; Catton1990; William1986; Wilson1990; Wulf1990) y la Uncertainty Methodology Based on Accuracy Extrapolation (UMAE) (D'Auria1995).

Tanto la metodología CSAU como la UMAE utilizan la comparación de los resultados de las simulaciones con los datos obtenidos de la realización de experimentos en las instalaciones. En el procedimiento de ambas metodologías es necesaria la realización de diferentes análisis de sensibilidad, los cuales dependen en gran medida del usuario que realiza el análisis.

En definitiva, en la realización de dicha comparación se pueden distinguir dos puntos de introducción de errores en función de la fase de simulación:

- Diseño

- Ejecución

Dentro de la primera se pueden asociar a su vez fuentes de error con:

- Modelos termohidráulicos.

- Correlaciones utilizadas por los modelos termohidráulicos y datos.

- Nodalización que, a su vez, se puede subdividir en:

- Componentes del modelo.

- Discretización y acople de componentes.

- Condiciones de contorno.

Dentro de la segunda fase, se pueden diferenciar:

- Métodos numéricos de resolución.

- Condiciones iniciales.

Por otro lado, se tienen dos tipos fundamentales de causas que inducen a la aparición de errores asociados con las fuentes anteriores:

- Limitaciones en modelos y datos.

- Efectos de usuario. 
Ambas causas pueden introducir errores en las fases de diseño y ejecución de una simulación. La diferencia fundamental entre ambas estriba en que las limitaciones en modelos y datos son inherentes al código, por tanto no se pueden eliminar, aunque si pueden cuantificarse, mientras que los segundos son imputables al usuario y por tanto se pueden acotar, cuantificar, y en determinados casos eliminar.

En concreto, la segunda causa de error influye en la fase de diseño a través de los parámetros fijados para la determinación y utilización de correlaciones, componentes, discretización y condiciones de contorno para la nodalización, mientras que en la fase de ejecución influyen fundamentalmente mediante la elección de las condiciones iniciales, y en menor medida a través de la numérica, ya que dicha posibilidad en la mayoría de los códigos no está accesible al usuario.

Por consiguiente, una mejora en cualquiera de ambas metodologías de cálculo y acotación de incertidumbres en la utilización de los códigos termohidráulicos es la posibilidad de realizar los análisis de sensibilidad de forma automática, mediante la búsqueda de las fuentes de error en cualquiera de los elementos discutidos previamente y en la minimización del error cometido a partir de la optimización de los parámetros significativos en cada caso, tratando de eliminar de este modo los efectos de usuario y acotando las incertidumbres que son inherentes a las limitaciones del código utilizado.

Para ello, es necesario que los valores utilizados en los análisis de sensibilidad no se elijan al azar sino que sigan una estrategia de búsqueda y optimización hacia aquellos valores que proporcionen los resultados más ajustados a la referencia. En este sentido existen varias técnicas de búsqueda y optimización respaldadas por una base matemática (Díaz1996; Kelley1999; Torzcon1989; Goldberg1989; Michalewicz1996) y por su experiencia en las aplicaciones en diferentes campos de la ingeniería que son susceptibles de ser aplicadas, de las cuales debe elegirse la más adecuada en función del problema a resolver.

En concreto dentro del campo nuclear, se han realizado distintos estudios con éxito que hacen uso de metodologías de optimización como, por ejemplo, en problemas de optimización de especificaciones técnicas y mantenimiento (Martorell2000c; Martorell2000d) o en el estudio de las posibles configuraciones del núcleo (Abreu1999; François1999), cuya aplicación a la búsqueda y optimización de parámetros termohidráulicos podría ayudar a lograr dicho propósito. 


\subsection{Objetivo}

El objetivo de la presente tesis se centra en estudiar la viabilidad de la aplicación de dichas técnicas al problema de búsqueda y optimización de parámetros de códigos termohidráulicos con el fin de minimizar el efecto de usuario sobre las incertidumbres en los resultados de las simulaciones.

En concreto, se pretende desarrollar y comprobar la aplicabilidad de un método sistemático y automático de búsqueda de parámetros termohidráulicos que resulten desconocidos o inciertos al realizar una simulación, de manera que realice una cuantificación del error cometido en la misma y permita su optimización hacia aquellos valores que lo minimicen. Para ello se utilizarán dos técnicas diferentes de búsqueda, una basada en algoritmos genéticos y otra en un método de búsqueda directa.

\subsection{Organización del trabajo}

El trabajo desarrollado en esta tesis se ha organizado en ocho capítulos, en los que se incluyen este primer capítulo dedicado a introducir y justificar el trabajo presentado posteriormente, y el octavo de referencias utilizadas en la realización de la misma.

En el segundo capítulo se presenta una visión general de los códigos termohidráulicos y de una de las metodologías de cálculo de incertidumbres de simulaciones termohidráulicas que más se utilizan dentro del campo nuclear. Este capítulo presenta el marco en el cual se sitúa la presente tesis y evidencia la necesidad de la misma.

En el tercer capítulo se presenta el planteamiento general del problema que supone la simulación de un escenario transitorio en una determinada instalación. Asimismo, se propone un método sistemático para la mitigación de los errores introducidos a lo largo del proceso de diseño y ejecución de la simulación de escenarios, el cual utiliza una de las técnicas de búsqueda automática que también son detalladas en este mismo capítulo, siendo el algoritmo genético de estado estacionario (AGEE) y el algoritmo de búsqueda multidireccional (MDS).

En el cuarto capítulo se presentan los resultados de la aplicación de la citada metodología para la resolución de problemas sencillos de búsqueda y optimización de parámetros de modelos que simulan procesos termohidráulicos básicos que normalmente se incluyen en los códigos termohidráulicos. El propósito de este capítulo es doble. Por un lado, mediante problemas sencillos se demuestra la viabilidad de la metodología para acotar las incertidumbres asociadas con la resolución numérica, cuyas conclusiones son extrapoladas a situaciones más complejas que se encuentran 
normalmente presentes en las simulaciones mediante códigos termohidráulicos de estimación óptima. En segundo lugar, pero no menos importante, se lleva a cabo una intercomparación de ambas técnicas de búsqueda de manera que es posible identificar la más eficiente para la resolución de este tipo de problemas. Las conclusiones extraídas de estos casos sencillos son igualmente extrapolables a la utilización de modelos complejos, dado que el comportamiento esperado de los mismos es similar, por serlo el tipo de modelos utilizados, lo que permite concluir la técnica de búsqueda más apropiada, la cual será utilizada para resolver este tipo de problemas en las aplicaciones de la metodología dentro de los siguientes capítulos.

El quinto capítulo aborda la aplicación de la metodología a problemas de simulación en los que se utiliza un código de estimación óptima. El propósito de este capítulo es demostrar la viabilidad de la metodología propuesta para cuantificar y acotar las incertidumbres asociadas con la utilización de modelos termohidráulicos más complejos que los del capítulo precedente. En concreto, el código termohidráulico elegido ha sido el RELAP5/Mod3.2. Los casos de aplicación se han considerado, por un lado el "Edward's pipe" que es uno de los transitorios suministrados junto con RELAP para comprobar que su instalación se ha llevado a cabo con éxito. Por otra parte, se ha aplicado la metodología para el análisis de dos experimentos de efectos separados realizados en la instalación RIT (Royal Institute of Technology) con la finalidad de realizar una intercomparación entre los datos experimentales y los cálculos proporcionados por el RELAP5. En ninguno de estos ejemplos ha sido posible la consideración de una planta completa, sino que los modelos utilizados son más simplificados debido a que el tiempo invertido en la simulación por el RELAP hace ineficiente el uso de esta metodología.

En el sexto capítulo se presenta el desarrollo de un modelo reducido de reactor del tipo PWR sobre el que se aplica la metodología de búsqueda y optimización de parámetros para cuantificar y acotar las incertidumbres en los parámetros del modelo. Los modelos simplificados de reactores se utilizan para realizar un estudio cualitativo del comportamiento de las variables representativas de la planta ante transitorios normales de operación, puesto que en el caso de intentar reproducir posibles accidentes es necesaria la simulación de la instalación mediante modelos más detallados. En este capítulo se propone el desarrollo de un modelo reducido de PWR de dos lazos demostrando la utilidad de la metodología de búsqueda y optimización para la determinación de las constantes efectivas del modelo.

En el séptimo capítulo se presentan las conclusiones más relevantes extraídas de la tesis que cubren los objetivos propuestos en esta introducción junto con las aportaciones realizadas en la misma. 
Capítulo 1 


\section{Capítulo 2}

\section{Fundamentos}

\subsection{Códigos termohidráulicos}

Existen numerosos campos de la ciencia y de la ingeniería en los que interesa conocer las condiciones y comportamientos de los fluidos, como la ingeniería aeronáutica, la ingeniería nuclear, las previsiones meteorológicas etc., en los que la experimentación resulta en muchos casos difícil o inviable. En todos estos campos, desde mediados de los años cincuenta apareció, con la mejora en la velocidad de cálculo de los ordenadores, la posibilidad de realizar simulaciones como complemento a la experimentación. Además de resultar más convenientes desde el punto de vista económico, ofrecen otras ventajas como la posibilidad de ampliar el rango de validez de los resultados teóricos mediante la comprobación de los mismos en condiciones inviables experimentalmente, poder reproducir varias veces un mismo experimento, incluso cambiando las condiciones de contorno o geometría sin que esto suponga unos costes excesivos del estudio, y proporciona información más detallada de los fenómenos que se están simulando.

La mejora en la eficiencia de los ordenadores ha llevado consigo la mejora en los esquemas de cálculo numérico que se utilizan en las simulaciones para obtener una solución aproximada al problema que se esté estudiando. El proceso total para la determinación de información sobre la evolución de las variables en problemas que impliquen movimiento de fluidos y/o transmisión de calor, cambio de fase etc, consiste básicamente en la resolución de un conjunto de ecuaciones de estado. Estas ecuaciones son ecuaciones en derivadas parciales difíciles de resolver. Por ello, se utilizan métodos aproximados para su resolución que las reemplazan por un sistema de ecuaciones algebraico de más fácil resolución. 
En el campo nuclear la aplicación de este tipo de análisis para simular los diferentes fenómenos físicos que se pueden producir en una central empezó a mediados de los sesenta, ante la necesidad de simular el comportamiento termohidráulico del sistema de refrigeración de emergencia del núcleo de un reactor de agua ligera ante un accidente de pérdida de refrigerante. A partir de ese momento y hasta la actualidad, se han ampliado los escenarios transitorios a estudiar, lo que ha llevado a la mejora y al aumento en la sofisticación de los códigos de simulación para que sean capaces de reproducir cada uno de los procesos físicos que se producen en un determinado transitorio.

Los códigos de cálculo para la modelización de procesos termohidráulicos son herramientas utilizadas en las plantas nucleares para predecir su comportamiento en diferentes situaciones operativas, que pueden incluir un amplio conjunto de situaciones accidentales. Estas técnicas de simulación se aplican en diferentes ámbitos dentro de la gestión técnica de la explotación de las centrales, entre los cuales se puede citar:

- El análisis del núcleo y de canal de refrigeración del combustible, que se utiliza en el diseño de recargas y en el seguimiento y gestión del núcleo del reactor.

- Análisis de transitorios para determinar la evolución dinámica de la planta como consecuencia de fallos de equipos y de operación, lo cual permite establecer unos límites en las condiciones normales de operación para que no se superen en ningún momento los límites de seguridad.

- Análisis de accidentes, como los que presentan pérdida de refrigerante en los que se producen fenómenos físicos complejos que requieren capacidades especiales en la modelización de los procesos fluidodinámicos y de transmisión de calor.

Además de estas utilidades, con los códigos termohidráulicos se realizan estudios de revisión y mejora de los márgenes de seguridad de las plantas que complementan los estudios del Análisis Probabilista de Seguridad (APS) de las centrales, ayudando a la determinación de secuencias, criterios de éxito y tiempos disponibles de acciones del operador que se encuentran postulados en el mismo.

Los códigos termohidráulicos para la simulación del comportamiento de una planta nuclear disponen de un amplio conjunto de modelos para simular cada uno de los fenómenos físicos que pueden tener lugar en la misma en función de las condiciones de operación que se quieran simular. Así, cualquier código que pretenda simular el funcionamiento de una planta debe disponer de: Un modelo neutrónico y un modelo de transmisión de calor, para dar cuenta de la producción y transporte del calor generado en el núcleo del reactor, un modelo hidrodinámico, modelos de componentes y sistemas 
de control que describan, de un modo fiable, la fenomenología de los fluidos en dos fases dentro de un sistema nuclear.

Básicamente, los modelos neutrónicos disponibles en estos códigos pueden ser: de cinética puntual, de cinética 1D y de cinética 3D, dependiendo de la geometría utilizada para resolver la ecuación de la difusión. En la tabla 2.1 se presentan algunos códigos termohidráulicos con algunas de sus características neutrónicas.

\begin{tabular}{|c|c|c|c|}
\hline Código & Reactor & Neutrónica & Termohidráulica \\
\hline \multirow{3}{*}{ RELAP5 } & \multirow{3}{*}{ PWR } & \multirow{3}{*}{ Cinética puntual, 3D } & Masa: líquido, vapor \\
\hline & & & Energía: líquido, vapor \\
\hline & & & Momento: líquido, vapor \\
\hline \multirow{3}{*}{ TRAC-B } & \multirow{3}{*}{ BWR } & \multirow{3}{*}{ Cinética puntual, 1D, 3D } & Masa: líquido, vapor \\
\hline & & & Energía: líquido, vapor \\
\hline & & & Momento: líquido, vapor \\
\hline \multirow{3}{*}{ TRAC-P } & \multirow{3}{*}{ PWR } & \multirow{3}{*}{ Cinética puntual, 1D, 3D } & Masa: líquido, vapor \\
\hline & & & Energía: líquido, vapor \\
\hline & & & Momento: líquido, vapor \\
\hline \multirow{3}{*}{ CATHARE } & \multirow{3}{*}{ PWR } & \multirow{3}{*}{ Cinética puntual } & Masa: líquido, vapor \\
\hline & & & Energía: líquido, vapor \\
\hline & & & Momento: líquido, vapor \\
\hline \multirow{3}{*}{ ATHLET } & \multirow{3}{*}{ PWR, BWR } & \multirow{3}{*}{ Cinética puntual, 1D, 3D } & Masa: líquido, vapor \\
\hline & & & Energía: Líquido, vapor \\
\hline & & & Momento: mezcla \\
\hline \multirow{3}{*}{ RETRAN } & \multirow{3}{*}{ PWR, BWR } & \multirow{3}{*}{ Cinética puntual, 1D, 3D } & Masa: mezcla \\
\hline & & & Energía: mezcla \\
\hline & & & Momento: mezcla \\
\hline
\end{tabular}

Tabla 2.1: Características neutrónicas e hidrodinámicas de algunos códigos termohidráulicos.

En cuanto a los modelos hidrodinámicos, éstos se pueden diferenciar en función de las fases que considera el modelo para simular los fluidos. Básicamente, el sistema de ecuaciones a resolver está formado por la ecuación de conservación de la masa, la de la energía y la del momento, por lo que para los modelos más sencillos se reduce a la resolución de un sistema de tres ecuaciones para la mezcla formada por el agua y el vapor. Si en el modelo se contempla el comportamiento bifásico de los fluidos, las ecuaciones a resolver son las anteriormente citadas para cada una de las fases, incrementándose por tanto el número de ecuaciones hasta un máximo de seis, que puede ser menor puesto que algunos modelos consideran en alguna ecuación sólo el comportamiento de la mezcla y por tanto puede reducirse el número de ecuaciones en el sistema. En la tabla 2.1 se presentan las ecuaciones que utilizan los modelos hidrodinámicos de algunos de los códigos de estimación óptima. 
En la descripción completa del comportamiento bifásico de los fluidos no basta con resolver el sistema de ecuaciones planteado anteriormente, sino que existen parámetros como la tasa de generación de vapor, la fricción y la transmisión de calor entre fases, etc, que dependen de la distribución del líquido y del vapor dentro de un volumen de control. A las diferentes distribuciones que se pueden simular se les denomina regímenes de flujo, y dependen principalmente de la cantidad de vapor, de las velocidades de ambas fases y de la geometría del volumen que se considere. La determinación del régimen de flujo se realiza a partir de los mapas de flujo que se han obtenido ajustando correlaciones empíricas para algunas geometrías. Como ejemplo, la figura 2.1 muestra el mapa de regímenes que utiliza el RELAP5 en el caso de una tubería vertical, que depende de la velocidad de la mezcla $\left(\mathrm{v}_{\mathrm{m}}\right)$, de la fracción de huecos $(\alpha)$, y de la diferencia entre la temperatura del vapor $\left(\mathrm{T}_{\mathrm{g}}\right)$ y la temperatura de saturación $\left(\mathrm{T}^{\mathrm{s}}\right)$.

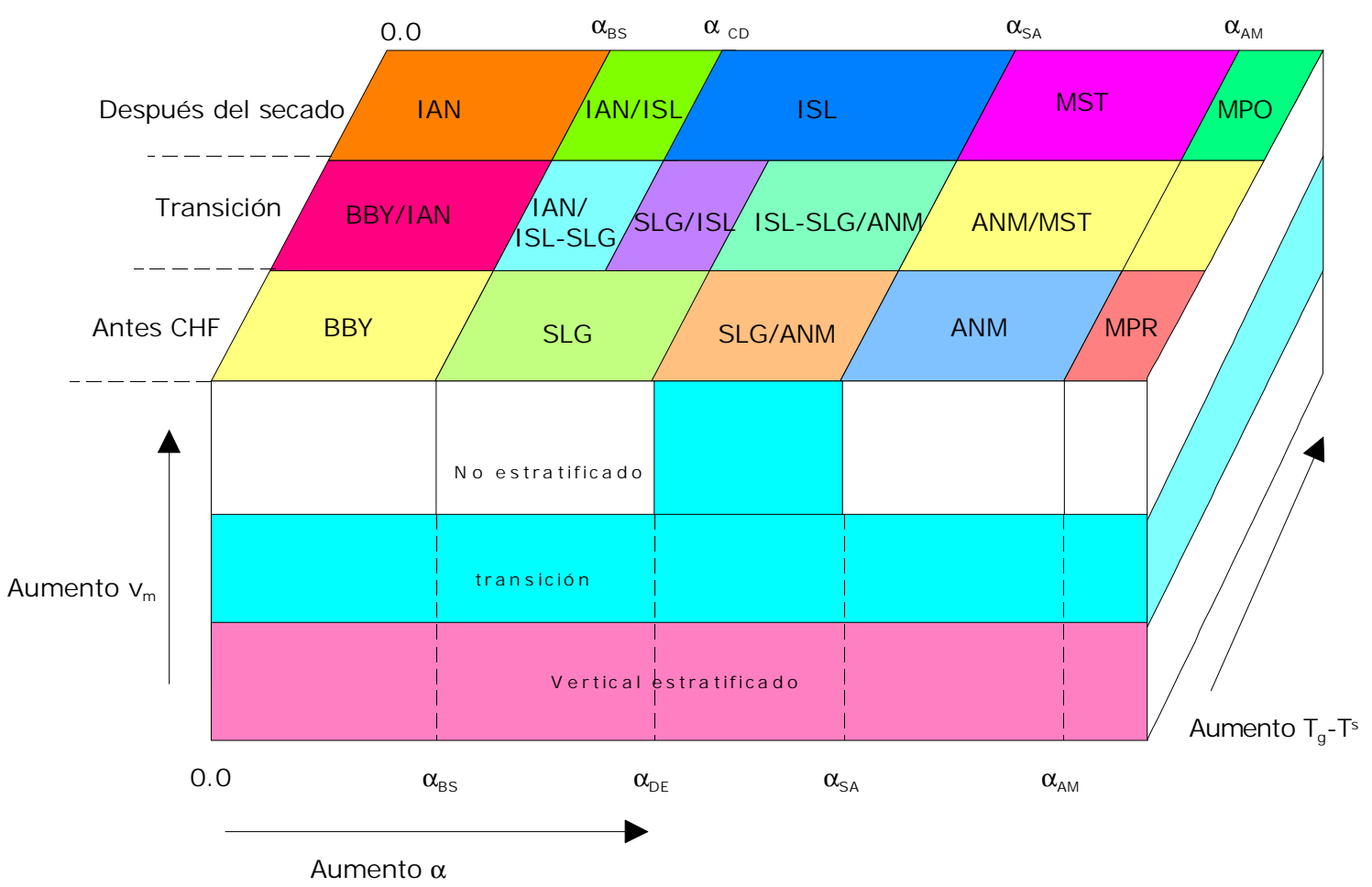

Figura 2.1: Mapa de regímenes para una tubería vertical de RELAP5.

Las siglas de la figura 2.1 hacen referencia a cada uno de los regímenes de fluido que se pueden distinguir. En concreto BBY es el flujo en burbujas; SLG flujo en tramos; ANM flujo anular; IAN flujo anular invertido etc. La información detallada sobre cada uno de los regímenes implementados se encuentra en la referencia (INEL1995d). 
Además, el régimen en el que se encuentra un fluido es necesario para la determinación de la transmisión de calor a las paredes y entre las fases del fluido. En la determinación de los coeficientes de transmisión de calor para determinar la transferencia de calor en el seno de un fluido los códigos termohidráulicos hacen uso de correlaciones empíricas ajustadas en función del régimen de flujo y de las condiciones termodinámicas de cada una de las fases. En el caso de simular la trasmisión de calor entre las paredes y el fluido las correlaciones empíricas que determinan los coeficientes de transferencia de calor dependen del régimen del flujo, de la geometría que se considere, y de la disposición del fluido respecto a la estructura con la que intercambia el calor.

Los códigos tienen implementados modelos especiales para simular el paso de los fluidos a través de válvulas, bombas, generadores etc, de manera que el comportamiento sea el mismo que el que tiene lugar en la planta. Además, los códigos llevan diseñados diversos controles para simular acciones a las que se somete la instalación, como por ejemplo la apertura de una válvula, o la inserción de barras de control.

Para la resolución de las ecuaciones que constituyen los modelos es necesario utilizar aproximaciones numéricas que proporcionan la evolución de las variables de la planta en un tiempo razonable. La mayoría de los códigos termohidráulicos utilizan aproximaciones en diferencias finitas utilizando normalmente esquemas de resolución semiimplícitos o implícitos lo que permite garantizar la estabilidad del código utilizando pasos de integración temporales razonables. En la tabla 2.2 se indican los métodos numéricos utilizados por algunos códigos termohidráulicos.

\begin{tabular}{ll}
\hline Código & Método numérico \\
\hline \multirow{2}{*}{ RELAP5 } & Semiimplícito y casi-implícito \\
& Estabilidad: Límite de Courant \\
\hline \multirow{2}{*}{ TRAC-B } & Semiimplícito \\
& Estabilidad: Límite superior al límite de Courant \\
\hline \multirow{2}{*}{ TRAC-P } & Método semiimplícito de tres pasos \\
& Estabilidad: Límite superior al límite de Courant \\
\hline \multirow{2}{*}{ CATHARE } & Método implícito \\
\hline \multirow{2}{*}{ ATHLET } & Estabilidad: incondicionalmente estable \\
\hline \multirow{2}{*}{ RETRAN } & Método implícito \\
& Estabilidad: incondicionalmente estable \\
\hline
\end{tabular}

Tabla 2.2: Métodos numéricos de algunos códigos termohidráulicos. 
Cada uno de los códigos termohidráulicos que existen se encuentran en constante revisión y mejora mediante programas como, por ejemplo, el CAMP (Computer Assessment and Maintenance Program) o el ICAP (International Computer Assessment Program) y a través de ejercicios de intercomparación. Uno de los objetivos que se obtienen al someter los códigos a constante revisión es la identificación por parte de la comunidad nuclear de las capacidades y limitaciones del código en la simulación de accidentes para los que no fue diseñado en su momento, puesto que las numerosas correlaciones empíricas implementadas en estos códigos trabajan fuera de rango en algunos de los experimentos que les sirven de base.

Un ejemplo de las mejoras que se introducen en los códigos termohidráulico a través de los programas y ejercicios de intercomparación es el caso del RELAP5. Este código se diseñó en un principio para simular los accidentes de pérdida de refrigerante en plantas PWR mientras la planta se encuentra trabajando en condiciones de plena potencia. Sin embargo, resulta de gran interés disponer de un código termohidráulico que simule cualquier transitorio que pueda producirse en la planta, por lo que la comunidad nuclear ha utilizado el RELAP5 en la simulación de escenarios transitorios con iniciadores y condiciones de trabajo diferentes de los de diseño, entre los que destaca su utilización para la simulación de escenarios transitorios en otros modos de operación de la planta, tales como baja potencia y parada (Hassan1994; Martorell2000a; Martorell2000b).

Las comparación de los resultados obtenidos para un mismo cálculo utilizando diferentes versiones del código (Nilsson1993; Carlos1999) permite verificar las mejoras introducidas en los modelos y correlaciones implementados en el código con el fin de mejorar sus capacidades para simular cualquier escenario susceptible de producirse en una instalación real.

Con el fin de analizar la necesidad de mejorar los modelos físicos implementados en los códigos termohidráulicos se realizó una reunión internacional en Annapolis, Maryland, USA, en noviembre de 1996 organizada por la NRC. Las conclusiones a las que llegaron los expertos se pueden clasificar en cuatro categorías:

- Necesidad de obtener una mayor precisión en las simulaciones de procesos tales como las oscilaciones en reactores BWR, en el enfriamiento del núcleo por circulación natural, disolución de boro etc. Estos análisis hacen necesaria una capacidad de estimación óptima que sólo está implementada parcialmente en los códigos actuales.

- Ampliación de la capacidad de análisis en los reactores existentes. Los códigos deben ser capaces de simular cualquier transitorio especificado en los análisis de seguridad de las plantas. 
- Incorporación de modificaciones basadas en los resultados de programas internacionales contrastados con experimentos.

- Ampliación de la capacidad de análisis en los reactores avanzados. El licenciamiento de los reactores avanzados planteará nuevos problemas que van a requerir de modificaciones o ampliaciones de los códigos.

Todas estas acciones llevarán a una mayor sofisticación en los modelos a utilizar, tales como la introducción de ecuaciones separadas para los diferentes fluidos, mejora en la definición de los regímenes de fluido, introducción de geometrías 2D y 3D en las ecuaciones hidrodinámicas, extensión de las condiciones de operación a condiciones de baja presión y bajo fluido, mejora en el tratamiento de los incondensables, tratamiento tridimensional de la neutrónica, etc.

\subsection{Metodología de cálculo de incertidumbres}

De lo dicho en el apartado precedente se deduce que existe un número de limitaciones inherentes a los propios códigos termohidráulicos que en mayor o menor medida afectan a la exactitud con la que éstos son capaces de reproducir las condiciones de funcionamiento real de la instalación. Dichas incertidumbres se encuentran tanto en la fase de diseño del modelo de planta como en el proceso de ejecución de la simulación.

Ante la necesidad de asegurar que los resultados calculados por los códigos termohidráulicos sean representativos del comportamiento real de la planta, se desarrollaron diferentes metodologías para cuantificar la incertidumbre de las simulaciones realizadas.

De todas las metodologías de cálculo de incertidumbres que existen las más utilizadas dentro del ámbito nuclear son la denominada Code Scaling, Applicability and Uncertainty Evaluation Methodology (CSAU) desarrollada por la Nuclear Regulatory Commission y la Uncertainty Methodology Based on Accuracy Extrapolation (UMAE) de la Universidad de Pisa. Aunque algunos de los procedimientos de actuación son ligeramente diferentes, el propósito y la filosofía de ambas son similares por lo que aquí se presenta con detalle sólo la primera de ellas. 


\subsubsection{Metodología CSAU}

Esta metodología de cálculo de incertidumbres de las simulaciones se desarrolló para servir de apoyo a la norma emitida en 1988 por la NRC sobre la revisión de los criterios de aceptación del sistema de refrigeración de emergencia del núcleo. Dicha norma se basa en los resultados obtenidos en simulaciones realizadas mediante códigos de estimación óptima que proporcionan resultados más realistas sobre los márgenes de seguridad de las plantas nucleares, siempre que se cuantifique la incertidumbre de la simulación. Los objetivos que esta metodología pretendía cubrir eran:

- Presentar una base técnica para cuantificar las incertidumbres dentro del contexto de la norma ECC (Emergency Core Cooling System) revisada, en la que se plantea la necesidad de simular el comportamiento de este sistema frente a una situación accidental.

- Proporcionar un método que combine los juicios de los expertos con los análisis cuantitativos para calcular los valores de las incertidumbres.

- Proporcionar una aproximación general y sistemática para:

- definir escenarios,

- evaluar la aplicabilidad del código,

- valorar las capacidades del código

- cuantificar las incertidumbres referentes a: la precisión del código y de los datos experimentales, la escalabilidad del código, el estado de la planta y las condiciones de funcionamiento.

Con el fin de demostrar la validez de la metodología planteada y verificar que todos los objetivos propuestos quedaban cubiertos, se realizó un ejemplo en el que la metodología propuesta se aplicó al estudio de un accidente de pérdida de refrigerante debido a una rotura grande en una planta PWR de cuatro lazos y utilizando para realizar las simulaciones el código termohidráulico TRAC-PF1/MOD1. La descripción de la metodología CSAU junto con los resultados y conclusiones extraídos del caso de aplicación se recogen en las referencias Boyack 1990, Wilson 1990, Wulf 1990, Zuber 1990 y Catton 1990. Aunque se realizó este caso particular de aplicación, la metodología desarrollada es general y aplicable a otros escenarios, plantas y códigos que se pretendan estudiar.

Tomando como punto de partida una planta y un escenario previamente especificados la metodología CSAU se centra sólo en la cuantificación de las incertidumbres introducidas o existentes en la simulación debidas a los procesos y fenómenos físicos importantes que tienen lugar durante el transitorio en estudio y/o en la capacidad del código para escalarlos, evaluando la precisión con la que los calcula. La metodología 
CSAU consta, como se muestra en la figura 2.2, de tres elementos primarios básicos dentro de los cuales se realizan una serie de acciones determinadas.

En el primero de los elementos de la metodología se identifican los requisitos necesarios para la simulación del transitorio y se comparan con las capacidades del código para determinar su aplicabilidad al escenario concreto, además de intentar localizar las potenciales limitaciones del código.

Para llegar a la determinación de la capacidad del código a partir del escenario y planta seleccionados se lleva a cabo una identificación y priorización de los fenómenos que tienen lugar en el escenario transitorio que se realiza mediante las tablas de identificación y priorización de procesos (Process Identification and Ranking Tables, PIRT). Esta ordenación es importante puesto que a lo largo del transitorio se producen muchos fenómenos físicos que no afectan de igual modo al comportamiento de la planta, con lo que se consigue reducir el número de procesos en estudio a aquellos que son más importantes en cuanto a su influencia en el comportamiento general de la planta.

La evaluación de la aplicabilidad del código para simular el transitorio seleccionado se realiza revisando si los modelos implementados en el código son adecuados para calcular los procesos y fenómenos importantes que se han determinado. Las deficiencias y/o limitaciones del código son también evaluadas como efectos posibles que afectan a la incertidumbre en los resultados.

El segundo de los elementos de esta metodología consiste en la valoración de los parámetros y la especificación de los rangos de valores entre los que se encuentran. En este elemento se realizan actividades de valoración de la capacidad del código para el cálculo de los procesos importantes del escenario, comparando los cálculos proporcionados por las simulaciones con los datos experimentales, con el fin de obtener la precisión del código, la capacidad del escalado, y especificar los rangos de variación de los parámetros necesarios para los estudios de sensibilidad. Los datos experimentales con los que se comparan los cálculos provienen de la realización de experimentos de efectos separados (SETs) y de experimentos integrales (ITEs), con los que se confecciona una matriz de valoración que supone una base de datos para la evaluación de la precisión del código en el cálculo de los fenómenos importantes que se producen durante el transitorio.

Con los experimentos de efectos separados se consigue información acerca de la capacidad de los modelos implementados en el código en la simulación de fenómenos físicos concretos. Mientras que la comparación de los cálculos con los datos de los experimentos de efectos integrales sirve para determinar la capacidad de escalado del código. 
El último de los elementos de esta metodología consiste en la realización de análisis de sensibilidad e incertidumbre. La incertidumbre total en un análisis de sensibilidad incluye contribuciones por las limitaciones del código, las imprecisiones en el escalado incluidas en los datos experimentales, y las incertidumbres asociadas al estado de la tecnología de las plantas nucleares en el momento en el que se desea analizar el transitorio. Por lo que el objetivo final de la metodología se alcanza cuando se calculan, se recogen y se combinan cada una de las magnitudes que individualmente contribuyen a la incertidumbre total de la simulación.

Dentro de este tercer elemento se enmarca la determinación del efecto de los parámetros del fichero de entrada del código, en los que se incluyen los parámetros que hacen referencia a condiciones de contorno, a condiciones iniciales, a la nodalización y a parámetros que afecten a los modelos de cálculo del código, los cuales pueden ser fijados por el usuario a través de este fichero. Este es uno de los puntos de la metodología en los que más incidencia tienen los efectos de usuario, puesto que ante la incertidumbre o desconocimiento de algunos de los valores de los parámetros requeridos por el fichero de entrada cada usuario escoge una solución dentro de los rangos de variación de los mismos.

Esta metodología constituye una guía para el cálculo de las incertidumbres de las simulaciones. Sin embargo, como se ha visto, en algunos de los pasos que propone necesita del juicio de expertos sobre el proceso, que generalmente se canaliza a través de la modificación de parámetros del fichero de entrada, lo que es una fuente de errores de usuario. Por ello, el planteamiento y la adopción de un método automático de búsqueda y optimización de parámetros puede mitigar dichos efectos, llegando incluso a acotar las incertidumbres a aquellas que son inherentes a las propias limitaciones del código y de por tanto, sólo tienen solución a partir del estudio y mejora de código, por ejemplo detectando mediante la realización de experimentos tipo SETs o ITEs las posibles deficiencias de los modelos implementados para sustituirlos por otros más adecuados. 


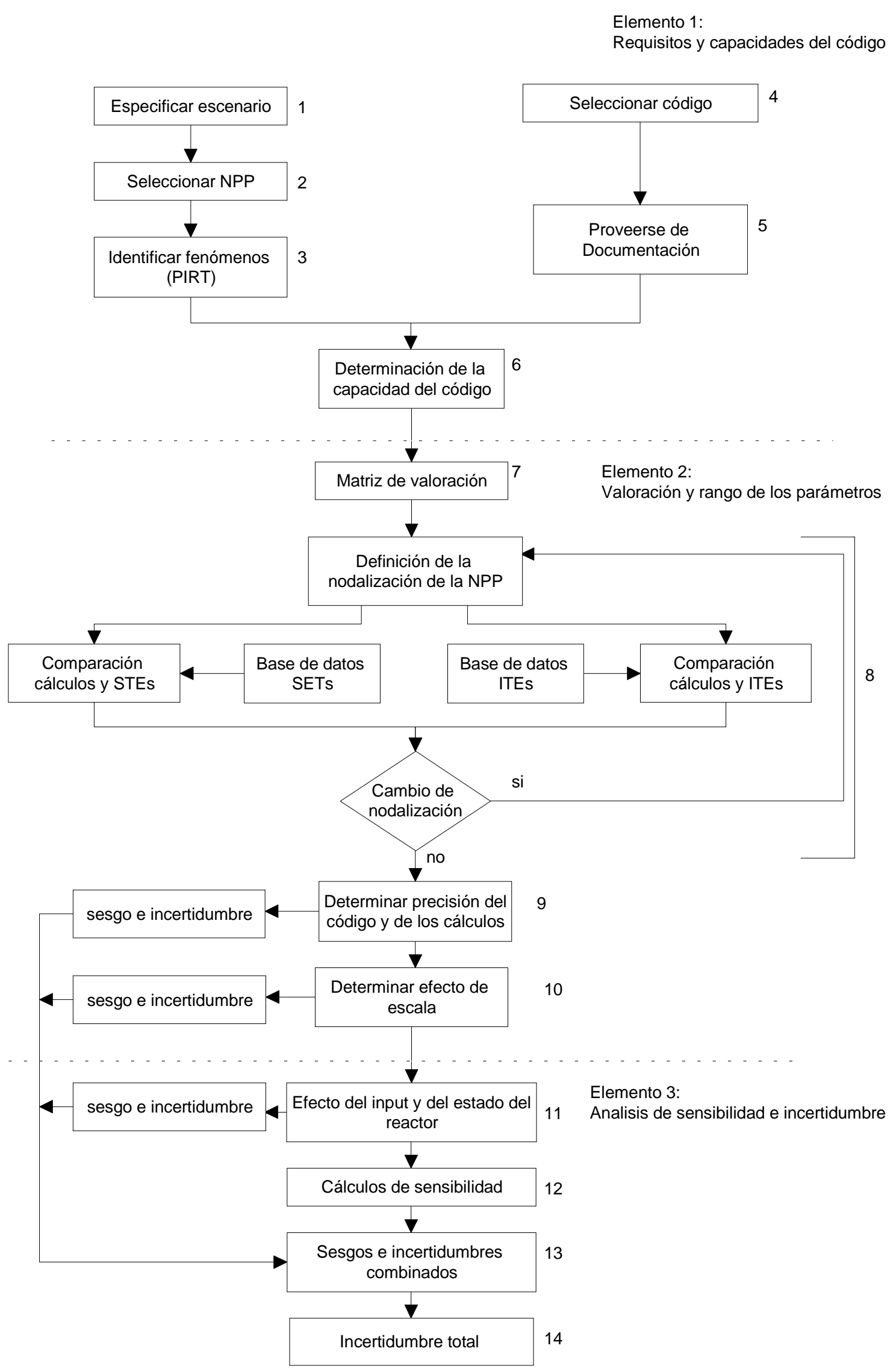

Figura 2.2: Esquema de la metodología CSAU. 
Capítulo 2 


\section{Capítulo 3}

\section{Metodología de búsqueda y optimización de parámetros}

\subsection{Planteamiento y solución del problema}

Hasta el momento se ha mostrado la necesidad de simular diferentes escenarios transitorios para determinar los márgenes de seguridad de las plantas nucleares. Asimismo, se ha visto que toda simulación lleva asociada una cierta incertidumbre debida básicamente a los siguientes factores:

- Los valores de los parámetros que son necesarios para la definición completa del modelo de la planta, introducidos a través del fichero de entrada del código, como por ejemplo condiciones iniciales y de contorno que pueden ser inciertas o desconocidas, la nodalización elegida para definir la instalación y las opciones del fichero de entrada más adecuadas para simular el transitorio.

- Las correlaciones empíricas implementadas en el código termohidráulico para simular los diferentes fenómenos físicos que pueden tener lugar en la instalación durante un determinado transitorio, puesto que en algunos casos puede que las condiciones del transitorio estuvieran incluso fuera del rango de validez de las correlaciones que utiliza, o que los parámetros de los que dependen estuvieran mal ajustados.

- La numérica que utiliza el código para resolver el sistema algebraico resultante de la discretización. 
Por estas razones, se ha visto en el capítulo precedente que se han desarrollado metodologías de cálculo de incertidumbres en simulaciones termohidráulicas con el fin de proporcionar una medida con la que tomar la decisión de aceptar o no los resultados calculados. Sin embargo, aunque estas metodologías proporcionan un modo de actuación adecuado con el fin de conseguir resultados aceptables, en ellas se hace uso en muchos casos del juicio de expertos, lo cual es una fuente de errores de usuario que potencia las incertidumbres anteriomente señaladas e incluso puede añadir otras nuevas.

Este problema se puede minimizar con el planteamiento de una estrategia sistemática y automatizada de toma de decisiones que busque los parámetros más adecuados y determine los valores óptimos para que los cálculos proporcionados por el código sean lo más ajustados posible a una referencia dada (datos experimentales o analíticos), minimizando en este sentido las incertidumbres e intentando que éstas queden reducidas a aquéllas que sean inherentes a las propias limitaciones del código.

La solución al problema de búsqueda y optimización de parámetros puede plantearse mediante el establecimiento de una función error que cuantifique la diferencia entre los resultados proporcionados por el código y las referencias, la cual se debe utilizar como función objetivo para ser minimizada mediante una técnica de optimización que busque el valor de dichos parámetros que hacen que el error cometido sea mínimo.

Generalmente, los datos que se toman como referencia proceden de experimentos y corresponden a variables de estado como presiones, temperaturas y caudales. Estas medidas pueden representar valores medios o valores locales distribuidos en distintas zonas del reactor. Además, se puede distinguir entre situaciones en las que se dispone de valores de las variables en un instante de tiempo determinado y situaciones en las que se sigue la evolución de las variables durante un intervalo de tiempo.

La diferencia entre los valores proporcionados por los códigos y los datos experimentales se pueden organizar en un vector o en una matriz de errores en función de la distribución espacial y temporal de las medidas. Para cuantificar este error se pueden utilizar distintas normas asociadas a los vectores o a las matrices (Burden1993).

En general, se define una norma \|\| , como una aplicación que va de un espacio vectorial, $\mathrm{V}$, a los reales positivos,

$$
\begin{aligned}
\|\|: \mathrm{V} & \rightarrow \mathrm{R}^{+} \\
\mathbf{x} & \rightarrow\|\mathbf{x}\|
\end{aligned}
$$

que satisface las siguientes propiedades: 
1.- $\|\mathbf{x}\| \geqslant 0 ;\|\mathbf{x}\|=0$ si y sólo si $\mathbf{x}=0$.

2.- $\|\mathbf{x}+\mathbf{y}\| \leqslant\|\mathbf{x}\|+\|\mathbf{y}\|$

3.- $\|\alpha \mathbf{x}\|=|\alpha|\|\mathbf{x}\| ; \alpha \in \mathbb{R}$

donde $\mathbf{x}$ e $\mathbf{y}$ son elementos del espacio vectorial V.

Si $\mathbf{x}=\left(\mathrm{x}_{1}, \ldots, \mathrm{x}_{\mathrm{n}}\right)$ es un vector de $\mathbb{R}^{\mathrm{n}}$, generalmente, se suele utilizar la norma 2 , definida como

$$
\|\mathbf{x}\|_{2}=\sqrt{\sum_{i=1}^{n} x_{i}^{2}}
$$

Otras posibles normas vectoriales son, la norma 1 ,

$$
\|\mathbf{x}\|_{1}=\sum_{i=1}^{n}\left|\mathbf{x}_{\mathrm{i}}\right|
$$

y la norma infinito

$$
\|\mathbf{x}\|_{\infty}=\operatorname{máx}_{\mathrm{i}=1, \ldots, \mathrm{n}}\left\{\mathrm{x}_{\mathrm{i}} \mid\right\} \text {. }
$$

Para las matrices se pueden definir normas matriciales asociadas a las normas vectoriales. Así, si || || es una norma vectorial, dada una matriz A, su norma matricial asociada viene dada por

$$
\|\mathrm{A}\|=\operatorname{máx}_{\mathrm{x} \neq 0}\left\{\frac{\|\mathrm{Ax}\|}{\|\mathrm{x}\|}\right\} \text {. }
$$

Se puede ver (Burden1993) que con esta definición, la norma 1 de una matriz $A \in \mathbb{R}^{\mathrm{n} \times \mathrm{m}}$, se puede calcular como

$$
\|\mathrm{A}\|_{1}=\operatorname{máx}_{1 \leq \mathrm{j} \leq \mathrm{m}}\left\{\sum_{\mathrm{i}=1}^{\mathrm{n}}\left|\mathrm{a}_{\mathrm{ij}}\right|\right\}
$$

siendo $\mathrm{a}_{\mathrm{ij}}$ los elementos de la matriz A.

De igual modo, la norma infinito de A se puede calcular como 


$$
\|\mathrm{A}\|_{\infty}=\operatorname{máx}_{1 \leq \mathrm{i} \leq \mathrm{n}}\left\{\sum_{\mathrm{j}=1}^{\mathrm{m}}\left|\mathrm{a}_{\mathrm{ij}}\right|\right\} .
$$

Otra norma matricial que se suele utilizar, aunque no está subordinada a una norma vectorial (Burden1993), es la norma de Frobenius, definida como

$$
\|\mathrm{A}\|_{\mathrm{F}}=\sum_{\mathrm{i}=1 \mathrm{j}=1}^{\mathrm{n}} \sum_{\mathrm{j}}^{\mathrm{m}}\left|\mathrm{a}_{\mathrm{ij}}\right|
$$

Eligiendo una de estas normas se construye la función objetivo a minimizar que generalmente cuantifica el error relativo entre los valores experimentales y los proporcionados por el código.

Para el estudio de transitorios complicados hay autores que proponen una cuantificación distinta del error (Dauria1994; Ambrosini1990). La duración total del transitorio se divide en ventanas fenomenológicas en las que para cada variable importante se obtiene la diferencia entre el resultado proporcionado por el código y las medidas experimentales, determinando un vector tal como,

$\mathbf{v}=\mathbf{x}_{\text {calc }}-\mathbf{x}_{\text {exp }}$

donde $\mathbf{x}_{\text {calc }}$ es el vector de valores proporcionados por el código y $\mathbf{x}_{\exp }$ es el vector de medidas experimentales.

Posteriormente se calcula la transformada rápida de Fourier de $\mathbf{v}$ y de $\mathbf{x}_{\text {exp }}$, obteniendo los vectores en el dominio de la frecuencia $\mathbf{F}(\mathbf{v}), \mathbf{F}\left(\mathbf{x}_{\mathrm{exp}}\right)$. Con estos vectores se define como función objetivo a minimizar (Dauria1994; Ambrosini1990) la amplitud media adimensional como

$$
\mathbf{A A}=\frac{\|\mathbf{F}(\mathbf{v})\|_{1}}{\left\|\mathbf{F}\left(\mathbf{x}_{\exp }\right)\right\|_{1}} .
$$

Como medida total de la precisión de la simulación se deben combinar las diferentes amplitudes calculadas para las variables importantes de cada ventana fenomenológica en la que se divide el transitorio. De este modo se define la función objetivo a minimizar para el transitorio completo como

$$
(\mathrm{AA})_{\mathrm{tot}}=\sum_{\mathrm{j}=1}^{\mathrm{N}_{\mathrm{var}}}(\mathrm{AA})_{\mathrm{j}}\left(\mathrm{w}_{\mathrm{f}}\right)_{\mathrm{j}},
$$


donde $\mathrm{N}_{\mathrm{var}}$ es el número de variables que se han considerado y $\mathrm{w}_{\mathrm{f}}$ unos factores de ponderación asignados a cada variable y determinados mediante el juicio de los expertos en función de su importancia respecto del fenómeno físico que se ha simulado.

Todas las expresiones planteadas hasta el momento pueden utilizarse como función objetivo en el proceso de búsqueda y optimización de parámetros termohidráulicos utilizando distintas técnicas de optimización. De forma muy simple las técnicas de optimización se pueden dividir en los grupos siguientes:

- Analíticas. Obtienen la información sobre el decrecimiento de la función a partir de su derivada.

- Métodos de búsqueda directa. Se caracterizan por el hecho de que el proceso de toma de decisiones se basa solamente en la información que proporciona el valor de la función; en estos algoritmos no se requiere la información suministrada por la derivada para el cálculo de la dirección de decrecimiento de la función (Kelley1999).

- Métodos heurísticos de búsqueda. Se basan en el valor de la función en diferentes puntos del espacio de búsqueda, sin que se tenga en cuenta ninguna dirección de decrecimiento de la función. El proceso de optimización se realiza a partir de los mejores valores encontrados en el conjunto de puntos analizado.

A priori, ninguna de las técnicas conocidas presenta una ventaja sobre las demás con carácter general, sino que se tiene que analizar cada problema en particular. En este capítulo se presentan dos técnicas distintas de optimización ampliamente utilizadas: Un algoritmo genético de estado estacionario (AGEE) que pertenece a las técnicas heurísticas de optimización y el método de búsqueda multidireccional (MDS) que es un método de búsqueda directa.

\subsection{Algoritmos genéticos}

Los Algoritmos genéticos (AAGG) son técnicas heurísticas de optimización basadas en los conceptos de la selección natural y la genética, los cuales fueron desarrollados por $\mathrm{J}$. Holland en 1.965 sirviendo como punto de partida de todas las implementaciones y aplicaciones conocidas de AAGG (Holland1975). Como consecuencia de su simplicidad, flexibilidad, y facilidad de uso han sido utilizados con éxito en una gran variedad de problemas relacionados con muchas áreas de la ingeniería y las humanidades (Beasely1993a; Beasely1993b). Los fundamentos acerca de cómo trabajan los AAGG, su implementación y su aplicabilidad pueden encontrarse en multitud de artículos y libros 
(Michalewicz1996; Fogel1995). Esta técnica se basa en los mecanismos de selección que utiliza la naturaleza, de acuerdo a los cuales los individuos más aptos de una población son los que sobreviven al adaptarse más fácilmente a los cambios que se introducen en su entorno. Hoy en día se sabe que estos cambios se efectúan en los genes de un individuo (unidad básica de codificación de cada uno de los atributos de un ser vivo), y que sus atributos más deseables se transmiten a sus descendientes cuando éste se reproduce.

Los AAGG parten de una población inicial constituida por una serie de individuos cada uno de los cuales representa una posible solución al problema. A través de un proceso de selección natural y utilizando los denominados operadores genéticos, cruce y mutación, se encuentran los individuos de mayor aptitud. El proceso de selección natural garantiza que los mejores individuos sobreviven en las generaciones siguientes mientras que los operadores genéticos permiten obtener nuevos individuos, así mediante el operador cruce se combinan los genes de dos individuos, denominados padres, para formar nuevos individuos (hijos) los cuales heredan la información genética de sus predecesores. La mutación actúa sobre los individuos modificando su información genética y permitiendo explorar nuevos espacios de búsqueda. Este proceso será descrito de forma más detallada en los apartados siguientes.

El poder de los Algoritmos Genéticos proviene del hecho de que se trata de una técnica robusta que puede tratar con éxito una gran variedad de problemas provenientes de diferentes áreas, incluyendo aquellos en los que otros métodos encuentran dificultades. Si bien no se garantiza que el Algoritmo Genético encuentre la solución óptima del problema, existe evidencia empírica de que se encuentran soluciones de un nivel aceptable, en un tiempo competitivo con el resto de algoritmos de optimización combinatoria.

Los AAGG presentan una serie de ventajas respecto a los métodos tradicionales de optimización, entre las que se pueden mencionar: el hecho de que la presencia de discontinuidades en el espacio de búsqueda tiene un pequeño efecto en el desarrollo del problema de optimización, no se requiere información sobre el gradiente de la superficie de respuesta, o la resistencia a caer en un óptimo local, así como el hecho de poder ser empleados en una gran variedad de problemas de optimización.

No obstante, frente a las ventajas anteriores los algoritmos genéticos presentan una serie de desventajas tales como los problemas para obtener un óptimo global exacto, o el gran número de evaluaciones de la función objetivo requeridos. Esta última característica será uno de los problemas importantes en la búsqueda de parámetros de modelos termohidráulicos como se discutirá más adelante.

La construcción de un AG para un problema determinado implica especificar algunos de sus componentes, entre ellos, los más importantes son: 
1. Una representación para las soluciones potenciales del problema sobre la cual se define el espacio de búsqueda.

2. Un método para generar la población inicial de soluciones potenciales.

3. Una función evaluación que evalúe las soluciones en términos de su adaptación al entorno.

4. Unos operadores genéticos.

5. Una condición de terminación que determine cuando el proceso genético ha encontrado una solución óptima y no puede mejorar más la adaptación de los individuos al medio.

En la construcción del algoritmo genético (AG) para el problema de optimización que nos concierne se ha utilizado la librería GALib (Wall1999), la cual, por su carácter modular, permite el diseño por parte del usuario de todas o alguna de las etapas realizadas por el algoritmo. Esta librería ofrece la posibilidad de trabajar con diferentes tipos de algoritmos genéticos, en nuestro caso se ha optado por un AG de Estado Estacionario (AGEE), en el que se han adaptado las etapas de inicialización, evaluación y terminación.

\subsubsection{Codificación}

Tradicionalmente, las variables se han venido representado por cadenas binarias (Holland1975; Goldberg1989; Herrera1996). Sin embargo, en los últimos años diversos autores (Michalewicz1996; Fogel1995; Herrera1996) han investigado el uso de codificaciones distintas de la binaria, proponiendo el uso de representaciones más acordes con la naturaleza del problema, tales como la codificación real. En este ámbito, se han realizado múltiples estudios sobre la conveniencia de la utilización de un tipo de codificación u otra de los que se puede concluir que la elección de un método u otro depende del tipo de problema concreto, no existiendo estudios concluyentes que determinen el mejor funcionamiento de la codificación binaria respecto de la real o viceversa.

La optimización de parámetros termohidráulicos supone la utilización de variables reales, por tanto, el mecanismo de codificación seleccionado ha sido el real puesto que proporciona una representación más natural de los parámetros. El GALib permite utilizar ambas codificaciones, real y binaria. En la codificación utilizada por GALib, los parámetros del modelo a optimizar se organizan dentro de un vector de variables reales e independientes $\mathbf{x}=\left\{\mathrm{x}_{1}, \ldots, \mathrm{x}_{\mathrm{i}}, \ldots, \mathrm{x}_{\mathrm{n}}\right\} \in \mathbb{R}^{\mathrm{n}}$, donde a cada variable real, $\mathrm{x}_{\mathrm{i}}$, se le pueden establecer directamente las restricciones explícitas que debe cumplir en el momento de definir el vector. Estas restricciones incluyen aspectos relativos al tipo de variable, discreta o continua, y al rango de valores que puede tomar cada variable. En nuestro caso se va a trabajar con variables reales y continuas, acotando el rango de valores posibles de manera que el valor elegido tenga significado físico. 
Cada vector $\mathbf{x}$ va a contener una posible solución al problema de optimización. En la terminología de AAGG, cada solución se identifica con un individuo de la población, que se caracteriza por la información genética contenida en $\mathbf{x}$, por lo que a éste vector se le denomina genoma (cromosoma) y a cada variable $\mathrm{x}_{\mathrm{i}}$ se le denomina gen.

\subsubsection{Descripción del AGEE adaptado}

El esquema de funcionamiento del Algoritmo Genético de Estado Estacionario (AGEE) se muestra en la figura 3.1, donde se esquematizan las diferentes etapas que constituyen un ciclo evolutivo las cuales son descritas a continuación. El AGEE parte de una población base de forma que el número de individuos que constituyen esta población base, denominado popsize, lo fija el usuario. Cada uno de estos individuos constituye una posible solución del problema de optimización representada por la información genética contenida en su correspondiente genoma $\mathbf{x}$. Después de la reproducción de ciertos individuos seleccionados de la población base, el algoritmo genera una población auxiliar, de tamaño nrepl, que desciende de la inicial. La descendencia generada se evalúa y se añade a la población inicial obteniendo una población de tamaño popsize+nrepl. Cada individuo de la población resultante se escala con el fin de obtener una población ordenada en base a su aptitud.

Tras el escalado, los nrepl peores individuos son eliminados de la población con el fin de obtener una nueva población final de igual tamaño a la inicial, popsize, formada por los individuos mejor adaptados, la cual constituye la siguiente generación, representada genéricamente como g+1, que ha evolucionado desde la población anterior, g. El número de individuos a reemplazar nrepl lo fija el usuario, ajustando de este modo el grado de solapamiento deseado entre las poblaciones de dos generaciones consecutivas. Una vez generada la nueva población el algoritmo comprueba si se verifica el criterio de terminación y en caso negativo continúa con el proceso evolutivo para obtener la siguiente generación de la manera descrita anteriormente. De esta forma, el AGEE acumula información acerca de un espacio inicial de búsqueda desconocido y explota esta información para guiar las siguientes búsquedas en subespacios útiles. 


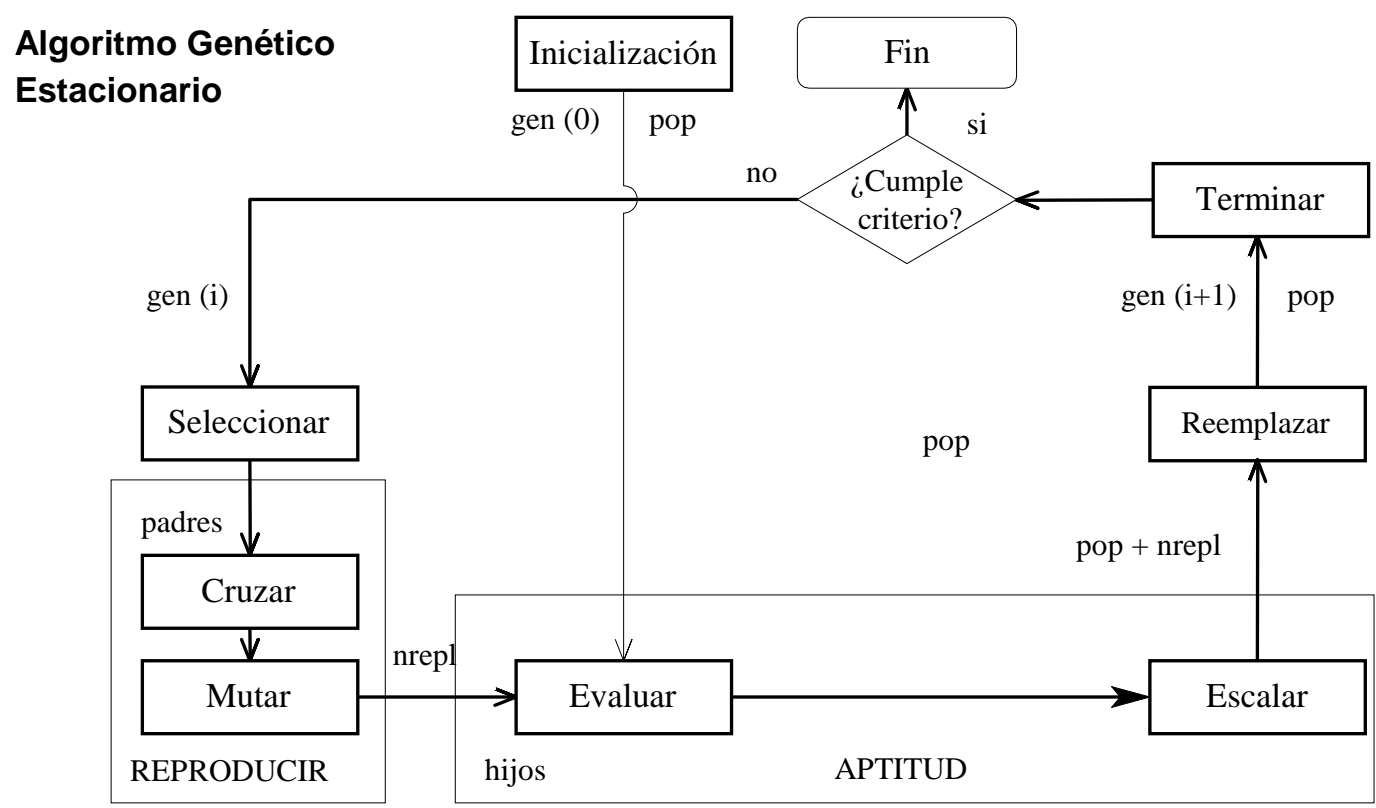

Figura 3.1: Ciclo evolutivo de un AGEE.

Una descripción más detallada de las etapas anteriores se realiza en los siguientes apartados.

\subsubsection{Inicialización}

El primer paso en la cadena de evolución consiste en la creación de una población inicial de un tamaño dado, popsize, la cual se trata de forma diferente a las demás $(\mathrm{g}=0)$, tal y como se observa en la figura 3.1 puesto que pasa directamente a ser evaluada.

La selección de un número idóneo de individuos de la población, popsize, constituye un aspecto importante en el comportamiento del algoritmo genético. Así, con poblaciones de tamaño pequeño se corre el riesgo de no cubrir de forma adecuada todo el espacio de búsqueda. Por el contrario, trabajar con poblaciones excesivamente grandes supone un coste computacional excesivo, que en muchas ocasiones resulta innecesario (Miller1996). Se han realizado diversos experimentos con la finalidad de obtener un tamaño adecuado para la población, llegando a la conclusión de que para la mayoría de los problemas considerados, un tamaño de la población entre 50 y 100 individuos proporciona buenos resultados (Goldberg1989). 


\subsubsection{Selección}

Después de la inicialización, el algoritmo entra en el bucle de evolución esquematizado en la figura 3.1. El bucle evolutivo en las siguientes generaciones ( $\mathrm{g}>0$ ) empieza con la selección de algunos de los individuos de la población base para ser los padres en el proceso de reproducción encaminado a obtener hijos. Por tanto, el objetivo del proceso de selección consiste, básicamente, en dirigir el proceso de búsqueda a favor de los individuos más aptos. De entre los diversos métodos disponibles en GALib para realizar este proceso de selección se ha escogido la selección por torneo. El criterio de selección por torneo es un método estocástico de muestreo, extensión del método de la ruleta (Goldberg1989). En los dos métodos se asigna a cada individuo de la población una probabilidad de ser seleccionado en función de su aptitud relativa respecto a los demás individuos de la población. La probabilidad asociada con un individuo i viene dada por:

$$
\mathrm{p}(\mathrm{i})=\frac{\varphi(\mathrm{i})}{\sum_{\mathrm{i}=1}^{\operatorname{popsize}} \varphi(\mathrm{i})} ; \quad \forall \mathrm{i}=1, \ldots, \text { popsize }
$$

donde $\mathrm{p}(\mathrm{i})$ representa la probabilidad que tiene el individuo i-ésimo de ser elegido y $\varphi(\mathrm{i})$ corresponde al valor de la aptitud, que corresponde con el valor de la función objetivo escalado, de dicho individuo. Lógicamente, los valores de las probabilidades calculadas son números comprendidos entre 0 y 1 .

El método de selección por torneo utiliza la ruleta para seleccionar un grupo de dos, o más, individuos desde la población base y selecciona aquel individuo del grupo que da un mejor resultado. Así, siguiendo la filosofía de la naturaleza, los mejores individuos se reproducirán más y crearan una mejor descendencia la cual contendrá parte de su información genética. Por tanto, las mejores soluciones predominarán en la descendencia y su información genética pasará a la siguiente generación.

\subsubsection{Reproducción}

La reproducción tiene por objeto la obtención de nuevos individuos mediante la utilización de los denominados operadores genéticos. Todo algoritmo genético hace uso de, al menos, dos operadores genéticos en la fase de reproducción: cruce y mutación (Goldberg1989).

El operador cruce combina los padres para obtener hijos con información genética de las mismas características que la de sus progenitores de esta manera se asegura que las características genéticas de los padres se transmiten a los hijos. De entre los operadores de cruce posibles, uno de los más utilizados es el cruce por un punto, el cual ha sido seleccionado en nuestro caso. Este tipo de cruce consiste en elegir al azar una posición en 
el genoma (vector $\mathbf{x}$ ) de los dos padres e intercambiar los segmentos en los que han quedado divididos para generar así dos nuevos hijos como se ilustra en la figura 3.2. Habitualmente, el cruce no se aplica a todas las parejas seleccionadas para cruzar, sino que se realiza una selección aleatoria en función de la probabilidad de cruce elegida por el usuario. En problemas donde la variabilidad de los genes es pequeña la probabilidad de cruce debe tomar valores elevados con el fin de evitar una excesiva clonación de los padres. Los valores recomendados (Bäck1996) para la probabilidad de cruce en este tipo de problemas oscilan entre 0.6 y 0.99 .

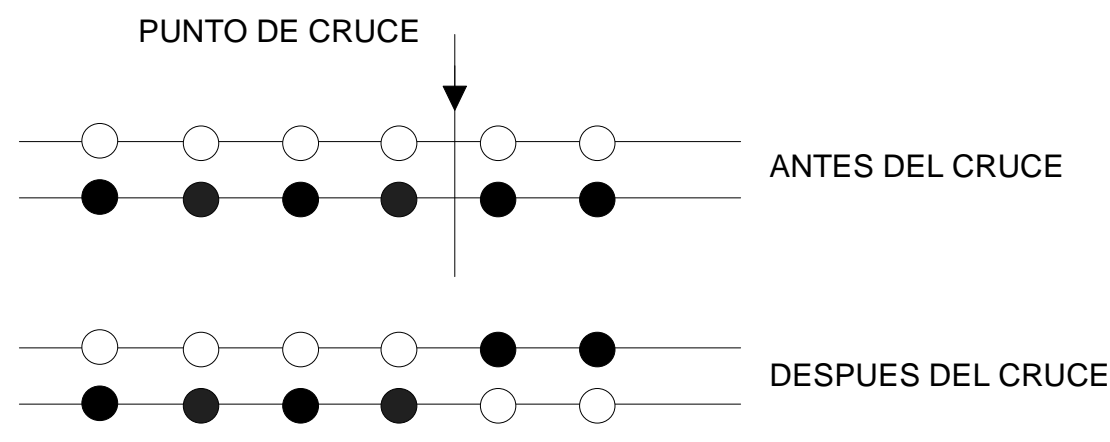

Figura 3.2: Cruce por un punto.

El operador mutación actúa sobre un individuo modificando su información genética. La alteración del genoma proporciona una garantía de accesibilidad a todos los elementos del espacio de búsqueda. Entre los diferentes tipos de operadores de mutación disponibles en el GALib se ha seleccionado el "flip mutator". Este mutador actúa eligiendo aleatoriamente una posición del genoma (vector $\mathbf{x}$ ) del individuo, padre o hijo, que puede sufrir la mutación. Para llevar a cabo la mutación se procede cambiando el valor del gen de esta posición (variable $\mathrm{x}_{\mathrm{i}}$ ) por otro valor que satisface las mismas restricciones explícitas que el anterior, el esquema de la mutación se muestra en la figura 3.3.

No obstante, la mutación no se da en todos los casos, sino que, al igual que en el caso del cruce el usuario establece una probabilidad de mutación para cada individuo de la población.

Respecto al valor adecuado para la probabilidad de mutación, Bäck (Bäck1996) sugiere que para cadenas binarias y funciones unimodales el valor óptimo de la probabilidad de mutación debe ser inversamente proporcional a la longitud de la cadena, $n$, es decir $1 / n$, estando comprendido dicho valor para el caso de funciones multimodales en el intervalo $[1 / \mathrm{n}, 1]$. Aunque es difícil extrapolar los resultados a nuestro problema, donde la codificación es real, tras diversos ensayos se ha comprobado que utilizando una 
probabilidad de mutación inversamente proporcional a la longitud del genoma $\mathbf{x}$ se obtienen buenos resultados (Herrera1996).

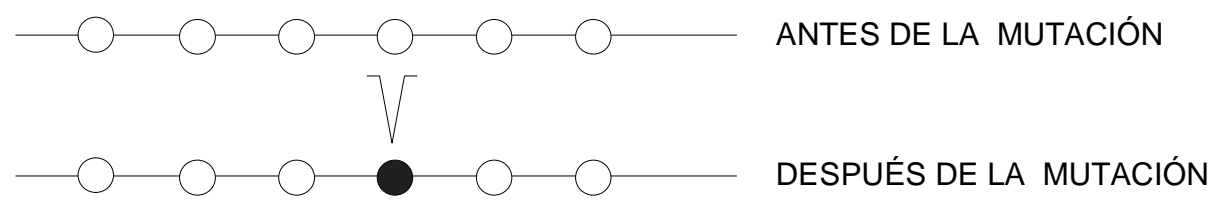

Figura 3.3: Proceso de mutación "Flip mutator".

\subsubsection{Evaluación}

Concluido el proceso de reproducción, el siguiente paso en el proceso de evolución corresponde a la evaluación de los nuevos individuos de la población según la función objetivo específica del problema, representada como f(i) para el individuo i-ésimo. En el caso que nos ocupa la función objetivo viene determinada por el error existente entre el cálculo proporcionado por el modelo termohidráulico y la referencia suministrada para cada variable.

\subsubsection{Escalado}

Una vez evaluada la función objetivo para cada uno de los individuos de la población, de tamaño popsize+nrepl, con respecto a la función objetivo, el bucle de la figura 3.1 continúa con la etapa de escalado, que pretende evitar dos problemas importantes que pueden darse durante la evolución del AG (Goldberg1989). El primero de ellos consiste en la aparición en las primeras generaciones de individuos muy buenos, llamados superindividuos, entre una población de individuos mediocres, lo que al dejar evolucionar libremente esta situación llevaría en pocas generaciones a poblaciones constituidas en su mayor parte por superindividuos, provocando una convergencia prematura del algoritmo. El segundo de los problemas que puede presentarse en un determinado momento de la evolución del algoritmo, consiste en que se encuentre una situación en la que aunque la población presente una diversidad significativa entre los individuos que la forman, el valor medio de las aptitudes de los individuos esté próximo al valor de los mejores individuos, y por tanto las diferencias entre las aptitudes necesarias para que exista una mejora desaparecen y el proceso se convierte en una elección al azar entre los individuos mediocres.

Los problemas anteriores se solucionan realizando un escalado de los valores de la función objetivo, lo que lleva a definir la denominada función de aptitud. Con el escalado se 
intentan también controlar los efectos de la presión selectiva, amortiguándolos en las primeras fases de la evolución y acentuándolos en las últimas. Entre los diferentes mecanismos para realizar el escalado se ha seleccionado el escalado según una relación exponencial, el cual evalúa la aptitud de cada individuo i, representada por $\varphi(i)$, a partir del valor alcanzado por éste respecto a la función f(i), según la expresión:

$$
\varphi(i)=f(i)^{k}
$$

Donde k es un número entero que puede definir el usuario, aunque por defecto el GALib utiliza el valor $\mathrm{k}=1.0005$.

\subsubsection{Reemplazamiento}

Después de calcular la aptitud de cada uno de los individuos de la población, el algoritmo entra en la fase de reemplazamiento. Existen diferentes métodos para reemplazar los individuos de una población según el tipo de AG utilizado. En el caso del AGEE, en esta etapa se ordenan todos los individuos de la población, de tamaño popsize $+n r e p l$, en base a su aptitud, para seguidamente eliminar de la población los nrepl individuos con peor aptitud, con lo que se consigue una población resultante del mismo tamaño que la inicial, es decir, de tamaño popsize, que incluye a los mejores individuos encontrados tras la evolución desde la generación anterior g hasta la nueva generación g+1.

\subsubsection{Terminación}

El proceso evolutivo anteriormente descrito se repite hasta que se cumple un determinado criterio de terminación, el cual especifica las condiciones que se han de cumplir para que se considere que el AGEE ha dado una solución aceptable, o en su defecto, ha agotado el número de intentos de búsqueda sin éxito.

En la literatura (Beasley1993a; Beasley1993b; Carlson1995) aparecen diferentes criterios de terminación. El más simple y utilizado consiste en limitar el número máximo de generaciones, $\mathrm{G}$, de manera que el algoritmo acaba tras evolucionar durante $\mathrm{G}$ generaciones, tanto si aún no ha encontrado una buena solución como si ya la había encontrado en alguna generación muy anterior. Otro método de terminación ampliamente utilizado se basa en la búsqueda de la convergencia de la mayor parte de los individuos, bien hacia un mismo valor respecto de la función objetivo o de la aptitud, bien hacia una misma información genética (mismo genoma o vector $\mathbf{x}$ ), o bien en ambas condiciones. El primer tipo se denomina convergencia poblacional y el segundo convergencia genética (o 
diversidad). En nuestro caso, el criterio de terminación diseñado comprueba los tres criterios:

1) número máximo de generaciones $(\mathrm{G})$,

2) convergencia poblacional (pconv), y

3) convergencia genética (popdiv), tal como se muestra en la figura 3.4.

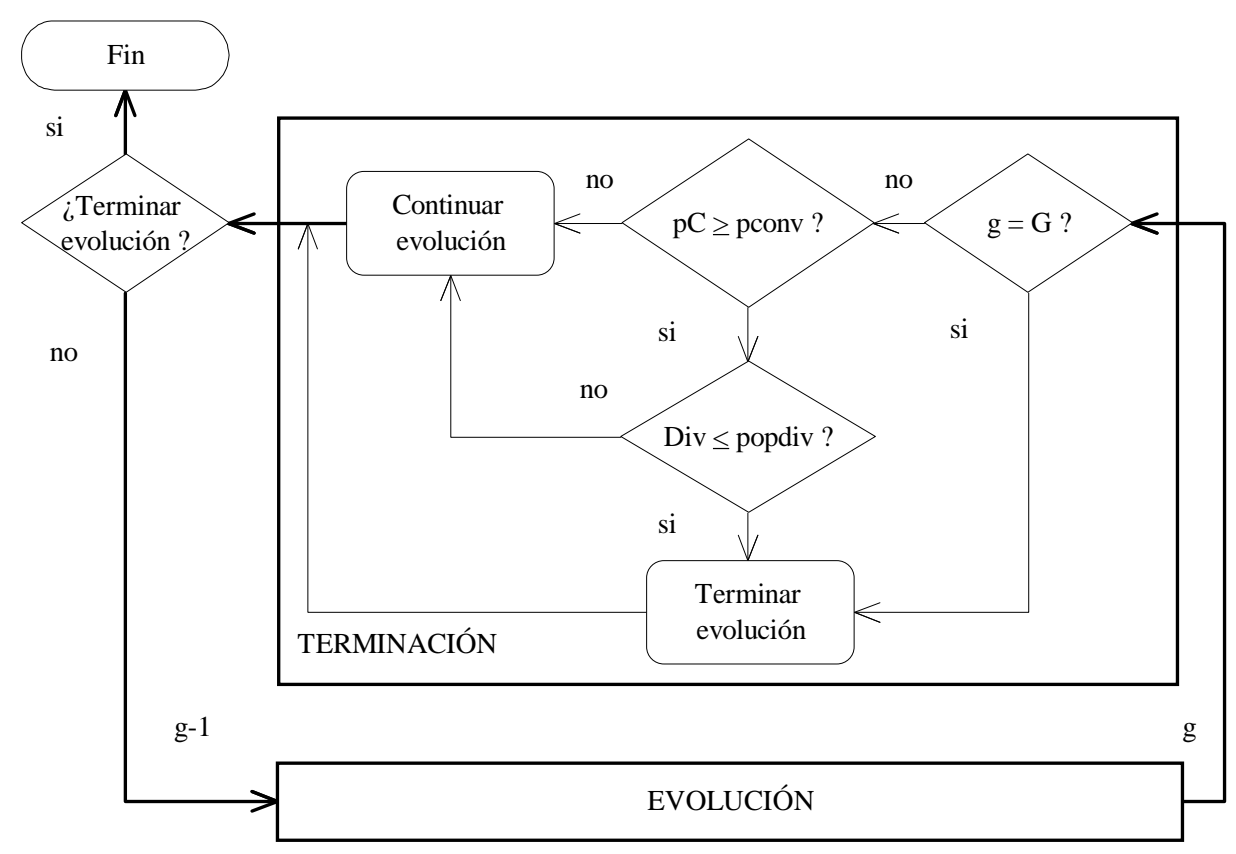

Figura 3.4: Criterio de terminación.

La primera comprobación que realiza el criterio de terminación consiste simplemente en comparar la generación actual, g, frente al número máximo de generaciones, $\mathrm{G}$, impuestas por el usuario. En el caso de que la generación sea igual al límite impuesto, el algoritmo parará. En caso contrario se procede a la evaluación de la convergencia de la población. En primer lugar se comprueba la convergencia poblacional, la cual se determina en función de los valores alcanzados por los individuos de la población respecto a su función de aptitud. El criterio de terminación por convergencia poblacional cuando se minimiza la función objetivo se evalúa como:

$$
\mathrm{pC}=1-\frac{|\varphi(\mathrm{m})-<\varphi(\mathrm{i})>|}{<\varphi(\mathrm{i})>} \geq \mathrm{pconv}
$$


donde $\varphi(\mathrm{m})$ es el valor mínimo de aptitud, que ha sido obtenido por el individuo m-ésimo, $\langle\varphi(i)>$ corresponde a la media de las aptitudes obtenidas por los individuos de la población en la generación actual y pconv es el nivel de convergencia fijado por el usuario.

Con arreglo al esquema de la figura 3.4, en caso de no cumplirse la condición $\mathrm{pC} \geq \mathrm{pconv}$, el AGEE continúa con el proceso evolutivo. De otro modo, se procede a comprobar la diversidad de la población; es decir, se comprueba la convergencia de los genomas de los individuos, analizando si contienen la misma información genética (mismo vector $\mathbf{x}$ ). La diversidad de la población se evalúa comparando entre sí todos los individuos que la constituyen utilizando una adaptación de la distancia de 'Hamming'(Bäck1996). El proceso se repite para todos los individuos de la generación en la que se encuentra el algoritmo con el fin de evaluar la diversidad de la población como sigue:

$$
\operatorname{Div}=\frac{2}{\text { popsize (popsize }-1)} \cdot \sum_{\mathrm{i}=1}^{\text {popsize }} \sum_{\mathrm{j}=1}^{\mathrm{i}-1} \frac{\sum_{\mathrm{k}=1}^{\mathrm{L}} \boldsymbol{\delta}_{\mathrm{i}_{\mathrm{k}}, \mathrm{j}_{\mathrm{k}}}}{\mathrm{L}} \leq \text { popdiv }
$$

donde popdiv es la máxima diversidad permitida por el usuario, $\mathrm{i}_{\mathrm{k}} \mathrm{e} \mathrm{j}_{\mathrm{k}}$ corresponden a las componentes k-ésimas (gen k) de los vectores (genomas) i y j respectivamente, los cuales caracterizan a los dos individuos comparados, cuya longitud L coincide con el número de variables (genes) codificados en un genoma, evaluándose además:

$$
\delta_{i_{k}, j_{k}}= \begin{cases}0, & \text { si }\left|i_{k}-j_{k}\right|<\varepsilon \\ 1, & \text { de otro modo }\end{cases}
$$

donde $\varepsilon$ establece el nivel de precisión, o tolerancia máxima admisible, en la comparación del valor de dos genes idénticos de distintos individuos, por lo que toma valores muy pequeños y próximos a cero.

En caso de no verificarse el criterio de diversidad establecido mediante la ecuación 3.5, el algoritmo AGEE continúa con el proceso evolutivo, y termina en caso contrario, tal como se muestra en la figura 3.4.

Resulta interesante comprobar la diversidad de la población porque podría llegarse a una situación en la que la población estuviese formada por muchos individuos diferentes con valores para su función de aptitud muy parecidos, y por lo tanto se verificaría la convergencia poblacional pero no convergería la información genética de los individuos, por lo que no se puede asegurar que se ha alcanzado el óptimo, puesto que al dejar evolucionar esta situación es posible que alguno de los individuos pueda evolucionar a un valor mejor. Este aspecto resulta especialmente interesante para funciones con un único 
óptimo global puesto que al terminar el algoritmo la población debería estar formada por individuos con la misma información genética ajustada a este óptimo.

El criterio de terminación presentado tiene la ventaja que se puede definir un número máximo de generaciones muy elevado sin que esto suponga un excesivo coste en tiempo de computación puesto que si el algoritmo alcanza la convergencia en una generación anterior termina la evolución. No obstante, hay que señalar que en ocasiones el cálculo de la diversidad requiere un tiempo de cálculo importante, sobre todo cuando son muchos los parámetros codificados en $\mathbf{x}$ que hay que optimizar; es decir, cuando la longitud L del genoma es muy elevada. No obstante, fijar el número de generaciones máximo, G, garantiza la finalización del AGEE aún en casos donde hay varios óptimos locales, lo que implica la imposibilidad de alcanzar la convergencia genética como consecuencia de que distintos individuos con información genética distinta tienen un mismo valor de la función de aptitud.

\subsection{Algoritmos de búsqueda directa}

Los algoritmos de búsqueda directa se caracterizan por el hecho de que el proceso de toma de decisiones se basa solamente en la información que proporciona el valor de la función; en estos algoritmos no se requiere la información suministrada por la derivada para el cálculo de la dirección de decrecimiento de la función. De entre los métodos de búsqueda directa cabe señalar: el algoritmo símplice de Nedler-Mead (Kelley1999), el algoritmo de Hooke-Jeeves (Kelley1999) y el algoritmo de búsqueda multidireccional (MDS) (Kelley1999).

Los métodos directos de búsqueda son muy utilizados en la práctica puesto que se puede estar trabajando con datos en los que no sea posible el cálculo de la derivada, como suele suceder si se trabaja con datos experimentales. Además las funciones basadas en este tipo de datos contienen normalmente ruido por lo que las aproximaciones mediante diferencias finitas a la derivada de la función objetivo pueden parecer poco realistas. Finalmente, la mayoría de los métodos de búsqueda son fáciles de comprender, de implementar y de usar.

Por otro lado, los algoritmos de búsqueda directa presentan desventajas como, en ocasiones, la carencia de una teoría de convergencia. La convergencia excesivamente lenta en puntos cercanos a la solución o incluso la no convergencia cuando el problema tiene una dimensión considerable, más de 10 incógnitas. No obstante pese a estos problemas siguen siendo métodos de búsqueda muy utilizados. 
De entre los métodos de búsqueda existentes, hemos optado por el método de búsqueda multidireccional por su sencillez y buen funcionamiento, además de contar con una teoría de convergencia.

\subsubsection{Descripción del algoritmo de búsqueda multidireccional (MDS)}

El algoritmo de búsqueda multidireccional puede considerarse como un método que utiliza una línea como estrategia global de optimización. Dada esta caracterización del algoritmo necesitamos definir:

- Un método para obtener la dirección de búsqueda que garantice un decrecimiento en el valor de la función en una iteración determinada, para un paso de tiempo y una longitud de los lados.

- Un método para obtener la longitud adecuada del paso.

Para determinar la longitud de búsqueda algunos métodos directos exploran en cada iteración cada una de las $\mathrm{n}$ direcciones linealmente independientes, siendo $\mathrm{n}$ la dimensión del problema de optimización. Si la función objetivo es diferenciable al menos existe una dirección ortogonal al gradiente que determina la dirección de descenso, si además el paso tomado en esta dirección es suficientemente pequeño el descenso está garantizado.

En una iteración $\mathrm{k}>0$ el algoritmo de búsqueda multidireccional (MDS) requiere $n+1$ puntos, $\mathrm{V}_{0}{ }^{\mathrm{k}}, \ldots, \mathrm{V}_{\mathrm{n}}{ }^{\mathrm{k}}$ que definan un símplice no degenerado en $\mathbb{R}^{\mathrm{n}}$, esto es, las aristas del símplice son $n$ vectores linealmente independientes. Se entiende por no degenerado que el conjunto de $\mathrm{n}$ lados adyacentes a un vértice del símplice se expande en $\mathbb{R}^{\mathrm{n}}$. En la figura 3.5 se considera el caso bidimensional en el que el símplice es un triángulo formado por los vértices $\left\langle\mathrm{V}_{0}{ }^{\mathrm{k}}, \mathrm{V}_{1}{ }^{\mathrm{k}}, \mathrm{V}_{2}{ }^{\mathrm{k}}\right\rangle$. Los lados del símplice se utilizan para definir las direcciones de búsqueda, la orientación de las direcciones de búsqueda y el tamaño del paso en cada dirección. 


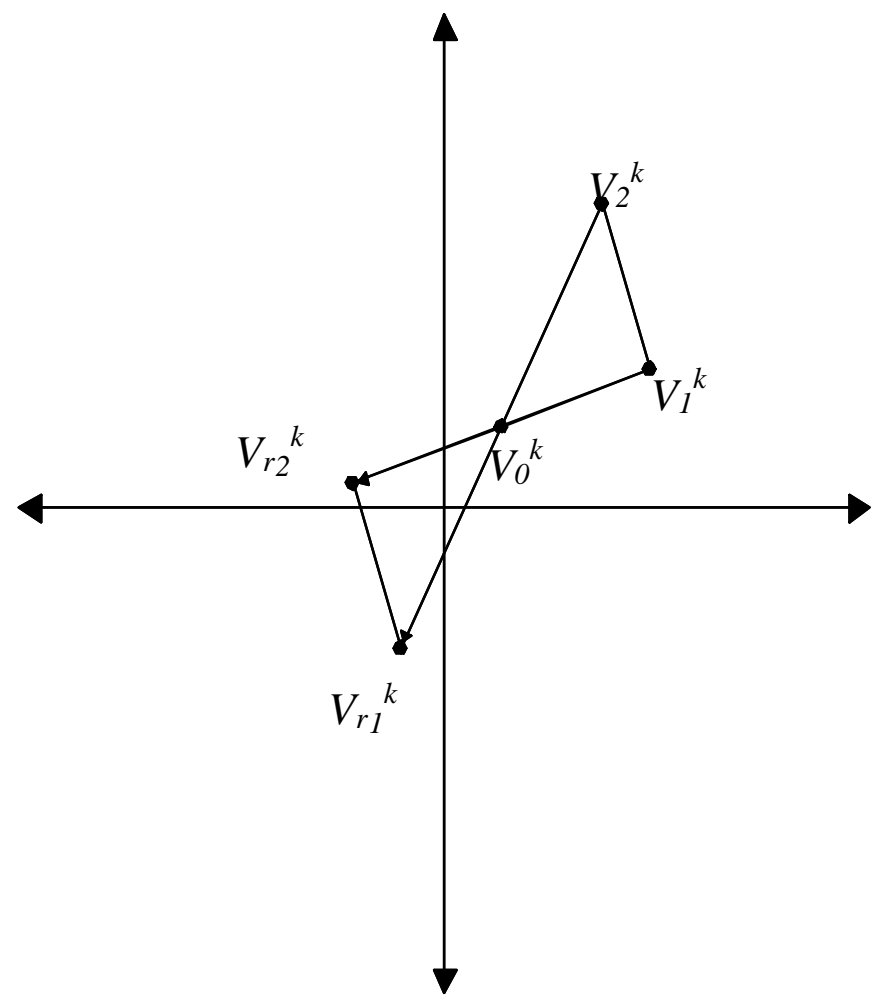

Figura 3.5: Símplice original y su reflexión.

El método empieza calculando el valor de la función para cada uno de los $n+1$ vértices del símplice original. Utilizando la información proporcionada por el valor de la función, el algoritmo escoge el mejor vértice del símplice, entendiendo por mejor el que tenga el valor más pequeño de la función objetivo. Utilizando la notación que toma $\mathrm{V}_{0}{ }^{\mathrm{k}}$ como el mejor vértice en una determinada iteración $\mathrm{k}$, entonces tenemos que el mejor vértice satisface:

$$
f\left(V_{0}{ }^{k}\right) \leq f\left(V_{i}^{k}\right) \text { para } i=1, \ldots, n .
$$

Como ya se ha comentado, los $\mathrm{n}$ lados que conectan el mejor vértice con los otros $\mathrm{n}$ vértices determinan un conjunto de $\mathrm{n}$ direcciones linealmente independientes. La figura 3.5 muestra estas direcciones que vienen dadas por $\mathrm{V}_{0}{ }^{\mathrm{k}} \mathrm{V}_{1}{ }^{\mathrm{k}} \mathrm{y} \mathrm{V}_{0}{ }^{\mathrm{k}} \mathrm{V}_{2}{ }^{\mathrm{k}}$.

A partir de este momento empieza el proceso de optimización en el que se siguen tres etapas básicas: la reflexión, la expansión y la contracción, que se describen a continuación. El proceso completo que sigue el método MDS viene esquematizado en la figura 3.6. 


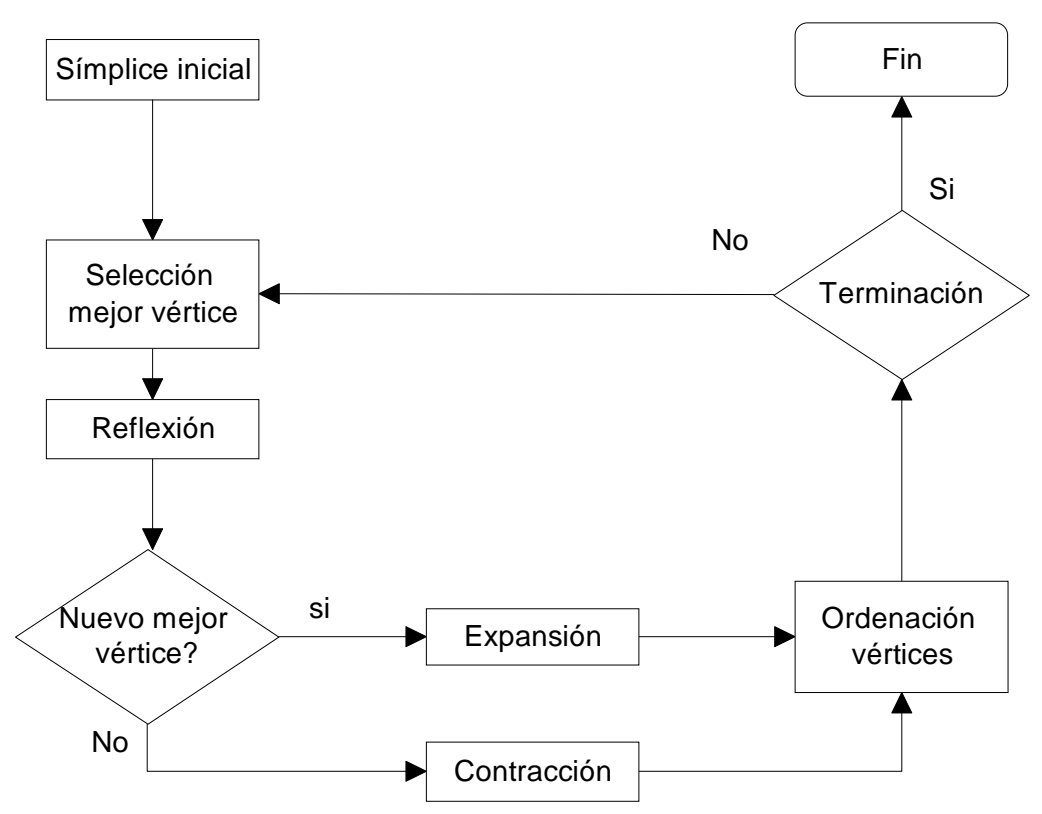

Figura 3.6: Esquema del método MDS.

\subsubsection{Etapa de reflexión}

En esta fase, y partiendo del mejor vértice encontrado, $\mathrm{V}_{0}{ }^{\mathrm{k}}$, el algoritmo hace un paso en cada una de las $n$ direcciones. La longitud de cada paso es igual a la del lado que determina la dirección de búsqueda. Geométricamente este proceso se explica como una reflexión del símplice original por el mejor vértice para dar un nuevo símplice. El resultado de esta fase se observa en la figura 3.5 donde el nuevo símplice viene dado por los vértices, $\left\langle\mathrm{V}_{0}{ }^{\mathrm{k}}, \mathrm{V}_{\mathrm{r} 1}{ }^{\mathrm{k}}, \mathrm{V}_{\mathrm{r} 2}{ }^{\mathrm{k}}\right\rangle$ que comparte únicamente con el original, $\left\langle\mathrm{V}_{0}{ }^{\mathrm{k}}, \mathrm{V}_{1}{ }^{\mathrm{k}}, \mathrm{V}_{2}{ }^{\mathrm{k}}\right\rangle$, el mejor vértice, $\mathrm{V}_{0}{ }^{\mathrm{k}}$. Los ángulos y las longitudes de los lados del nuevo símplice de prueba son los mismos que los del original.

En este paso se considera que se ha tenido éxito si se satisface que alguno de los nuevos vértices tiene un valor de la función mejor que el valor de la función en el mejor vértice del símplice original. Por tanto, debe satisfacerse la condición:

$$
\min \left\{f\left(\mathrm{~V}_{\text {ri }}^{\mathrm{k}}\right), \mathrm{i}=1, \ldots, \mathrm{n}\right\}<\mathrm{f}\left(\mathrm{V}_{0}^{\mathrm{k}}\right)
$$

La razón para exigir esta condición se encuentra en el hecho de que en caso contrario el mejor vértice para en nuevo símplice seguiría siendo el original, con lo que al generar una nueva reflexión estaríamos de nuevo en la condición inicial, como puede observarse en la figura 3.5. 
La comprobación del criterio de aceptación de este paso supone la evaluación de los n nuevos vértices, $\mathrm{V}_{\mathrm{r} 1}{ }^{\mathrm{k}}, \ldots, \mathrm{V}_{\mathrm{rn}}{ }^{\mathrm{k}}$. Llegados a este punto y cualquiera que haya sido la respuesta a la condición de éxito de la reflexión se considera una nueva prueba que consiste en expandir o contraer el símplice.

\subsubsection{Etapa de expansión}

Supongamos que ninguno de los nuevos vértices definidos en la etapa anterior satisface los criterios de aceptación establecidos en el problema, entonces se plantea el problema de si se encontrará o no mejoras incluso considerando distancias más grandes entre los puntos. Para hacer esta comprobación el algoritmo empieza otra vez desde el mejor vértice, $\mathrm{V}_{0}{ }^{\mathrm{k}}$, en cada una de las $\mathrm{n}$ direcciones originales de búsqueda, pero ahora utilizando un paso de longitud el doble del original.

Geométricamente, como puede verse en la figura 3.7, el algoritmo "expande" el símplice de prueba doblando la longitud de cada uno de los lados en el símplice reflejado para obtener el nuevo símplice de prueba $\left\langle\mathrm{V}_{0}{ }^{\mathrm{k}}, \mathrm{V}_{\mathrm{e} 1}{ }^{\mathrm{k}}, \mathrm{V}_{\mathrm{e} 2}{ }^{\mathrm{k}}\right\rangle$. Cabe señalar que el símplice definido en este paso tiene los ángulos iguales a los del símplice original, sin embargo las longitudes de los lados están reescaladas al doble.

El criterio de aceptación de esta etapa se basa en el razonamiento de que no es necesario incrementar el paso del símplice a no ser que en esta etapa se mejore el decrecimiento en los valores de la función con respecto a los del símplice inicial. Esto nos lleva a plantear el siguiente criterio de aceptación:

$$
\min \left\{f\left(V_{e i}{ }^{k}\right), i=1, \ldots, n\right\}<\min \left\{f\left(V_{r i}{ }^{k}\right), i=1, \ldots, n\right\}
$$

Si se satisface el criterio de aceptación, la nueva iteración empieza con el símplice definido por $\left\langle\mathrm{V}_{0}{ }^{\mathrm{k}}, \mathrm{V}_{\mathrm{el}}{ }^{\mathrm{k}}, \ldots, \mathrm{V}_{\mathrm{en}}{ }^{\mathrm{k}}\right\rangle$, siempre que se cumplan también los criterios establecidos en la definición del problema. En caso contrario la nueva etapa del método se realiza a partir del símplice reflejado $\left\langle\mathrm{V}_{0}{ }^{\mathrm{k}}, \mathrm{V}_{\mathrm{r} 1}{ }^{\mathrm{k}}, \ldots, \mathrm{V}_{\mathrm{rn}}{ }^{\mathrm{k}}\right\rangle$. 


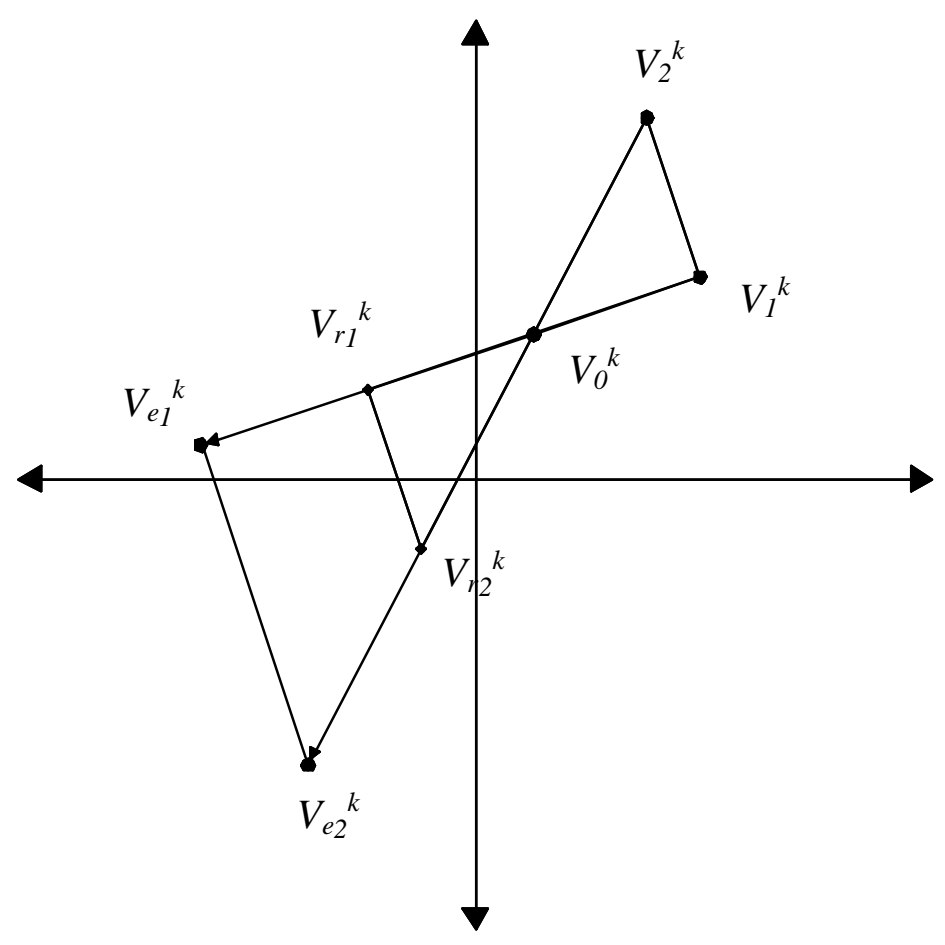

Figura 3.7: Símplice original con el reflejado y su expansión.

\subsubsection{Etapa de contracción}

Si ninguno de los vértices del símplice reflejado satisface los criterios de aceptación planteado en el problema, se plantea la posibilidad de que los pasos utilizados sean demasiado grandes. Con el fin de verificar esta posibilidad se realiza otra vez la búsqueda con las mismas direcciones pero con pasos más pequeños. Esta acción se representa gráficamente en la figura 3.8, en la que puede observarse cómo simplemente el algoritmo "contrae" el símplice original hacia el mejor de los vértices encontrados, $\mathrm{V}_{0}{ }^{\mathrm{k}}$, dividiendo por dos cada uno de los lados del símplice original. El algoritmo empezará una nueva iteración con el símplice contraído $\left\langle\mathrm{V}_{0}{ }^{\mathrm{k}}, \mathrm{V}_{\mathrm{c} 1}{ }^{\mathrm{k}}, \ldots, \mathrm{V}_{\mathrm{cn}}{ }^{\mathrm{k}}\right\rangle$.

Antes de proceder con la nueva iteración se comprueba si el nuevo símplice satisface la condición de decrecimiento, es decir si se cumple que:

$$
\min \left\{f\left(V_{c i}{ }^{k}\right), i=1, \ldots, n\right\}<f\left(V_{0}{ }^{k}\right)
$$


Si la condición anterior se cumple indica que alguno de los nuevos vértices es mejor que el considerado hasta el momento, y la nueva iteración empieza a partir de este nuevo punto. En caso contrario, la nueva iteración se realiza a partir del mismo punto que la anterior, con las mismas direcciones de búsqueda, pero con las distancias entre los puntos la mitad que las originales.

De cualquier modo, tanto si se obtiene un nuevo vértice de partida como si no, en caso de que el algoritmo construya el símplice contraído, éste es el que se utiliza para empezar la siguiente iteración.

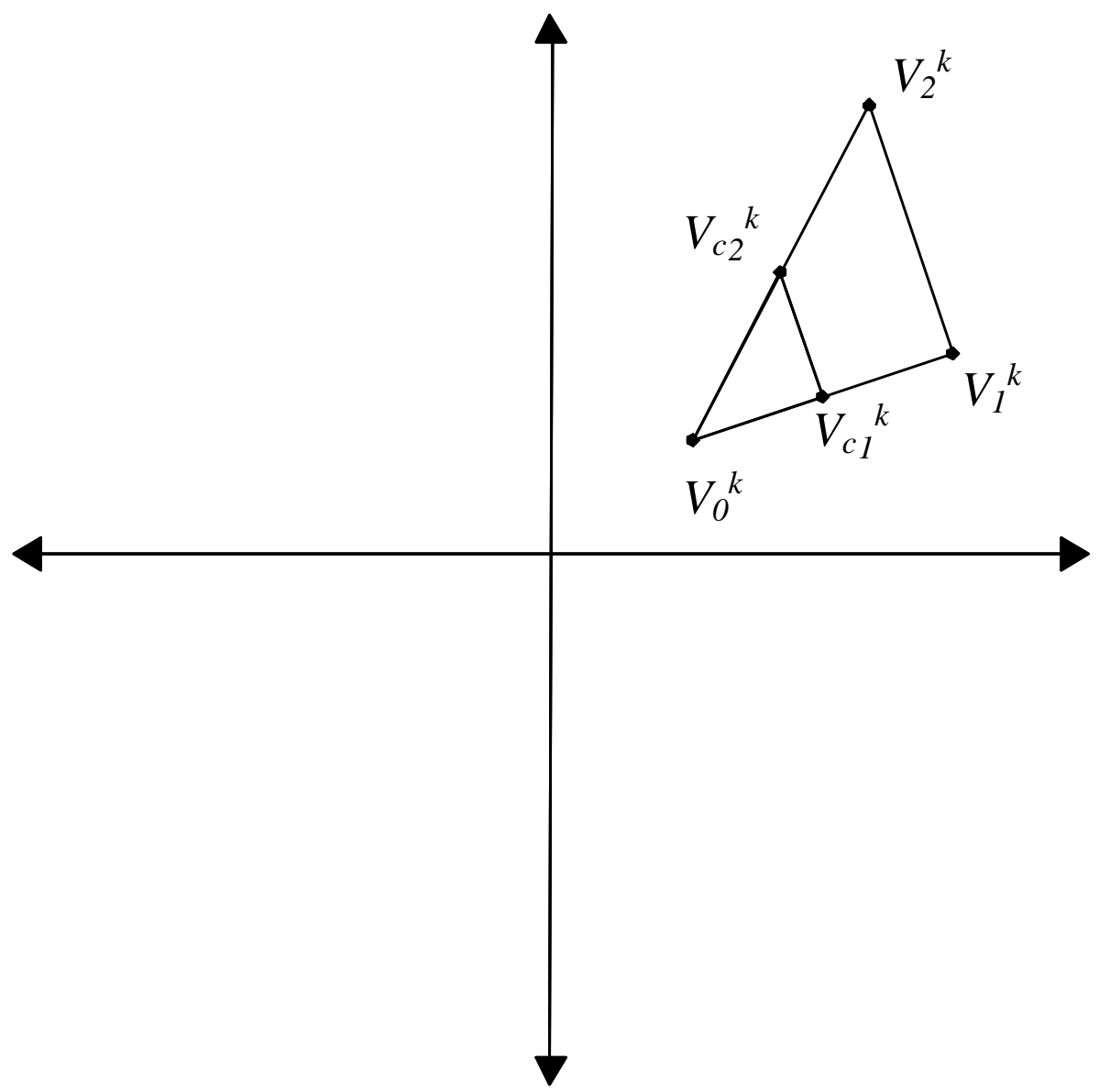

Figura 3.8: Símplice original y su contracción. 


\subsubsection{Nuevas direcciones de búsqueda y longitudes de pasos}

El objetivo del método de búsqueda multidireccional consiste en construir una sucesión de mejores vértices, $\left\{\mathrm{V}_{0}{ }^{\mathrm{k}}\right\}$, que converja a un punto crítico, mínimo preferiblemente, de la función $\mathrm{f}(\mathrm{V})$. Por esta razón se necesita que la sucesión de los valores de la función hacia el mejor valor sea monótona decreciente. Así, sólo se acepta un nuevo mejor vértice si satisface las condiciones de decrecimiento establecidas en cada una de las etapas que constituyen el método de búsqueda multidireccional.

Existen razones de tipo práctico para desear reemplazar el mejor vértice, puesto que la elección del mejor vértice determina las direcciones de búsqueda del algoritmo, puesto que éstas se definen como los n lados adyacentes al mejor vértice. Por esta razón cuando se plantea la posibilidad de elegir como nuevo símplice el reflejado, es imprescindible que se verifique la condición de haber encontrado un nuevo mejor vértice, puesto que de otro modo se daría el caso de estar en un bucle que nos devuelve siempre al símplice original y desde éste otra vez a su reflejado. En cambio, si se acepta el símplice reflejado o expandido, se garantiza que la próxima iteración empieza con un nuevo vértice y con n-1 nuevas direcciones de búsqueda, como puede observarse en la figura 3.9. Una de las direcciones permanece constante puesto que es la que une el nuevo mejor vértice con el mejor vértice anterior, por tanto no se descarta una dirección en la que se ha encontrado un descenso hasta que se observe un descenso mayor en otra dirección. Esta situación es especialmente satisfactoria si el símplice que se acepta es el expandido porque esto indica que se ha obtenido un descenso significativo del valor de la función. Por tanto, incluso si en la iteración se abandona la búsqueda en esta dirección, esta dirección se mantiene en la siguiente.

Si no se reemplaza el mejor vértice, y por tanto no cambian las direcciones de búsqueda, se divide la longitud del paso, lo que implica que también se divide la longitud del paso en el símplice reflejado de la siguiente iteración. El resultado de esta acción se muestra en la figura 3.10 donde se muestra el proceso de división de los pasos de búsqueda hasta que se cumplen las condiciones de aceptación de las etapas de reflexión o contracción del método. 


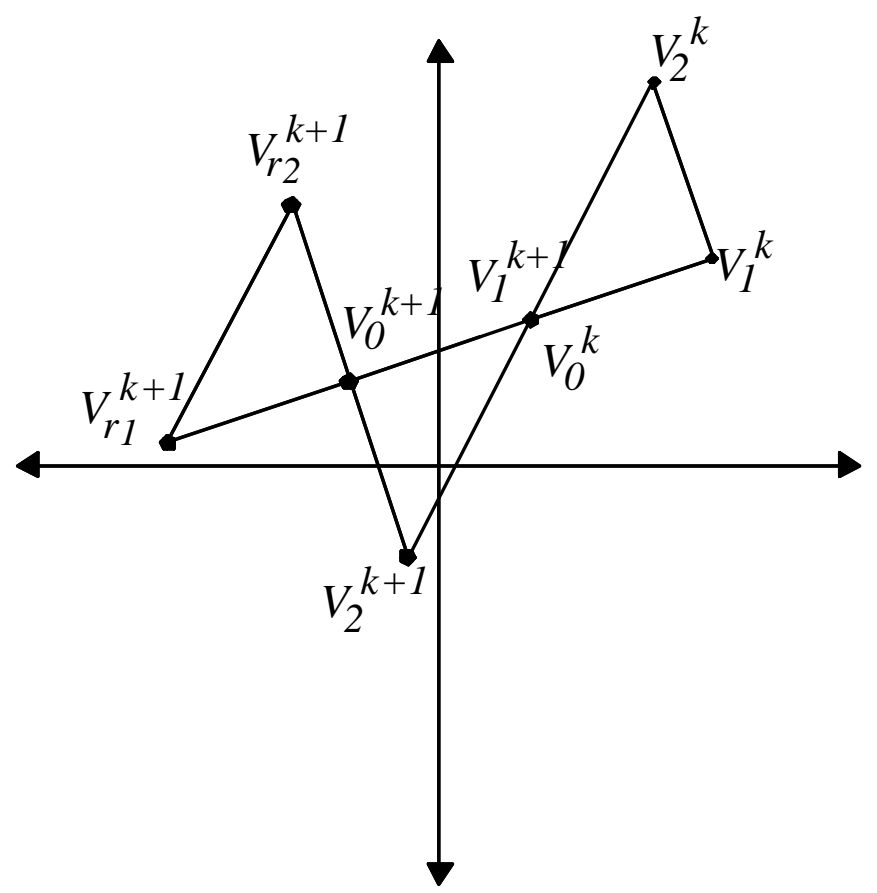

Figura 3.9: Nuevas direcciones de búsqueda en iteraciones consecutivas.

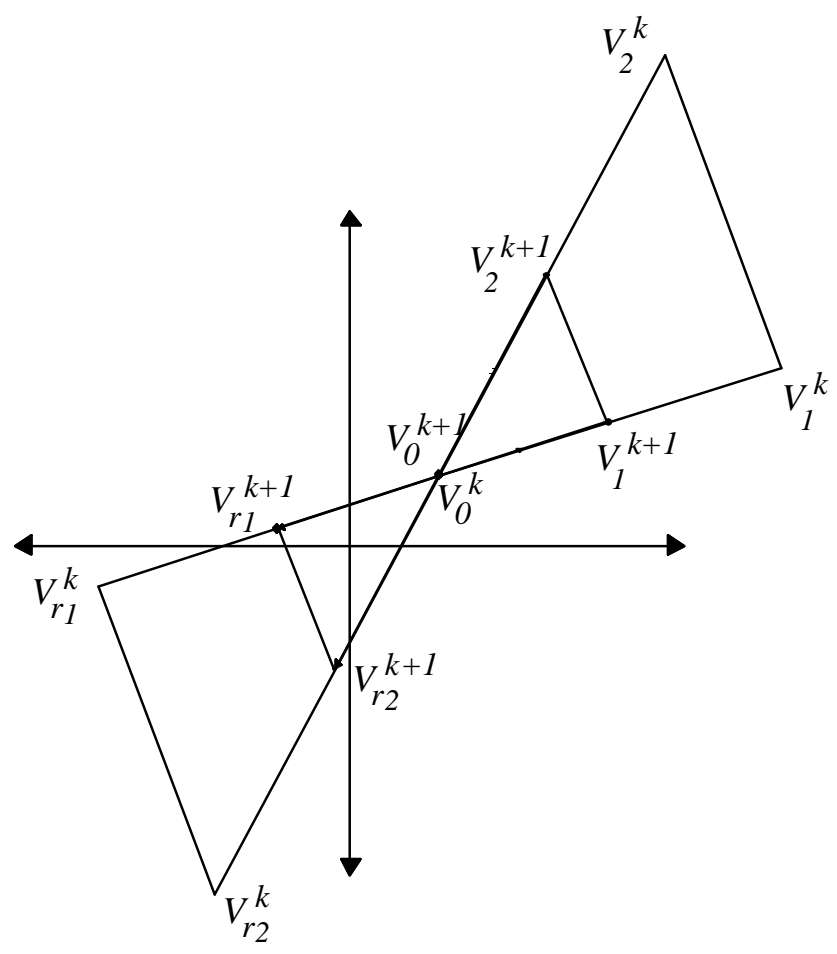

Figura 3.10: Efecto de nuevos pasos de búsqueda en iteraciones consecutivas. 
Con la expansión y contracción el algoritmo permite modificar los pasos de búsqueda, sin embargo, el paso real depende del símplice inicial que se ha seleccionado, puesto que los lados del símplice original determinan longitudes relativas de tamaño unidad, que después en las etapas de expansión y contracción son multiplicadas por los factores de escala definidos para cada una de estas etapas y que permanecen fijos durante todo el proceso de optimización. Además, los lados del símplice inicial determinan un conjunto de direcciones de búsqueda que se mantienen durante todas las iteraciones. Por tanto, el avance del método depende en gran medida de la selección del símplice inicial.

\subsubsection{Teorema de convergencia}

En general, una de las debilidades de los métodos de búsqueda directa es la teoría que garantiza la convergencia del método. Sin embargo, el método de búsqueda multidireccional cuenta con un análisis teórico de convergencia tanto para el problema libre como con restricciones lineales y de acotación de las variables (Torczon1989; Torczon1990; Torczon1999; Torczon2000), el cual sigue las líneas clásicas desarrolladas en los métodos que utilizan gradientes; sin embargo este análisis presenta la novedad de que el cálculo explícito del gradiente no es necesario en este método.

El proceso completo de prueba de la convergencia del método de búsqueda multidireccional consiste en demostrar tres puntos importantes:

- Comprobar que el método de búsqueda multidireccional es un método de descenso.

- Demostrar que las direcciones de búsqueda del método no se deterioran durante el proceso de optimización.

- Demostrar que el algoritmo garantiza un descenso suficiente en el valor de la función objetivo para el tamaño de paso que se ha tomado.

Con relación al primero de los puntos a demostrar, se puede observar que el algoritmo de no asegura un nuevo vértice en cada iteración, debido a que el mejor vértice definido no cambia hasta que se logra encontrar un vértice que produzca un descenso en el valor de la función objetivo, por tanto se puede garantizar que en cada iteración, o bien se mantiene el mejor vértice, o éste es reemplazado por otro mejor. Sin embargo, debido a la manera en la que actúan cada una de las etapas del algoritmo se demuestra que en un número finito que iteraciones el método es capaz de encontrar un nuevo mejor vértice, y por tanto satisface la definición de método en descenso (Kelley1999). 
En cuanto a las direcciones de búsqueda, debido a que el algoritmo de búsqueda multidireccional no cambia la forma original del símplice inicial, porque no varían los ángulos que lo forman, se puede garantizar que durante todo el proceso de optimización las direcciones de búsqueda son linealmente independientes y por tanto no se deterioran.

Por último, la estructura del algoritmo proporciona un mecanismo de control de las longitudes de los pasos puesto que en cada una de las etapas del MDS el paso que se utiliza está escalado con respecto a la longitud de los lados del símplice inicial. Más detalles técnicos sobre las demostraciones de estos puntos se encuentran en las referencias (Torczon1989; Torczon1990).

\subsubsection{Implementación}

En la implementación del algoritmo MDS aparecen algunos detalles que deben tenerse en cuenta para conseguir unos resultados satisfactorios en la optimización. Entre las características particulares de cada problema de optimización se debe tener en cuenta la selección de un símplice inicial adecuado, la selección de unos valores adecuados para los coeficientes de contracción y expansión, y la definición del criterio de terminación.

El algoritmo MDS requiere de un símplice inicial no degenerado a partir de cual empezar la búsqueda, que, para abordar un problema de dimensión $n$, se define a partir de $n+1$ puntos. La selección de la forma, el tamaño y la orientación del símplice depende del problema particular, aunque se pueden dar algunos criterios a seguir.

Del teorema de convergencia del algoritmo de búsqueda multidireccional se deduce que una solución es generar inicialmente un símplice regular (Torczon1989), entendiendo por regular que tenga las mismas longitudes de los lados, o se podría elegir un símplice con uno de sus ángulos recto. El símplice regular puede generarse definiendo cada uno de los $\mathrm{n}$ nuevos vértices a una determinada distancia fija en cada una de las n coordenadas en las que se va a iniciar la búsqueda. Esto es, dados los vectores de la base canónica $\mathrm{e}_{\mathrm{i}} \mathrm{y}$ los escalares $\alpha_{\mathrm{i}}$, y tomando como punto inicial $\mathrm{x}_{0}$ se construyen los restantes puntos como $\mathrm{x}_{\mathrm{i}}=\mathrm{x}_{0}+\alpha_{\mathrm{i}} \mathrm{e}_{\mathrm{i}}$. De este modo se garantiza que este símplice cubrirá todo el espacio mientras los $\alpha_{i}$ sean distintos de cero. En cualquier caso, y debido a que el símplice inicial se modifica rápidamente, se demuestra que su forma es un factor poco importante en el desarrollo del algoritmo.

Finalmente, en cuanto a la forma del símplice inicial debe tenerse en cuenta que depende de la escala del problema, por tanto si las variables difieran en cuanto a la escala puede no ser deseable la elección de un símplice regular. En esta situación puede ser preferible construir un símplice con un ángulo recto desde el punto inicial, y para cada una de las direcciones de búsqueda $e_{i}$, se toma un paso de tamaño $\alpha_{i}$, donde $\alpha_{i}$ es un factor de escala 
de la variable $\mathrm{x}_{\mathrm{i}}$. La ventaja que presenta el algoritmo de búsqueda multidireccional respecto a otros algoritmos de búsqueda directa es que el factor de escala utilizado para cada variable se mantiene durante todo el proceso de optimización.

En cuanto al tamaño del símplice inicial, es una característica que influye en los resultados del proceso de optimización, sin embargo, debido a la dependencia de la escala de las variables no es posible dar unas líneas generales a seguir para la elección de un símplice. En principio si el problema no está muy mal escalado se puede elegir un símplice regular con longitudes unidad de los lazos. En cualquier caso, el método MDS presenta la ventaja de que en las etapas de expansión y contracción se reajusta automáticamente el tamaño del símplice mediante el escalado de las longitudes de cada uno de los lados del mismo. Un problema que aparece si el tamaño del símplice es muy grande es que el algoritmo invierte una cantidad significante de iteraciones simplemente contrayendo el símplice sin realizar ningún progreso en la optimización, y en cada iteración que invierte para contraer se realizan 2 n evaluaciones de la función.

La orientación del símplice inicial determina las direcciones de búsqueda y por tanto es un factor que afecta al progreso del método. Aunque el problema de escoger la orientación depende en gran medida de la información que proporcione la función, aunque se recomienda que la opción más segura es la de empezar la optimización con un símplice regular (Torczon1989) puesto que de otro modo podría haber una convergencia más lenta hacia la solución del problema, aunque una mala selección de la orientación o de la escala del símplice inicial no provocan problemas graves en la manera de proceder del método MDS.

Hasta el momento al describir las etapas de expansión y contracción del método de búsqueda multidireccional se ha supuesto que las longitudes de los lados que determinan el símplice se multiplicaban o dividían por dos según se estuviera en las etapas de expansión o contracción. Sin embargo, en ningún caso se obliga a que tomen estos valores, de hecho las características que deben verificar estos parámetros son: ser números racionales, mayores que uno en el caso del coeficiente de expansión, y del intervalo ]0,1[ para el coeficiente de contracción.

Para cada problema en particular el valor óptimo de cada uno de estos parámetros de escala depende de la función objetivo y del símplice inicial que se haya escogido. Por lo tanto, si se tiene una cierta experiencia en una clase de problemas en particular en el que se haya detectado que el método proporciona mejores resultados con otros factores de escala es razonable cambiar sus valores, de otro modo para simplificar el problema, es conveniente utilizar el doble y la mitad (Torczon1989). 
Por último, es importante en cuanto a la implementación del algoritmo definir un criterio de terminación. El algoritmo MDS está diseñado para que termine cuando la desviación típica en una cierta iteración se encuentre por debajo de un cierto valor, esto es:

$$
\sqrt{\sum_{i=0}^{n} \frac{\left(f\left(V_{i}^{k}\right)-\bar{f}\right)^{2}}{n}}<\varepsilon,
$$

donde $\overline{\mathrm{f}}$ es el valor medio de los valores de la función en los $n+1$ vértices del símplice y $\varepsilon$ representa el valor de la tolerancia prefijado. Este criterio funciona mientras el símplice no sea demasiado pequeño con respecto a la curvatura de la función hasta llegar al mínimo final. Este criterio puede llevar a que el método termine la optimización prematuramente debido a dos causas. En primer lugar, puede darse el caso que el símplice se encuentre encima de un mínimo local, lo que indica que el algoritmo ha sido capaz de encontrar las inmediaciones de un mínimo, sin embargo cualquiera de sus vértices constituiría un máximo local. En segundo lugar, se podría estar minimizando una función con poca curvatura, que puede producir que el algoritmo termine prematuramente. En ambos casos el valor de la función en cada uno de los puntos del símplice similar, sin embargo puede que los puntos que lo forman están todavía alejados.

Existen varias sugerencias para solucionar este problema, todas ellas encaminadas a aproximar los puntos del símplice antes de que pare el algoritmo. En el algoritmo de búsqueda multidireccional que se ha implementado se calcula la distancia máxima entre los puntos del símplice en función de su tamaño. En concreto el algoritmo comprueba:

$$
\frac{1}{\Delta} \max _{1 \leq \mathrm{i} \leq \mathrm{n}}\left\|\mathrm{V}_{\mathrm{i}}^{\mathrm{k}}-\mathrm{V}_{0}^{\mathrm{k}}\right\| \leq \varepsilon
$$

siendo $\Delta=\max \left(1,\left\|\mathrm{~V}^{0}{ }_{\mathrm{k}}\right\|\right)$, de esta manera se considera el tamaño relativo del símplice considerando la longitud del lado más largo adyacente al mejor vértice $\mathrm{V}^{0}{ }_{\mathrm{k}}$. Imponiendo la medida del cambio relativo entre los sucesivos valores $\mathrm{V}_{\mathrm{k}}^{0}$, se determina si el algoritmo ha terminado debido a un estancamiento del mismo, o porque ha alcanzado la convergencia. El valor de la tolerancia, $\varepsilon$, permitido en cada caso depende del número de dígitos significativos que se requieran (Torczon1989). Así, para el caso de requerirse p dígitos significativos la tolerancia, $\varepsilon$, tedrá un valor $\varepsilon=10^{-\mathrm{p}}$.

Además de fijar este criterio de terminación, y con el fin de asegurar que el algoritmo termina aunque no haya alcanzado una solución aceptable con respecto a la tolerancia impuesta, se ha fijado un número máximo de iteraciones de manera que la optimización termina al alcanzar este valor sea cual sea el resultado obtenido. 


\section{Capítulo 4}

\section{Aplicación a modelos termohidráulicos sencillos}

La elección de un método de optimización para resolver un problema determinado depende de las características del problema en sí. Por tanto, en este punto se debe decidir cuál de los métodos planteados en el capítulo anterior es el más eficiente al abordar el problema de búsqueda y optimización de parámetros termohidráulicos.

La comparación directa de ambas alternativas para resolver un problema complejo utilizando un código termohidráulico con el fin de escoger la mejor opción, no resulta, a priori, recomendable debido al tiempo de cálculo invertido, puesto que implica la ejecución del código un gran número de veces y con gran demanda de CPU. En efecto, según el escenario que se pretenda simular existen numerosos factores que hacen que el tiempo de cálculo necesario sea excesivo, como por ejemplo el tiempo de simulación, el paso máximo de tiempo de integración, los modelos que entran en la simulación etc. Por tanto, para la selección del método de optimización más idóneo, es preferible utilizar un caso simple con características similares a las que se plantean en el problema de búsqueda de parámetros para problemas más complejos mediante un código termohidráulico.

Además, la aplicación de la metodología a modelos termohidráulicos sencillos permitirá analizar y comprobar la viabilidad de la misma para tratar las incertidumbres asociadas con la resolución numérica, en base a la discretización espacial y temporal del problema, cuyas conclusiones son extrapolables a situaciones más complejas que se encuentran normalmente presentes en la simulación mediante códigos de estimación óptima. 
En general, esta posibilidad desaparece en la utilización de la mayoría de dichos códigos, o al menos su estudio se hace más difícil de abordar.

Como ejemplos representativos se han elegido la ecuación de la difusión y la del transporte unidimensionales, por ser modelos sencillos utilizados en numerosos campos de la ingeniería como en la difusión de aguas subterráneas (William1986), o del calor (Lenisc1995; Abou2000), y además, para estos modelos es posible calcular una solución analítica que tomaremos como solución de referencia.

En concreto, este capítulo se centra en el estudio de los parámetros del modelo de transferencia de calor en una dimensión considerando dos casos: en el primero se considera únicamente la difusión y en el segundo el proceso de convección-difusión.

Para que los problemas simplificados tengan las mismas características que los que se pueden plantear con un código termohidráulico de estimación óptima se realiza la búsqueda de parámetros a partir de los cálculos obtenidos utilizando métodos numéricos de resolución similares a los que se utilizan en estos códigos.

\subsection{Problema de la difusión de calor}

Para la aplicación de la metodología de estimación de parámetros a la ecuación de la difusión unidimensional, el problema concreto que se ha planteado consiste en la determinación de la difusividad de una barra unidimensional a partir de datos correspondientes a las temperaturas en distintos puntos de la barra en distintos instantes de tiempo. En concreto, en el instante inicial la barra se encuentra a $0^{\circ} \mathrm{C}$ y se aplica en ambos extremos una temperatura de $100^{\circ} \mathrm{C}$. Suponemos que la barra es de longitud unidad e imponemos las siguientes condiciones de contorno: $\mathrm{T}(0, \mathrm{t})=\mathrm{T}(1, \mathrm{t})=100$, junto con la condición inicial, $\mathrm{t}=0$, dada por la figura 4.1 .

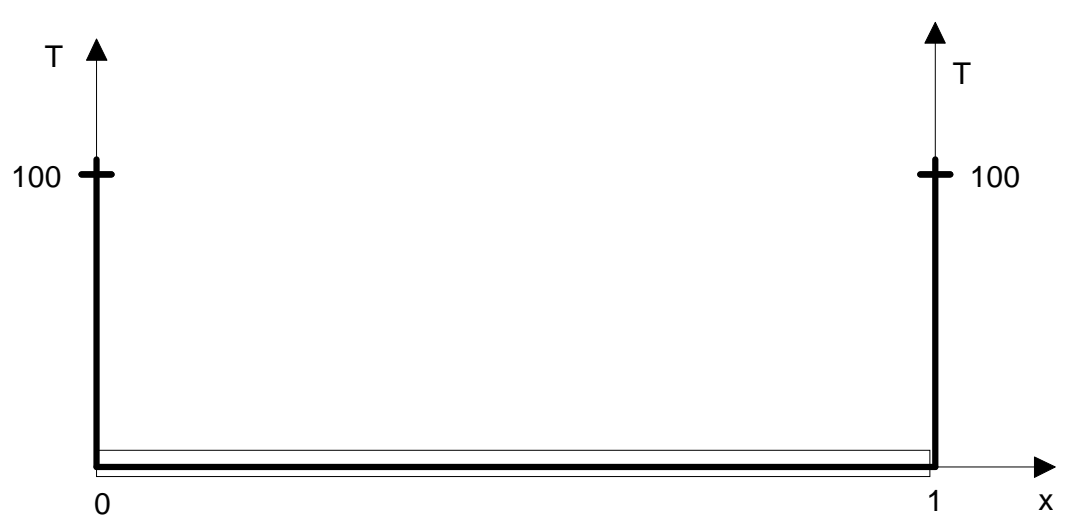

Figura 4.1: Condición inicial del problema de difusión del calor. 


\subsubsection{Ecuación de la difusión}

La variación de la temperatura con el tiempo en cada punto de la barra, se describe por medio de la ecuación unidimensional de la difusión que viene dada por:

$$
\frac{\partial \mathrm{T}}{\partial \mathrm{t}}-\alpha \frac{\partial^{2} \mathrm{~T}}{\partial \mathrm{x}^{2}}=0
$$

donde $\mathrm{T}$ es la temperatura en cada punto de la barra aislada en toda su longitud excepto por sus dos extremos, $\mathrm{t}$ es el tiempo, $\mathrm{x}$ la variable espacial, $\mathrm{y} \alpha$ es la difusividad térmica del material.

\subsubsection{Solución analítica}

Como ya se ha comentado anteriormente, los datos utilizados como referencia se obtendrán a partir de la solución analítica del problema. Para obtener esta solución se utiliza el método de separación de variables; esto es, se supone que es posible factorizar la función de temperatura de la siguiente manera

$T(x, t)=\tau(t) \cdot X(x)$

La ecuación (4.1) queda entonces de la forma

$$
\mathrm{X}(\mathrm{x}) \frac{\mathrm{d} \tau(t)}{\mathrm{dt}}=\alpha \cdot \tau(\mathrm{t}) \cdot \frac{\mathrm{d}^{2} \mathrm{X}(\mathrm{x})}{\mathrm{dx}^{2}}
$$

Se escoge $\mathrm{X}(\mathrm{x})$ tal que sea solución de una ecuación del tipo

$$
\frac{\mathrm{d}^{2} \mathrm{X}(\mathrm{x})}{\mathrm{dx}^{2}}=-\lambda \cdot \mathrm{X}(\mathrm{x})
$$

donde $\lambda$ es una constante a determinar. Sustituyendo (4.4) en la ecuación (4.1) queda

$$
\frac{\mathrm{d} \tau(t)}{\mathrm{dt}}=-\lambda \cdot \alpha \cdot \tau(\mathrm{t})
$$

cuya solución genérica es de la forma

$$
T(x, t)=C \cdot X(x) \cdot e^{-\lambda \cdot \alpha \cdot t}
$$


Tomaremos como conjunto fundamental de soluciones de la ecuación (4.4) el sistema trigonométrico $\{1, \operatorname{sen}(\mathrm{n} \pi \mathrm{x}), \cos (\mathrm{n} \pi \mathrm{x})\}$. De este modo, se tienen las posibilidades siguientes:

Si $\mathrm{X}(\mathrm{x})=1$

$$
\lambda=0
$$$$
\mathrm{T}=\mathrm{C}
$$

$\operatorname{Si} X(x)=\operatorname{sen}(n \pi x)$

$\lambda=(\mathrm{n} \pi)^{2}$

$T=C \operatorname{sen}(n \pi x) \exp \left(-\lambda n^{2} \pi^{2} t\right)$

Si $X(x)=\cos (n \pi x)$

$\lambda=(\mathrm{n} \pi)^{2}$

$\mathrm{T}=\mathrm{C} \cos (\mathrm{n} \pi \mathrm{x}) \exp \left(-\lambda \mathrm{n}^{2} \pi^{2} \mathrm{t}\right)$

Utilizando la notación de las series de Fourier, podemos escribir la solución general para $\mathrm{T}(\mathrm{x}, \mathrm{t})$ de la forma siguiente

$$
T(x, t)=\frac{a_{0}}{2}+\sum_{n=1}^{\infty} a_{n} \cdot \cos (n \pi x) \cdot \exp \left(-\alpha \pi^{2} n^{2} t\right)+\sum_{n=1}^{\infty} b_{n} \cdot \operatorname{sen}(n \pi x) \cdot \exp \left(-\alpha \pi^{2} n^{2} t\right)
$$

Los coeficientes $a_{0}, a_{n}, b_{n}$, se obtienen imponiendo que la temperatura satisfaga las condiciones iniciales que le han sido impuestas, las cuales vienen dadas por la función:

$\mathrm{T}(\mathrm{x}, 0)= \begin{cases}100 & \mathrm{x}=0 \\ 0 & 0<\mathrm{x}<1 \\ 100 & \mathrm{x}=1\end{cases}$

Por el teorema de Dirichlet, se tiene que $\mathrm{T}(\mathrm{x}, 0)$ es la función a la que converge la serie de Fourier de la extensión periódica, de periodo 2, a toda la recta real de una función $\mathrm{g}(\mathrm{x})$ cuya forma se muestra en la figura 4.2.

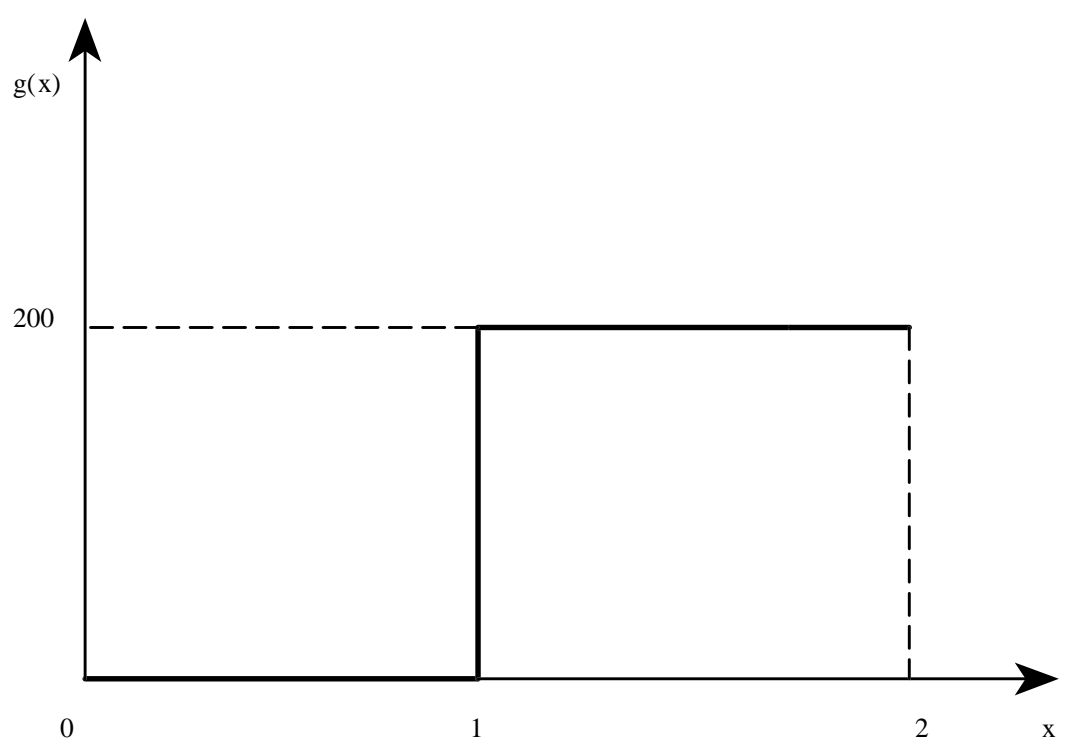

Figura 4.2: Función $\mathrm{g}(\mathrm{x})$ utilizada para el cálculo del desarrollo en serie de Fourier de las condiciones iniciales. 
Por otra parte, de la ecuación (4.7) en el instante $t=0$ tenemos:

$T(x, 0)=\frac{a_{0}}{2}+\sum_{n=1}^{\infty} a_{n} \cdot \cos (n \pi x)+\sum_{n=1}^{\infty} b_{n} \cdot \operatorname{sen}(n \pi x)$

así pues, se pueden calcular los valores de las constantes como:

$a_{0}=\int_{0}^{2} g(x) d x=\int_{1}^{2} 200 d x=200$

$a_{n}=\int_{0}^{2} g(x) \cos (n \pi x) d x=\int_{1}^{2} 200 \cos (n \pi x) d x=200\left[\frac{\operatorname{sen}(n \pi x)}{n \pi}\right]_{1}^{2}=0$

$b_{n}=\int_{0}^{2} g(x) \operatorname{sen}(n \pi x) d x=\int_{1}^{2} 200 \operatorname{sen}(n \pi x) d x=200\left[\frac{\cos (n \pi x)}{n \pi}\right]_{1}^{2}=-200 \frac{1}{n \pi}\left(1-(-1)^{n}\right)$

así el valor de las constantes $b_{n}$ será:

$\mathrm{b}_{\mathrm{n}}=\left\{\begin{array}{lll}0 & \mathrm{n}=2 \mathrm{k} & \mathrm{k}=1,2, \ldots \\ \frac{2}{(2 \mathrm{k}-1) \pi} & \mathrm{n}=2 \mathrm{k}-1 & \mathrm{k}=1,2, \ldots\end{array}\right.$

Sustituyendo los valores de las constantes en la ecuación (4.7) se obtiene la expresión de la temperatura en función del tiempo y de la variable espacial que satisface las condiciones iniciales anteriormente fijadas, que es de la forma

$$
\mathrm{T}(\mathrm{x}, \mathrm{t})=100-400 \sum_{\mathrm{k}=1}^{\infty} \frac{1}{(2 \mathrm{k}-1) \pi} \operatorname{sen}((2 \mathrm{k}-1) \pi \mathrm{x}) \exp \left(-\alpha(2 \mathrm{k}-1)^{2} \pi^{2} \mathrm{t}\right)
$$

\subsubsection{Solución numérica}

En las simulaciones de un proceso real utilizando un código termohidráulico se calcula el valor de las variables a partir de esquemas numéricos de cálculo, consiguiendo una solución aproximada a la real. El esquema del procedimiento de obtención de la solución numérica de una variable se muestra en la figura (4.3) (Fletcher1991). 


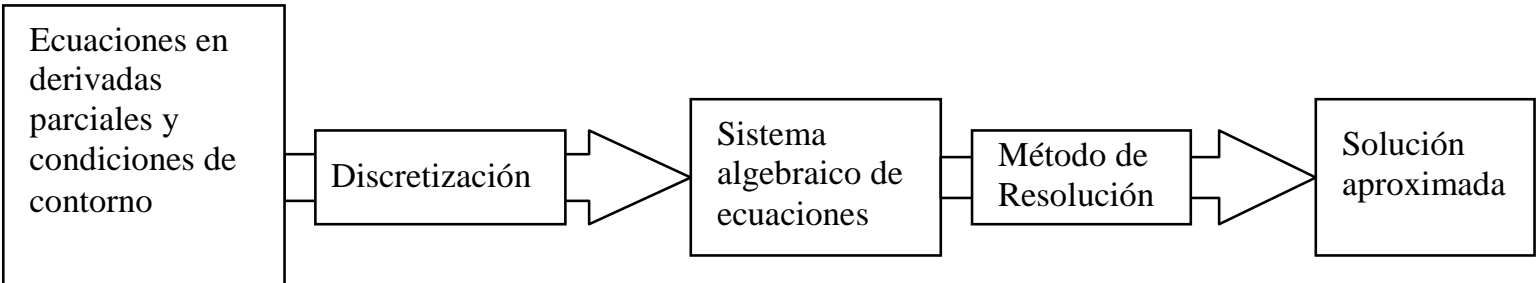

Figura 4.3: Proceso de obtención de la solución numérica.

Así pues, si observamos la figura 4.3, el proceso parte de las ecuaciones analíticas en derivadas parciales y de las condiciones de contorno del problema particular y mediante una discretización espacial y temporal se consigue definir el problema como un sistema algebraico de ecuaciones que aplicando un método de resolución conveniente nos proporciona el valor aproximado de la variable. Por tanto, para obtener una buena aproximación del valor de la variable se necesita una adecuada discretización espacial y temporal del problema y un método de resolución del sistema algebraico de ecuaciones capaz de calcular una solución aproximada a la real.

Una posible discretización espacial del problema de la ecuación de la difusión unidimensional empieza por aproximar las derivadas parciales como:

$$
\begin{aligned}
& \mathrm{T}(\mathrm{x}+\Delta \mathrm{x}, \mathrm{t})=\mathrm{T}(\mathrm{x}, \mathrm{t})+\frac{\partial \mathrm{T}(\mathrm{x}, \mathrm{t})}{\partial \mathrm{x}} \Delta \mathrm{x}+\frac{1}{2} \frac{\partial^{2} \mathrm{~T}(\mathrm{x}, \mathrm{t})}{\partial \mathrm{x}^{2}}(\Delta \mathrm{x})^{2}+\frac{1}{3 !} \frac{\partial^{3} \mathrm{~T}(\mathrm{x}, \mathrm{t})}{\partial \mathrm{x}^{3}}(\Delta \mathrm{x})^{3}+\mathrm{O}\left(\Delta \mathrm{x}^{4}\right) \\
& \mathrm{T}(\mathrm{x}-\Delta \mathrm{x}, \mathrm{t})=\mathrm{T}(\mathrm{x}, \mathrm{t})-\frac{\partial \mathrm{T}(\mathrm{x}, \mathrm{t})}{\partial \mathrm{x}} \Delta \mathrm{x}+\frac{1}{2} \frac{\partial^{2} \mathrm{~T}(\mathrm{x}, \mathrm{t})}{\partial \mathrm{x}^{2}}(\Delta \mathrm{x})^{2}-\frac{1}{3 !} \frac{\partial^{3} \mathrm{~T}(\mathrm{x}, \mathrm{t})}{\partial \mathrm{x}^{3}}(\Delta \mathrm{x})^{3}+\mathrm{O}\left(\Delta \mathrm{x}^{4}\right)
\end{aligned}
$$

donde $\mathrm{O}\left(\Delta \mathrm{x}^{4}\right)$ indica que el error de truncamiento de esta expresión es, al menos, proporcional a $\Delta \mathrm{x}^{4}$. Sumando las dos expresiones anteriores se llega a

$$
\mathrm{T}(\mathrm{x}+\Delta \mathrm{x}, \mathrm{t})+\mathrm{T}(\mathrm{x}-\Delta \mathrm{x}, \mathrm{t})=2 \mathrm{~T}(\mathrm{x}, \mathrm{t})+\frac{\partial^{2} \mathrm{~T}(\mathrm{x}, \mathrm{t})}{\partial \mathrm{x}^{2}}(\Delta \mathrm{x})^{2}+\mathrm{O}\left(\Delta \mathrm{x}^{4}\right)
$$

de donde se obtiene que:

$$
\frac{\partial^{2} T(x, t)}{\partial x^{2}}=\frac{1}{(\Delta x)^{2}}(T(x+\Delta x, t)-2 T(x, t)+T(x-\Delta x, t))+O\left(\Delta x^{2}\right)
$$


Por otra parte, para la discretización de la derivada temporal de la ecuación (4.1) los esquemas que se van a utilizar se ajustan a la fórmula general:

$\frac{\partial \mathrm{T}}{\partial \mathrm{t}} \approx(1-\beta) \mathrm{F}^{\mathrm{n}}+\beta \mathrm{F}^{\mathrm{n}+1}$

Siendo $F^{n}$ y $F^{n+1}$ el término $\alpha \frac{\partial^{2} T}{\partial x^{2}}$ evaluado en los tiempos $t=n y t=n+1$, respectivamente.

A su vez la derivada de la temperatura con respecto al tiempo puede aproximarse mediante la expresión:

$\frac{\partial \mathrm{T}}{\partial \mathrm{t}} \approx \frac{\mathrm{T}(\mathrm{x}, \mathrm{t}+\Delta \mathrm{t})-\mathrm{T}(\mathrm{x}, \mathrm{t})}{\Delta \mathrm{t}}$

La barra se divide en celdas o nodos como muestra la figura 4.4, y se introduce la notación $\mathrm{T}\left(\mathrm{x}_{\mathrm{i}}, \mathrm{t}_{\mathrm{n}}\right)=\mathrm{T}^{\mathrm{n}}{ }_{\mathrm{i}}, \mathrm{T}\left(\mathrm{x}_{\mathrm{i}}+\Delta \mathrm{x}, \mathrm{t}_{\mathrm{n}}\right)=\mathrm{T}^{\mathrm{n}}{ }_{\mathrm{i}+1}, \mathrm{~T}\left(\mathrm{x}_{\mathrm{i}}, \mathrm{t}_{\mathrm{n}}+\Delta \mathrm{t}\right)=\mathrm{T}^{\mathrm{n}+1}{ }_{\mathrm{i}}$.

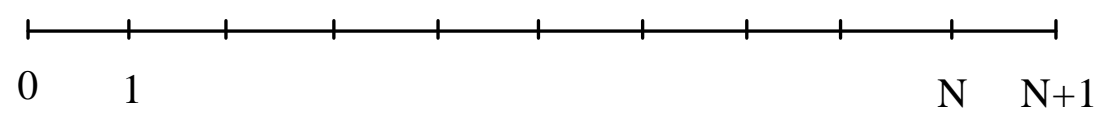

Figura 4.4: Discretización espacial de la barra.

Así, utilizando las aproximaciones dadas por las ecuaciones (4.16) y (4.18) y sustituyendo en la ecuación (4.17), se tiene que para un punto $\left(\mathrm{x}_{\mathrm{i}}, \mathrm{t}_{\mathrm{n}}\right)$ :

$\frac{\mathrm{T}_{\mathrm{i}}^{\mathrm{n}+1}-\mathrm{T}_{\mathrm{i}}^{\mathrm{n}}}{\Delta \mathrm{t}}=\alpha \frac{1}{(\Delta \mathrm{x})^{2}}\left((1-\beta)\left(\mathrm{T}_{\mathrm{i}+1}^{\mathrm{n}}-2 \mathrm{~T}_{\mathrm{i}}^{\mathrm{n}}+\mathrm{T}_{\mathrm{i}-1}^{\mathrm{n}}\right)+\beta\left(\mathrm{T}_{\mathrm{i}+1}^{\mathrm{n}+1}-2 \mathrm{~T}_{\mathrm{i}}^{\mathrm{n}+1}+\mathrm{T}_{\mathrm{i}-1}^{\mathrm{n}+1}\right)\right)$

que constituye el esquema general de cálculo utilizando una discretización mediante diferencias finitas. Escribiendo la ecuación (4.19) para los puntos $\mathrm{x}_{1}, \ldots, \mathrm{x}_{\mathrm{N}}$, se obtiene el sistema de ecuaciones que en notación matricial viene dado por: 


$$
\left[\begin{array}{ccccc}
\gamma_{1} & \gamma_{2} & 0 & \cdots & 0 \\
\gamma_{2} & \gamma_{1} & \gamma_{2} & \cdots & 0 \\
\vdots & & \ddots & & \vdots \\
0 & \cdots & \gamma_{2} & \gamma_{1} & \gamma_{2} \\
0 & \cdots & 0 & \gamma_{2} & \gamma_{1}
\end{array}\right]\left[\begin{array}{c}
\mathrm{T}_{1}^{\mathrm{n}+1} \\
\mathrm{~T}_{2}^{\mathrm{n}+1} \\
\vdots \\
\mathrm{T}_{\mathrm{N}-1}^{\mathrm{n}+1} \\
\mathrm{~T}_{\mathrm{N}}^{\mathrm{n}+1}
\end{array}\right]=\left[\begin{array}{c}
\mathrm{d}_{1} \\
\mathrm{~d}_{2} \\
\vdots \\
\mathrm{d}_{\mathrm{N}-1} \\
\mathrm{~d}_{\mathrm{N}}
\end{array}\right]
$$

Siendo,

$$
\begin{aligned}
& \gamma_{1}=\frac{1}{\Delta \mathrm{t}}+\frac{2 \alpha \beta}{(\Delta \mathrm{x})^{2}} \\
& \gamma_{2}=-\frac{\alpha \beta}{(\Delta \mathrm{x})^{2}} \\
& \mathrm{~d}_{1}=\frac{\alpha}{(\Delta \mathrm{x})^{2}} \mathrm{~T}_{0}^{\mathrm{n}}+\left[\frac{1}{\Delta \mathrm{t}}-\frac{2 \alpha(1-\beta)}{(\Delta \mathrm{x})^{2}}\right] \mathrm{T}_{1}^{\mathrm{n}}+\frac{\alpha(1-\beta)}{(\Delta \mathrm{x})^{2}} \mathrm{~T}_{2}^{\mathrm{n}} \\
& \mathrm{d}_{\mathrm{i}}=\frac{\alpha(1-\beta)}{(\Delta \mathrm{x})^{2}} \mathrm{~T}_{\mathrm{i}-1}^{\mathrm{n}}+\left[\frac{1}{\Delta \mathrm{t}}-\frac{2 \alpha(1-\beta)}{(\Delta \mathrm{x})^{2}}\right] \mathrm{T}_{\mathrm{i}}^{\mathrm{n}}+\frac{\alpha(1-\beta)}{(\Delta \mathrm{x})^{2}} \mathrm{~T}_{\mathrm{i}+1}^{\mathrm{n}} \quad \mathrm{i}=2, \ldots,(\mathrm{N}-1) \\
& \mathrm{d}_{\mathrm{N}}=\frac{\alpha(1-\beta)}{(\Delta \mathrm{x})^{2}} \mathrm{~T}_{\mathrm{N}-1}^{\mathrm{n}}+\left[\frac{1}{\Delta \mathrm{t}}-\frac{2 \alpha(1-\beta)}{(\Delta \mathrm{x})^{2}}\right] \mathrm{T}_{\mathrm{N}}^{\mathrm{n}}+\frac{\alpha}{(\Delta \mathrm{x})^{2}} \mathrm{~T}_{\mathrm{N}+1}^{\mathrm{n}}
\end{aligned}
$$

La matriz del sistema es una matriz tridiagonal y por tanto para su solución se utilizará un método de eliminación de Gauss adaptado a este tipo de sistemas (Press1992).

La constante $\beta$ mide el grado de implicitud del esquema, de este modo si $\beta=0$ la expresión resultante corresponde a un esquema explícito. En este caso no es necesaria la resolución del sistema y el valor de la temperatura en un nodo $\mathrm{j}$ y en un instante $\mathrm{n}+1$ depende de los valores de la temperatura en los nodos $j-1, j$ y j+1 del instante $n$, como se muestra en la figura 4.5. 


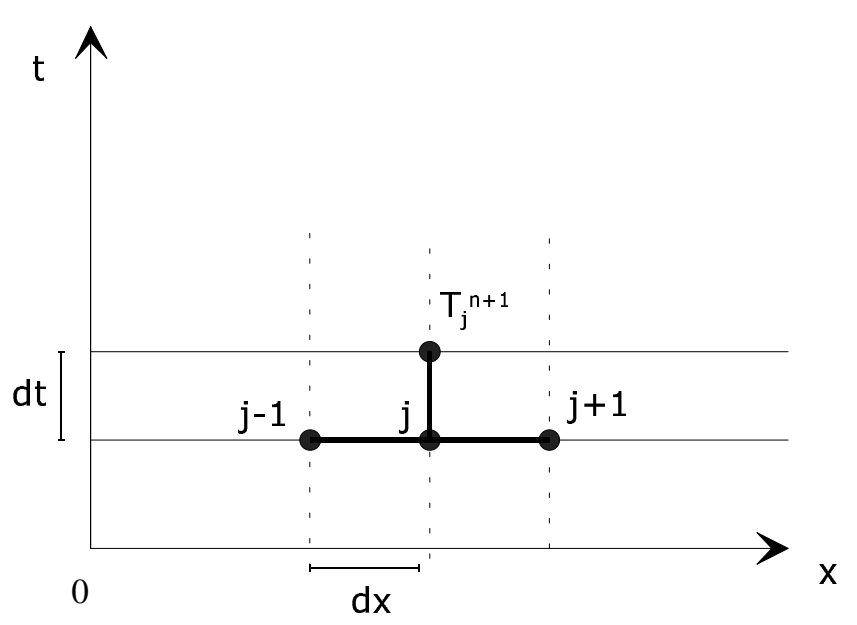

Figura 4.5: Esquema explícito.

En el caso de que $\beta=1$ la expresión algebraica corresponde al esquema completamente implícito y para $\beta=0.5$ al método de Crank-Nicolson (Fletcher1991). En estos dos casos se tiene que resolver el sistema de ecuaciones para obtener el valor de la temperatura en un nodo $\mathrm{j}$ y tiempo $\mathrm{n}+1$ partiendo de los nodos $\mathrm{j}-1, \mathrm{j}$ y $\mathrm{j}+1$ en el instante de tiempo $\mathrm{n}$ y los nodos de $\mathrm{j}-1 \mathrm{y} j+1$ en el instante de tiempo $n+1$ como se muestra en la figura 4.6.

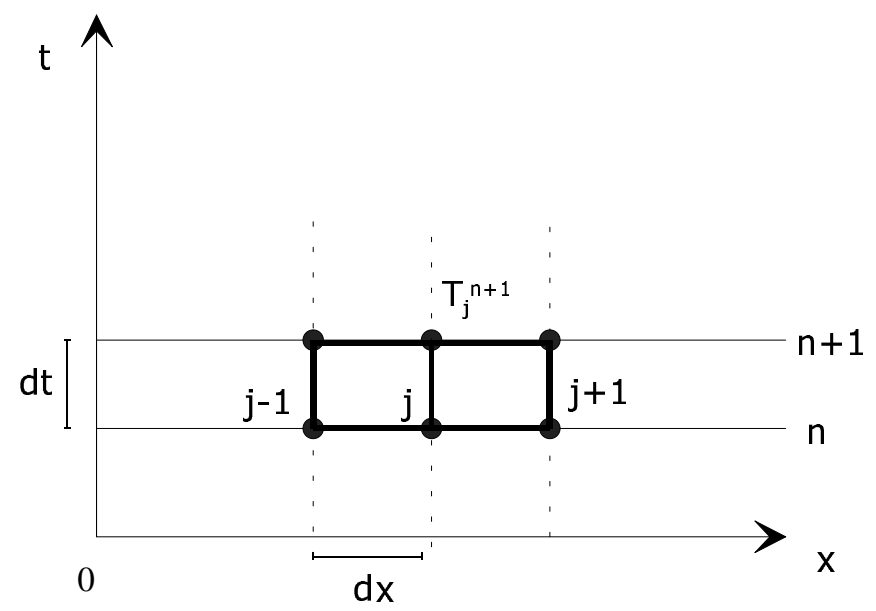

Figura 4.6: Esquema implícito.

Además, el valor de la constante $\beta$ determina la estabilidad del método de manera que si $0.5 \leqslant \beta \leqslant 1$ el esquema es incondicionalmente estable, mientras que si $0 \leqslant \beta \leqslant 0.5$ el método es estable siempre que verifique la expresión (Fletcher1991):

$\Delta \mathrm{t} \leq \frac{0.5(\Delta \mathrm{x})^{2}}{\alpha(1-2 \beta)}$ 
donde $\alpha$ es el valor de la difusividad, $\beta$ la constante de implicitud, $\Delta$ t el paso utilizado en la discretización temporal y $\Delta \mathrm{x}$ el paso de la discretización del espacio.

Como en este caso de aplicación se intenta estudiar si los métodos planteados en el capítulo anterior son capaces de encontrar el valor de la difusividad para un problema con una discretización determinada, se han considerado los intervalos de discretización fijos, tanto espacial como temporal, mientras que el parámetro $\alpha$ cambia de valor. Este planteamiento del problema puede ocasionar que para ciertos valores de $\alpha$ con significado físico la expresión dada por (4.26) no se verifique y el método numérico sea inestable, por lo que se ha desestimado el esquema de cálculo explícito, aunque el cálculo mediante este tipo de esquemas es mucho menos costoso desde el punto de vista computacional. En concreto se han realizado diversos cálculos en función del grado de implicitud que se desee, aplicando el proceso de búsqueda para el método de Crank-Nicolson $(\beta=0.5)$ que se encuentra en el límite de la estabilidad, y para el método implícito $(\beta=1)$ con la finalidad de determinar si este parámetro influye en el resultado de la búsqueda.

\subsubsection{Optimización del coeficiente de difusividad y estudios de sensibilidad}

Tal como se ha dicho anteriormente, el problema consiste en analizar las capacidades del algoritmo genético y del método de búsqueda multidireccional, explicados en el capítulo anterior, para la búsqueda del parámetro correspondiente al coeficiente de difusividad del código, $\alpha$, de la ecuación de la difusión, mediante dos aproximaciones numéricas, utilizando el método de Crank-Nicolson y el método implícito. Además, con los diferentes estudios de sensibilidad realizados se valorará la capacidad de la metodología en el cálculo de incertidumbres del cálculo numérico.

En este caso los valores obtenidos mediante la solución analítica actúan como datos experimentales que van a ser comparados con los cálculos aproximados de los métodos numéricos. Ambos métodos de búsqueda tomarán como función objetivo a minimizar la cuantificación del error cometido entre los valores de la solución analítica y de la numérica. Puesto que en el caso que nos ocupa se calculan valores de la temperatura en cada punto de la barra y para cada tiempo al final del transitorio el cálculo del error cometido por las aproximaciones numéricas consiste en calcular el error entre dos matrices, una con los valores numéricos y otra con los analíticos.

Con el objeto de cuantificar el error cometido en una simulación se han tomado los tres tipos de normas matriciales expuestos anteriormente (Burden1993): Norma 1, Norma $\propto$ y Norma de Frobenius. 
Para que el cálculo de la norma proporcione una cuantificación del error que se ha cometido en la simulación es necesario que los valores analíticos y numéricos que se comparen estén tomados en el mismo instante de tiempo y en la misma localización espacial. Sin embargo, en un problema real este no es el caso puesto que los datos experimentales se toman mediante detectores dispuestos a lo largo de la barra a una distancia que depende del número de detectores y de la longitud de la barra, y que en general no coinciden con el intervalo de discretización espacial elegido para el cálculo numérico, mucho más pequeño para conseguir resultados más precisos.

Lo mismo ocurre si se compara el intervalo de tiempo con el que un detector toma una muestra y el intervalo de tiempo de avance de la solución numérica. Por tanto, se debe contemplar una situación en la que de todos los cálculos realizados por el método de resolución numérica se deben elegir aquellos en los que existan valores analíticos con los que se puedan comparar. En la figura 4.7 se muestra un posible ejemplo en el que se han dispuesto cuatro detectores a lo largo de la barra, siendo la distancia entre detectores el doble del paso espacial de avance de la numérica, y el tiempo de toma de nuestra de cada uno de los detectores el doble del paso de avance temporal numérico.

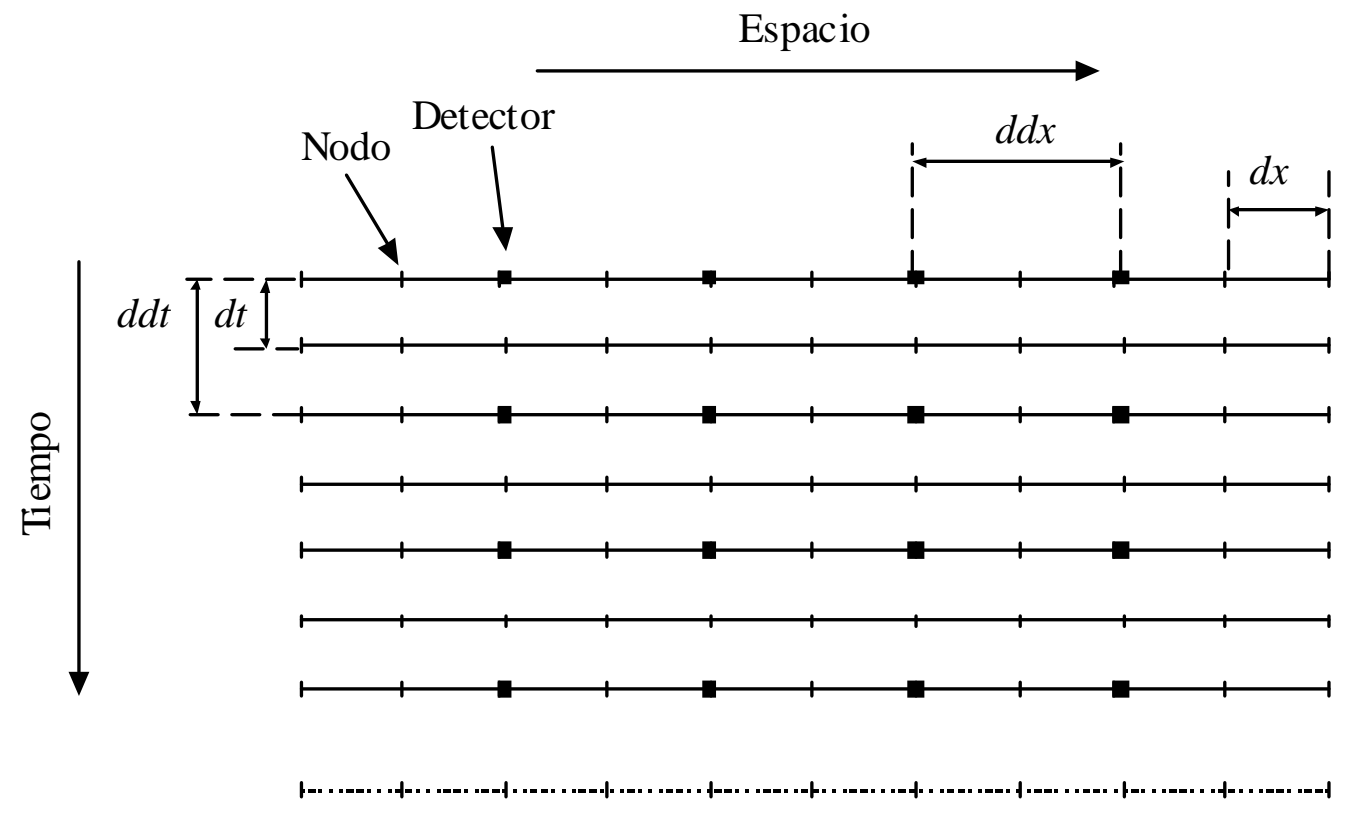

Figura 4.7: Ejemplo de disposición de detectores y discretización numérica.

Estas situaciones deben tenerse en cuenta en el análisis de los resultados obtenidos mediante cualquiera de los métodos de búsqueda que se plantean, por lo que es necesario la realización de análisis de sensibilidad de los resultados de la optimización en función de los diferentes factores tanto de la solución analítica como de la numérica que pueden afectar al resultado de la optimización. 
En la ecuación de la difusión de calor el parámetro a optimizar es la constante de difusividad, $\alpha$. En el caso de aplicación se ha tomado un valor de referencia de la difusividad de $\alpha=1.010^{-5} \mathrm{~m}^{2} / \mathrm{seg}$., y se han simulado $300 \mathrm{seg}$. de transitorio.

La solución analítica del problema viene dada por la ecuación (4.12), en la que aparece una serie infinita, de la que se consideran únicamente los 40 primeros términos para obtener una distribución de la temperatura a lo largo de la barra y en los distintos instantes de tiempo que debe compararse con los resultados aproximados de la solución numérica. Con este número finito de términos no se consigue reproducir la solución de forma suficientemente precisa en los instantes iniciales, por lo se han eliminado las temperaturas correspondientes a los tiempos menores de $30 \mathrm{seg}$.

Con este planteamiento, se han realizado diferentes estudios de sensibilidad variando los pasos temporales (DT) y el número de nodo espaciales (N) de la discretización numérica. Además, se han considerado distintas disposiciones espaciales de los detectores, así como diferentes tiempos de muestreo de los mismos. Para considerar la disposición espacial de los detectores se ha definido el parámetro KDTEC como la relación entre el número de detectores y el número de nodos espaciales. Así, KDTEC $=1$ indica que en cada nodo espacial hay un detector, KDTEC $=2$ indica que hay un detector cada dos nodos espaciales, esta última situación es la representada en la figura 4.7. Así, al aumentar el valor de este parámetro lo que se hace es disminuir el número de detectores a lo largo de la barra. Por último se ha utilizado el parámetro DDT que representa el periodo de muestreo de los detectores, puesto que en principio no tiene que ser el mismo que el paso de tiempo de la integración numérica, tal como se muestra en la figura 4.7.

En primer lugar se han realizado los cálculos utilizando como método de optimización el algoritmo MDS con las características que se indican en la tabla 4.1.

\begin{tabular}{ll}
\hline Características MDS & Valores \\
\hline Símplice inicial & 0.0 \\
& 0.01 \\
Paso contracción & 0.5 \\
Paso expansión & 2.0 \\
Iteraciones máximas & 100 \\
Evaluaciones máximas & 800 \\
Tolerancia & $1.010^{-5}$ \\
\hline
\end{tabular}

Tabla 4.1: Características del MDS para 1 parámetro.

El primero de los valores sobre los que se ha realizado el análisis de sensibilidad ha sido el número de detectores dispuestos en la barra manteniendo todos los demás factores 
invariables, y utilizando para la integración numérica un número de nodos $\mathrm{N}=63$ y un paso de tiempo DT=1.0, siendo el periodo de muestreo de los detectores DDT=DT=1.0.

Para los análisis de sensibilidad, los métodos numéricos expuestos anteriormente se han implementado utilizando el lenguaje $\mathrm{C}++$, y se han ejecutado en un ordenador Origin 2000 a $350 \mathrm{MHz}$ de Silicon Graphics.

En la tabla 4.2 se presentan los resultados obtenidos al variar KDTEC. En esta tabla se muestra el mejor valor para la difusividad encontrado por el algoritmo MDS, el error relativo cometido al utilizar las distintas normas matriciales, el número de evaluaciones de la función objetivo, y el tiempo de CPU invertido en cada optimización.

\begin{tabular}{llllllll}
\hline & & \multicolumn{3}{c}{ Crank-Nicolson: $\beta=0.5$} & \multicolumn{3}{c}{ Implícito: $\beta=1.0$} \\
\hline & & Norma Inf & Norma 1 & Frobenius & Norma Inf & Norma 1 & Frobenius \\
\hline KDETC=1 & valor alfa & $9.83310^{-6}$ & $9.98910^{-6}$ & $9.97510^{-6}$ & $9.82410^{-6}$ & $1.00010^{-5}$ & $9.99410^{-6}$ \\
$\mathrm{~N}=63$ & error & 0.00790 & 0.00211 & 0.00597 & 0.00910 & 0.00234 & 0.00732 \\
$\mathrm{DT}=1$ & evaluaciones & 122 & 134 & 98 & 122 & 122 & 94 \\
DDT=1 & tiempo (seg.) & 25.30 & 27.71 & 20.34 & 25.19 & 25.18 & 19.49 \\
\hline KDTEC=4 & valor alfa & $9.83710^{-6}$ & $9.99410^{-6}$ & $9.94910^{-6}$ & $9.83410^{-6}$ & $1.00210^{-6}$ & $9.97510^{-6}$ \\
$\mathrm{~N}=63$ & error & 0.01120 & 0.00631 & 0.01062 & 0.01259 & 0.00662 & 0.01244 \\
$\mathrm{DT}=1$ & evaluaciones & 134 & 94 & 106 & 126 & 94 & 90 \\
DDT $=1$ & tiempo (seg.) & 6.76 & 5.24 & 5.91 & 6.91 & 5.71 & 4.95 \\
\hline KDTEC $=16$ & valor alfa & $9.64810^{-6}$ & $9.61810^{-6}$ & $9.61810^{-6}$ & $9.58310^{-6}$ & $9.54610^{-6}$ & $9.54610^{-6}$ \\
$\mathrm{~N}=63$ & error & 0.02274 & 0.05202 & 0.05202 & 0.02729 & 0.06279 & 0.06279 \\
$\mathrm{DT}=1$ & evaluaciones & 134 & 94 & 94 & 146 & 98 & 98 \\
DDT $=1$ & tiempo (seg.) & 2.35 & 1.64 & 1.65 & 2.44 & 1.65 & 1.64 \\
\hline
\end{tabular}

Tabla 4.2: Análisis de la dependencia del número de detectores.

En esta tabla se observa que incluso utilizando un número muy pequeño de detectores el error relativo que se comente para cualquiera de las normas y esquemas utilizados es siempre menor del 7\%. Hay que hacer notar la rápida convergencia del problema hacia el óptimo, que se acelera a medida que se diminuye el número de detectores. Esto es debido a que disminuye el tamaño del problema, al disponer de menos detectores.

El segundo de los análisis de sensibilidad que se han realizado ha sido el cambio del número de nodos espaciales considerados para obtener la solución numérica, N. En este caso se ha considerado además diferentes números de detectores dispuestos a lo largo de la barra, y manteniendo fijo el paso temporal de la numérica y el periodo de muestreo de los detectores al valor DT=DDT=1.0. Los resultados obtenidos para el valor de la difusividad, el error cometido y el tiempo de CPU invertido en cada optimización de este estudio se presentan en la tabla 4.3. 
Si se comparan los resultados obtenidos en la tabla 4.3 en el caso de considerar el mismo número de nodos espaciales que detectores $($ KDTEC $=1)$ se observa que al disminuir el número de nodos el error aumenta considerablemente, de un $3 \%$ a un $12 \%$ de error máximo aproximadamente. Además, al disminuir el número de detectores en la barra el error mínimo que proporciona la optimización aumenta hasta alcanzar un 43\% aproximadamente en el caso de considerar únicamente dos detectores en toda la barra. No obstante el tiempo de cálculo también disminuye de forma importante.

\begin{tabular}{|c|c|c|c|c|c|c|}
\hline & \multicolumn{3}{|c|}{ Crank-Nicolson: $\beta=0.5$} & \multicolumn{3}{|c|}{ Implícito: $\beta=1.0$} \\
\hline & Norma Inf & Norma 1 & Frobenius & Norma Inf & Norma 1 & Frobenius \\
\hline \multicolumn{7}{|c|}{ KDTEC $=1$ DT $=$ DDT $=1$} \\
\hline $\mathrm{N}=31$ valor alfa & $9.46510^{-6}$ & $9.95610^{-6}$ & $9.86110^{-6}$ & $9.48410^{-6}$ & $9.96710^{-6}$ & $9.88010^{-6}$ \\
\hline error & 0.02828 & 0.01136 & 0.02714 & 0.02803 & 0.01148 & 0.02823 \\
\hline evaluaciones & 102 & 110 & 94 & 110 & 134 & 86 \\
\hline tiempo (seg.) & 10.47 & 11.24 & 9.65 & 11.23 & 14.65 & 8.84 \\
\hline $\mathrm{N}=15$ valor alfa & $8.34110^{-6}$ & $9.49810^{-6}$ & $8.80710^{-6}$ & $8.35210^{-6}$ & $9.51710^{-6}$ & $8.81410^{-6}$ \\
\hline error & 0.10098 & 0.08181 & 0.12557 & 0.10104 & 0.08169 & 0.12632 \\
\hline evaluaciones & 134 & 86 & 94 & 138 & 86 & 102 \\
\hline tiempo (seg.) & 6.70 & 4.32 & 4.72 & 6.86 & 4.31 & 5.08 \\
\hline \multicolumn{7}{|c|}{ KDTEC $=4$ DT $=$ DDT $=1$} \\
\hline \multirow[t]{4}{*}{$\mathrm{N}=31$} & $9.52610^{-6}$ & $9.54810^{-6}$ & $9.53910^{-6}$ & $9.51910^{-6}$ & $9.54310^{-6}$ & $9.53710^{-6}$ \\
\hline & 0.04207 & 0.06670 & 0.07029 & 0.04361 & 0.06927 & 0.07311 \\
\hline & 110 & 122 & 130 & 122 & 126 & 98 \\
\hline & 2.91 & 4.22 & 4.43 & 4.18 & 4.28 & 2.55 \\
\hline $\mathrm{N}=15$ valor alfa & $6.21110^{-6}$ & $5.76910^{-6}$ & $5.76910^{-6}$ & $6.18610^{-6}$ & $5.73910^{-6}$ & $5.73910^{-6}$ \\
\hline error & 0.16073 & 0.39856 & 0.39857 & 0.16203 & 0.40246 & 0.40247 \\
\hline evaluaciones & 142 & 118 & 98 & 146 & 106 & 106 \\
\hline tiempo (seg.) & 1.66 & 1.38 & 1.15 & 1.68 & 1.23 & 1.23 \\
\hline \multicolumn{7}{|c|}{ KDTEC $=16$ DT $=$ DDT $=1$} \\
\hline \multirow[t]{4}{*}{$\mathrm{N}=31$} & $6.56110^{-6}$ & $6.37710^{-6}$ & $6.37710^{-6}$ & $6.40810^{-6}$ & $6.23410^{-6}$ & $6.23410^{-6}$ \\
\hline & 0.19354 & 0.40116 & 0.40116 & 0.19355 & 0.41829 & 0.41829 \\
\hline & 82 & 126 & 126 & 82 & 114 & 114 \\
\hline & 0.6 & 0.91 & 0.92 & 0.57 & 0.79 & 0.79 \\
\hline
\end{tabular}

Tabla 4.3: Análisis de la dependencia del paso de integración espacial.

Por tanto, en este estudio se observa que el valor de la difusividad obtenido en la optimización depende fuertemente del número de nodos espaciales. De este modo, para cada valor del número de nodos utilizado se obtiene un valor óptimo de la difusividad distinto que ajusta mejor los valores experimentales. Este efecto tiene implicaciones importantes en la utilización de los códigos termohidráulicos, ya que al elegir una determinada nodalización en el diseño del modelo de una instalación puede ocurrir que los 
parámetros que mejor reproducen las medidas experimentales no sean los implementados en el código.

En cuanto a la dependencia del resultado de la optimización con el intervalo de tiempo en los que los detectores toman la medida de la temperatura, representado por la variable DDT, únicamente se ha realizado los estudios de sensibilidad para los casos en los que se han conseguido mejores resultados al variar KDTEC y $\mathrm{N}$, manteniendo DT $=1.0$. Los resultados de este estudio de sensibilidad se muestran en la tabla 4.4.

\begin{tabular}{|c|c|c|c|c|c|c|c|}
\hline & & \multicolumn{3}{|c|}{ Crank-Nicolson: $\beta=0.5$} & \multicolumn{3}{|c|}{ Implícito: $\beta=1.0$} \\
\hline & & Norma Inf & Norma 1 & Frobenius & Norma Inf & Norma 1 & Frobenius \\
\hline $\mathrm{N}=63$ & KDTEC $=1$ & & & & & & \\
\hline \multirow[t]{4}{*}{$\mathrm{DDT}=10$} & valor alfa & $9.83310^{-6}$ & $9.99410^{-6}$ & $9.97510^{-6}$ & $9.82410^{-6}$ & $1.00110^{-5}$ & $9.99410^{-6}$ \\
\hline & error & 0.0079 & 0.00218 & 0.00607 & 0.00910 & 0.00239 & 0.00742 \\
\hline & evaluaciones & 122 & 114 & 98 & 122 & 126 & 98 \\
\hline & tiempo (seg.) & 5.87 & 5.40 & 4.69 & 5.78 & 5.91 & 4.64 \\
\hline $\mathrm{N}=63$ & KDTEC $=4$ & & & & & & \\
\hline \multirow[t]{4}{*}{$\overline{\mathrm{DDT}}=10$} & valor alfa & $9.84110^{-6}$ & $9.99410^{-6}$ & $9.42710^{-6}$ & $9.839810^{-6}$ & $1.00210^{-5}$ & $9.97510^{-6}$ \\
\hline & error & 0.01094 & 0.00656 & 0.01076 & 0.01223 & 0.00691 & 0.01261 \\
\hline & evaluaciones & 126 & 94 & 86 & 126 & 106 & 86 \\
\hline & tiempo (seg.) & 4.13 & 4.07 & 2.79 & 4.03 & 4.39 & 2.74 \\
\hline $\mathrm{N}=31$ & $\mathrm{KDTEC}=1$ & & & & & & \\
\hline \multirow[t]{4}{*}{$\mathrm{DDT}=20$} & valor alfa & $9.46510^{-6}$ & $9.95610^{-6}$ & $9.86110^{-6}$ & $9.48410^{-6}$ & $9.98710^{-6}$ & $9.88310^{-6}$ \\
\hline & error & 0.02828 & 0.01173 & 0.02756 & 0.02803 & 0.01183 & 0.02875 \\
\hline & evaluaciones & 102 & 102 & 90 & 110 & 118 & 110 \\
\hline & tiempo (seg.) & 2.46 & 2.40 & 2.16 & 2.60 & 2.74 & 2.61 \\
\hline $\mathrm{N}=31$ & KDTEC $=4$ & & & & & & \\
\hline \multirow[t]{4}{*}{$\overline{\mathrm{DDT}}=20$} & valor alfa & $9.52610^{-6}$ & $9.55410^{-6}$ & $9.55410^{-6}$ & $9.52610^{-6}$ & $9.55410^{-6}$ & $9.55410^{-6}$ \\
\hline & error & 0.04205 & 0.06510 & 0.06884 & 0.04205 & 0.06520 & 0.06884 \\
\hline & evaluaciones & 126 & 134 & 122 & 126 & 134 & 122 \\
\hline & tiempo (seg.) & 2.06 & 2.19 & 2.00 & 2.06 & 2.19 & 2.00 \\
\hline
\end{tabular}

Tabla 4.4: Análisis del efecto del tiempo de muestreo.

Si se comparan los valores del error con los obtenidos en las tablas 4.2 y 4.3 para los mismos valores de $\mathrm{N}$ y KDTEC se observa que son prácticamente iguales, lo que indica que el resultado es poco sensible al aumento del periodo de muestreo de los detectores. Además, al aumentar el periodo de muestreo disminuye el tiempo necesario para obtener el óptimo, por ejemplo si consideramos el caso en que $\mathrm{N}=63$ y KDTEC $=1$ el tiempo de cálculo se reduce para todas las normas y en los dos esquemas de cálculo considerados, mientras que los valores obtenidos para el error son prácticamente iguales. 
El último de los casos realizados consiste en el cambio de paso temporal de integración de la solución numérica, DT, aumentándolo al doble. En este caso concreto se ha tomado en consideración los resultados obtenidos en todos los demás análisis, considerando únicamente aquellas combinaciones de valores para los que el aumento del paso temporal de integración pudiera suponer una variación en los resultados de la optimización. Como se ha observado que al ampliar el periodo de muestreo se reduce el tiempo de cálculo obteniendo buenos resultados, se han realizado las optimizaciones considerando un DDT=10 seg en todos los cálculos, variando el número de nodos con los que se ha obtenido la solución numérica con una relación entre nodos y detectores KDTEC=4. Los valores obtenidos en estas últimas comprobaciones se presentan en la tabla 4.5.

\begin{tabular}{|c|c|c|c|c|c|c|c|}
\hline & & \multicolumn{3}{|c|}{ Crank-Nicolson: $\beta=0.5$} & \multicolumn{3}{|c|}{ Implícito: $\beta=1.0$} \\
\hline & & Norma Inf & Norma 1 & Frobenius & Norma Inf & Norma 1 & Frobenius \\
\hline $\mathrm{N}=63$ & $\mathrm{DDT}=10$ & KDTEC $=4$ & & & & & \\
\hline \multirow[t]{4}{*}{$\overline{\mathrm{DT}}=2$} & valor alfa & $9.83410^{-6}$ & $9.99410^{-6}$ & $9.97510^{-6}$ & $9.88110^{-6}$ & $1.00410^{-5}$ & $1.0010^{-5}$ \\
\hline & error & 0.00786 & 0.00217 & 0.00605 & 0.01061 & 0.00303 & 0.00885 \\
\hline & evaluaciones & 118 & 114 & 98 & 106 & 126 & 90 \\
\hline & tiempo (seg.) & 2.82 & 2.72 & 2.36 & 2.51 & 2.97 & 2.31 \\
\hline $\mathrm{N}=31$ & $\mathrm{DDT}=10$ & KDTEC $=4$ & & & & & \\
\hline \multirow[t]{4}{*}{$\mathrm{DT}=2$} & valor alfa & $9.52610^{-6}$ & $9.55410^{-6}$ & $9.55410^{-6}$ & $9.51310^{-6}$ & $9.54310^{-6}$ & $9.54310^{-6}$ \\
\hline & error & 0.04205 & 0.06521 & 0.06885 & 0.04513 & 0.07019 & 0.07435 \\
\hline & evaluaciones & 130 & 106 & 102 & 130 & 114 & 126 \\
\hline & tiempo (seg.) & 0.54 & 0.45 & 0.43 & 0.53 & 0.46 & 0.51 \\
\hline
\end{tabular}

Tabla 4.5: Análisis en función del paso temporal de integración.

En la tabla anterior se observa que el error cometido al duplicar el paso de integración temporal se encuentra siempre por debajo de un $1 \%$ en el caso de considerar 63 nodos en la numérica para cualquiera de las normas y esquemas de cálculo considerados. En este caso al variar el número de nodos espaciales también aumenta el error cometido en el punto óptimo.

Así, se observa que la variable que más afecta al resultado de la optimización es el número de nodos de la numérica. Además, en todos los estudios de sensibilidad se observa que bajo unos parámetros para la numérica y analítica determinados, el resultado de la optimización presenta poca dependencia de la norma que se utilice para cuantificar el error cometido en la simulación. 
El mismo problema de optimización se ha resuelto utilizando un algoritmo genético de estado estacionario (AGEE). Sin embargo en este caso no se han considerado tantos análisis de sensibilidad, sino que el estudio se ha centrado en los parámetros cuya variación afectan de forma apreciable al resultado de la optimización. Los parámetros del AGEE escogidos para realizar los diferentes análisis de sensibilidad se muestran en la tabla 4.6, los cuales se encuentran explicados con detalle en el capítulo anterior.

\begin{tabular}{ll}
\hline Parámetro AGEE & \\
\hline Codificación & Real \\
Tamaño población & 50 \\
Probabilidad de cruce & 0.6 \\
Probabilidad de mutación & 0.99 \\
Número de máximo de generaciones & 500 \\
Porcentaje de convergencia & 0.25 \\
Porcentaje de reemplazamiento & 0.9 \\
Método de selección & GATournamentSelector \\
Método de cruce & OnePointCrossover \\
Método de mutación & FlipMutator \\
Método de escalado & GAPowerLawScaling \\
Método de terminación & UponConvergenceUPV \\
\hline
\end{tabular}

Tabla 4.6: Características del AGEE para 1 parámetro.

La población inicial se ha tomado 50 individuos de los cuales se han inicializado dos individuos a los valores que definen el símplice inicial del algoritmo MDS, presentados en la tabla 4.1, mientras que la población restante se elige de manera aleatoria. Con esta inicialización se pretende que el algoritmo al iniciar la búsqueda tenga la misma información de la que parte el MDS. Se ha utilizado la codificación real para representar los individuos de la población, los cuales están formados por un solo gen que representa la difusividad.

En cuanto al criterio de terminación, se ha elegido un valor de 500 como número máximo de generaciones a realizar, aunque no se haya alcanzado la convergencia, un porcentaje de convergencia de la población del 0.65 y un valor para la convergencia genética de 0.99 .

Con todas estas características se han desarrollado los estudios de sensibilidad variando únicamente los parámetros que para el MDS han demostrado una influencia clara en el resultado. Es decir, se han variado el número de nodos de la numérica, $\mathrm{N}$, y la disposición de los detectores, KDTEC, para una simulación de TF=300 seg. en la que se consideran a partir de un tiempo TINI=30 seg., utilizando NTER=40 en la solución analítica y considerando el paso de integración de la numérica y el periodo de detección iguales y de 
valor $\mathrm{DT}=\mathrm{DDT}=1.0$. En la tabla 4.7 se muestran los resultados obtenidos en los análisis de sensibilidad utilizando el AGEE.

\begin{tabular}{|c|c|c|c|c|c|c|c|}
\hline & & \multicolumn{3}{|c|}{ Crank-Nicolson: $\beta=0.5$} & \multicolumn{3}{|c|}{ Implícito: $\beta=1.0$} \\
\hline & & Norma Inf & Norma 1 & Frobenius & Norma Inf & Norma 1 & Frobenius \\
\hline \multicolumn{8}{|c|}{ KDTEC $=1 \mathrm{DT}=1.0 \mathrm{DDT}=1.0$} \\
\hline \multirow[t]{4}{*}{$\mathrm{N}=63$} & valor alfa & $1.00310^{-5}$ & $9.89810^{-6}$ & $1.00610^{-5}$ & $1.65710^{-6}$ & $2.16310^{-5}$ & $9.73910^{-6}$ \\
\hline & error & 0.00891 & 0.00354 & 0.00856 & 0.6656 & 0.2208 & 0.01531 \\
\hline & generaciones & 199 & 156 & 176 & 35 & 37 & 169 \\
\hline & tiempo (seg) & 814.94 & 652.66 & 718.66 & 144.84 & 156.38 & 677.9 \\
\hline \multirow[t]{4}{*}{$\mathrm{N}=31$} & valor alfa & $8.41210^{-6}$ & $2.21110^{-5}$ & $1.01810^{-5}$ & $9.07010^{-6}$ & $1.55010^{-5}$ & $1.11410^{-5}$ \\
\hline & error & 0.10225 & 0.3177 & 0.03678 & 0.11421 & 0.17761 & 0.10091 \\
\hline & generaciones & 137 & 24 & 176 & 95 & 43 & 103 \\
\hline & tiempo (seg.) & 136.39 & 51.92 & 356.71 & 94.36 & 89.45 & 205.38 \\
\hline \multirow[t]{4}{*}{$\mathrm{N}=15$} & valor alfa & $1.06710^{-5}$ & $1.85210^{-5}$ & $1.71310^{-6}$ & $8.795410^{-6}$ & $8.63610^{-6}$ & $7.19310^{-6}$ \\
\hline & error & 0.50853 & 0.56336 & 0.76469 & 0.10907 & 0.10021 & 0.18731 \\
\hline & generaciones & 57 & 11 & 63 & 111 & 103 & 81 \\
\hline & tiempo (seg.) & 57.29 & 12.16 & 64.27 & 108.99 & 104.16 & 79.48 \\
\hline \multicolumn{8}{|c|}{ KDTEC $=4$ DT $=1.0$ DDT $=1.0$} \\
\hline \multirow[t]{4}{*}{$\mathrm{N}=63$} & valor alfa & $7.19410^{-6}$ & $1.03510^{-5}$ & $8.02110^{-6}$ & $1.43110^{-5}$ & $9.01810^{-6}$ & $9.16910^{-6}$ \\
\hline & error & 0.25442 & 0.03302 & 0.20144 & 0.34383 & 0.07907 & 0.07724 \\
\hline & generaciones & 72 & 149 & 102 & 86 & 171 & 199 \\
\hline & tiempo (seg.) & 119.01 & 247.28 & 167.36 & 147.89 & 295.44 & 339.06 \\
\hline \multirow[t]{4}{*}{$\mathrm{NX}=31$} & valor alfa & $4.039510^{-6}$ & $2.77510^{-6}$ & $9.98310^{-6}$ & $6.20110^{-6}$ & $1.19910^{-5}$ & $1.29110^{-5}$ \\
\hline & error & 0.75609 & 0.95304 & 0.13948 & 0.16498 & 0.56800 & 0.7900 \\
\hline & generaciones & 138 & 81 & 153 & 193 & 69 & 66 \\
\hline & tiempo (seg.) & 52.65 & 65.92 & 122.04 & 76.38 & 58.44 & 55.33 \\
\hline \multirow[t]{4}{*}{$\mathrm{Nx}=15$} & valor alfa & $7.063110^{-6}$ & $2.77710^{-6}$ & $5.33410^{-6}$ & $5.43210^{-6}$ & $5.50310^{-6}$ & $5.94910^{-6}$ \\
\hline & error & 0.3891 & 0.96371 & 0.44003 & 0.46479 & 0.41586 & 0.408189 \\
\hline & generaciones & 137 & 126 & 159 & 104 & 158 & 142 \\
\hline & tiempo (seg.) & 52.21 & 48.03 & 60.07 & 41.57 & 62.64 & 56.02 \\
\hline
\end{tabular}

Tabla 4.7: Análisis de sensibilidad para el AGEE de 1 parámetro.

Observando la tabla 4.7 se pone de manifiesto una gran disparidad en los resultados alcanzados con el AGEE que se puede explicar por una convergencia prematura independientemente del esquema de cálculo utilizado, como puede observarse en el caso de $\mathrm{N}=63$ y KDTEC $=1$ para la Norma Infinito y la Norma 1 en el esquema implícito, o en el caso de $\mathrm{N}=15$ y KDTEC=1 para la Norma 1 en el esquema de Crank-Nicolson.

Se estima que el problema de la convergencia prematura se puede evitar cambiando el criterio de terminación y obligando al algoritmo genético a realizar un cierto número de generaciones para el que se considera que ya no hay opción a que se dé este problema. Así, 
se comprueba que para el esquema de cálculo implícito con 63 nodos y un detector por cada nodo los resultados que aparecen en la tabla 4.7 presentan una mejora sustancial si se exige que se realicen 200 generaciones, tal como puede observarse en la tabla 4.8 en cualquiera de las tres normas elegidas para el cálculo del error.

\begin{tabular}{|c|c|c|c|c|}
\hline & & \multicolumn{3}{|c|}{ Implícito: $\beta=1.0$} \\
\hline & & Norma Inf & Norma 1 & Frobenius \\
\hline \multicolumn{5}{|c|}{ KDTEC $=1$} \\
\hline \multirow[t]{4}{*}{$\overline{\mathrm{N}=63}$} & valor alfa & $1.10110^{-5}$ & $1.09910^{-5}$ & $9.86010^{-6}$ \\
\hline & error & 0.05749 & 0.02762 & 0.01015 \\
\hline & generaciones & 200 & 200 & 200 \\
\hline & tiempo (seg.) & 802.55 & 820.37 & 821.44 \\
\hline
\end{tabular}

Tabla 4.8: Resultados a 200 generaciones.

Atendiendo a los resultados anteriores, se observa que en cualquier caso el AGEE es capaz de obtener valores de error del mismo orden de magnitud que los que alcanza el algoritmo MDS en estas condiciones, sin embargo el tiempo de CPU invertido en la optimización mediante el AGEE es extremadamente grande comparado con el que necesita el MDS. Como ejemplo, considerando los tiempos invertidos en los cálculos más largos, que corresponden a $\mathrm{N}=63$ y $\mathrm{KDTEC}=1$, el MDS necesita unos $25 \mathrm{seg}$. aproximadamente para alcanzar un error que se sitúa sobre el 1 o 2\%, mientras que el AGEE invierte unos 800 seg. en conseguir un error del mismo orden de magnitud.

Este hecho puede generalizarse a cualquiera de los casos de sensibilidad realizados y es debido a la manera de proceder diferente de ambos métodos de optimización. Así, mientras que el MDS parte de un símplice inicial, que en este caso está compuesto por dos puntos, el AGEE necesita una población inicial que para garantizar el buen funcionamiento del algoritmo debe ser como mínimo de 50 individuos (Goldberg1989). Además, en el proceso evolutivo el MDS realiza como máximo 3 cálculos mientras que para el AGEE el número de cálculos depende de la probabilidad de reemplazamiento especificada por el usuario, que en este caso concreto es muy elevada debido a que al tener únicamente un gen es necesario generar un número elevado de individuos nuevos para garantizar la mejora en la optimización y evitar la convergencia prematura.

Finalmente indicar que del mismo modo que ocurre en los análisis de sensibilidad realizados con el algoritmo MDS los resultados proporcionados por el AGEE presentan una dependencia clara del número de nodos espaciales que se consideren en la solución numérica, con lo que con ambos métodos se llegan a las mismas conclusiones. 


\subsection{Problema de la convección-difusión del calor}

Otro problema más complicado que el tratado en la sección anterior consiste en la estimación de parámetros para la ecuación de la convección-difusión unidimensional.

El problema particular consiste en encontrar una solución para la temperatura en una región de longitud $\mathrm{L}$, con las condiciones de contorno $\mathrm{T}(0, \mathrm{t})=1 ; \mathrm{T}(\mathrm{L}, \mathrm{t})=0$ y la condición inicial que se muestran en la figura 4.8 .

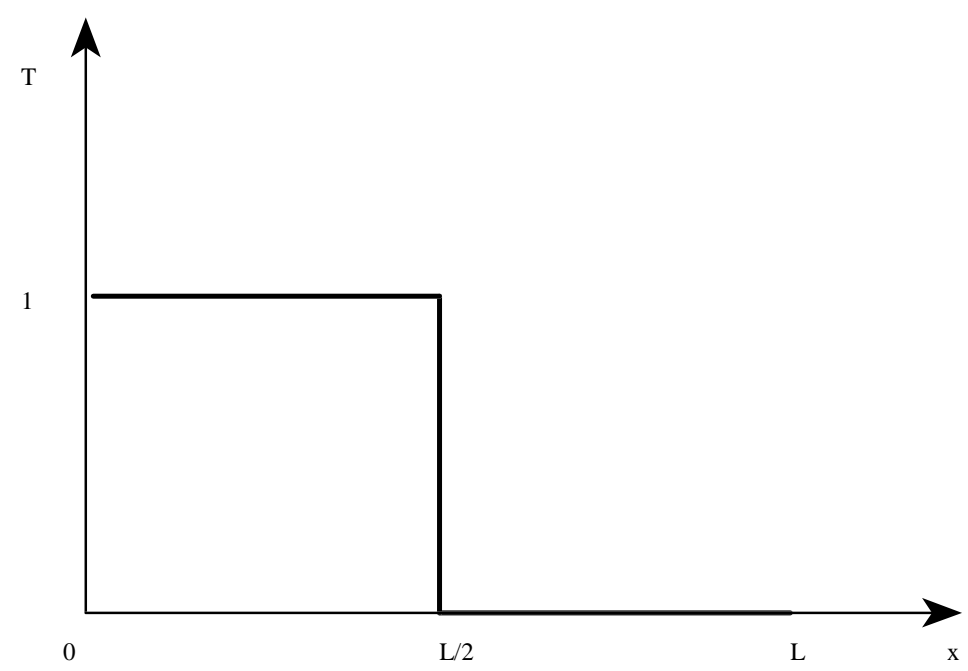

Figura 4.8: Condición inicial para el problema de convección-difusión.

O sea,

$\mathrm{T}(\mathrm{x}, 0)=1-\mathrm{H}\left(\mathrm{x}-\frac{\mathrm{L}}{2}\right)$,

donde tomaremos $\mathrm{L}=1 \mathrm{y} \mathrm{H}(\mathrm{x})$ es una función de la forma

$H(x)= \begin{cases}1 & x \geq 0 \\ 0 & x<0\end{cases}$

Este problema tiene una solución analítica, que utilizaremos como solución de referencia en el problema de estimación de parámetros y que se deduce a continuación. 


\subsubsection{Ecuación de convección-difusión unidimensional}

Esta ecuación modeliza fenómenos como el comportamiento del avance con una velocidad $\mathrm{v}$ de un frente de temperatura cuando se mezclan dos fluidos que inicialmente se encuentran a temperaturas diferentes. La temperatura en función del tiempo y la posición se rige por

$\frac{\partial \mathrm{T}(\mathrm{x}, \mathrm{t})}{\partial \mathrm{t}}+\mathrm{v} \frac{\partial \mathrm{T}(\mathrm{x}, \mathrm{t})}{\partial \mathrm{x}}=\alpha \frac{\partial^{2} \mathrm{~T}(\mathrm{x}, \mathrm{t})}{\partial \mathrm{x}^{2}}$,

donde $\mathrm{v}$ es la velocidad de convección, $\alpha$ es la difusividad térmica y $\mathrm{T}(\mathrm{x}, \mathrm{t})$ es la temperatura.

\subsubsection{Solución analítica}

Para obtener la solución analítica de este problema en primer lugar se elimina la parte convectiva, utilizando el cambio de variable:

$\mathrm{T}(\mathrm{x}, \mathrm{t})=\exp \left(\frac{\mathrm{v}}{2 \alpha}\left(x-\frac{\mathrm{t}}{2}\right)\right) \mathrm{w}(\mathrm{x}, \mathrm{t})$

con lo que se llega a una ecuación para w(x,t) de la forma

$\frac{\partial \mathrm{w}(\mathrm{x}, \mathrm{t})}{\partial \mathrm{t}}=\alpha \frac{\partial^{2} \mathrm{w}(\mathrm{x}, \mathrm{t})}{\partial \mathrm{x}^{2}}$

expresándose las condiciones de contorno y la condición inicial de la siguiente manera

$$
\begin{aligned}
& \mathrm{w}(0, \mathrm{t})=\exp \left(\frac{\mathrm{v}^{2} \mathrm{t}}{4 \alpha}\right), \\
& \mathrm{w}(\mathrm{L}, \mathrm{t})=0 \\
& \mathrm{w}(\mathrm{x}, 0)=\exp \left(\frac{-\mathrm{v} \mathrm{x}}{2 \alpha}\right)\left(1-\mathrm{H}\left(\mathrm{x}-\frac{\mathrm{L}}{2}\right)\right) .
\end{aligned}
$$

Con este cambio se llega a un problema con condiciones de contorno no homogéneas. Para resolver este nuevo problema se utiliza el cambio

$$
\mathrm{w}(\mathrm{x}, \mathrm{t})=\left(1-\mathrm{H}\left(\mathrm{x}-\frac{\mathrm{L}}{2}\right)\right) \exp \left(\frac{\mathrm{v}^{2} \mathrm{t}}{4 \mathrm{a}}\right)+\mathrm{u}(\mathrm{x}, \mathrm{t})
$$


con lo que la ecuación (4.29) queda para la función u(x,t) de la forma:

$$
\frac{\partial \mathrm{u}(\mathrm{x}, \mathrm{t})}{\partial \mathrm{t}}-\alpha \frac{\partial^{2} \mathrm{u}(\mathrm{x}, \mathrm{t})}{\partial \mathrm{x}^{2}}=-\frac{\mathrm{v}^{2}}{4 \alpha}\left(1-\frac{\mathrm{x}}{\mathrm{L}}\right) \exp \left(\frac{\mathrm{v}^{2} \mathrm{t}}{4 \alpha}\right)
$$

siendo en este caso las nuevas condiciones de contorno y la condición inicial

$$
\begin{aligned}
& u(0, t)=u(L, t)=0 \\
& u(x, 0)=\exp \left(-\frac{v x}{2 \alpha}\right)\left(1-H\left(x-\frac{L}{2}\right)\right)-\left(1-\frac{x}{L}\right) .
\end{aligned}
$$

Para este problema se buscan soluciones del tipo

$u(x, t)=\widetilde{u}(x, t)+z(x, t)$

donde $\widetilde{\mathrm{u}}(\mathrm{x}, \mathrm{t})$ es solución del problema homogéneo

$$
\frac{\partial \widetilde{\mathrm{u}}(\mathrm{x}, \mathrm{t})}{\partial \mathrm{t}}=\alpha \frac{\partial^{2} \widetilde{\mathrm{u}}(\mathrm{x}, \mathrm{t})}{\partial \mathrm{x}^{2}}
$$

con las condiciones

$$
\begin{aligned}
& \widetilde{\mathrm{u}}(0, \mathrm{t})=\widetilde{\mathrm{u}}(\mathrm{L}, \mathrm{t})=0 \\
& \widetilde{\mathrm{u}}(\mathrm{x}, 0)=\exp \left(-\frac{\mathrm{v} x}{2 a ́}\right)\left(1-\mathrm{H}\left(\mathrm{x}-\frac{\mathrm{L}}{2}\right)\right)-\left(1-\frac{\mathrm{x}}{\mathrm{L}}\right) .
\end{aligned}
$$

La solución general al problema es de la forma

$$
\widetilde{u}(x, t)=\sum_{n=1}^{\infty} c_{n} \operatorname{sen}\left(\frac{n \pi}{L} x\right) \exp \left(-\alpha\left(\frac{n \pi}{L}\right)^{2} t\right)
$$

y por tanto se ha de cumplir la condición inicial

$$
\exp \left(-\frac{\mathrm{vx}}{2 \alpha}\right)\left(1-\mathrm{H}\left(1-\frac{\mathrm{x}}{\mathrm{L}}\right)\right)=\sum_{n=1}^{\infty} \mathrm{c}_{\mathrm{n}} \operatorname{sen}\left(\frac{\mathrm{n} \pi \mathrm{x}}{\mathrm{L}}\right)
$$

Utilizando que 


$$
\frac{2}{L} \int_{0}^{L} \operatorname{sen}\left(\frac{n \pi x}{L}\right) \operatorname{sen}\left(\frac{m \pi x}{L}\right) d x=\delta_{n, m},
$$

se llega a que

$$
\begin{aligned}
& c_{n}=\frac{2}{\frac{L v^{2}}{4 \alpha^{2}}+\frac{n^{2} \pi^{2}}{L}}\left(-\frac{n \pi}{L} \exp \left(-\frac{v L}{4 \alpha}\right) \cos \left(\frac{n \pi}{2}\right)-\frac{v}{2 \alpha} \exp \left(-\frac{v L}{4 \alpha}\right) \operatorname{sen}\left(\frac{n \pi}{2}\right)+\frac{n \pi}{L}\right)+ \\
& \frac{2}{n^{2} \pi^{2}} \operatorname{sen}(n \pi)-\frac{2}{n \pi} .
\end{aligned}
$$

A partir de la ecuación (4.32) se obtiene:

$$
\frac{\partial \mathrm{z}}{\partial \mathrm{t}}-\alpha \frac{\partial^{2} \mathrm{z}}{\partial \mathrm{x}^{2}}=-\frac{\mathrm{v}^{2}}{4 \alpha}\left(1-\frac{\mathrm{x}}{\mathrm{L}}\right) \exp \left(\frac{\mathrm{v}^{2} \mathrm{t}}{4 \alpha}\right)
$$

suponemos que

$$
z(x, t)=\sum_{n=1}^{\infty} z_{n}(t) \operatorname{sen}\left(\frac{n \pi x}{L}\right),
$$

y teniendo en cuenta que

$$
1-\frac{\mathrm{x}}{\mathrm{L}}=\sum_{m=1}^{\infty} \frac{2}{\mathrm{~m} \pi} \operatorname{sen}\left(\frac{\mathrm{m} \pi \mathrm{x}}{\mathrm{L}}\right),
$$

se llega a las ecuaciones

$$
\frac{\mathrm{dz}}{\mathrm{dt}}+\alpha\left(\frac{\mathrm{n} \pi}{\mathrm{L}}\right)^{2} \mathrm{z}_{\mathrm{n}}=-\frac{\mathrm{v}^{2}}{2 \mathrm{n} \pi \alpha} \exp \left(\frac{\mathrm{v}^{2} \mathrm{t}}{4 \alpha}\right) .
$$

Haciendo uso de las condiciones iniciales $\mathrm{z}_{\mathrm{n}}(0)=0$ se llega a las soluciones

$$
\mathrm{z}_{\mathrm{n}}=\frac{\frac{\mathrm{v}^{2}}{4 \alpha \mathrm{n} \pi}}{\frac{\mathrm{v}^{2}}{4 \alpha}+\alpha\left(\frac{\mathrm{n} \pi}{\mathrm{L}}\right)^{2}}\left(\exp \left(-\alpha \mathrm{t}\left(\frac{\mathrm{n} \pi}{\mathrm{L}}\right)^{2}\right)-\exp \left(\frac{\mathrm{v}^{2}}{4 \alpha} \alpha \mathrm{t}\right)\right) .
$$


Deshaciendo los cambios anteriores la solución del problema inicial se expresa de la forma

$$
\begin{aligned}
\mathrm{T}(\mathrm{x}, \mathrm{t})=\exp \left(\frac{\mathrm{v}}{2 \alpha}\left(\mathrm{x}-\frac{\mathrm{t}}{2}\right)\right) & \left\{\left(1-\mathrm{H}\left(\mathrm{x}-\frac{\mathrm{L}}{2}\right)\right) \exp \left(\frac{\mathrm{v}^{2} \mathrm{t}}{4 \alpha}\right)+\sum_{\mathrm{n}=0}^{\infty} \mathrm{c}_{\mathrm{n}} \operatorname{sen}\left(\frac{\mathrm{n} \pi \mathrm{x}}{\mathrm{L}}\right) \exp \left(-\alpha\left(\frac{\mathrm{n} \pi}{\mathrm{L}}\right)^{2} \mathrm{t}\right)\right. \\
& \left.+\sum_{\mathrm{n}=1}^{\infty} \mathrm{z}_{\mathrm{n}} \operatorname{sen}\left(\frac{\mathrm{n} \pi \mathrm{x}}{\mathrm{L}}\right)\right\},
\end{aligned}
$$

donde los coeficientes $c_{n}$ y $z_{n}$ vienen dados por las expresiones (4.39) y (4.44) respectivamente.

\subsubsection{Solución numérica}

Para obtener la solución numérica al problema se deben analizar los pasos a seguir establecidos en la figura 4.3 en cuanto a la discretización del problema y al método de resolución a utilizar para resolver el sistema de ecuaciones resultante de la discretización.

De forma similar a como se ha hecho para la ecuación de la difusión se utilizan las aproximaciones de las derivadas espaciales y temporales siguientes

$$
\begin{aligned}
& \frac{\partial^{2} \mathrm{~T}(\mathrm{x}, \mathrm{t})}{\partial \mathrm{x}^{2}} \approx \frac{1}{(\Delta \mathrm{x})^{2}}(\mathrm{~T}(\mathrm{x}+\Delta \mathrm{x}, \mathrm{t})-2 \mathrm{~T}(\mathrm{x}, \mathrm{t})+\mathrm{T}(\mathrm{x}-\Delta \mathrm{x}, \mathrm{t})) \\
& \frac{\partial \mathrm{T}(\mathrm{x}, \mathrm{t})}{\partial \mathrm{x}} \approx \frac{1}{2 \Delta \mathrm{x}}(\mathrm{T}(\mathrm{x}+\Delta \mathrm{x}, \mathrm{t})-\mathrm{T}(\mathrm{x}-\Delta \mathrm{x}, \mathrm{t})) \\
& \frac{\partial \mathrm{T}}{\partial \mathrm{t}} \approx \frac{\mathrm{T}(\mathrm{x}, \mathrm{t}+\Delta \mathrm{t})-\mathrm{T}(\mathrm{x}, \mathrm{t})}{\Delta \mathrm{t}}
\end{aligned}
$$

La región de estudio se divide en celdas o nodos de igual modo a como se ha hecho en el caso de la ecuación de la difusión, y también se introduce la notación $\mathrm{T}\left(\mathrm{x}_{\mathrm{i}}, \mathrm{t}_{\mathrm{n}}\right)=\mathrm{T}_{\mathrm{i}}^{\mathrm{n}}$, $\mathrm{T}\left(\mathrm{x}_{\mathrm{i}}+\Delta \mathrm{x}, \mathrm{t}_{\mathrm{n}}\right)=\mathrm{T}_{\mathrm{i}+1}^{\mathrm{n}}, \mathrm{T}\left(\mathrm{x}_{\mathrm{i}}, \mathrm{t}_{\mathrm{n}}+\Delta \mathrm{t}\right)=\mathrm{T}_{\mathrm{i}}^{\mathrm{n}+1}$.

De este modo, utilizando las aproximaciones dadas por las ecuaciones (4.46) se plantea un esquema numérico para la ecuación de la convección difusión de la forma 


$$
\begin{aligned}
\frac{\mathrm{T}_{\mathrm{i}}^{\mathrm{n}+1}-\mathrm{T}_{\mathrm{i}}^{\mathrm{n}}}{\Delta \mathrm{t}}= & \beta\left[\frac{-\mathrm{v}}{2 \Delta \mathrm{x}}\left(\mathrm{T}_{\mathrm{i}+1}^{\mathrm{n}+1}-\mathrm{T}_{\mathrm{i}-1}^{\mathrm{n}+1}\right)+\alpha \frac{1}{(\Delta \mathrm{x})^{2}}\left(\mathrm{~T}_{\mathrm{i}+1}^{\mathrm{n}+1}-2 \mathrm{~T}_{\mathrm{i}}^{\mathrm{n}+1}+\mathrm{T}_{\mathrm{i}-1}^{\mathrm{n}+1}\right)\right] \\
& +(1-\beta)\left[\frac{-\mathrm{v}}{2 \Delta \mathrm{x}}\left(\mathrm{T}_{\mathrm{i}+1}^{\mathrm{n}}-\mathrm{T}_{\mathrm{i}-1}^{\mathrm{n}}\right)+\alpha \frac{1}{(\Delta \mathrm{x})^{2}}\left(\mathrm{~T}_{\mathrm{i}+1}^{\mathrm{n}}-2 \mathrm{~T}_{\mathrm{i}}^{\mathrm{n}}+\mathrm{T}_{\mathrm{i}-1}^{\mathrm{n}}\right)\right]
\end{aligned}
$$

Escribiendo la ecuación (4.47) para los puntos $\mathrm{x}_{1}, \ldots, \mathrm{x}_{\mathrm{N}}$, en los que se divide la región de integración, se obtiene un sistema tridiagonal de ecuaciones de la forma

$$
\left[\begin{array}{ccccccc}
\gamma_{2} & \gamma_{3} & 0 & 0 & 0 & \cdots & 0 \\
\gamma_{1} & \gamma_{2} & \gamma_{3} & 0 & 0 & \cdots & 0 \\
0 & \gamma_{1} & \gamma_{2} & \gamma_{3} & 0 & \cdots & 0 \\
\vdots & & & \ddots & & & \vdots \\
0 & \cdots & 0 & \gamma_{1} & \gamma_{2} & \gamma_{3} & 0 \\
0 & \cdots & 0 & 0 & \gamma_{1} & \gamma_{2} & \gamma_{3} \\
0 & \cdots & 0 & 0 & 0 & \gamma_{1} & \gamma_{2}
\end{array}\right]\left[\begin{array}{c}
\mathrm{T}_{1}^{\mathrm{n}+1} \\
\mathrm{~T}_{2}^{\mathrm{n}+1} \\
\mathrm{~T}_{3}^{\mathrm{n}+1} \\
\vdots \\
\mathrm{T}_{\mathrm{n}-2}^{\mathrm{n}-1} \\
\mathrm{~T}_{\mathrm{n}-1}^{\mathrm{n}-1} \\
\mathrm{~T}_{\mathrm{N}}^{\mathrm{n}+1}
\end{array}\right]=\left[\begin{array}{c}
\mathrm{d}_{1} \\
\mathrm{~d}_{2} \\
\mathrm{~d}_{3} \\
\vdots \\
\mathrm{d}_{\mathrm{N}-2} \\
\mathrm{~d}_{\mathrm{N}-1} \\
\mathrm{~d}_{\mathrm{N}}
\end{array}\right]
$$

siendo

$$
\begin{aligned}
& \gamma_{1}=-\beta\left[\frac{\mathrm{v}}{2 \Delta \mathrm{x}}+\frac{\alpha}{(\Delta \mathrm{x})^{2}}\right] \\
& \gamma_{2}=\frac{1}{\Delta \mathrm{t}}+\frac{\alpha \beta}{(\Delta \mathrm{x})^{2}} \\
& \gamma_{3}=\beta\left[\frac{\mathrm{v}}{2 \Delta \mathrm{x}}-\frac{\alpha}{(\Delta \mathrm{x})^{2}}\right] \\
& \mathrm{d}_{1}=\frac{\mathrm{v}}{2 \Delta \mathrm{x}}+\frac{\alpha}{(\Delta \mathrm{x})^{2}}+\mathrm{T}_{1}^{\mathrm{n}}\left[\frac{1}{\Delta \mathrm{t}}-\frac{2(1-\beta) \alpha}{(\Delta \mathrm{x})^{2}}\right]+\mathrm{T}_{2}^{\mathrm{n}}(1-\beta)\left[-\frac{\mathrm{v}}{2 \Delta \mathrm{x}}+\frac{\alpha}{(\Delta \mathrm{x})^{2}}\right]
\end{aligned}
$$

para $2 \leqslant \mathrm{i} \leqslant \mathrm{N}-1$

$$
\mathrm{d}_{\mathrm{i}}=\mathrm{T}_{\mathrm{i}-1}^{\mathrm{n}}(1-\beta)\left[\frac{\mathrm{v}}{2 \Delta \mathrm{x}}+\frac{\alpha}{(\Delta \mathrm{x})^{2}}\right]+\mathrm{T}_{\mathrm{i}}^{\mathrm{n}}\left[\frac{1}{\Delta \mathrm{t}}-\frac{2(1-\beta) \alpha}{(\Delta \mathrm{x})^{2}}\right]+\mathrm{T}_{\mathrm{i}+1}^{\mathrm{n}}(1-\beta)\left[-\frac{\mathrm{v}}{2 \Delta \mathrm{x}}+\frac{\alpha}{(\Delta \mathrm{x})^{2}}\right]
$$




$$
\mathrm{d}_{\mathrm{N}}=\mathrm{T}_{\mathrm{N}-1}^{\mathrm{n}}\left[\frac{\mathrm{v}}{2 \Delta \mathrm{x}}+\frac{\alpha}{(\Delta \mathrm{x})^{2}}\right]+\mathrm{T}_{\mathrm{N}}^{\mathrm{n}}\left[\frac{1}{\Delta \mathrm{t}}-\frac{2(1-\beta) \alpha}{(\Delta \mathrm{x})^{2}}\right]
$$

De la misma forma que en el caso de la ecuación de la difusión, el esquema de resolución explícito se ha desestimado por los problemas de inestabilidad, y se ha optado también por un método de resolución en diferencias finitas implícito, $\beta=1$, y el de Crank-Nicolson, $\beta=0.5$ que son esquemas estables para cualquier valor de la velocidad y de la difusividad (Fletcher1991).

\subsubsection{Optimización de parámetros y estudios de sensibilidad}

Tal como se ha hecho en el problema de la estimación de parámetros para la ecuación de la difusión, analizaremos las capacidades del método de búsqueda multidireccional y del algoritmo genético, para la búsqueda de los parámetros de velocidad de convección, v, y coeficiente de difusividad, $\alpha$, la ecuación de la convección-difusión.

Tomaremos como referencia los valores proporcionados por la solución analítica (4.45), considerando los 100 primeros términos de la serie infinita. Para esta solución analítica se han fijado los valores $v=0.3 \mathrm{~m} / \mathrm{s}$ y $\alpha=0.1 \mathrm{~m}^{2} / \mathrm{s}$, y se ha tomado como tiempo final de la simulación $\mathrm{TF}=0.1 \mathrm{~s}$. Esta elección no introduce ningún tipo de error puesto que los problemas de oscilaciones de la serie desaparecen al considerar la suma con un número suficiente de términos.

En primer lugar se han realizado los análisis de sensibilidad utilizando el algoritmo MDS, el cual se ha implementado con las características que figuran en la tabla 4.9.

\begin{tabular}{ll}
\hline Características MDS & Valores \\
\hline Símplice inicial & $0.5,0.0$ \\
& $0.0,0.0$ \\
& $0.4,0.4$ \\
Paso contracción & 0.5 \\
Paso expansión & 2.0 \\
Iteraciones máximas & 200 \\
Evaluaciones máximas & 1600 \\
Tolerancia & $1.010^{-5}$ \\
\hline
\end{tabular}

Tabla 4.9: Características del MDS para 2 parámetros. 
Debido al significado físico de los parámetros a optimizar se ha restringido el rango de variación de los mismos de manera que no se permite que adopten valores negativos. En el caso de la difusividad no tiene sentido considerar valores negativos, y en el caso de la velocidad imponiendo la restricción de que sea siempre positiva aseguramos que el fluido se mueve siempre en la misma dirección y evitamos que se produzca flujo contracorriente. Además, al ser las variables del mismo orden de magnitud no es necesaria la escalación del problema.

Al igual que para la ecuación de la difusión, se han realizado diversos análisis de sensibilidad con el fin de determinar la influencia de los parámetros del esquema numérico, es decir, el número de nodos espaciales, $\mathrm{N}$, y el paso de tiempo, DT, también del número de detectores, controlado por KDTEC, y del periodo de muestreo, que viene dado por DDT.

Así, utilizando como método de optimización el MDS se han realizado diversos análisis de sensibilidad como el que se muestra en la tabla 4.10 en el que se ha cambiado el número de nodos manteniendo constantes los otros parámetros con los valores KDTEC=1, $\mathrm{DT}=0.0001, \mathrm{DDT}=0.1, \mathrm{TINI}=0.0$.

\begin{tabular}{|c|c|c|c|c|c|}
\hline & & \multicolumn{2}{|c|}{ Crank-Nicolson: $\beta=0.5$} & \multicolumn{2}{|c|}{ Implícito: $\beta=1.0$} \\
\hline & & Norma Inf & Norma 1 & Norma Inf & Norma 1 \\
\hline \multicolumn{6}{|c|}{$\mathrm{DT}=0.0001 \mathrm{DDT}=0.1 \mathrm{KDTEC}=1$} \\
\hline \multirow[t]{5}{*}{$\mathrm{N}=31$} & valor alfa & 0.10105 & 0.09487 & 0.10063 & 0.09478 \\
\hline & valor $\mathrm{v}$ & 0.51031 & 0.51367 & 0.50976 & 0.51786 \\
\hline & error & 0.00789 & 0.00890 & 0.00785 & 0.00888 \\
\hline & evaluaciones & 150 & 136 & 115 & 143 \\
\hline & tiempo (seg.) & 4.02 & 2.74 & 2.16 & 2.78 \\
\hline \multirow[t]{5}{*}{$\mathrm{N}=63$} & valor alfa & 0.09955 & 0.09426 & 0.09948 & 0.09463 \\
\hline & valor v & 0.42775 & 0.51367 & 0.42773 & 0.44043 \\
\hline & error & 0.0083 & 0.00884 & 0.00828 & 0.00892 \\
\hline & evaluaciones & 185 & 143 & 101 & 122 \\
\hline & tiempo (seg.) & 7.44 & 5.68 & 4.96 & 4.71 \\
\hline \multirow[t]{5}{*}{$\mathrm{N}=127$} & valor alfa & 0.09989 & 0.09454 & 0.09935 & 0.0943 \\
\hline & valor v & 0.42775 & 0.39548 & 0.38964 & 0.40063 \\
\hline & error & 0.00836 & 0.00890 & 0.00833 & 0.00889 \\
\hline & evaluaciones & 136 & 178 & 171 & 136 \\
\hline & tiempo (seg.) & 11.06 & 14.31 & 14.50 & 10.59 \\
\hline \multirow[t]{5}{*}{$\mathrm{N}=255$} & valor alfa & 0.09936 & 0.09423 & 0.09974 & 0.09447 \\
\hline & valor v & 0.36523 & 0.37988 & 0.37011 & 0.37796 \\
\hline & error & 0.00845 & 0.00885 & 0.00831 & 0.00886 \\
\hline & evaluaciones & 150 & 192 & 122 & 234 \\
\hline & tiempo (seg.) & 24.47 & 31.04 & 19.36 & 36.83 \\
\hline
\end{tabular}

Tabla 4.10: Análisis de la dependencia del número de nodos. 
En la tabla 4.10 se observa que el algoritmo MDS encuentra valores de la difusividad cercanos al de referencia aunque los intervalos de discretización espacial sean grandes. No ocurre así con el valor de la velocidad que, en el caso de considerar intervalos espaciales grandes, $\mathrm{N}$ pequeño, el valor óptimo encontrado para minimizar el error cometido en el cálculo de la temperatura es bastante mayor que el de referencia, tomado como 0.3, para cualquiera de las normas utilizadas, aproximándose a este valor a medida que se aumentan el número de nodos, $\mathrm{N}$.

Como ya se mencionó en el caso anterior, este resultado es de gran importancia en la aplicación a códigos termohidráulicos puesto que al disponer de una determinada nodalización los resultados numéricos que más se ajustan a los datos medidos no son necesariamente los calculados con los parámetros de referencia.

La comprobación de que con los valores proporcionados por el MDS tras al optimización, la evolución de la temperatura se ajusta más a la experimental que el cálculo que resulta con los valores de referencia, se muestra en la figura 4.9, en la que se presenta el perfil de temperatura para una nodalización de $\mathrm{N}=15$ considerando los valores de la referencia y con los que proporciona el MDS.

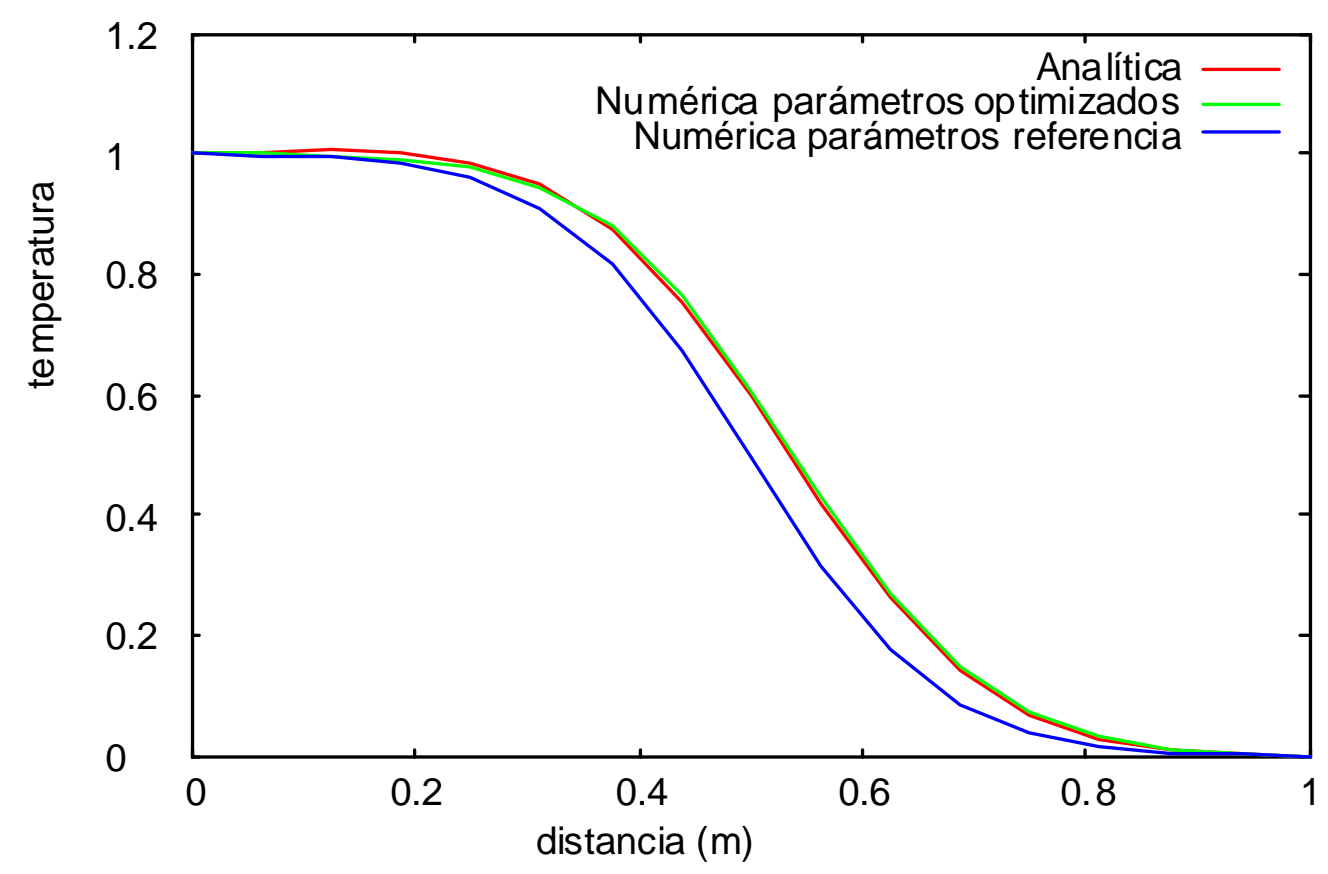

Figura 4.9: Perfiles de temperatura calculados con respecto al de referencia. 
La figura 4.9 muestra que el perfil de temperatura proporcionado por cálculos numéricos realizados con los valores de referencia de la difusividad, $\alpha=0.1$, y de la velocidad, $\mathrm{v}=0.3$, presentan un retardo con respecto a la curva de la temperatura de la solución analítica, que en este caso actúa como datos experimentales. Sin embargo, los valores óptimos que encuentra el MDS para la difusividad y para la velocidad, aunque son distintos a los valores de referencia, calculan un perfil de temperatura mucho más ajustado a la solución analítica.

Con el fin de determinar la influencia en los cálculos del paso de tiempo de la numérica se han realizado las optimizaciones para dos valores extremos del número de nodos con un tiempo DT $=0.001,10$ veces mayor que el anterior, manteniendo todos los demás valores como en el caso anterior. Los resultados obtenidos se presentan en la tabla 4.11.

\begin{tabular}{llllll}
\hline \multicolumn{5}{c}{ Crank-Nicolson: $\beta=0.5$} & \multicolumn{2}{c}{ Implícito: $\beta=1.0$} \\
\hline & Norma Inf & Norma 1 & Norma Inf & Norma 1 \\
\hline $\mathrm{DT}=0.001$ DDT=0.1 KDTEC=1 & & & \\
\hline $\mathrm{N}=31$ & valor alfa & 0.10063 & 0.09478 & 0.10161 & 0.09454 \\
& valor v & 0.50976 & 0.51825 & 0.51074 & 0.51611 \\
& error & 0.00792 & 0.00891 & 0.00752 & 0.00896 \\
& evaluaciones & 108 & 143 & 122 & 108 \\
& tiempo (seg.) & 0.64 & 0.86 & 0.72 & 0.64 \\
\hline $\mathrm{N}=225$ & valor alfa & 0.09937 & 0.09429 & 0.10035 & 0.09437 \\
& valor v & 0.36914 & 0.38156 & 0.36987 & 0.3789 \\
& error & 0.00838 & 0.00887 & 0.00809 & 0.00896 \\
& evaluaciones & 136 & 192 & 164 & 248 \\
& tiempo (seg.) & 6.91 & 9.66 & 8.26 & 12.39 \\
\hline
\end{tabular}

Tabla 4.11: Análisis de la dependencia del paso temporal de integración (1).

Al comparar los resultados expuestos en la tabla 4.11 con los de la tabla 4.10 se observa que el valor del error cometido es en ambos casos del mismo orden de magnitud, así como los valores de difusividad y velocidad que minimizan este error. Sin embargo al aumentar el paso temporal el tiempo invertido en las optimizaciones es bastante menor en este último caso, por lo que se deduce que el tiempo estimado en un principio es excesivamente pequeño y que no proporciona ninguna mejora en el resultado sino que únicamente disminuye la rapidez del cálculo.

Ante este resultado se realizaron los mismos casos que aparecen en la tabla 4.11 para tiempos de integración DT=0.01 y DT=0.1, obteniéndose los resultados que se muestran en la tabla 4.12 . 


\begin{tabular}{|c|c|c|c|c|c|}
\hline & & \multicolumn{2}{|c|}{ Crank-Nicolson: $\beta=0.5$} & \multicolumn{2}{|c|}{ Implícito: $\beta=1.0$} \\
\hline & & Norma Inf & Norma 1 & Norma Inf & Norma 1 \\
\hline \multicolumn{6}{|l|}{$\mathrm{DT}=0.01$} \\
\hline \multirow[t]{5}{*}{$\mathrm{N}=31$} & valor alfa & 0.10062 & 0.09478 & 0.10261 & 0.09871 \\
\hline & valor v & 0.5097 & 0.51831 & 0.52539 & 0.53009 \\
\hline & Error & 0.00805 & 0.00889 & 0.00705 & 0.00976 \\
\hline & evaluaciones & 115 & 150 & 150 & 164 \\
\hline & tiempo (seg.) & 0.53 & 0.71 & 0.68 & 0.77 \\
\hline \multirow[t]{5}{*}{$\mathrm{N}=255$} & valor alfa & 0.09398 & 0.09317 & 0.10281 & 0.09844 \\
\hline & valor $\mathrm{v}$ & 0.371098 & 0.21356 & 0.3833 & 0.39233 \\
\hline & Error & 0.01496 & 0.16884 & 0.00966 & 0.01009 \\
\hline & evaluaciones & 94 & 178 & 115 & 164 \\
\hline & tiempo (seg.) & 4.72 & 6.77 & 4.55 & 6.42 \\
\hline \multicolumn{6}{|l|}{$\mathrm{DT}=0.1$} \\
\hline \multirow{5}{*}{$\mathrm{NX}=31$} & valor alfa & 0.093041 & 0.25125 & 0.12604 & 0.12644 \\
\hline & valor $\mathrm{v}$ & 0.62231 & 0.62890 & 0.52246 & 0.66186 \\
\hline & Error & 0.07335 & 0.40224 & 0.03889 & 0.04138 \\
\hline & evaluaciones & 150 & 94 & 136 & 220 \\
\hline & tiempo (seg.) & 0.66 & 0.43 & 0.6 & 1.00 \\
\hline \multirow[t]{5}{*}{$\mathrm{Nx}=255$} & valor alfa & 0.10057 & 0.250911 & 0.12780 & 0.14698 \\
\hline & valor v & 0.4843 & 0.45568 & 0.42773 & 0.46533 \\
\hline & error & 0.07684 & 0.48460 & 0.03421 & 0.03811 \\
\hline & evaluaciones & 171 & 108 & 178 & 136 \\
\hline & tiempo (seg.) & 6.10 & 4.09 & 6.57 & 5.17 \\
\hline
\end{tabular}

Tabla 4.12: Análisis de la dependencia del paso de temporal de integración (2).

En esta tabla se observa que para valores de DT $=0.01$ los resultados siguen siendo parecidos a los obtenidos con pasos menores, si bien es cierto que se observa un ligero aumento del error, los valores de las variables para el error mínimo son muy similares a los de la tabla 4.11. Finalmente, en el caso en el que DT $=0.1$ se observa un mayor aumento del error calculado y con una diferencia en los valores de las variables que hacen mínimo el error. Además, este caso no presenta una mejora en cuanto al tiempo de CPU invertido con respecto a los anteriores.

Por tanto, la elección del paso de integración es importante puesto que tiempos excesivamente pequeños únicamente aumentan el tiempo de simulación sin mejorar los resultados y con tiempos excesivamente grandes aumenta el error cometido sin proporcionar una reducción del tiempo de cálculo. Esto es importante a tener en cuenta en la definición del paso de avance de los códigos termohidráulicos, puesto que en algunos transitorios pueden llevar a tiempos de cálculo prohibitivos. 
Del mismo modo que en el caso anterior, en la optimización de los parámetros de la ecuación de la convección difusión se ha utilizado un algoritmo genético de estado estacionario (AGEE), aunque como el problema a resolver es diferente los parámetros que utiliza el algoritmo deben ajustarse a las nuevas condiciones del problema. En este caso los parámetros del algoritmo utilizados en los cálculos se muestran en la tabla 4.13.

\begin{tabular}{ll}
\hline Parámetro del AGEE & \\
\hline Codificación & Real \\
Tamaño población & 50 \\
Probabilidad de cruce & 0.6 \\
Probabilidad de mutación & 0.5 \\
Número de máximo de generaciones & 500 \\
Porcentaje de convergencia & 0.95 \\
Porcentaje de reemplazamiento & 0.25 \\
Método de selección & GATournamentSelector \\
Método de cruce & OnePointCrossover \\
Método de mutación & FlipMutator \\
Método de escalado & GAPowerLawScaling \\
Método de terminación & UponConvergenceUPV \\
\hline
\end{tabular}

Tabla 4.13: Características del AGEE para 2 parámetros.

También en este caso la población inicial la componen 50 individuos de los cuales se han inicializado tres de ellos a los valores que definen el símplice inicial del algoritmo MDS, presentados en la tabla 4.9, mientras que la población restante la elige el AGEE de manera aleatoria. Esta inicialización pretende que el algoritmo al iniciar la búsqueda tenga la misma información de la que parte el MDS. Puesto que las dos variables de las que depende el problema toman valores reales, se ha elegido la codificación real implementada en el GALib para representar los individuos de la población, los cuales están formado por dos genes que representa la difusividad y la velocidad.

En cuanto al criterio de terminación, se han elegido 500 generaciones como el máximo a realizar aunque no se haya alcanzado la convergencia, un porcentaje de convergencia de la población del 0.95 y un valor para la convergencia genética de 0.99 .

Puesto que en los análisis de sensibilidad realizados con el método MDS se observa que la solución al problema de optimización presenta una dependencia mayor con respecto a la discretización espacial de la numérica, se ha reproducido este análisis utilizando el AGEE con las características descritas anteriormente. 
En este análisis se varia el número de nodos considerando los valores de los demás parámetros fijos y con valores: $\mathrm{KDTEC}=1, \mathrm{TF}=0.1, \mathrm{NTER}=100, \mathrm{DDT}=0.1, \mathrm{DT}=0.0001$, TINI=0.0. Los resultados de las optimizaciones llevadas a cabo con el AGEE se muestran en la tabla 4.14.

\begin{tabular}{llllll}
\hline & & \multicolumn{2}{l}{ Crank-Nicolson: $\beta=0.5$} & \multicolumn{2}{c}{ Implícito: $\beta=1.0$} \\
\hline \multirow{3}{*}{ =31 } & Norma Inf & Norma 1 & Norma Inf & Norma 1 \\
& valor alfa & 0.103 & 0.097 & 0.1 & 0.096 \\
\hline & valor v & 0.499 & 0.495 & 0.499 & 0.499 \\
& error & 0.00888 & 0.00948 & 0.00874 & 0.00927 \\
& generaciones & 500 & 168 & 500 & 480 \\
& tiempo (seg.) & 294.43 & 100.52 & 290.71 & 279.65 \\
\hline $\mathrm{N}=63$ & valor alfa & 0.1 & 0.096 & 0.1 & 0.096 \\
& valor v & 0.431 & 0.446 & 0.43 & 0.442 \\
& error & 0.00836 & 0.00905 & 0.00829 & 0.00917 \\
& generaciones & 358 & 175 & 229 & 172 \\
& tiempo (seg.) & 431.43 & 214.51 & 274.47 & 207.55 \\
\hline $\mathrm{N}=127$ & valor alfa & 0.099 & 0.096 & 0.1 & 0.095 \\
& valor v & 0.39 & 0.446 & 0.43 & 0.442 \\
& error & 0.00841 & 0.00908 & 0.00841 & 0.00898 \\
& generaciones & 137 & 265 & 213 & 196 \\
& tiempo (seg.) & 339.96 & 648.34 & 516.68 & 476.66 \\
\hline $\mathrm{N}=255$ & valor alfa & 0.098 & 0.095 & 0.099 & 0.096 \\
& valor v & 0.371 & 0.384 & 0.37 & 0.382 \\
& error & 0.00856 & 0.00902 & 0.00834 & 0.00910 \\
& generaciones & 168 & 224 & 289 & 205 \\
& tiempo (seg.) & 835.43 & 1107.69 & 1407.07 & 1004.14 \\
\hline
\end{tabular}

Tabla 4.14: Análisis de la dependencia del numero de nodos.

Los valores a los que llega el AGEE son similares a los proporcionados por el MDS, observándose la misma tendencia en cuanto al valor a que tiende la velocidad para minimizar el error del cálculo. Sin embargo, en este caso el tiempo de cálculo necesario para alcanzar los valores más adecuados de los parámetros de optimización es mucho mayor que el necesario con el MDS, aproximadamente dos órdenes de magnitud mayor. Por esta razón, se puede concluir que para este tipo de problemas el AGEE es altamente ineficiente frente al método MDS, puesto que aunque los resultados obtenidos para las variables son similares en ambos casos el tiempo invertido hace desaconsejable su uso. 
La forma de la función objetivo explica esta diferencia en cuanto al tiempo de cálculo necesario. En la figura 4.10 está representado el error cuantificado mediante la Norma 1 en función de la difusividad, alfa, y la velocidad, v. En esta figura se observa que la función error presenta una dependencia clara de la difusividad, mientras que con respecto a la velocidad esta variación no es tan pronunciada. Además, la zona en la que se encuentra el mínimo es muy plana, lo que indica que existen muchas combinaciones de parámetros con un error muy similar, lo que explica que el AGEE encuentre muchos puntos con valores similares y por tanto la búsqueda no se dirija rápidamente hacia el mínimo sino que intente explorar las zonas próximas a muchos puntos y se favorezca la convergencia prematura del algoritmo. En cambio el MDS, al realizar la búsqueda siguiendo unas determinadas direcciones avanza más rápidamente hacia el óptimo. Las otras normas utilizadas tienen la misma forma que ésta y por tanto el comportamiento de los métodos de búsqueda es el mismo.

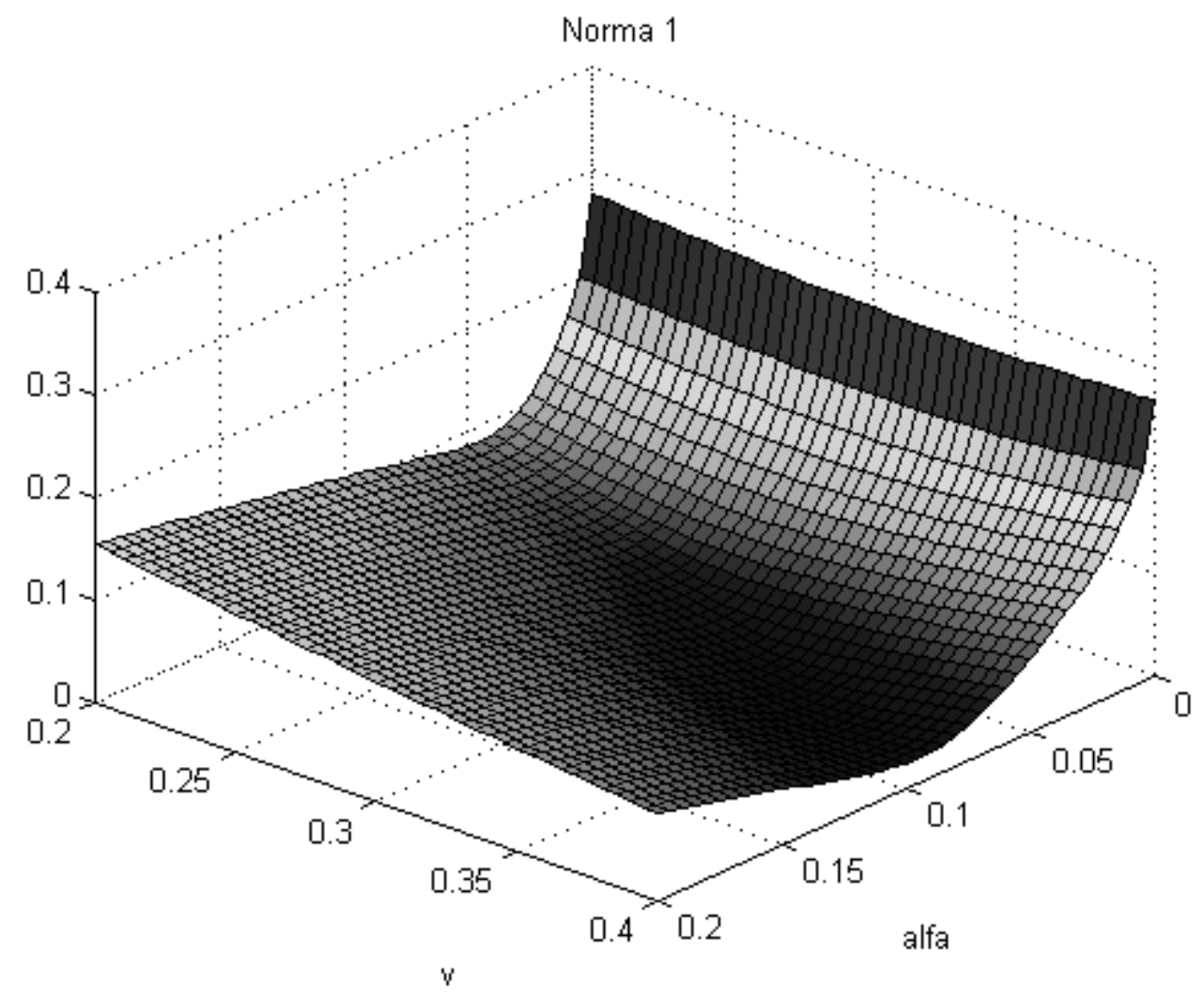

Figura 4.10: Función error utilizando la Norma 1.

Esta situación es del todo extrapolable a los problemas de simulación habituales mediante códigos de estimación óptima por lo que parece haber una indicación clara de que con el algoritmo MDS se alcanzarán buenos resultados con una clara ventaja en términos del tiempo de cálculo. 
Capítulo 4 


\section{Capítulo 5}

\section{Aplicación a simulaciones mediante RELAP5/Mod3.2}

\subsection{Introducción}

La aplicación de la metodología de optimización es de gran interés en la eliminación de algunos de los efectos que introducen incertidumbres en el proceso de simulación del comportamiento de una central nuclear ante un accidente determinado, tal como se manifiesta en las metodologías desarrolladas para el cálculo de estas incertidumbres (Boyack1990; Catton1990; Wilson 1990; Wulf 1990; Zuber 1990; D'Auria1995). Algunos de los errores a los que pueden conducir los cálculos realizados mediante un código termohidráulico pueden ser debidas a factores que aplicando una búsqueda sistemática pueden eliminarse fácilmente. En concreto se podrían introducir errores debido al hecho que el modelo de planta que se utiliza en la simulación no es el más idóneo.

En cualquier simulación realizada con un código termohidráulico aparecen incertidumbres que pueden deberse tanto a que los modelos matemáticos que utilizan no reproducen los fenómenos físicos reales, como a que los parámetros del modelo no son los más adecuados. Con respecto a este último problema que puede surgir al analizar los resultados de una simulación, la única solución consiste en realizar diversos análisis de sensibilidad, tal como se plantea en las metodologías de cálculo de incertidumbres (Boyack1990; Catton1990; Wilson 1990; Wulf 1990; Zuber 1990; D'Auria1995), con el fin de detectar la combinación de los valores de los parámetros que proporcionan una solución más parecida a la experimental. 
Actualmente este proceso de búsqueda se realiza de forma manual, con lo que se ve afectado por la introducción de numerosos efectos de usuario que, utilizando un proceso automático como el que aquí se presenta, podrían minimizarse o incluso eliminarse.

La metodología que se plantea empieza con la identificación de aquellos parámetros del modelo cuya variación puede afectar de manera significativa al resultado del cálculo proporcionado por el código termohidráulico utilizado. En este capítulo se utiliza el código termohidráulico RELAP5/Mod3.2 (INEL1995a; INEL1995b; INEL1995c; INEL1995d; INEL1996).

Una vez que se han identificado los parámetros que pueden afectar a la solución obtenida, y los rangos de variación en los que puede tomar valores para que el parámetro tenga significado físico, empieza la búsqueda propiamente dicha. En los casos de aplicación que se han realizado, el método elegido para realizar la optimización de parámetros ha sido el método de búsqueda multidireccional (MDS) puesto que al utilizar el código RELAP5 la evaluación de la función objetivo requiere de un tiempo de computación considerable, a pesar de utilizar un modelo simple de la instalación y simular pocos segundos de transitorio, y el MDS presenta una clara ventaja sobre el AGEE para resolver este tipo de problemas.

La función objetivo que evalúa el MDS es el error entre una cierta variable de referencia, por ejemplo las medidas experimentales, y el valor de la misma variable calculada con el RELAP5/Mod3.2, para cada combinación de valores de los parámetros a optimizar, los cuales se especifican en el fichero de entrada diseñado para una determinada simulación. En concreto, cada vez que el MDS realiza un cálculo de la función objetivo con el fin de obtener el error cometido en la simulación se siguen los siguientes pasos:

1.- Lectura del fichero de entrada de datos al código RELAP5/Mod3.2 y cambio del valor del parámetro, o parámetros, en la tarjeta y posición requerida.

2.- Ejecución del código y obtención de los valores calculados para la variable que debe compararse con la referencia.

3.- Cálculo del error cometido en la simulación, que es el valor que adopta el método MDS para tomar decisiones respecto a la bondad de la combinación de parámetros considerada. 
El procedimiento anterior describe de forma general el funcionamiento de la optimización de parámetros haciendo uso de un código termohidráulico. En este punto, para acabar de definir la función objetivo a optimizar es necesaria la determinación del transitorio que debe realizar el RELAP5. En nuestro caso, con la finalidad de que tanto los transitorios simulados como los resultados alcanzados en el proceso de optimización tengan significado desde el punto de vista termohidráulico, se han buscado ejemplos incluidos dentro de las matrices de validación del código RELAP5.

\subsection{Matrices de validación y casos de aplicación}

En las matrices que se utilizan en el proceso de validación del código se establecen los fenómenos físicos que debe reproducir el código, los tipos de experimentos realizados y las instalaciones en los que han tenido lugar, con lo que constituyen una guía a seguir para la revisión y comprobación de los modelos que han sido implementados. Con los resultados obtenidos se consigue determinar las capacidades y limitaciones del código para simular adecuadamente los transitorios que pueden producirse en las centrales nucleares.

Dentro del proceso de validación es fundamental la comparación de los resultados que proporciona el código con datos experimentales. Existen dos tipos de experimentos que se llevan a cabo durante el proceso de validación de un código:

- Experimentos de efectos separados. El objetivo de estos experimentos es analizar, siempre que sea posible, por separado cada uno de los modelos implementados en el código. Estos experimentos sirven para analizar los efectos de un determinado fenómeno físico o de una fase concreta del transitorio.

- Experimentos de instalaciones integrales. Se realizan con el objetivo de observar la interrelación entre los diferentes fenómenos físicos que tienen lugar al producirse un determinado transitorio en una planta a escala reducida.

El proceso de validación de un código debe cubrir un rango de condiciones de funcionamiento apropiado, como guía para valorar si el código es capaz de simular las condiciones para las que ha sido diseñado. La OECD/CSNI ha establecido las denominadas matrices de valoración, en las que representan el experimento realizado, el fenómeno físico que debe reproducir y la instalación en la que se realiza el experimento (INEL1996). Estas matrices pueden completarse con otras instalaciones experimentales en las que se han llevado a cabo experimentos realizados dentro de los programas 
internacionales de valoración y mantenimiento de los códigos como el CAMP o el ICAP.

En particular, para el caso del código termohidráulico RELAP5/Mod3, los estudios de validación realizados aplicables a reactores occidentales de agua ligera a presión (PWR) se muestran en las tablas 5.1 y 5.2. En estas tablas se indican los fenómenos físicos que tienen lugar en una central nuclear al producirse una pérdida de refrigerante debido a una rotura grande (LBLOCA) o pequeña (SBLOCA) y la capacidad del código para reproducir estos fenómenos para cada una de las instalaciones experimentales. En total en las tablas se recogen los resultados de diez experimentos de efectos separados, seis experimentos en instalaciones integrales, un transitorio de planta y un experimento tipo en el que se comparan los resultados con los de una base de datos.

\begin{tabular}{|c|c|c|c|c|c|c|c|}
\hline \multirow{2}{*}{\multicolumn{2}{|c|}{$\begin{array}{l}\text { MATRIZ I } \\
\text { Matriz de referencia cruzada para LBLOCAs en PWRs } \\
\text { Fenómeno/Experimento } \\
\text { - Simulado } \\
\text { o Parcialmente simulado } \\
\text { - No simulado } \\
\text { Fenómeno/Instalación } \\
\text { - Adecuada para la valoración del código } \\
\text { o Parcialmente adecuada } \\
\text { - No simulado }\end{array}$}} & \multicolumn{3}{|c|}{$\begin{array}{l}\text { tipo de } \\
\text { experimento }\end{array}$} & \multicolumn{3}{|c|}{$\begin{array}{l}\text { Instalación de } \\
\text { efectos separados }\end{array}$} \\
\hline & & $\begin{array}{l}\frac{8}{0} \\
. \frac{\pi}{\pi} \\
\stackrel{\pi}{>}\end{array}$ & 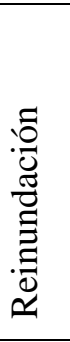 & 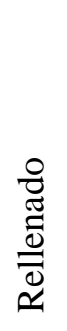 & $\underline{\underline{a}}$ & $\frac{\vec{T}}{\frac{5}{5}}$ & 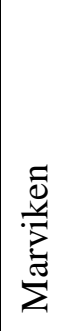 \\
\hline \multirow{13}{*}{ 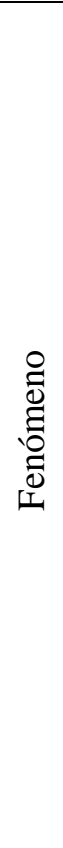 } & caudal de fuga & - & - & - & - & - & $\bullet$ \\
\hline & separación de fases & - & - & - & - & - & - \\
\hline & condensación y mezcla durante la inyección & - & - & - & - & - & - \\
\hline & $\begin{array}{l}\text { amplio descubrimiento del núcleo + distribución de } \\
\text { flujo }\end{array}$ & - & - & - & - & - & - \\
\hline & $\begin{array}{l}\text { bypass y penetración del sistema de refrigeración de } \\
\text { emergencia del núcleo }\end{array}$ & - & • & • & - & - & - \\
\hline & Limitación del flujo contracorriente & - & - & - & - & - & - \\
\hline & Formación de bolsa de vapor & - & - & - & - & - & - \\
\hline & Estancamiento de agua en plenum superior de la vasija & - & - & - & - & - & - \\
\hline & Transferencia de Calor en el núcleo & - & $\bullet$ & $\bullet$ & O & $\bullet$ & - \\
\hline & Propagación del frente de remojado & - & - & O & - & O & - \\
\hline & Suspensión de gotas (núcleo, plenum superior) & - & - & - & - & - & - \\
\hline & Caída de gotas (núcleo, plenum superior) & - & - & - & - & - & - \\
\hline & Comportamiento de las bombas para 1 y 2 fases & - & - & - & - & - & - \\
\hline
\end{tabular}

Tabla 5.1: Matriz de valoración del RELAP5/Mod3.0 para LBLOCAs en PWR. 


\begin{tabular}{|c|c|c|c|c|c|c|c|c|c|c|c|c|c|c|c|}
\hline \multirow{3}{*}{\multicolumn{2}{|c|}{$\begin{array}{l}\text { MATRIZ I } \\
\text { Matriz de referencia cruzada para LBLOCAs en } \\
\text { PWRs } \\
\text { Fenómeno/Experimento } \\
\text { - Simulado } \\
\text { o Parcialmente simulado } \\
\text { - No simulado } \\
\text { Fenómeno/Instalación } \\
\text { - Adecuada para la valoración del código } \\
\text { o Parcialmente adecuada } \\
\text { - No simulado } \\
\text { Instalación/ Exp. tipo } \\
\text { - Simulado } \\
\text { o Parcialmente simulado } \\
\text { - No simulado }\end{array}$}} & \multicolumn{3}{|c|}{ experimento Tipo } & \multicolumn{11}{|c|}{ Instalación } \\
\hline & & \multirow[b]{2}{*}{ 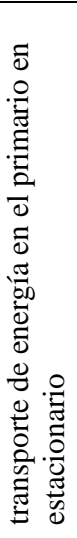 } & \multirow[b]{2}{*}{ 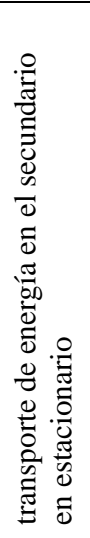 } & \multirow[b]{2}{*}{ 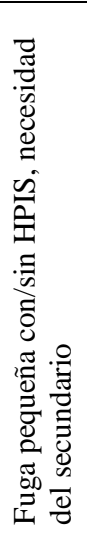 } & \multicolumn{3}{|c|}{ Sistemas } & \multicolumn{7}{|c|}{ Efectos separados } & \multirow[t]{2}{*}{ Planta } \\
\hline & & & & & $\underset{n}{2}$ & 空 & 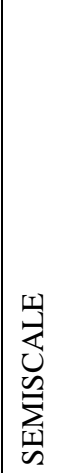 & 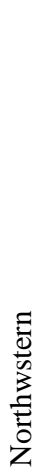 & $\frac{\vec{\alpha}}{\square}$ & 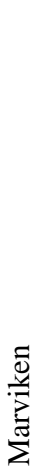 & $\begin{array}{l}U \\
\frac{1}{\infty} \\
3\end{array}$ & 志 & 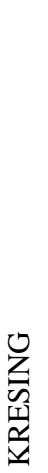 & $\underline{\underline{\alpha}}$ & \\
\hline \multirow{23}{*}{ 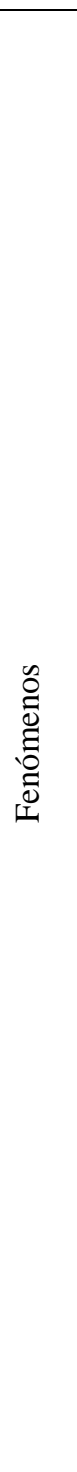 } & Circulación natural primario, 1 fase & $\bullet$ & $\bullet$ & $\bullet$ & $\bullet$ & $\bullet$ & $\bullet$ & - & - & - & O & - & - & - & $\bullet$ \\
\hline & Circulación natural primario, 2 fases & $\bullet$ & $\bullet$ & $\bullet$ & $\bullet$ & $\bullet$ & $\bullet$ & - & - & - & $\mathrm{O}$ & - & - & - & - \\
\hline & Condensación de reflujo y CCFL & $\bullet$ & $\bullet$ & $\bullet$ & $\bullet$ & $\bullet$ & O & - & $\bullet$ & - & - & $\bullet$ & - & - & - \\
\hline & Comportamiento asimétrico del lazo & - & - & $\bullet$ & - & - & O & - & - & - & - & - & - & - & $\bullet$ \\
\hline & Caudal de fuga & - & - & $\bullet$ & - & - & $\bullet$ & - & - & $\bullet$ & - & - & $\bullet$ & - & - \\
\hline & $\begin{array}{l}\text { Separación de fases con/sin formación } \\
\text { de nivel de mezcla }\end{array}$ & $\bullet$ & - & $\bullet$ & • & • & O & - & - & - & - & O & - & - & - \\
\hline & $\begin{array}{l}\text { Nivel de mezcla y suspensión en comp. } \\
\text { verticales del } \mathrm{GV}^{\mathrm{b}}\end{array}$ & $\bullet$ & $\bullet$ & • & • & - & - & - & - & - & - & - & - & - & $\bullet$ \\
\hline & $\begin{array}{l}\text { Nivel de mezcla y suspensión en el } \\
\text { núcleo }\end{array}$ & • & - & • & $\bullet$ & 0 & 0 & - & - & - & - & - & - & - & - \\
\hline & Estratificación en tuberías horizontales & $\bullet$ & $\bullet$ & $\bullet$ & $\bullet$ & $\bullet$ & O & O & - & - & - & $\bullet$ & - & - & - \\
\hline & Mezcla y condensación en SRR & - & - & - & - & - & - & $\mathrm{O}$ & - & - & - & - & - & - & - \\
\hline & Rotura del sello del lazo & - & - & $\bullet$ & $\bullet$ & $\bullet$ & $\bullet$ & - & - & - & - & - & - & - & - \\
\hline & Estancamiento de agua? en el UP/CCFL & $\bullet$ & $\bullet$ & O & O & O & O & - & - & - & - & - & - & - & - \\
\hline & $\begin{array}{l}\text { Vaciado del núcleo y distribución de } \\
\text { caudal }\end{array}$ & • & $\bullet$ & 0 & 0 & 0 & 0 & - & - & - & - & - & - & - & - \\
\hline & Transferencia de calor núcleo cubierto & $\bullet$ & $\bullet$ & O & $\bullet$ & $\bullet$ & $\bullet$ & - & - & - & - & - & - & - & $\bullet$ \\
\hline & $\begin{array}{l}\text { Transf. de calor núcleo parcialmente } \\
\text { cubierto }\end{array}$ & $\bullet$ & $\bullet$ & 0 & $\bullet$ & • & 0 & - & - & - & - & - & - & 0 & - \\
\hline & Transf. calor primario GVs & $\bullet$ & $\bullet$ & $\bullet$ & $\bullet$ & $\bullet$ & O & - & - & - & - & - & - & - & $\bullet$ \\
\hline & Transf. calor secundario GVs & O & $\bullet$ & $\bullet$ & $\bullet$ & $\bullet$ & O & - & - & - & - & - & - & - & $\bullet$ \\
\hline & Termihidráulica del presionador & - & - & - & - & - & - & - & - & - & - & - & - & - & $\bullet$ \\
\hline & $\begin{array}{l}\text { Hidráulica de la tubería de } \\
\text { compensación }\end{array}$ & - & - & - & - & - & - & - & $=$ & - & - & - & - & - & $\bullet$ \\
\hline & comportamiento bombas 1 y 2 fases & - & - & - & - & - & - & - & - & - & - & - & - & - & O \\
\hline & Estructura de calor y pérdidas $^{\mathrm{c}}$ & $\bullet$ & $\bullet$ & $\bullet$ & O & O & O & - & - & - & - & - & - & - & \\
\hline & Efecto de incondensables & - & - & - & - & - & - & - & - & - & - & - & - & - & - \\
\hline & $\begin{array}{l}\text { Separación de fases en una junta } \mathrm{T} \text { y } \\
\text { efecto del caudal de fuga }\end{array}$ & - & - & • & 0 & 0 & 0 & - & - & - & - & - & - & - & - \\
\hline \multirow{3}{*}{ 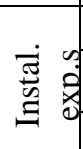 } & BETHSY & $\bullet$ & $\bullet$ & $\bullet$ & \multirow{3}{*}{\multicolumn{2}{|c|}{$\begin{array}{l}\text { a. } \\
\text { b. } \\
\text { c. }\end{array}$}} & \multirow{3}{*}{\multicolumn{8}{|c|}{$\begin{array}{ll} & \text { Volumétrica } \\
\text {. } & \text { Secundario } \\
\text { Problema para instalaciones a escala }\end{array}$}} & \\
\hline & LSTF & - & - & $\bullet$ & & & & & & & & & & & \\
\hline & SEMISCALE & - & - & $\bullet$ & & & & & & & & & & & \\
\hline
\end{tabular}

Tabla 5.2: Matriz de valoración del RELAP5/Mod3.0 para SBLOCAs en PWR. 
Con los resultados de los experimentos de efectos separados que aparecen en las matrices de valoración, tablas 5.1 y 5.2, se pretendía examinar la capacidad del código en las siguientes áreas:

1. Inestabilidad termohidráulica en sistemas con ligera ebullición y circulación natural.

2. Circulación natural en condiciones de bajo flujo.

3. Simulación de una rápida despresurización en el acumulador.

4. Correlaciones de flujos de calor crítico (CHF) y de secado.

5. Descargas a través de las válvulas de alivio y seguridad tras producirse una sobrecarga en la tubería.

6. Penetración de agua en la bajante de la vasija en accidentes cuyo iniciador es un LBLOCA.

7. Flujo contracorriente en las ramas calientes de un reactor PWR.

8. Condensación por contacto directo.

9. Fenómeno de limitación de flujo contracorriente en tubos y haces de tubos.

El primer estudio está basado en el transitorio "Edward's pipe" que es uno de los casos que se distribuyen para validar el RELAP5. En el segundo caso se ha modelado la instalación experimental del Royal Institute of technology (RIT) y se han ajustado algunos parámetros del fichero de entrada para que el error entre la predicción del código y los datos experimentales sea mínimo. En particular, en este segundo ejemplo se han estudiado dos de los experimentos que se realizaron en esta instalación experimental para determinar la respuesta del RELAP5 en la simulación del fenómeno de secado en diferentes condiciones de operación.

Ambos ejemplos de aplicación del método de búsqueda de parámetros elegidos se encuentran ampliamente estudiados por los usuarios del RELAP5 puesto que están incluidos en las matrices de validación del código RELAP5. El primero de los análisis consiste en el estudio de los fenómenos bifásicos que tienen lugar en una tubería al producirse una despresurización, en el cual se reproducen las características básicas de los procesos físicos que tienen lugar en una central nuclear al producirse un accidente de pérdida de refrigerante. En el segundo se trata de estudiar los fenómenos que se producen en las plantas cuando ante un accidente se produce el secado del núcleo, con la finalidad de detectar si el código calcula de manera adecuada los procesos de transferencia de calor. Ambos estudios se detallan en las secciones siguientes, y se incluyen como se ha mencionado anteriormente en las matrices de validación del código termohidráulico RELAP5. 


\subsection{Edward's pipe}

Una aplicación interesante del método de optimización de los parámetros en un modelo de RELAP5 es la comprobación de que el método es capaz de encontrar el valor más adecuado para los parámetros del fichero de entrada utilizando como referencia la salida proporcionada por el código para unos determinados valores de los parámetros contaminada con una cierta cantidad de ruido. Esta situación trata de simular los valores que se miden experimentalmente en las instalaciones, como presión y temperatura, los cuales llevan asociados un cierto ruido.

\subsubsection{Descripción del modelo de RELAP5}

Para realizar esta comprobación se ha utilizado el problema del "Edwad's pipe" que simula la despresurización de una tubería horizontal que se encuentra inicialmente cerrada y llena de agua a una presión de $7 \mathrm{MPa}$ y a una temperatura de $502^{\circ} \mathrm{K}$ (IonescuBujor2000a; Ionescu-Bujor2000b). El transitorio de despresurización se inicia después de liberar uno de los extremos de la tubería, simulada mediante un componente PIPE, que es de todos los componentes hidrodinámicos implementados en el código RELAP5, el que más se ajusta a la geometría que se desea simular (INEL1995b). Este transitorio es interesante debido a que tienen lugar distintos fenómenos físicos que afectan al fluido dentro de la tubería a lo largo del transitorio. En concreto, lo que se intenta observar es el cambio del agua líquida del interior de la tubería a mezcla bifásica durante la despresurización, y este proceso simula las características básicas que se presentan en un accidente cuyo iniciador es una pérdida de refrigerante de un reactor (IonescuBujor2000a; Ionescu-Bujor2000b).

Debido a las características de este transitorio, en el cálculo del comportamiento de variables como la presión, temperatura y velocidades del líquido y del vapor a lo largo del transitorio, se requiere la utilización de los modelos hidrodinámicos completos del RELAP5 bifásicos y, por lo tanto, es un buen ejemplo sobre el que aplicar la metodología de búsqueda de parámetros. El modelo para RELAP5 del sistema hidrodinámico sobre el que se produce el transitorio se presenta en la figura 5.1, cuyo fichero de entrada para RELAP5 se presenta en el Apéndice 1. 


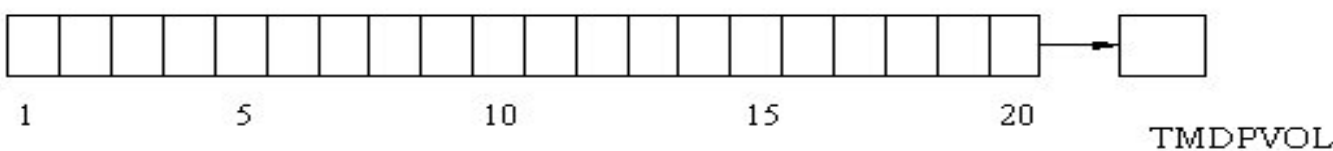

Figura 5.1: Nodalización del Edward's pipe para RELAP5.

El problema consta de una tubería representada por un componente hidrodinámico PIPE (INEL1995b; INEL1995d), constituida por 20 volúmenes que se encuentra unida mediante una junta, modelada por un componente SNGLJUN, a través del cual descarga directamente a la atmósfera que se encuentra simulada por un volumen TMDPVOL en el que se mantiene la presión atmosférica constante durante todo el transitorio y en el que solamente hay vapor. La suposición de que en las condiciones de contorno del problema que simula la atmósfera en lugar de aire se suponga vapor es una práctica habitual y aceptada por los usuarios del RELAP debido a las dificultades del código para simular los incondensables (Martorel12000a; Martorel12000b) y en ningún caso afectan significativamente a los procesos físicos que se producen en el interior de la tubería.

Acoplada a la tubería por la que pasa el fluido se encuentra una estructura de calor que modela las paredes de la misma y simula el intercambio de calor por convección entre las paredes y el agua que pasa por el interior de la tubería. Además del intercambio de calor con las paredes, al sistema se le aporta una determinada cantidad de calor debida al decaimiento de los productos de fisión. La potencia total que se aporta al sistema debido a este efecto durante el transitorio viene determinada por la cinética del problema. En este caso se calcula la potencia total a partir de la aproximación de cinética puntual, con una potencia total inicial de $10^{6} \mathrm{~W}$.

Como se ha mencionado anteriormente, este ejemplo es interesante porque reproduce las características básicas de los fenómenos físicos que se producen en un accidente con pérdida de refrigerante. Una característica de estos transitorios es la evacuación de calor a través de la rotura, lo cual afecta a la temperatura del fluido que pasa por el interior de la tubería disminuyendo su valor. Por esta razón, se ha considerado interesante tomar como variable de referencia la temperatura del líquido en el primer volumen de la PIPE que modela la tubería, es decir, el más alejado de la salida a la atmósfera. 


\subsubsection{Simulación y optimización de parámetros}

A partir del modelo suministrado con el RELAP5 se han realizado diversos análisis de sensibilidad cambiando el valor de algunos de los parámetros del fichero de entrada del problema con el fin de determinar aquellos para los que con pequeñas variaciones de su valor llevan a comportamientos finales del sistema diferentes, en concreto se ha buscado aquellos parámetros para los que la evolución de la temperatura del líquido difiere de la que se obtiene con el fichero de entrada original.

Con la finalidad de simplificar el problema de optimización se decidió elegir un único parámetro del modelo sobre el que realizar la búsqueda de aquellos que afectaban a la evolución de la temperatura del líquido. En particular, el análisis de sensibilidad puso de manifiesto que pequeñas variaciones en el valor inicial de la energía interna específica del líquido fijada como condición inicial en el interior de la tubería proporcionaban diferentes evoluciones con el tiempo para la temperatura del agua en el primer volumen del componente PIPE que la representa, lo cual sugería tomar como parámetro de búsqueda la energía interna específica del líquido en el instante inicial del transitorio.

En efecto, en el modelo suministrado con el RELAP5/Mod3.2, las condiciones iniciales de cada uno de los volúmenes que forman la tubería son las mismas, y vienen dadas por la presión y las energías internas específicas del líquido y del vapor. Sin embargo, en condiciones normales no se suele disponer de datos de energías internas, sino que, normalmente, las variables que se miden son la presión y la temperatura del fluido a partir de las cuales se obtienen las energías internas específicas del líquido y del vapor. Tanto en el proceso de toma de medidas de presión y temperatura como en el cálculo posterior de las energías internas, o incluso durante el proceso de asignar los valores en el fichero de entrada del modelo de RELAP pueden introducirse errores que alteren el valor de esta variable y, por tanto, produzcan una variación en la evolución de la temperatura del líquido que calcula el código.

Así, un ejemplo sencillo y adecuado para la comprobación de la metodología propuesta aplicada a un código termohidráulico consiste en la optimización del valor más adecuado de la energía interna específica del líquido que se debe especificar en el fichero de entrada del RELAP5/Mod3.2 para que la temperatura en uno de los volúmenes de la tubería, por ejemplo en este caso se ha considerado el primer volumen, sea lo más cercana posible a la de referencia.

Con el fin de comprobar si el método de búsqueda es capaz de encontrar los valores más adecuados para los parámetros, incluso en presencia de ruido, se ha simulado el transitorio durante 0.5 segundos, la evolución de la temperatura del agua se encuentra a su vez distorsionada con un ruido simulado mediante una variable aleatoria que se distribuye uniformemente en el intervalo $[-1,1]$ y que perturba el valor de temperatura 
real como mucho en un $5 \%$ del valor máximo que toma esta variable durante todo el transitorio. Esto es, se ha tomado como referencia el valor de la temperatura en cada instante de tiempo como

$$
\mathrm{T}(\mathrm{n})=\mathrm{T}_{\text {ref }}(\mathrm{n})+\operatorname{máx}_{\mathrm{n}}\left\{\mathrm{T}_{\text {ref }}(\mathrm{n})\right\} 0.05 \operatorname{rui}(\mathrm{n})
$$

donde $T_{\text {ref }}(n)$ es el valor, en el instante $n$, de la temperatura que se obtiene con el fichero de entrada suministrado con el RELAP5/Mod3.2 y rui(n) es el valor del ruido generado.

Con este planteamiento se ha realizado la minimización del error cometido en la simulación de la evolución de la temperatura del líquido tomando como parámetro a optimizar la energía interna específica del líquido. El valor de la energía interna específica se ha restringido al intervalo $\left[510^{5}, 10^{6}\right]$ puesto que fuera de este intervalo el RELAP no es capaz de encontrar una solución con sentido físico. En la tabla 5.3 se muestran las características del algoritmo MDS implementado para llevar a cabo la optimización.

En este caso, la variable sobre la que se calcula el error es un vector en el que se dispone el valor de la temperatura del agua en el primer volumen de la tubería para cada instante de tiempo. La cuantificación del error cometido en la simulación se ha calculado mediante la expresión del error cuadrático medio, o sea, la norma 2 del vector.

\begin{tabular}{ll}
\hline Características MDS & Valores \\
\hline Símplice inicial & $510^{5}$ \\
& $10^{6}$ \\
Paso contracción & 0.5 \\
Paso expansión & 2.0 \\
Iteraciones máximas & 100 \\
Evaluaciones máximas & 400 \\
Tolerancia & $1.010^{-7}$ \\
\hline
\end{tabular}

Tabla 5.3: Características del MDS en el Edward's pipe.

Utilizando el MDS descrito se llega al resultado óptimo que se presenta en la tabla 5.4, junto con el número de evaluaciones necesarias para alcanzar este resultado. Además, en la misma tabla se presenta el valor de la variable para generar la evolución de la temperatura tomada como referencia.

\begin{tabular}{lll}
\hline & Referencia & Óptimo \\
\hline Energía Interna(J/Kg) & $9.010^{5}$ & $9.9710^{5}$ \\
Número de evaluaciones de la función objetivo & & 102 \\
\hline
\end{tabular}

Tabla 5.4: Resultado de la optimización. 
En la figura 5.2 se muestra la evolución de la temperatura del líquido en este caso de aplicación. En la figura se observa la robustez del método frente al ruido, puesto que ha encontrado el valor del parámetro más adecuado para de la energía interna específica que debe utilizarse para reproducir la temperatura de referencia sin que se vea afectada por el ruido que la distorsiona.

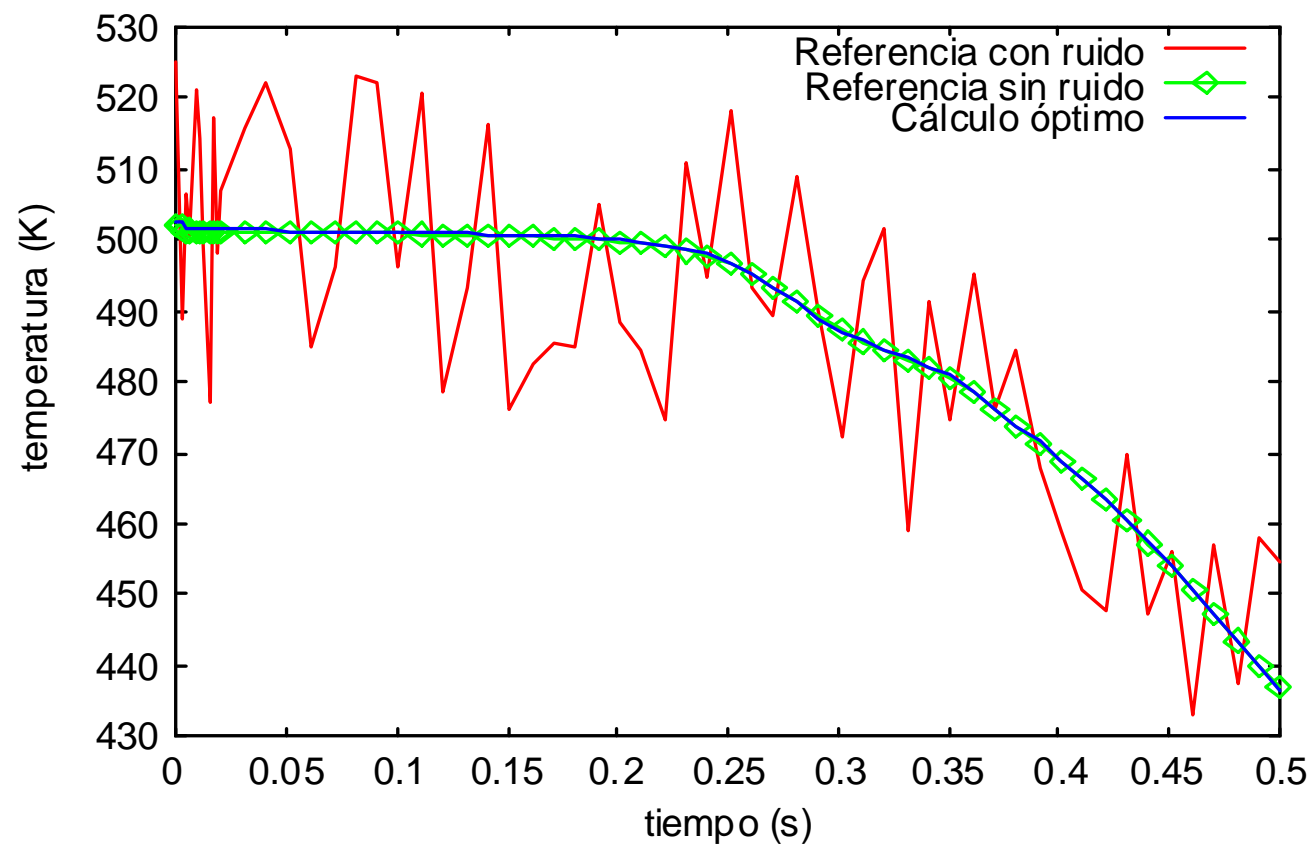

Figura 5.2: Evolución de la temperatura en el problema Edward's pipe.

\subsection{Royal Institute of Technology (RIT)}

El segundo de los ejemplos utilizados para mostrar el funcionamiento de la metodología de estimación de parámetros de códigos termohidráulicos consiste en la revisión de los modelos de planta utilizados en dos experimentos incluidos en el proceso de validación del código RELAP5/Mod3, con la finalidad de explicar algunas de las discrepancias que se manifestaron en el análisis de los resultados obtenidos del estudio realizado (Nilsson1993) para determinar la capacidad del RELAP5/Mod3.2 en cuanto al cálculo del flujo de calor crítico (CHF) y las correlaciones de transferencia de calor, experimento desarrollado en la instalación experimental del Royal Institute of Technology (RIT) (Nilsson1993) de Estocolmo.

La instalación RIT ha sido utilizada dentro del programa International Thermalhydraulic Code Assesment and Applications Program (ICAP), con el fin de realizar una valoración cuantitativa de la precisión de los códigos termohidráulicos tanto TRACPWR como RELAP5. Los experimentos llevados a cabo en esta instalación pretendían 
estudiar la simulación del proceso de transferencia de calor después del secado que realizan los códigos.

La finalidad de los programas como el ICAP es la revisión constante de los modelos incluidos en los códigos termohidráulicos, y su sustitución por modelos que se ajusten más a la realidad. Así, en la versión RELAP5/Mod3 se introdujo una variación en el método de cálculo del flujo crítico de calor, en principio mejor que la de la versión Mod2. Para comprobar la mayor capacidad del modelo se tomaron como referencia los experimentos de la instalación RIT y se hizo una comparación de los resultados de las simulaciones con ambas versiones de código, cuyos resultados y conclusiones se presentan en la referencia (Nilsson1993). Como conclusión general del experimento se valoró como razonable el cálculo del CHF, sin embargo, la predicción de la localización del punto en el que se produce este efecto no era en general conservadora. En efecto, en la mayoría de los casos analizados el perfil axial de la temperatura de la pared señalaba que el punto en el que se produce el secado estaba más arriba de lo que se había medido en la instalación. Además, con respecto a los valores de la temperatura de la pared calentada, los valores calculados por el código eran inferiores a los experimentales, sobre todo en la zona posterior a producirse el secado.

Las discrepancias observadas en los experimentos realizados pueden ser debidas tanto a problemas en las correlaciones internas que utiliza el RELAP5 para el cálculo de estos fenómenos como a desajustes en los parámetros del modelo de planta a partir del cual se ha realizado la simulación.

Así, además de la mejora en los modelos de cálculo de los códigos, existen otros aspectos que influyen en el ajuste de los resultados de la simulación para un modelo determinado, como son la elección de unas condiciones de contorno y unas condiciones iniciales para el modelo adecuadas (INEL1995b; INEL1995d), así como una buena elección de los parámetros de las correlaciones que intervienen en el modelo. Todo esto debe reflejarse en el fichero de entrada del código.

En este ejemplo se aplica la metodología de optimización con la finalidad de estimar algunos de los parámetros del modelo del sistema diseñado para esta instalación de manera que la respuesta proporcionada por el RELAP5/Mod3 sea lo más ajustada posible a los datos experimentales de temperatura, que se tomarán como referencia. El ejercicio se repetirá sobre dos experimentos, RIT136 y RIT139. 


\subsubsection{Descripción de la instalación}

La instalación experimental RIT en la cual se realizaron los experimentos está diseñada para trabajar a una presión de 250 bar y todas las zonas del lazo que están en contacto con el agua son de acero inoxidable. En la figura 5.3 se muestra un esquema simplificado de la instalación.

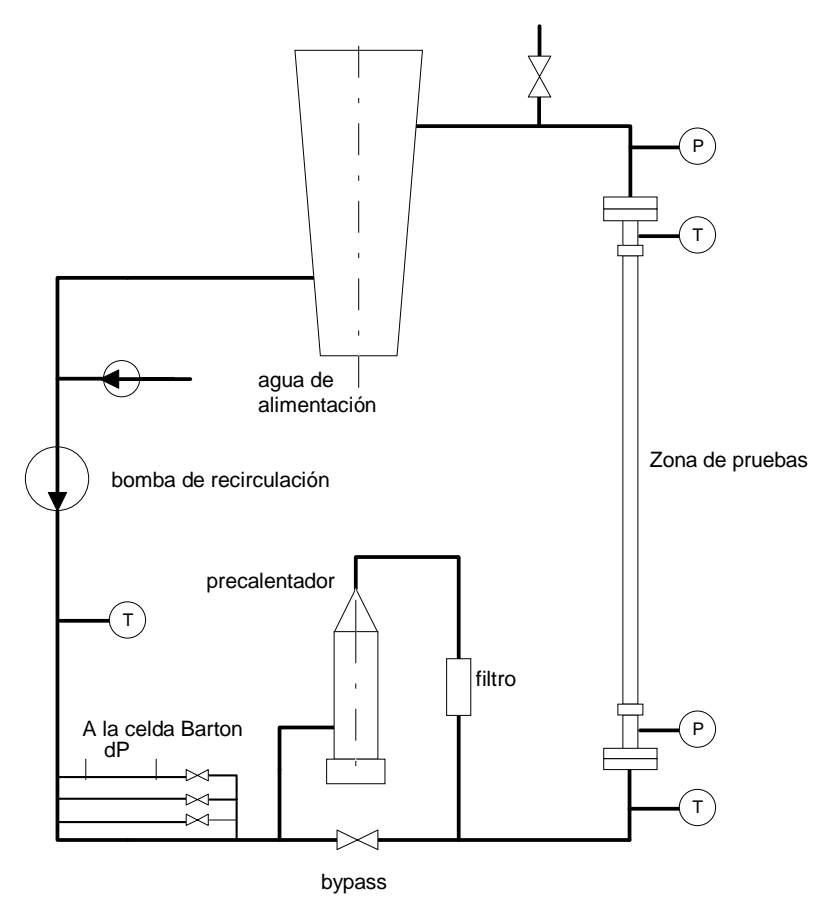

Figura 5.3: Esquema simplificado de la Instalación RIT.

Antes de entrar en la zona donde se realizan los experimentos el agua pasa a través de un precalentador donde se ajusta la temperatura de entrada del agua y posteriormente pasa por un filtro. Después de pasar por la zona de pruebas, la mezcla bifásica pasa por el condensador y después es impulsada mediante una bomba de recirculación hacia la entrada de la zona de pruebas con la suficiente presión para conseguir un funcionamiento seguro y estable del lazo. El caudalímetro que mide la cantidad de agua consiste en cuatro tubos de $1 \mathrm{~m}$ de longitud, en uno de ellos se mide la caída de presión mediante una celda Barton y proporciona así una medida del caudal de agua que pasa a través de los tubos. Este método de medida proporciona valores con una desviación menor del $1 \%$ en todo el rango de aplicación. Las celdas Barton se utilizan también para medir la presión en el lazo conectadas con los sensores de presión que se encuentran localizados justo debajo y arriba de la zona de pruebas. Este método de medida de la presión tiene una precisión de un $0.1 \%$. En cuanto a las medidas de temperaturas, como puede observarse en la figura 5.3, existen tres puntos de medida: antes del caudalímetro, antes de entrar en la sección de pruebas y a su salida. La temperatura se mide mediante termopares montados en unas cavidades de $100 \mathrm{~mm}$ de espesor y $3 \mathrm{~mm}$ de diámetro interno, proporcionando una precisión en las medidas de temperatura de $0.5 \mathrm{~K}$. 
El valor de la potencia que genera la instalación se obtiene a partir de la medida de la corriente eléctrica y del voltaje en la zona de pruebas. El voltaje se mide con un voltímetro cuya precisión era de $0.25 \%$ y la corriente se mide a partir del voltaje a través de una junta calibrada con una precisión del $0.25 \%$.

Las comprobaciones de la precisión global de la instrumentación se obtuvieron mediante la realización de balances de calor en una sola fase, en función de la aportación de calor y del incremento de entalpía del agua, obligando a que el error en el balance energético fuese menor de un $1 \%$.

Los experimentos que después fueros simulados con RELAP5 se realizaron sobre un tubo de $7 \mathrm{~m}$ de longitud con un diámetro interior de $14.9 \mathrm{~mm}$ y un diámetro exterior de $14.0 \mathrm{~mm}$, esta zona es la denominada zona de pruebas en la figura 5.3. En la pared exterior de este tubo se encuentran montados los termopares, debidamente calibrados, que miden la temperatura a lo largo del tubo, tal como se muestra en la figura 5.4. Para cada uno de los experimentos se obtuvo la distribución axial de la temperatura en la pared interior del tubo a partir de los datos recogidos por los termopares.

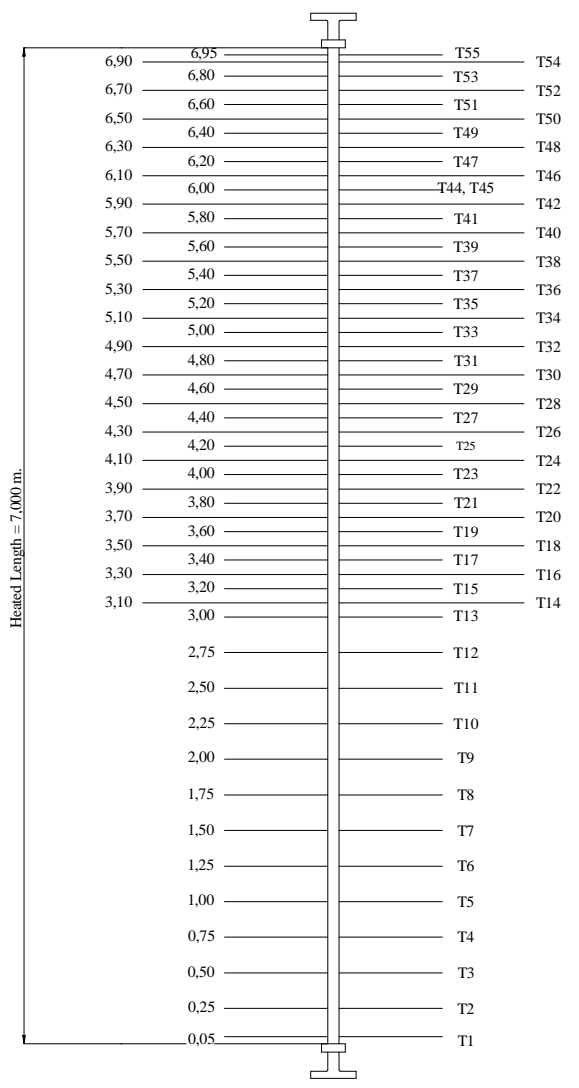

Figura 5.4: Localización de los termopares en la zona calentada. 
Para la comparación de los resultados obtenidos con las dos versiones del código termohidráulico RELAP5, Mod2 y Mod3, se simularon una serie de experimentos llevados a cabo en la instalación RIT, en los cuales varían el valor de los parámetros de partida. En la tabla 5.5 se muestran los rangos de los parámetros para cada simulación.

\begin{tabular}{ccccccc}
\hline Caso & $\begin{array}{c}\text { Presión } \\
(\mathrm{MPa})\end{array}$ & $\begin{array}{c}\text { Caudal } \\
\left(\mathrm{kg} / \mathrm{m}^{2} \mathrm{~s}\right)\end{array}$ & $\begin{array}{c}\text { Temp. entrada } \\
\left({ }^{\circ} \mathrm{K}\right)\end{array}$ & $\begin{array}{c}\text { Flujo de calor } \\
\left(\mathrm{kW} / \mathrm{m}^{2}\right)\end{array}$ & $\begin{array}{c}\text { Localización } \\
\text { del CHF }(\mathrm{m})\end{array}$ & $\begin{array}{c}\text { Calidad del vapor a } \\
\text { la salida }\end{array}$ \\
\hline$* 136$ & 13.99 & 1977.0 & 599.6 & 509 & 5.55 & 0.384 \\
$* 139$ & 14.00 & 1970.5 & 600.0 & 757 & 2.88 & 0.608 \\
147 & 14.00 & 1494.3 & 600.0 & 704 & 2.88 & 0.761 \\
154 & 13.98 & 1.006 .6 & 600.4 & 552 & 3.15 & 0.896 \\
161 & 13.99 & 503.2 & 600.2 & 405 & 2.88 & 1.347 \\
220 & 10.00 & 1981.2 & 574.4 & 551 & $*$ & 0.324 \\
221 & 10.01 & 1973.8 & 574.2 & 665 & 5.55 & 0.434 \\
224 & 10.02 & 1990.3 & 573.4 & 860 & 4.25 & 0.567 \\
232 & 10.00 & 1499.8 & 573.8 & 758 & 4.15 & 0.673 \\
238 & 10.01 & 997.3 & 572.2 & 556 & 4.85 & 0.740 \\
244 & 9.98 & 502.0 & 573.0 & 405 & 5.05 & 1.099 \\
259 & 6.99 & 1989.3 & 549.0 & 870 & 6.05 & 0.510 \\
261 & 7.02 & 1988.2 & 548.4 & 1053 & 4.65 & 0.624 \\
264 & 6.99 & 1500.2 & 547.8 & 766 & 6.05 & 0.598 \\
269 & 7.01 & 995.4 & 547.8 & 564 & $*$ & 0.667 \\
270 & 7.02 & 996.5 & 547.2 & 660 & 5.85 & 0.785 \\
272 & 7.02 & 999.6 & 547.4 & 815 & 4.55 & 0.977 \\
275 & 7.04 & 500.9 & 547.2 & 410 & 6.45 & 0.980 \\
315 & 3.01 & 1986.3 & 498.0 & 873 & 5.95 & 0.436 \\
317 & 3.03 & 1987.3 & 497.2 & 1058 & 5.05 & 0.532 \\
320 & 2.99 & 1499.3 & 497.6 & 769 & 6.05 & 0.513 \\
325 & 3.01 & 1110.6 & 496.2 & 561 & $*$ & 0.554 \\
327 & 3.00 & 1005.6 & 497.2 & 667 & 6.05 & 0.669 \\
331 & 3.00 & 497.2 & 498.6 & 413 & $*$ & 0.847 \\
332 & 3.00 & 496.4 & 497.8 & 464 & 6.25 & 0.954 \\
\hline & & & & \\
& & & & & & \\
\end{tabular}

*Experimentos considerados en este estudio

Tabla 5.5: Rango de valores cubierto en las simulaciones.

\subsubsection{Descripción del modelo de RELAP5}

Puesto que el fenómeno físico que se pretende simular tiene lugar únicamente en la llamada zona de pruebas, el fichero de entrada del RELAP utilizado para realizar las simulaciones consiste básicamente en el modelo de este tubo, siendo el resto del lazo sustituido por unas condiciones de contorno adecuadas. Así, para el modelo del tubo se ha utilizado un volumen hidródinámico definido en el RELAP como PIPE, el cual está a su vez dividido en 47 volúmenes con las longitudes adecuadas para que cada punto de medida de la temperatura de la pared interna del experimento coincida con el punto en el cual RELAP calcula este parámetro. Esta PIPE está acoplada a una estructura de calor 
que simula el espesor de la pared del tubo que se supone aislado por la parte exterior, y en contacto con el fluido de cada uno de los volúmenes que componen la PIPE en la parte interior. El cálculo de la temperatura en la pared del tubo se realiza en la estructura de calor a partir de un mallado radial del espesor del tubo en que se calcula la temperatura en cada punto hasta llegar a la pared interior.

En cuanto a las condiciones de contorno, la entrada de la tubería se ha simulado mediante un TMDPVOL que fija las condiciones de presión y temperatura del agua en esta zona y de una TMDPJUN que mantiene fijo el caudal de agua de entrada. La parte superior de la tubería se ha representado por un TMDPVOL que fija las condiciones exteriores y se encuentra unido mediante una SNGLJUN a la tubería. Los valores de las condiciones de contorno dependen del experimento a simular según muestra la tabla 5.5.

La nodalización de la instalación RIT utilizada en las simulaciones se presenta en la figura 5.5, donde aparecen los volúmenes termohidráulicos que la forman, y la estructura de calor está acoplada en la PIPE. Un fichero de entrada completo para RELAP se presenta en el Apéndice 2 de la tesis.

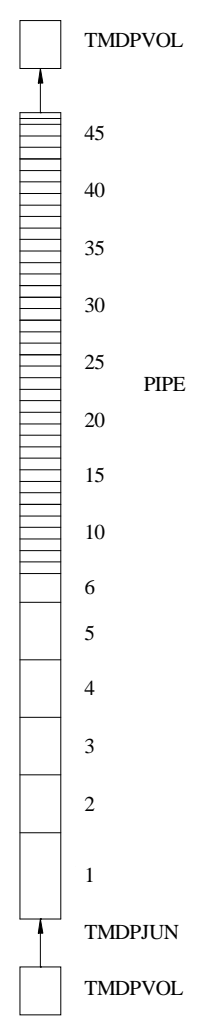

Figura 5.5: Nodalización de la instalación RIT para RELAP5. 


\subsubsection{Experimentos simulados con optimización de parámetros}

Los resultados de las simulaciones dependen en gran medida, además de los modelos de cálculo utilizados por el código, de los efectos de usuario introducidos en el fichero de entrada. Una de las incertidumbres que se pueden plantear en la confección de un modelo para un sistema determinado es la elección de unas condiciones de contorno adecuadas. Para cada uno de los experimentos, RIT136 y RIT139, se ha calculado mediante RELAP la distribución axial de la temperatura de la pared en un cierto instante de tiempo y se ha comparado con las medidas experimentales (Nilsson1993), observando que existen claras diferencias en los resultados.

En los ficheros de entrada para la simulación mediante RELAP5 de los experimentos RIT se han supuesto las condiciones presentadas en la tabla 5.5; no obstante existen algunas variables, como la presión a la salida de la tubería, cuyo valor podría diferir de los propuestos. Esto implica la necesidad de realizar una búsqueda y optimización del valor más adecuado para estos parámetros, con el fin de que el error obtenido con los resultados de la simulación y en comparación con los datos experimentales sea el mínimo posible. Este problema de optimización, como en el caso del "Edward's pipe", se resolverá utilizando el método MDS.

\subsubsection{Experimento RIT 136}

Para el estudio de este experimento se parte del modelo para RELAP proporcionado en la referencia (Nilsson1993), y que se presenta en el Apéndice 2 de esta tesis. Realizando la simulación con este fichero de entrada se ha obtenido el perfil axial de temperaturas que hemos denominado caso base, y que difiere bastante del perfil experimental, tal como puede observarse en la figura 5.6.

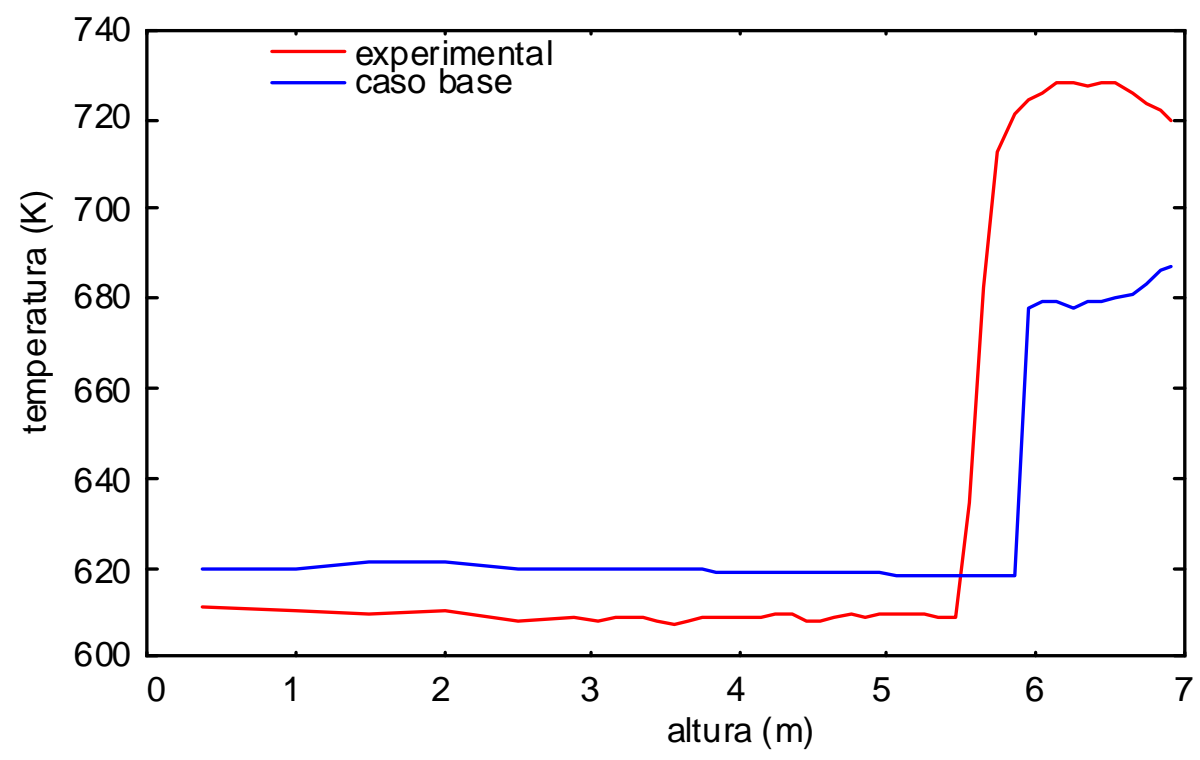

Figura 5.6: Comparación entre las medidas experimentales y el caso base para RIT136. 
Tras la realización de los análisis de sensibilidad para buscar y determinar los parámetros que más influyen en el perfil de temperaturas, se detectó que la presión a la salida de la tubería podría ser uno de ellos. Por otra parte, en el fichero de entrada, a la presión en la parte inferior y superior de la tubería se les ha asignado el mismo valor, lo cual no será correcto en la realidad puesto que la tubería es vertical. Por esta razón, en la primera de las optimizaciones llevadas a cabo se abordó el problema de encontrar la presión a la salida del tubo que mejor reprodujese el perfil de temperaturas experimental (mínimo error).

La presión de salida, $\mathrm{P}_{\mathrm{s}}$, con la que el RELAP5 realiza la simulación, se relaciona con la variable $\mathrm{x}_{1}$ utilizada por el MDS en el procedimiento de optimización de la siguiente manera

$\mathrm{P}_{\mathrm{s}}=\mathrm{P}_{\mathrm{e}}-\mathrm{x}_{1} 10^{6}$

donde $\mathrm{P}_{\mathrm{e}}$ es la presión a la entrada de la tubería, que en este caso permanece constante e igual a 13.99 $\mathrm{MPa}$, valor obtenido de los datos de la tabla 5.5.

Para la estimación de la presión de salida se ha tenido en cuenta el hecho de que, al igual que las otras variables, cada valor de la presión de salida determina comportamiento del fluido en la tubería, por lo que en el proceso de búsqueda se ha tenido que limitar el valor de la variable a un cierto intervalo de variación, de forma que se tengan valores de la presión de salida que permitan al RELAP5 llegar a una solución con significado físico. En particular la variable, $\mathrm{x}_{1}$, ha sido limitada dentro del intervalo $[0.0,6.0]$. Para implementar esta limitación en el algoritmo MDS se ha hecho una primera comprobación del valor de la variable $\mathrm{x}_{1}$, si este valor está fuera del intervalo de variación se le asigna un error suficientemente grande y sólo se hace el cálculo del error real ejecutando el RELAP5 si la variable está dentro del intervalo de variación permitido. El MDS es un algoritmo convergente para restricciones de este tipo (Torczon2000a) y por tanto se garantiza un buen funcionamiento del método en este tipo de problemas. Los valores que definen el símplice inicial del método MDS, el valor óptimo alcanzado de $\mathrm{x}_{1} \mathrm{y}$ la correspondiente presión de salida que define para este problema se presentan en la tabla 5.6.

\begin{tabular}{llll}
\hline & Inicial & Referencia & Óptimo \\
\hline $\mathrm{x}_{1}$ & $(0.0,3.0)$ & 0.0 & 0.231 \\
Presión de salida (MPa) & 13.99 & 13.99 & 13.758 \\
& 10.99 & & \\
Evaluaciones de la función objetivo & & & 50 \\
\hline
\end{tabular}

Tabla 5.6: Valores de la optimización del RIT136 para 1 parámetro. 
En la figura 5.7 se compara el perfil de temperaturas calculado mediante RELAP5 para estas nuevas condiciones de contorno con los datos experimentales y con el caso base. Como se puede observar en esta figura, la posición en la que se produce el secado sigue sin ajustarse a los datos experimentales; de hecho, en la optimización de la presión de salida la curva de temperaturas obtenida únicamente presenta una pequeña mejora respecto del caso base en el tramo más alto de la tubería, donde las temperaturas son ligeramente superiores, sin embargo en la zona anterior a este punto el valor calculado por el código sigue siendo superior al medido experimentalmente.

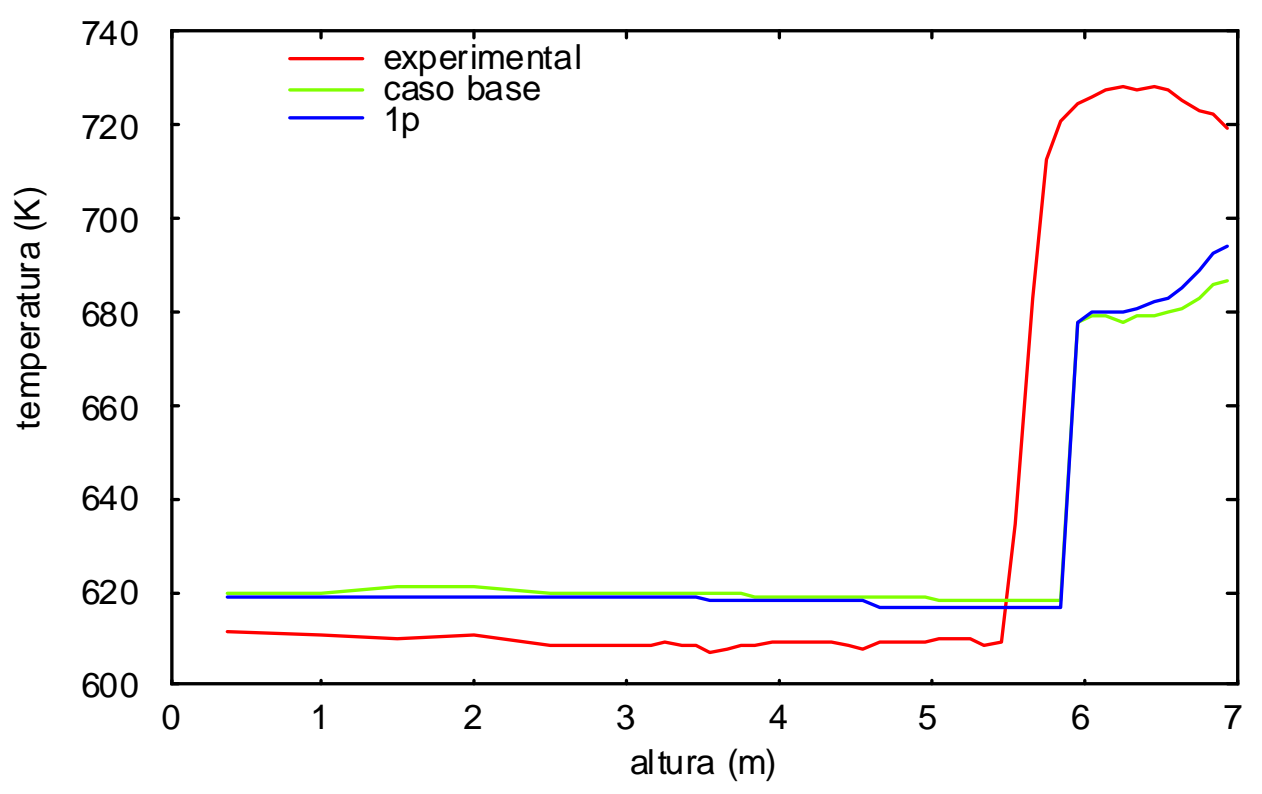

Figura 5.7: Perfil axial de temperatura. Optimización 1 parámetro RIT136.

En vista de los resultados obtenidos se plantea la necesidad de introducir nuevas variables en el proceso de optimización. Debido a que la temperatura antes de producirse el secado se mantiene constante y con un valor de unos $10^{\circ} \mathrm{K}$ superior a la experimental, se ha creído conveniente la optimización de alguno de los parámetros fijados como condiciones de contorno a la entrada de la tubería, esto es, presión, temperatura o caudal de agua de entrada a la zona calentada.

En primer lugar se aborda el problema de buscar conjuntamente los valores de las presiones a la entrada y salida de la tubería con el fin de encontrar la pareja de valores que hagan mínimo el error cometido en la simulación. En este caso el vector de variables de búsqueda del MDS viene definido por la variable $\mathrm{x}_{1}$, relacionada con el valor de la presión de entrada de la tubería y la $\mathrm{x}_{2}$ utilizada para obtener el valor de la presión a la salida de la siguiente forma

$\mathrm{P}_{\mathrm{e}}=\mathrm{x}_{1} 10^{7}$

$\mathrm{P}_{\mathrm{s}}=\left(\mathrm{x}_{1} 10-\mathrm{x}_{2}\right) 10^{6}$. 
Las relaciones anteriores entre las variables a optimizar y el valor real de las presiones se han tomado de este modo debido a la necesidad de escalar las variables de búsqueda que utiliza el MDS, de manera que al realizar las expansiones y contracciones del método no se produzcan situaciones que deformen mucho el símplice y poder llegar a soluciones razonables del problema.

Puesto que las variables de este problema de optimización definen las presiones en las condiciones de contorno del sistema físico del modelo de entrada del RELAP5, es necesario que se mantengan dentro de unos valores que tengan significado físico, como ocurría en el caso anterior. En este caso las variables se han limitado dentro de los intervalos, $[0.8,3.4]$ para la variable $\mathrm{x}_{1} \mathrm{y}[0,6]$ para $\mathrm{x}_{2}$.

Los valores que definen el símplice inicial del MDS se han elegido como se recomienda en la referencia (Torczon1989). Los valores del símplice inicial, el valor óptimo alcanzado de $\mathrm{x}_{1} \mathrm{y}_{2}$, así como las correspondientes presiones a la entrada y a la salida de la tubería para este problema se presentan en la tabla 5.7.

\begin{tabular}{llll}
\hline & Inicial & Referencia & Óptimo \\
\hline$\left(\mathrm{x}_{1}, \mathrm{x}_{2}\right)$ & $(1.399,0.0)$ & $(0.0,0.0)$ & $(1.427,0.441)$ \\
& $(2.399,0.0)$ & & \\
& $(1.399,1.0)$ & & \\
Presión de entrada (MPa) & 13.99 & 13.99 & 14.27 \\
& 23.99 & & \\
& 13.99 & & 13.83 \\
Presión de salida (MPa) & 13.99 & 13.99 & \\
& 23.99 & & 108 \\
Número de evaluaciones de la & 14.99 & & \\
función objetivo & & & \\
\hline
\end{tabular}

Tabla 5.7: Valores de la optimización del RIT136 para 2 parámetros.

En la figura 5.8 se compara la distribución axial de temperatura calculada para los valores optimizados de las presiones de entrada y salida de la tubería, con la distribución del caso base y con los datos experimentales. En este caso se observa que en la zona anterior al secado la temperatura de la pared está más cercana a la experimental, aunque sigue siendo mayor. Sin embargo, el punto en el que se produce el secado está más lejos del experimental de lo que predice el caso base, aunque las temperaturas que alcanza después de producirse el secado también se ajustan más a los valores que se midieron en la instalación que los del caso base. 


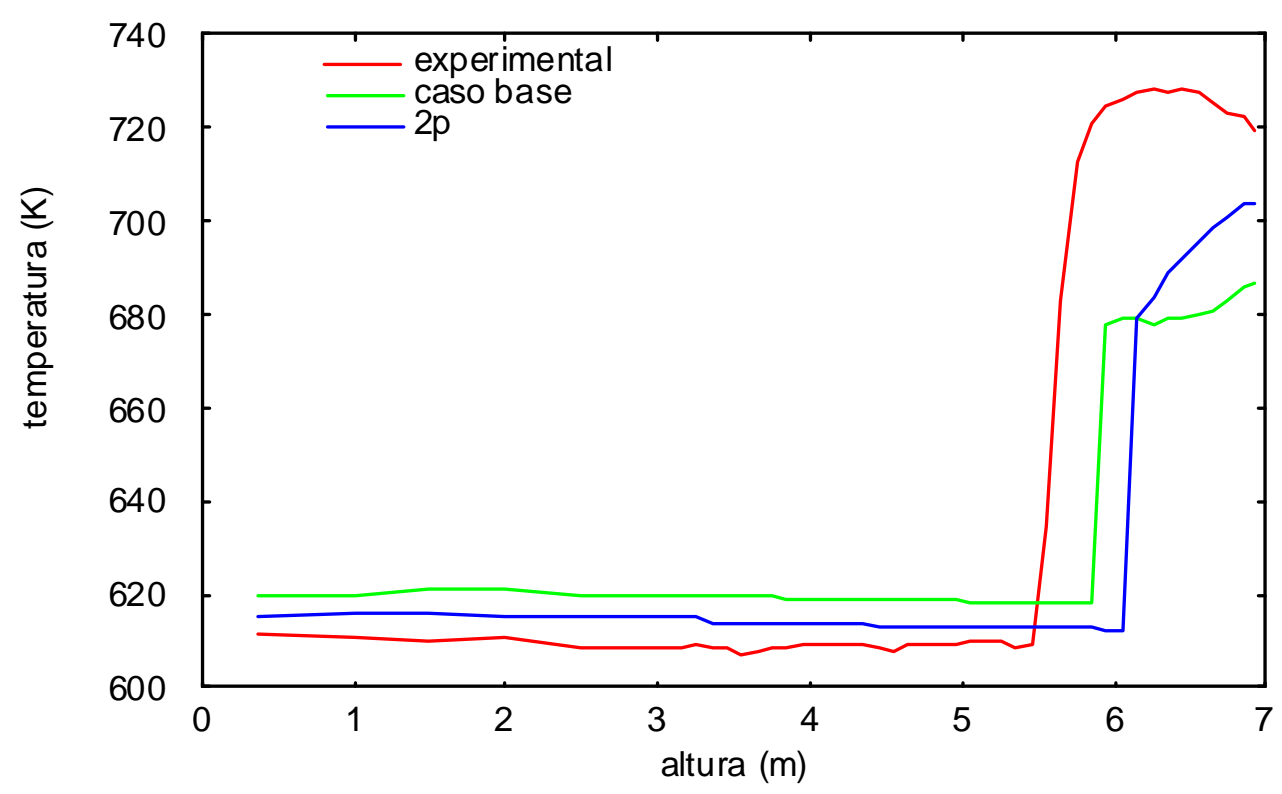

Figura 5.8: Perfil axial de temperatura. Optimización 2 parámetros RIT136.

Con el fin de mejorar los resultados obtenidos para el punto en el que produce el fenómeno de secado se introduce otro parámetro del sistema como variable de optimización. En este caso, se ha considerado el caudal de agua de entrada en la zona inferior de la tubería como tercera variable del problema a optimizar. De este modo, ahora se consideran las presiones a la entrada y a la salida de la tubería, $\mathrm{P}_{\mathrm{e}}$ y $\mathrm{P}_{\mathrm{s}}$, y el caudal de agua de entrada, $\mathrm{m}_{\mathrm{e}}$. De nuevo se escala el problema relacionando los parámetros señalados del modelo con las variables a optimizar con el MDS mediante las expresiones siguientes

$\mathrm{P}_{\mathrm{e}}=\mathrm{x}_{1} 10^{7}$

$\mathrm{P}_{\mathrm{s}}=\left(\mathrm{x}_{1} 10-\mathrm{x}_{2}\right) 10^{6}$,

$\mathrm{m}_{\mathrm{e}}=\mathrm{x}_{3} 10^{-1}$.

En cuanto a las restricciones que debe cumplir cada una de estas variables, para las dos presiones se han establecido los mismos criterios de restricción planteados en el caso anterior de optimización, mientras que sobre el caudal de agua de entrada no se ha impuesto ninguna restricción. El caudal de agua se puede considerar un número real sin restricciones porque a partir de los puntos iniciales, aunque haya expansiones y contracciones del símplice, no llegan a obtenerse valores para esta variable que hagan que el sistema pierda su significado físico; incluso si la variable resulta ser negativa el modelo de RELAP5 entiende que es un caudal de salida desde la tubería y por tanto realizaría la simulación. 
Los valores del símplice inicial utilizado para este nuevo problema, el valor óptimo alcanzado de $\mathrm{x}_{1}, \mathrm{x}_{2} \mathrm{y} \mathrm{x}_{3}$, así como las correspondientes presiones a la entrada y a la salida de la tubería y el caudal de agua de entrada para el problema se presentan en la tabla 5.8.

\begin{tabular}{llll}
\hline & Inicial & Referencia & Óptimo \\
\hline$\left(\mathrm{x}_{1}, \mathrm{x}_{2}, \mathrm{X}_{3}\right)$ & $(1.399,0.0,3.446)$ & $(1.399,0.0,3.446)$ & $(1.274,0.275,2.825)$ \\
& $(0.399,0.0,3.446)$ & & \\
& $(1.399,1.0,3.446)$ & & 12.734 \\
& $(1.399,0.0,4.446)$ & & \\
Presión de entrada (MPa) & 13.99 & 13.99 & 12.219 \\
& 3.99 & & \\
& 13.99 & & \\
Presión de salida (MPa) & 13.99 & 13.99 & 0.282 \\
& 13.99 & & \\
Caudal de entrada (Kg /s) & 13.99 & 0.3446 & 304 \\
& 14.99 & & \\
Número de evaluaciones de la & 13.99 & & \\
función objetivo & 0.3446 & & \\
\hline
\end{tabular}

Tabla 5.8: Valores de la optimización del RIT136 para 3 parámetros.

Como se puede observar los tres valores son ligeramente inferiores a los valores del caso base. Utilizando los nuevos valores de estos parámetros en el fichero de entrada del RELAP5/Mod 3.2 el perfil axial de temperatura que calcula el código se presenta en la figura 5.9, junto con el obtenido para el caso base y el experimental. Se observa la mejora considerable en la simulación del perfil axial de temperatura tras la optimización con tres parámetros. En este caso la temperatura antes de producirse el secado prácticamente coincide con la experimental, y además el punto en el que se produce este fenómeno coincide también con el experimental. Sin embargo, a partir de este punto todavía existe una pequeña diferencia entre los valores de temperatura calculados por el código y los experimentales debida, según (Nilsson1993), a los modelos de transferencia de calor implementados en el RELAP5/Mod3 que actúan después de producirse el secado. 


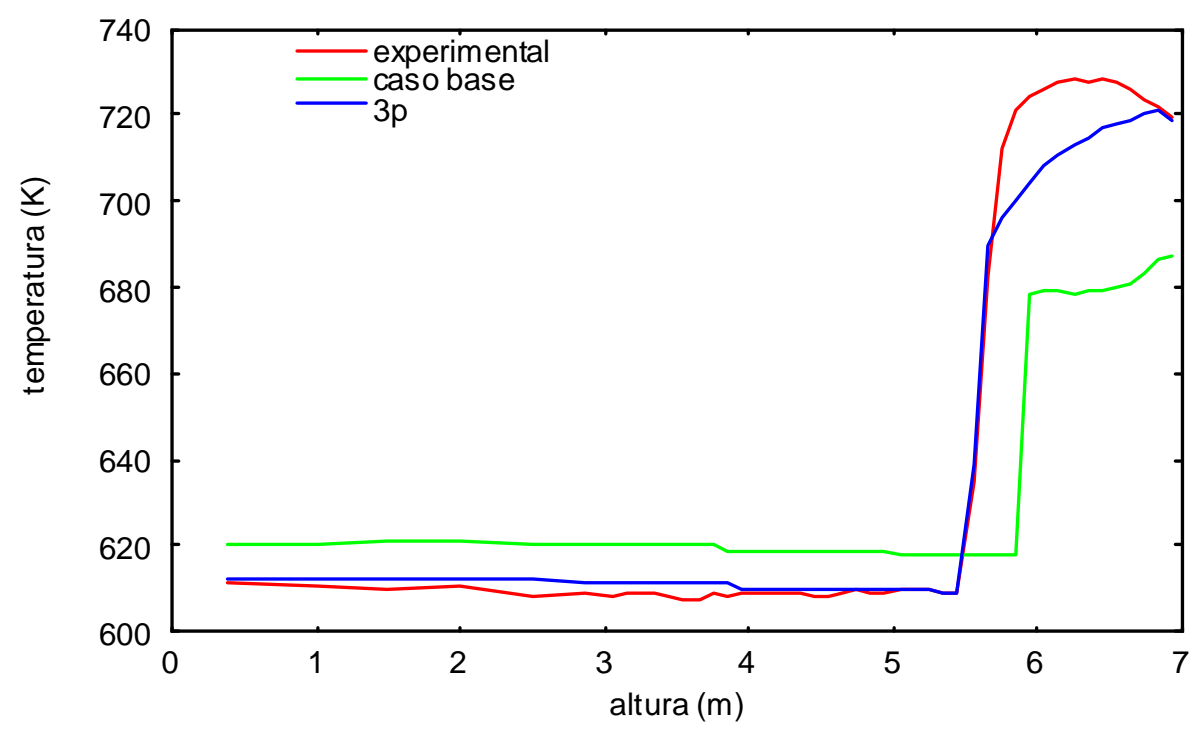

Figura 5.9: Perfil axial de temperatura. Optimización con 3 parámetros.

\subsubsection{Experimento RIT 139}

Con el fin de asegurar que la metodología de búsqueda de parámetros aplicada a la mejora de los modelos de RELAP5 es general y que el ejemplo anterior no supone un caso aislado de validez de esta metodología, se abordó la mejora del modelo del experimento 139.

El objetivo del experimento 139 llevado a cabo sobre la instalación RIT pretende simular también el fenómeno de secado de la tubería vertical a la que se le aplica calor, pero bajo unas condiciones ligeramente diferentes. Los valores de las variables más importantes se muestran en la tabla 5.5. En este caso se aplica calor durante 20 segundos, momento en el que se toman las medidas de temperatura en la pared de la tubería calentada.

También para el experimento 139 realizado en la instalación (RIT139) los resultados calculados con el RELAP5 difieren en gran medida de las mediciones realizadas en la instalación según se puede apreciar en la figura 5.10. En la realización del fichero de entrada de RELAP se han seguido las directrices del caso anterior, tomado los valores de las variables que señala la tabla 5.5 para este experimento. En concreto se ha fijado una presión de $14.00 \mathrm{Mpa}$, valor que se ha tomado tanto a la entrada como a la salida de la tubería. 


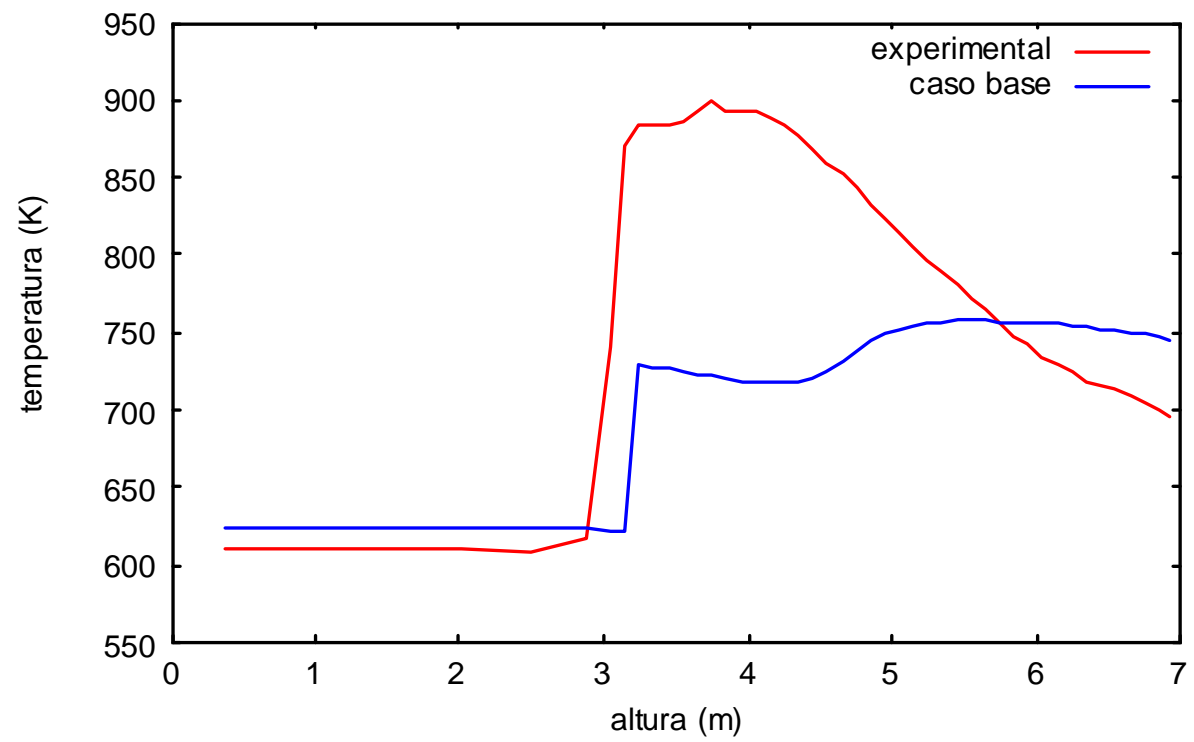

Figura 5.10: Comparación entre las medidas experimentales y el caso base para RIT139.

En este caso, tal como se puede observar en la figura 5.10, el punto en el que se produce el secado está desplazado y las temperaturas no alcanzan los valores dados en el experimento. Esta situación ya se producía en el caso anterior por lo que se consideró conveniente que la búsqueda y optimización se realizara de forma análoga a la anterior. Por tanto, en primer lugar se planteó la posibilidad de ajustar la distribución axial de la temperatura realizando una búsqueda del valor de la presión en la parte superior de la tubería que pudiera mejorar los cálculos realizados por el RELAP5.

Como en el caso anterior, el método de búsqueda utilizado es el MDS y la variable de búsqueda del método es la diferencia escalada entre la presión a la entrada y a la salida de la tubería. Así, la relación viene dada por la expresión

$\mathrm{P}_{\mathrm{s}}=\left(\mathrm{P}_{\mathrm{e}}-\mathrm{x}_{1}\right) 10^{6}$

siendo, $\mathrm{P}_{\mathrm{s}}$ el valor de la presión en la parte superior de la tubería (salida), $\mathrm{P}_{\mathrm{e}}$ la presión en la parte inferior de la tubería (entrada), y $\mathrm{x}_{1}$ el valor de la variable que se va a optimizar. Análogamente al caso anterior la variable $\mathrm{x}_{1}$ se encuentra limitada dentro de un intervalo que asegura que el RELAP5 encuentra una solución. El símplice inicial, los valores de referencia y los resultados de esta optimización se presentan en la tabla 5.9. 


\begin{tabular}{llll}
\hline & Inicial & Referencia & Óptimo \\
\hline $\mathrm{x}_{1}$ & $(0.0,3.0)$ & 0.0 & 2.718 \\
Presión de salida (MPa) & 14.00 & 14.00 & 11.282 \\
& 13.99 & & \\
$\begin{array}{l}\text { Número de evaluaciones de la } \\
\text { función objetivo }\end{array}$ & & & 30 \\
\hline
\end{tabular}

Tabla 5.9: Valores de la optimización del RIT139 para 1 parámetro.

En la figura 5.11 se presentan los resultados obtenidos para la temperatura de la pared en función de la altura para los casos base y el de la presión de salida óptima comparados con los valores del experimento. En ambos cálculos el punto en el que el RELAP5/Mod3.2 predice que se produce el secado se encuentra a una altura superior a la del experimento. La curva que calcula el caso de la presión óptima incluso desplaza más el punto de secado que la del caso base, sin embargo en la zona anterior al punto de secado la temperatura calculada en este caso coincide con la experimental, mientras que el caso base presenta una discrepancia para cada punto de esta zona. Además, las temperaturas después de producirse el secado son mayores en el caso de la presión de salida optimizada. Por estos dos motivos el error de la evolución completa es menor aunque el punto se encuentre desplazado.

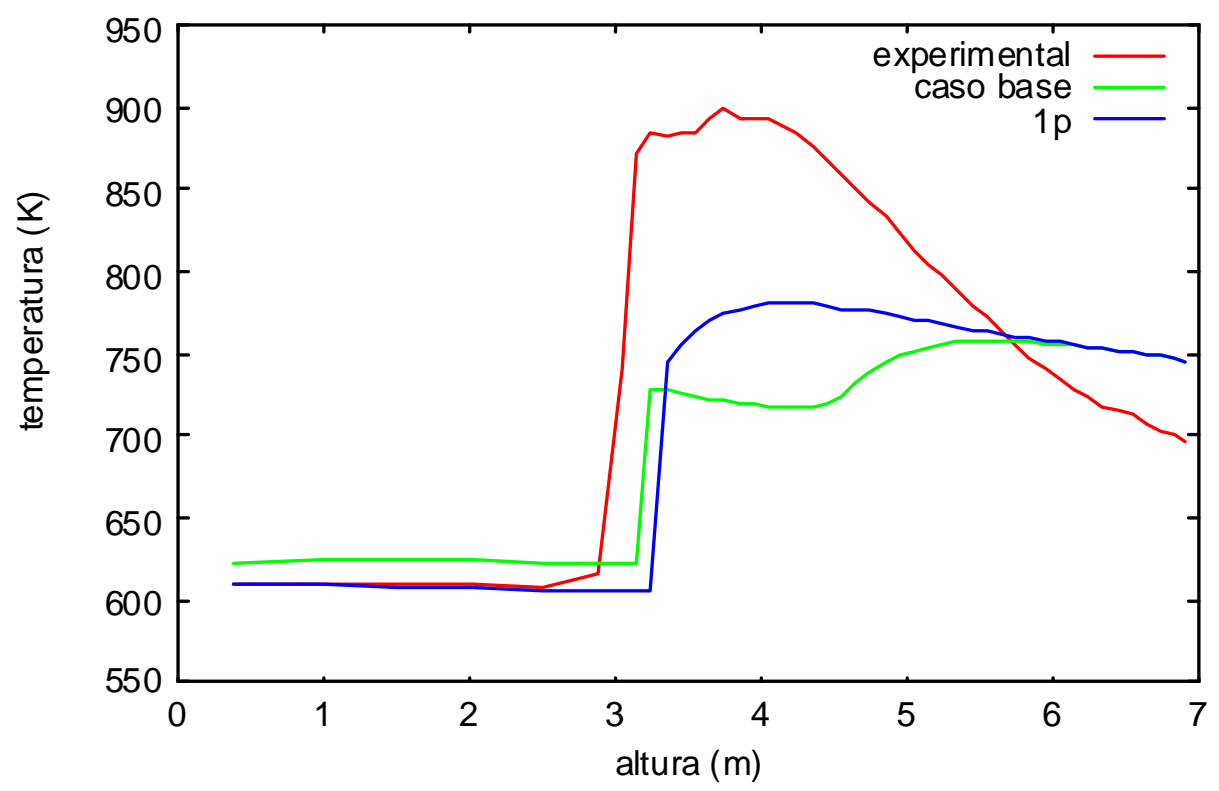

Figura 5.11: Perfil axial de temperatura. Optimización 1 parámetro RIT139.

Siguiendo con el procedimiento del experimento anterior se amplía el problema de optimización con la búsqueda simultánea de las presiones a la entrada y a la salida de la tubería. En este caso se han tenido en cuenta las mismas limitaciones que en el caso 
anterior (RIT136) en cuanto al intervalo en el cual se pueden encontrar las variables de forma que el problema tenga significado físico. Los valores del símplice inicial, el valor óptimo alcanzado de $\mathrm{x}_{1} \mathrm{y}_{2}$, así como las presiones a la entrada y a la salida de la tubería que definen para este problema se presentan en la tabla 5.10.

\begin{tabular}{|c|c|c|c|}
\hline & Inicial & Referencia & Óptimo \\
\hline \multirow{3}{*}{$\left(\mathrm{x}_{1}, \mathrm{x}_{2}\right)$} & $(1.4,0.0)$ & $(1.4,0.0)$ & $(1.4,2.754)$ \\
\hline & $(2.4,0.0)$ & & \\
\hline & $(1.4,1.0)$ & & \\
\hline \multirow[t]{3}{*}{ Presión de entrada (MPa) } & 14.00 & 14.00 & 14.00 \\
\hline & 24.00 & & \\
\hline & 14.00 & & \\
\hline \multirow[t]{3}{*}{ Presión de salida (MPa) } & 14.00 & 14.00 & 11.246 \\
\hline & 21.00 & & \\
\hline & 15.00 & & \\
\hline $\begin{array}{l}\text { Número de evaluaciones de la } \\
\text { función objetivo }\end{array}$ & & & 101 \\
\hline
\end{tabular}

Tabla 5.10: Valores de la optimización del RIT139 para 2 parámetros.

Utilizando estas presiones el perfil axial de la temperatura en la pared calculado por el RELAP5/Mod3.2, es el que se muestra en la figura 5.12. En esta figura se ha mantenido el perfil que se obtiene con la optimización de un sólo parámetro (presión a la salida) para hacer notar que no aparece ninguna mejora del resultado al introducir esta nueva variable de búsqueda. Es decir, el valor de la presión a la entrada de la tubería suministrada en (Nilsson1993) es el mejor valor que puede tomar la variable para conseguir un perfil de temperatura lo más ajustado al experimental en estas condiciones.

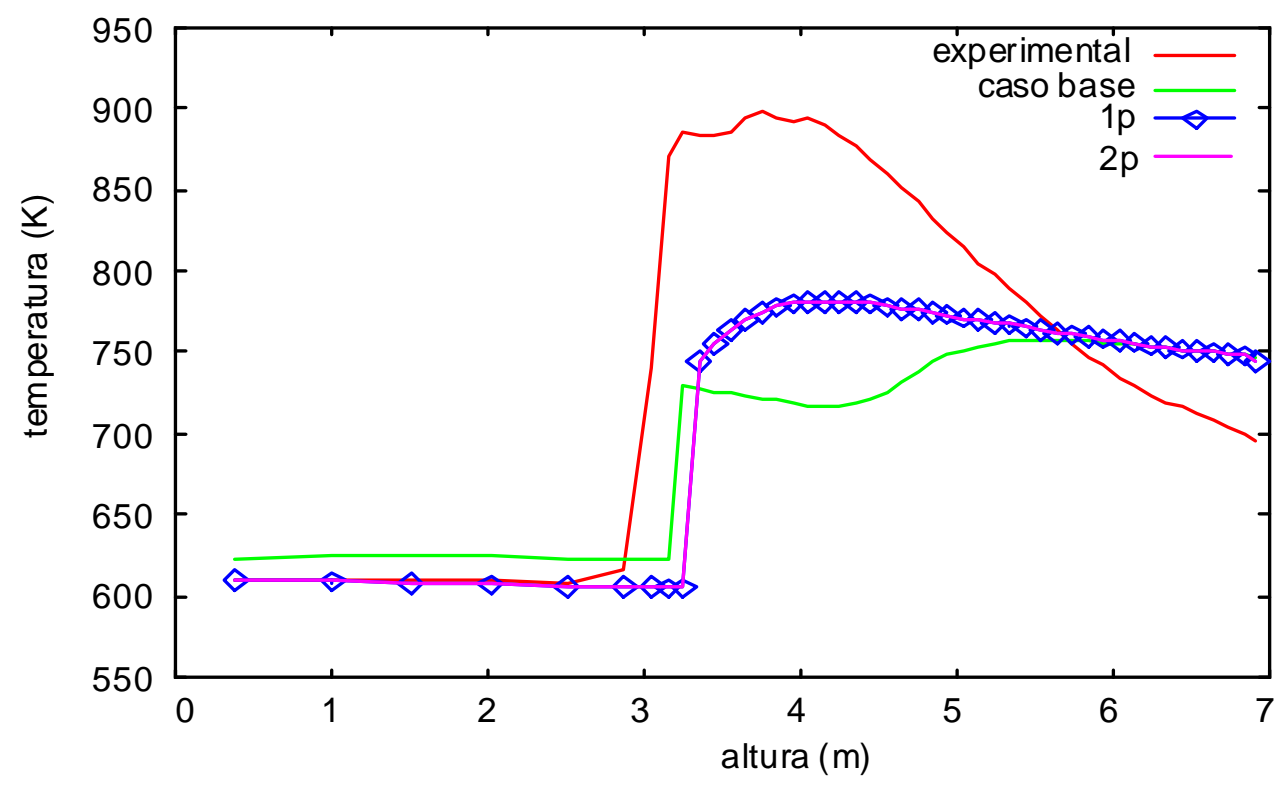

Figura 5.12: Perfil axial de temperatura. Optimización 2 parámetros RIT193. 
Para completar el estudio se ha ampliado la búsqueda a tres parámetros, de manera análoga al caso anterior, introduciendo como variable de búsqueda el caudal de agua de entrada a la tubería. Para esta última optimización, los valores del símplice inicial utilizado, el valor óptimo alcanzado de $\mathrm{x}_{1}, \mathrm{x}_{2} \mathrm{y} \mathrm{x}_{3}$, así como las correspondientes presiones a la entrada y a la salida de la tubería y el caudal de agua, tanto la referencia como los resultados óptimos alcanzados, se presentan en la tabla 5.11.

\begin{tabular}{llll}
\hline & Inicial & Referencia & Óptimo \\
\hline$\left(\mathrm{x}_{1}, \mathrm{x}_{2}, \mathrm{X}_{3}\right)$ & $(1.4,0.0,3.439)$ & $(1.4,0.0,3.439)$ & $(1.274,0.275,2.825)$ \\
& $(0.4,0.0,3.439)$ & & \\
& $(1.4,1.0,3.439)$ & & 14.08 \\
& $(1.4,0.0,4.439)$ & & \\
Presión de entrada (MPa) & 14.00 & 14.00 & 11.67 \\
& 4.00 & & \\
Presión de salida (MPa) & 14.00 & 14.00 & 0.2625 \\
& 14.00 & & \\
& 14.00 & & \\
Caudal de entrada (Kg /s) & 14.00 & 0.3439 & 204 \\
& 15.00 & & \\
& 14.00 & & \\
Número de evaluaciones de la & 0.3439 & & \\
función objetivo & 0.3439 & & \\
\hline
\end{tabular}

Tabla 5.11: Valores de la optimización del RIT139 para 3 parámetros.

La evolución de la temperatura de la pared calculada por el RELAP5/Mod3.2 para los valores optimizados de estos tres parámetros, junto con la experimental y la del caso base, se muestra en la figura 5.13. En esta gráfica se observa que con respecto al caso base se mejora tanto la localización del punto de secado, que se adelanta hasta coincidir con la medida experimental, como el valor de las temperaturas antes y después del mismo, las cuales coinciden con las temperaturas medidas en las zonas anteriores al punto de secado y se acercan más a los datos experimentales en la zona posterior, aunque en esta última zona no llegan a alcanzar los valores de la curva experimental. 


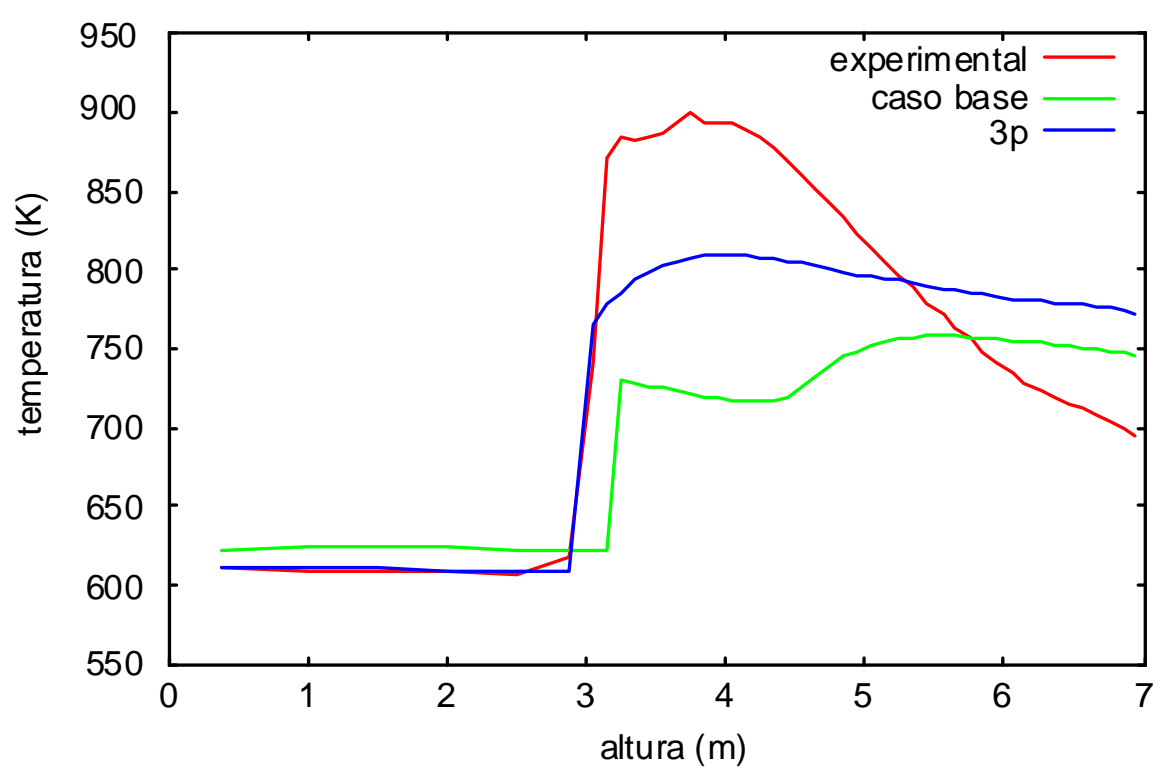

Figura 5.13: Perfil axial de temperatura. Optimización para 3 parámetros RIT139.

$\mathrm{Si}$ se comparan los perfiles de temperatura calculados con los resultados de las optimizaciones anteriores, figuras 5.11, 5.12, y 5.13 se observa que en este caso el RELAP5/Mod3.2 es capaz de reproducir el punto en el que se produce el secado, lo que supone una mejora respecto a los resultados de las anteriores optimizaciones y respecto al caso base. Además, los valores de temperatura que se alcanzan después de producirse el secado son superiores a los calculados con la optimización de dos parámetros. En esta última optimización se encuentra que el valor máximo de temperatura es de $809.45^{\circ} \mathrm{K}$ y en la anterior es de $781.22{ }^{\circ} \mathrm{K}$, sin embargo todavía quedan alejadas del valor experimental que alcanza un máximo de $899.25^{\circ} \mathrm{K}$.

Finalmente, indicar que tampoco en el problema de optimización de tres parámetros se obtiene un cambio de la presión a la entrada de la tubería, igual que ocurría en el caso de considerar dos parámetros en la optimización.

\subsubsection{Consideraciones finales de la optimización}

En los dos experimentos estudiados a los que se ha aplicado la metodología de búsqueda y optimización se han conseguido unas combinaciones de valores de los parámetros que mejoran el cálculo de la distribución de la temperatura de la pared proporcionada por el RELAP5/Mod3.2. Por lo que se puede afirmar que es una metodología general que no depende del caso de aplicación.

En cuanto a la diferencia entre los valores que se proporcionan para el caso base y los nuevos valores encontrados que mejoran el cálculo del RELAP5/mod3.2, esta diferencia puede deberse a que ha habido un error en la medición de los datos experimentales de 
estos parámetros o a que las correlaciones que utiliza el código no proporcionan un cálculo correcto y necesitan condiciones diferentes de funcionamiento.

En cualquiera de las optimizaciones realizadas se aprecia una diferencia entre los cálculos y los datos experimentales en el valor de la temperatura después de producirse el secado, que parece deberse, tal y como se indica en el estudio (Nilsson1993), a los modelos de transferencia de calor implementados en el código, por lo que es aconsejable estudiar qué modelos de transferencia de calor está aplicando el código en la simulación de este transitorio para saber si existe algún parámetro dentro del modelo susceptible de ser mejorado.

Según el manual del RELAP5/Mod3 (INEL1995a; INEL1995c) la selección del modelo más adecuado para el cálculo de la transferencia de calor entre la pared y el fluido se realiza a partir de la curva de ebullición. La forma de esta curva viene dada por la figura 5.14, en la cual se presentan las diferentes regiones para cada uno de los regímenes de transferencia de calor.

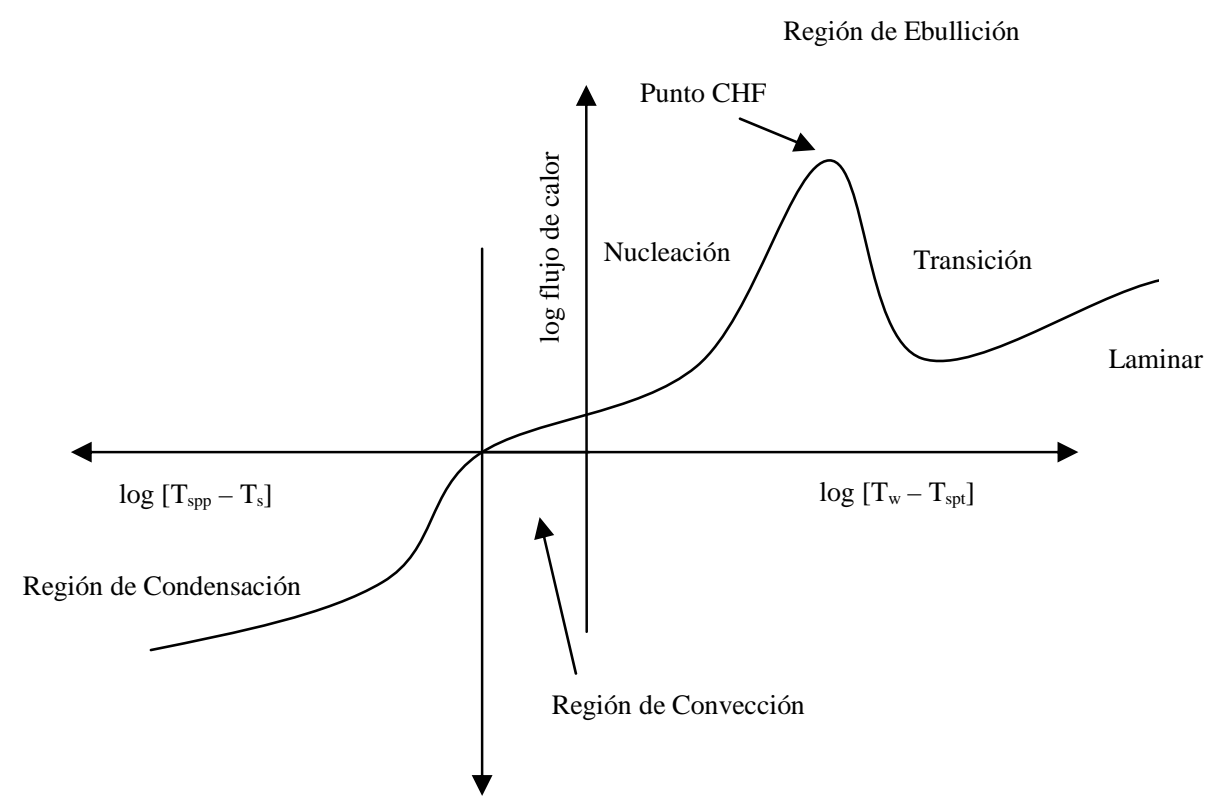

Figura 5.14: Curva de ebullición del RELAP5.

A lo largo del transitorio el código calcula la transferencia de calor en cada una de las estructuras en función de las condiciones que presenta el fluido en cada zona de la tubería. En función del fenómeno de transferencia de calor que se desee simular el código selecciona de forma automática y utiliza las correlaciones más adecuadas. El RELAP5/Mod3.2 indica para cada estructura de calor cuál es el fenómeno que se simula mediante un número que escribe en el fichero de salida. En la tabla 5.12 se muestra el 
número de codificación que asigna el RELAP5/Mod3.2 a cada fenómeno de transferencia de calor que simula y las correlaciones que se utilizan en cada caso.

\begin{tabular}{|c|c|c|}
\hline MODO & Fenómeno de transferencia de calor & Correlaciones \\
\hline 0 & Incondensables-vapor-líquido & $\begin{array}{c}\text { Kays, Dittus-Boelter, ESDU, } \\
\text { Shah } \\
\text { Churchill-Chu, McAdams }\end{array}$ \\
\hline 1 & Fase líquida o líquido supercrítico & $\begin{array}{c}\text { Kays, Dittus-Boelter, ESDU, } \\
\text { Shah } \\
\text { Churchill-Chu, McAdams }\end{array}$ \\
\hline 2 & $\begin{array}{l}\text { Convección a fase líquida en presiones } \\
\text { subcríticas }\end{array}$ & $\begin{array}{c}\text { Kays, Dittus-Boelter, ESDU, } \\
\text { Shah } \\
\text { Churchill-Chu, McAdams }\end{array}$ \\
\hline 3 & Ebullición nucleada subenfriada & Chen \\
\hline 4 & Ebullición nucleada saturada & Chen \\
\hline 5 & $\begin{array}{l}\text { Régimen de ebullición de transición } \\
\text { subenfriada }\end{array}$ & Chen-Sundaram-Ozkaynak \\
\hline 6 & Régimen de ebullición de transición saturada & Chen-Sundaram-Ozkaynak \\
\hline 7 & Ebullición laminar subenfriada & $\begin{array}{c}\text { Bromley, Sun-Gonzales-Tien } \\
\text { Kays, Dittus-Boelter } \\
\text { ESDU, Shah } \\
\text { Churchill-Chu, McAdams }\end{array}$ \\
\hline 8 & Ebullición laminar saturada & $\begin{array}{c}\text { Bromley, Sun-Gonzales-Tien } \\
\text { Kays, Dittus-Boelter } \\
\text { ESDU, Shah } \\
\text { Churchill-Chu, McAdams }\end{array}$ \\
\hline 9 & Bifásico o fase vapor & $\begin{array}{c}\text { Kays, Dittus-Boelter, ESDU, } \\
\text { Shah } \\
\text { Churchill-Chu, McAdams }\end{array}$ \\
\hline 10 & Condensación en película & $\begin{array}{c}\text { Nusselt, Shah, } \\
\text { Colburn-Hougen }\end{array}$ \\
\hline 11 & Condensación en vapor & $\begin{array}{l}\text { Nusselt, Shah, } \\
\text { Colburn-Hougen }\end{array}$ \\
\hline $\begin{array}{l}, 4 \text { para } \\
\text { haces } \\
\text { horizontales }\end{array}$ & Ebullición nucleada & $\begin{array}{c}\text { Forster-Zuber, } \\
\text { Polley-Ralston-Grant } \\
\text { ESDU }\end{array}$ \\
\hline
\end{tabular}

Tabla 5.12: Modos y correlaciones de transferencia de calor.

En las simulaciones realizadas para ambos experimentos no se producen todos estos fenómenos de transferencia de calor, por lo que, con el fin de acotar las condiciones de trabajo e identificar cuáles serán los posibles modelos de transferencia de calor que pueden producir las diferencias entre las temperaturas de la pared medidas en el experimento y las calculadas por el código, se han obtenido los modos de transferencia de calor que se utilizan en la simulación de este transitorio. En las tablas 5.13 y 5.14 se 
muestran los modos correspondientes a los modelos de transferencia de calor para cada una de las estructuras y para cada segundo del transitorio simulados por RELAP5/Mod3.2 en los experimentos RIT136 y RIT139 respectivamente.

\begin{tabular}{|c|c|c|c|c|c|c|c|c|c|c|c|c|c|c|c|c|c|c|c|c|}
\hline \multicolumn{21}{|c|}{ Tiempo (seg) } \\
\hline \multirow{48}{*}{ 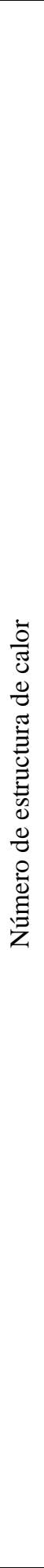 } & & 0 & 1 & 2 & 3 & 4 & 5 & 6 & 7 & 8 & 9 & 10 & 11 & 12 & 13 & 14 & 15 & 16 & 17 & 18 \\
\hline & 1 & 2 & 3 & 3 & 3 & 3 & 3 & 3 & 3 & 3 & 3 & 3 & 3 & 3 & 3 & 3 & 3 & 3 & 3 & 3 \\
\hline & 2 & 2 & 3 & 3 & 3 & 3 & 3 & 3 & 3 & 3 & 3 & 3 & 3 & 3 & 3 & 3 & 3 & 3 & 3 & 3 \\
\hline & 3 & 2 & 3 & 3 & 3 & 3 & 3 & 3 & 3 & 3 & 3 & 3 & 3 & 3 & 3 & 3 & 3 & 3 & 3 & 3 \\
\hline & 4 & 2 & 3 & 3 & 4 & 4 & 4 & 4 & 4 & 4 & 4 & 4 & 4 & 4 & 4 & 4 & 4 & 4 & 4 & 4 \\
\hline & 5 & 2 & 3 & 4 & 4 & 4 & 4 & 4 & 4 & 4 & 4 & 4 & 4 & 4 & 4 & 4 & 4 & 4 & 4 & 4 \\
\hline & 6 & 2 & 3 & 4 & 4 & 4 & 4 & 4 & 4 & 4 & 4 & 4 & 4 & 4 & 4 & 4 & 4 & 4 & 4 & 4 \\
\hline & 7 & 2 & 3 & 4 & 4 & 4 & 4 & 4 & 4 & 4 & 4 & 4 & 4 & 4 & 4 & 4 & 4 & 4 & 4 & 4 \\
\hline & 8 & 2 & 3 & 4 & 4 & 4 & 4 & 4 & 4 & 4 & 4 & 4 & 4 & 4 & 4 & 4 & 4 & 4 & 4 & 4 \\
\hline & 9 & 2 & 3 & 4 & 4 & 4 & 4 & 4 & 4 & 4 & 4 & 4 & 4 & 4 & 4 & 4 & 4 & 4 & 4 & 4 \\
\hline & 10 & 2 & 3 & 4 & 4 & 4 & 4 & 4 & 4 & 4 & 4 & 4 & 4 & 4 & 4 & 4 & 4 & 4 & 4 & 4 \\
\hline & 11 & 2 & 3 & 4 & 4 & 4 & 4 & 4 & 4 & 4 & 4 & 4 & 4 & 4 & 4 & 4 & 4 & 4 & 4 & 4 \\
\hline & 12 & 2 & 3 & 4 & 4 & 4 & 4 & 4 & 4 & 4 & 4 & 4 & 4 & 4 & 4 & 4 & 4 & 4 & 4 & 4 \\
\hline & 13 & 2 & 3 & 4 & 4 & 4 & 4 & 4 & 4 & 4 & 4 & 4 & 4 & 4 & 4 & 4 & 4 & 4 & 4 & 4 \\
\hline & 14 & 2 & 3 & 4 & 4 & 4 & 4 & 4 & 4 & 4 & 4 & 4 & 4 & 4 & 4 & 4 & 4 & 4 & 4 & 4 \\
\hline & 15 & 2 & 3 & 4 & 4 & 4 & 4 & 4 & 4 & 4 & 4 & 4 & 4 & 4 & 4 & 4 & 4 & 4 & 4 & 4 \\
\hline & 16 & 2 & 3 & 4 & 4 & 4 & 4 & 4 & 4 & 4 & 4 & 4 & 4 & 4 & 4 & 4 & 4 & 4 & 4 & 4 \\
\hline & 17 & 2 & 3 & 4 & 4 & 4 & 4 & 4 & 4 & 4 & 4 & 4 & 4 & 4 & 4 & 4 & 4 & 4 & 4 & 4 \\
\hline & 18 & 2 & 3 & 4 & 4 & 4 & 4 & 4 & 4 & 4 & 4 & 4 & 4 & 4 & 4 & 4 & 4 & 4 & 4 & 4 \\
\hline & 19 & 2 & 3 & 4 & 4 & 4 & 4 & 4 & 4 & 4 & 4 & 4 & 4 & 4 & 4 & 4 & 4 & 4 & 4 & 4 \\
\hline & 20 & 2 & 3 & 4 & 4 & 4 & 4 & 4 & 4 & 4 & 4 & 4 & 4 & 4 & 4 & 4 & 4 & 4 & 4 & 4 \\
\hline & 21 & 2 & 3 & 4 & 4 & 4 & 4 & 4 & 4 & 4 & 4 & 4 & 4 & 4 & 4 & 4 & 4 & 4 & 4 & 4 \\
\hline & 22 & 2 & 3 & 4 & 4 & 4 & 4 & 4 & 4 & 4 & 4 & 4 & 4 & 4 & 4 & 4 & 4 & 4 & 4 & 4 \\
\hline & 23 & 2 & 3 & 4 & 4 & 4 & 4 & 4 & 4 & 4 & 4 & 4 & 4 & 4 & 4 & 4 & 4 & 4 & 4 & 4 \\
\hline & 24 & 2 & 3 & 4 & 4 & 4 & 4 & 4 & 4 & 4 & 4 & 4 & 4 & 4 & 4 & 4 & 4 & 4 & 4 & 4 \\
\hline & 25 & 2 & 3 & 4 & 4 & 4 & 4 & 4 & 4 & 4 & 4 & 4 & 4 & 4 & 4 & 4 & 4 & 4 & 4 & 4 \\
\hline & 26 & 2 & 3 & 4 & 4 & 4 & 4 & 4 & 4 & 4 & 4 & 4 & 4 & 4 & 4 & 4 & 4 & 4 & 4 & 4 \\
\hline & 27 & 2 & 3 & 4 & 4 & 4 & 4 & 4 & 4 & 4 & 4 & 4 & 4 & 4 & 4 & 4 & 4 & 4 & 4 & 4 \\
\hline & 28 & 2 & 3 & 4 & 4 & 4 & 4 & 4 & 4 & 4 & 4 & 4 & 4 & 4 & 4 & 4 & 4 & 4 & 4 & 4 \\
\hline & 29 & 2 & 3 & 4 & 4 & 4 & 4 & 4 & 4 & 4 & 4 & 4 & 4 & 4 & 4 & 4 & 4 & 4 & 4 & 4 \\
\hline & 30 & 2 & 3 & 4 & 4 & 4 & 4 & 4 & 4 & 4 & 4 & 4 & 4 & 4 & 4 & 4 & 4 & 4 & 4 & 4 \\
\hline & 31 & 2 & 3 & 4 & 4 & 4 & 4 & 4 & 4 & 4 & 4 & 4 & 4 & 4 & 4 & 4 & 4 & 4 & 4 & 4 \\
\hline & 32 & 2 & 3 & 4 & 4 & 4 & 4 & 4 & 4 & 6 & 6 & 6 & 8 & 8 & 8 & 8 & 8 & 8 & 8 & 8 \\
\hline & 33 & 2 & 3 & 4 & 4 & 4 & 4 & 4 & 4 & 6 & 6 & 8 & 8 & 8 & 8 & 8 & 8 & 8 & 8 & 8 \\
\hline & 34 & 2 & 3 & 4 & 4 & 4 & 4 & 4 & 6 & 6 & 6 & 8 & 8 & 8 & 8 & 8 & 8 & 8 & 8 & 8 \\
\hline & 35 & 2 & 4 & 4 & 4 & 4 & 4 & 4 & 6 & 6 & 8 & 8 & 8 & 8 & 8 & 8 & 8 & 8 & 8 & 8 \\
\hline & 36 & 2 & 4 & 4 & 4 & 4 & 4 & 4 & 6 & 6 & 8 & 8 & 8 & 8 & 8 & 8 & 8 & 8 & 8 & 8 \\
\hline & 37 & 2 & 4 & 4 & 4 & 4 & 4 & 4 & 6 & 6 & 8 & 8 & 8 & 8 & 8 & 8 & 8 & 8 & 8 & 8 \\
\hline & 38 & 2 & 4 & 4 & 4 & 4 & 4 & 6 & 6 & 6 & 8 & 8 & 8 & 8 & 8 & 8 & 8 & 8 & 8 & 8 \\
\hline & 39 & 2 & 4 & 4 & 4 & 4 & 4 & 6 & 6 & 6 & 8 & 8 & 8 & 8 & 8 & 8 & 8 & 8 & 8 & 8 \\
\hline & 40 & 2 & 4 & 4 & 4 & 4 & 4 & 6 & 6 & 6 & 8 & 8 & 8 & 8 & 8 & 8 & 8 & 8 & 8 & 8 \\
\hline & 41 & 2 & 4 & 4 & 4 & 4 & 4 & 6 & 6 & 8 & 8 & 8 & 8 & 8 & 8 & 8 & 8 & 8 & 8 & 8 \\
\hline & 42 & 2 & 4 & 4 & 4 & 4 & 4 & 6 & 6 & 8 & 8 & 8 & 8 & 8 & 8 & 8 & 8 & 8 & 8 & 8 \\
\hline & 43 & 2 & 4 & 4 & 4 & 4 & 4 & 6 & 6 & 8 & 8 & 8 & 8 & 8 & 8 & 8 & 8 & 8 & 8 & 8 \\
\hline & 44 & 2 & 4 & 4 & 4 & 4 & 4 & 6 & 6 & 8 & 8 & 8 & 8 & 8 & 8 & 8 & 8 & 8 & 8 & 8 \\
\hline & 45 & 2 & 4 & 4 & 4 & 4 & 4 & 6 & 6 & 8 & 8 & 8 & 8 & 8 & 8 & 8 & 8 & 8 & 8 & 8 \\
\hline & 46 & 2 & 4 & 4 & 4 & 4 & 4 & 6 & 6 & 8 & 8 & 8 & 8 & 8 & 8 & 8 & 8 & 8 & 8 & 8 \\
\hline & 47 & 2 & 4 & 4 & 4 & 4 & 4 & 6 & 6 & 8 & 8 & 8 & 8 & 8 & 8 & 8 & 8 & 8 & 8 & 8 \\
\hline
\end{tabular}

Tabla 5.13: Modos de transferencia de calor en el RIT136. 
Tiempo (seg)

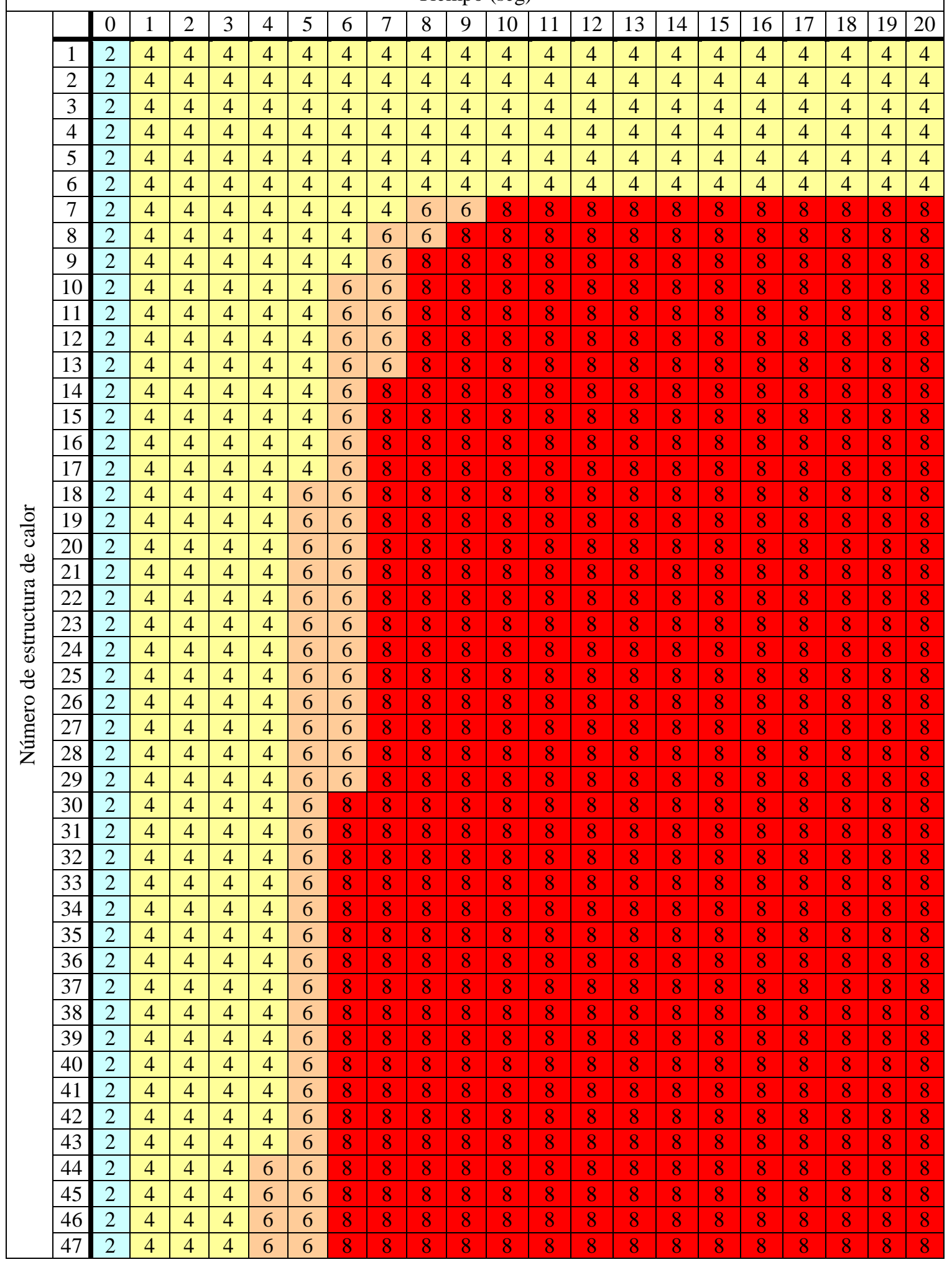

Tabla 5.14: Modos de transferencia de calor en el RIT139. 
En la tabla 5.13 y 5.14 se muestran, por medio de su codificación, los fenómenos de transferencia de calor que simula el código RELAP5/Mod3.2 en cada una de las estructuras de calor y en cada segundo de transitorio para el experimento RIT136 y el RIT139 respectivamente.

Sabiendo, de las correlaciones implementadas en el código, expuestas en la tabla 5.12, las que se utilizan en cada modo de transferencia de calor, el siguiente paso para mejorar el cálculo realizado por el código consiste en identificar las correlaciones de los modelos que difieran significativamente del comportamiento experimental y su posterior mejora. En principio, teniendo en cuenta que las diferencias entre el valor de la temperatura calculado y el experimental difieren después de producirse el secado, las correlaciones candidatas deberían ser las que se utilizan en esta zona. En ambos casos, atendiendo únicamente al último segundo del transitorio que es en el que se ha medido la temperatura en la instalación, el punto de secado se produce a una altura de $2.875 \mathrm{~m}$ en el caso RIT136 y 5.55 m en el RIT139, que coincide con el cambio de modo de transferencia de calor de 4 a 8. Por tanto, debido a que las diferencias observadas en el perfil de la temperatura de la pared se producen después del secado, las correlaciones candidatas a ser estudiadas son las que se utilizan en el modo de transferencia de calor 8 que corresponde a la ebullición laminar saturada y cuyas correlaciones se muestran en la tabla 5.12.

Este estudio, sin embargo, queda fuera del alcance de la presente tesis puesto que los parámetros susceptibles de mejora en cada una de las correlaciones no se encuentran accesibles desde el fichero de entrada del RELAP5, lo cual es una condición indispensable para que este método de búsqueda sea eficiente.

Además, se debe contar con el hecho que el secado no se produce de forma inmediata en todas las estructuras de calor; así por ejemplo si consideramos que el modo de transferencia de calor 8 es el que introduce el error en el cálculo, en la tabla 5.13 que muestra los modos de transferencia en el experimento, se observa que a los 8 segundos de transitorio el código ya empieza a utilizar este modelo en el cálculo de la temperatura de las estructuras superiores, sin embargo el error no se calcula hasta los 18 segundos. Por tanto, durante todo este tiempo el error se va acumulando. Este efecto podría mitigarse si se dispusiese de datos para varios instantes de tiempo en cada una de las estructuras de calor, con los que se confeccionaría una matriz de error con la que se debería realizar de nuevo la optimización puesto que podría llevarnos a unos valores de los parámetros que ajustarán más el cálculo a la curva experimental. 
Capítulo 5 


\section{Capítulo 6}

\section{Aplicación a modelos reducidos de reactor}

\subsection{Introducción}

El uso de los modelos reducidos para el estudio de reactores nucleares está bastante extendido, ya que permite entender de forma cualitativa los fenómenos físicos que rigen su comportamiento (Su2001). Este tipo de análisis posibilita la realización de estudios de sensibilidad sobre un determinado proceso que tiene lugar en el reactor y la identificación de los parámetros que lo afectan, de una manera rápida y sencilla. No obstante, hay que tener en cuenta que para la simulación de transitorios complicados, como, por ejemplo, los accidentes de pérdida de refrigerante, es necesario realizar el análisis del transitorio mediante modelos detallados implementados en códigos de simulación como el RELAP o el TRAC.

Entre las aplicaciones de los modelos reducidos de reactores se pueden encontrar los diseños de controles para mantener la potencia del reactor estable ante distintas condiciones de carga de la turbina (Park1992; Park1993; Accorsi1999). Otra aplicación de los modelos reducidos de reactores PWR ha sido la monitorización del coeficiente asociado a la temperatura del moderador en la expresión de la reactividad a partir de medidas de ruido (Thomas1991) ya que los organismos reguladores requieren la determinación de este parámetro al principio y al final de cada ciclo de combustible en la mayoría de plantas PWR. 
Los modelos reducidos de reactores BWR han sido utilizados con notable éxito para avanzar en la comprensión de las inestabilidades que tienen lugar en este tipo de plantas (March-Leuba1986; Karve1997).

Los modelos reducidos de reactor se basan principalmente en las ecuaciones de la cinética puntual para describir el balance neutrónico en el interior del núcleo del reactor $\mathrm{y}$ en modelos de equilibrio térmico para dar cuenta del intercambio energético en los distintos lazos de un reactor del tipo PWR. En el caso de los reactores BWR se utilizan simplificaciones de las ecuaciones de la masa, energía y momento para las distintas fases del refrigerante que describen los procesos de intercambio y transmisión de calor.

En todos estos modelos reducidos aparecen una serie de parámetros efectivos que varían dependiendo de la geometría de la planta y de sus condiciones de funcionamiento. Por ello, para ajustar el modelo a una planta en concreto es necesario estimar estos parámetros. Para obtener los valores de los mismos se propone la utilización de la metodología de búsqueda y optimización de parámetros basada en el algoritmo de búsqueda multidireccional (MDS), de forma análoga a cómo se ha hecho en los capítulos anteriores.

En este capítulo se presenta un modelo reducido de una planta PWR cuyos parámetros se ajustarán a una planta de referencia a partir de los datos proporcionados por el código de simulación RELAP5/Mod3.2 utilizando una metodología de optimización de parámetros basada en el método MDS y se discutirán algunos de los aspectos relacionados con el modelo ajustado.

\subsection{Planta de referencia}

Como ya se ha comentado, se pretende construir un modelo reducido que describa el comportamiento de una planta PWR típica. En particular, el modelo detallado de instalación utilizado como referencia es el que se suministra con el paquete de instalación del RELAP5, que constituye uno de los casos de verificación de este código. En concreto, corresponde al fichero "typpwr.i" en el cual está modelizada una planta PWR de cuatro lazos y $3600 \mathrm{Mw}$ de potencia sobre la que se produce una rotura pequeña en uno de sus lazos.

En el modelo de RELAP se han unificado los tres lazos intactos en un solo lazo por el que circula una cantidad de agua igual a la suma de los tres lazos individuales, mientras que el lazo en el que se produce la rotura se ha modelizado aparte, constituyendo pues un modelo de dos lazos asimétrico. 
En total la nodalización del fichero de entrada está formada por 139 volúmenes de control y 142 juntas que definen la geometría completa de la instalación, y 83 estructuras de calor para simular los intercambios energéticos que se producen en la planta. En esta nodalización se encuentran modelizados los componentes básicos de una planta PWR como son los generadores de vapor, presionador, acumulador y la vasija del reactor.

Así, en cada uno de los dos lazos considerados en la nodalización se ha modelizado un generador de vapor. Los tubos en U del primario de los generadores de vapor están modelizados mediante un componente PIPE (Inel1995b), mientras que el secundario está compuesto por varios volúmenes. El acoplamiento entre el primario y el secundario de los generadores de vapor se realiza mediante una estructura de calor que simula el intercambio de energía entre ambos.

Además, la nodalización dispone en cada lazo de un acumulador representado por un componente ACCUM implementado en el RELAP, que simula de forma específica las características y el comportamiento de los acumuladores. Otro de los componentes especiales simulados son la bombas del primario, situadas también cada una en un lazo, y que se han representado mediante el componente PUMP de RELAP, específico para la simulación del comportamiento de las bombas.

Otro componente importante dentro de una planta PWR es el presionador, cuya nodalización se ha hecho mediante un componente PIPE vertical de seis volúmenes que conecta a través de las válvulas de alivio, representadas por componentes VALVE, con la contención que se ha supuesto como un componente TMDPVOL que presenta las características de presión y temperatura de la atmósfera. La parte inferior del presionador se encuentra unida al lazo intacto del primario.

Por último, la vasija del reactor está compuesta por 17 volúmenes y 15 estructuras de calor que modelizan tanto su geometría como los intercambios energéticos entre cada uno de los componentes de la vasija y el refrigerante-moderador. De todos los componentes que la forman cabe citar el núcleo que está representado por un componente PIPE vertical de 6 volúmenes, sobre los que se cede el calor generado por el combustible, proceso simulado por una estructura de calor.

La generación de potencia neutrónica se describe mediante un modelo de cinética puntual con seis grupos de precursores cuyos parámetros corresponden a la opción ANS79-1. Para la expresión de la reactividad se utiliza un modelo local con un coeficiente asociado a la temperatura del moderador para cada uno de los volúmenes del núcleo y otro asociado a la temperatura del combustible en cada división axial de la extructura de calor que simula las varillas de combustible (Inel1995a;Inel1995b). 
Puesto que este fichero de entrada está inicialmente preparado para la simulación de un accidente de pérdida de refrigerante debido a una rotura pequeña en uno de sus lazos en el que deben actuar distintas salvaguardias, el modelo de la planta contiene un modelo simple de estas salvaguardias y una lógica de actuación controlada mediante TRIPS (Inel1995b).

En nuestro caso, debido a que en los accidentes de pérdida de refrigerante se producen fenómenos físicos complejos que no va a ser posible simular mediante el modelo reducido, se ha optado por la simulación de un transitorio más simple que es típico en la operación normal de la planta. Este transitorio consiste en simular el movimiento de las barras de control, lo cual se traduce en una inserción de reactividad. Para simular este nuevo transitorio se ha partido del modelo inicial de la planta desactivando completamente la lógica de actuación de las salvaguardias y controlando la inserción de reactividad mediante una tabla accionada por un nuevo TRIP, manteniendo en todo momento la geometría inicial del modelo. Este nuevo fichero de entrada se presenta en el Apéndice 3 de la presente memoria.

\subsection{Modelo reducido de reactor PWR}

Como ya se ha comentado, el objeto de este capítulo es construir un modelo reducido de reactor PWR con dos lazos, sobre el que se va a aplicar la metodología de búsqueda y optimización de parámetros. Para el diseño de este modelo tomaremos como base el esquema presentado en la figura 6.1.

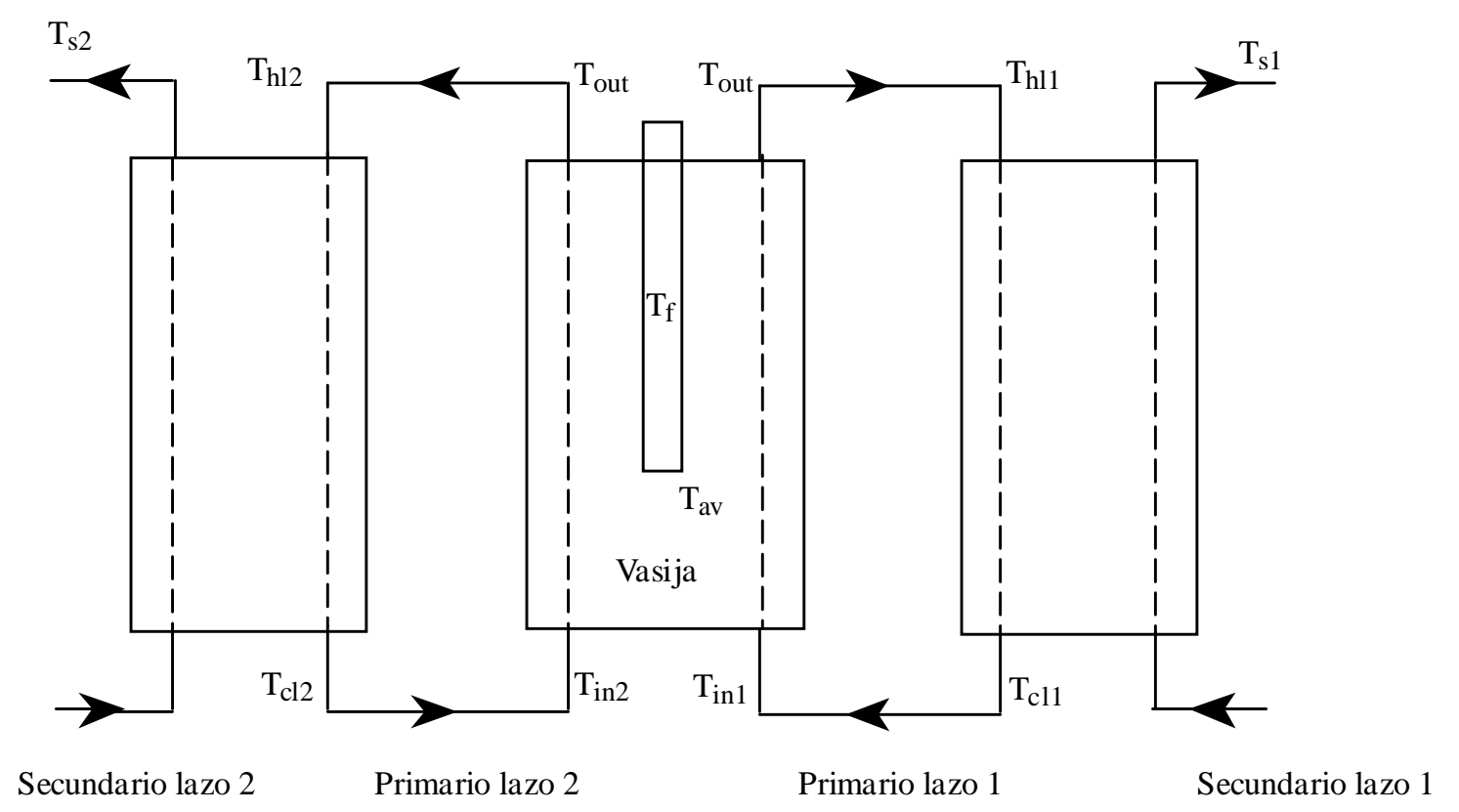

Figura 6.1: Esquema del modelo reducido de reactor PWR de dos lazos. 
En el desarrollo de un modelo simplificado de reactor se deben tener en cuenta tanto la parte termohidráulica como la neutrónica del problema. En primer lugar obtendremos las ecuaciones de la cinética puntual, que constituyen un conjunto de ecuaciones simplificadas para dar cuenta de la evolución de la potencia neutrónica en el núcleo del reactor.

Bajo condiciones generales el balance neutrónico en el interior del núcleo se puede describir aproximadamente mediante la ecuación de la difusión neutrónica en un grupo de energía y K grupos de precursores de neutrones (Henry1982), que es de la forma

$$
\begin{aligned}
& \frac{1}{\mathrm{v}} \frac{\partial \Phi}{\partial \mathrm{t}}=\nabla(\mathrm{D} \nabla \Phi)+(1-\beta) \nu \Sigma_{\mathrm{f}} \Phi-\Sigma_{\mathrm{a}} \Phi+\sum_{\mathrm{i}=1}^{\mathrm{K}} \lambda_{\mathrm{i}} \mathrm{C}_{\mathrm{i}} \\
& \frac{\partial \mathrm{C}_{\mathrm{i}}}{\partial \mathrm{t}}=\beta_{\mathrm{i}} v \Sigma_{\mathrm{f}} \Phi-\lambda_{\mathrm{i}} \mathrm{C}_{\mathrm{i}}, \quad \mathrm{i}=1, \ldots, \mathrm{K}
\end{aligned}
$$

donde $\Phi$ es el flujo neutrónico, y $\mathrm{C}_{\mathrm{i}}$ es la concentración del grupo i-ésimo de precursores de neutrones. Más detalles sobre el significado de las distintas constantes de la ecuación $\mathrm{v}, \beta, \mathrm{D}, \Sigma_{\mathrm{a}}, \lambda_{\mathrm{i}}, v \Sigma_{\mathrm{f}}, \beta_{\mathrm{i}}$, pueden encontrarse en (Henry1982).

Introducimos el operador de pérdidas neutrónicas

$$
\mathrm{L}=-\bar{\nabla}(\mathrm{D} \bar{\nabla})+\Sigma_{\mathrm{a}}
$$

y el operador de producción neutrónica

$\mathrm{M}=\mathrm{v} \Sigma_{\mathrm{f}}$

Además, reescribimos los operadores de pérdidas y producción de la siguiente manera

$$
\mathrm{L}=\mathrm{L}^{0}+\delta \mathrm{L} ; \quad \mathrm{M}=\mathrm{M}^{0}+\delta \mathrm{M}
$$

donde $\mathrm{L}^{0}$ y $\mathrm{M}^{0}$ son los operadores de pérdidas y de producción en estado estacionario, respectivamente.

Así, las ecuaciones (6.1) y (6.2) se pueden escribir como

$$
\frac{1}{\mathrm{v}} \frac{\partial \Phi}{\partial \mathrm{t}}+\left(\mathrm{L}^{0}+\delta \mathrm{L}\right) \Phi=(1-\beta)\left(\mathrm{M}^{0}+\delta \mathrm{M}\right) \Phi+\sum_{\mathrm{i}=1}^{\mathrm{K}} \lambda_{\mathrm{i}} \mathrm{C}_{\mathrm{i}}
$$




$$
\frac{\partial \mathrm{C}_{\mathrm{i}}}{\partial \mathrm{t}}=\beta_{\mathrm{i}}\left(\mathrm{M}^{0}+\delta \mathrm{M}\right) \Phi-\lambda_{\mathrm{i}} \mathrm{C}_{\mathrm{i}}, \quad \mathrm{i}=1, \ldots, \mathrm{K}
$$

Suponemos que es posible factorizar el flujo neutrónico según la dependencia espacial y temporal de la forma

$\Phi(\mathrm{x}, \mathrm{t})=\mathrm{N}(\mathrm{t}) \Psi(\mathrm{x})$

donde $\Psi(\mathrm{x})$ es el modo fundamental de la ecuación de los modos lambda (Henry1982)

$\mathrm{L}^{0} \Psi=\frac{1}{\mathrm{k}_{\mathrm{eff}}} \mathrm{M}^{0} \Psi$

De este modo, las ecuaciones (6.3) y (6.4) se expresan como

$$
\begin{aligned}
& \frac{1}{\mathrm{v}} \frac{\mathrm{dN}}{\mathrm{dt}} \Psi+\mathrm{N} \frac{1}{\mathrm{k}_{\mathrm{eff}}} \mathrm{M}^{0} \Psi+\mathrm{N} \delta L \Psi-(1-\beta)\left(\mathrm{M}^{0} \mathrm{~N} \Psi+\delta \mathrm{MN} \Psi\right)-\sum_{\mathrm{i}=1}^{\mathrm{K}} \lambda_{\mathrm{i}} \mathrm{C}_{\mathrm{i}} \\
& \frac{\partial \mathrm{C}_{\mathrm{i}}}{\partial \mathrm{t}}=\beta_{\mathrm{i}} \mathrm{M}^{0} \mathrm{~N} \Psi+\beta_{\mathrm{i}} \delta \mathrm{MN} \Psi-\lambda_{\mathrm{i}} \mathrm{C}_{\mathrm{i}} \quad, \quad \mathrm{i}=1, \ldots, \mathrm{K}
\end{aligned}
$$

introduciendo el producto escalar

$$
\langle\varphi, \eta\rangle=\int_{\mathrm{v}} \varphi(\mathbf{x}) \eta(\mathbf{x}) \mathrm{d} \mathbf{x}
$$

donde la integración se extiende a todo el volumen del núcleo del reactor, se pueden escribir las ecuaciones (6.5) y (6.6) como

$$
\begin{aligned}
& \frac{\mathrm{dN}}{\mathrm{dt}}=\mathrm{N}\left(\left(1-\frac{1}{\mathrm{k}_{\mathrm{eff}}}\right)-\beta\right) \frac{\left\langle\Psi, \mathrm{M}^{0} \Psi\right\rangle}{\left\langle\Psi, \frac{1}{\mathrm{v}} \Psi\right\rangle}+\frac{\langle\Psi,((1-\beta) \delta \mathrm{M}-\delta \mathrm{L}) \Psi\rangle}{\left\langle\Psi, \frac{1}{\mathrm{v}} \Psi\right\rangle} \mathrm{N}+\sum_{\mathrm{i}=1}^{\mathrm{K}} \lambda_{\mathrm{i}} \frac{\left\langle\Psi, \mathrm{C}_{\mathrm{i}}\right\rangle}{\left\langle\Psi, \frac{1}{\mathrm{v}} \Psi\right\rangle}, \\
& \frac{\mathrm{d}\left\langle\Psi, \mathrm{C}_{\mathrm{i}}\right\rangle}{\mathrm{dt}}=\mathrm{N} \beta_{\mathrm{i}}\left\langle\Psi, \mathrm{M}^{0} \Psi\right\rangle+\beta_{\mathrm{i}} \mathrm{N}\langle\Psi, \delta \mathrm{M} \Psi\rangle-\lambda_{\mathrm{i}}\left\langle\Psi, \mathrm{C}_{\mathrm{i}}\right\rangle, \quad \mathrm{i}=1, \ldots, \mathrm{K} .
\end{aligned}
$$


Definiendo el tiempo de generación de neutrones

$\Lambda=\frac{\left\langle\Psi, \frac{1}{\mathrm{~V}} \Psi\right\rangle}{\left\langle\Psi, \mathrm{M}^{0} \Psi\right\rangle}$,

y las amplitudes

$\mathrm{c}_{\mathrm{i}}=\frac{\left\langle\Psi, \mathrm{C}_{\mathrm{i}}\right\rangle}{\left\langle\Psi, \frac{1}{\mathrm{~V}} \Psi\right\rangle}=\frac{\left\langle\Psi, \mathrm{C}_{\mathrm{i}}\right\rangle}{\Lambda}\left\langle\Psi, \mathrm{M}^{0} \Psi\right\rangle \quad, \quad \mathrm{i}=1, \ldots, \mathrm{K}$

al sustituir las ecuaciones quedan

$$
\begin{aligned}
& \frac{\mathrm{dN}}{\mathrm{dt}}=\frac{\mathrm{N}}{\Lambda}\left(\left(1-\frac{1}{\mathrm{k}_{\text {eff }}}\right)+\frac{1}{\left\langle\Psi, \mathrm{M}^{0} \Psi\right\rangle}\langle\Psi,((1-\beta) \delta \mathrm{M}-\delta \mathrm{L}) \Psi\rangle-\beta\right)+\sum_{\mathrm{i}=1}^{\mathrm{K}} \lambda_{\mathrm{i}} \mathrm{c}_{\mathrm{i}} \\
& \frac{\mathrm{dc}_{\mathrm{i}}}{\mathrm{dt}}=\beta_{\mathrm{i}} \frac{\mathrm{N}}{\Lambda}+\beta_{\mathrm{i}} \frac{\mathrm{N}}{\Lambda} \frac{1}{\left\langle\Psi, \mathrm{M}^{0} \Psi\right\rangle}\langle\Psi, \delta \mathrm{M} \Psi\rangle-\lambda_{\mathrm{i}} \mathrm{c}_{\mathrm{i}}, \mathrm{i}=1, \ldots, \mathrm{K} .
\end{aligned}
$$

Se define la reactividad, medida en dólares, de la siguiente manera

$$
\rho=\frac{1}{\beta}\left(1-\frac{1}{\mathrm{k}_{\mathrm{eff}}}+\frac{1}{\left\langle\Psi, \mathrm{M}^{0} \Psi\right\rangle}\langle\Psi,((1-\beta) \delta \mathrm{M}-\delta \mathrm{L}) \Psi\rangle\right)
$$

y aproximando

$$
1+\frac{1}{\left\langle\Psi, \mathrm{M}^{0} \Psi\right\rangle}\langle\Psi, \delta \mathrm{M} \Psi\rangle \cong 1
$$

obtenemos las ecuaciones de la cinética puntual

$$
\frac{\mathrm{dN}}{\mathrm{dt}}=\beta \frac{\mathrm{N}}{\Lambda}(\rho-1)+\sum_{\mathrm{i}=1}^{\mathrm{K}} \lambda_{\mathrm{i}} \mathrm{c}_{\mathrm{i}}
$$


$\frac{d c_{i}}{d t}=\beta_{i} \frac{N}{\Lambda}-\lambda c_{i}, i=1, \ldots, K$

Se supone que la potencia generada por el reactor es proporcional a $\mathrm{N}$

$\mathrm{P}=\gamma \mathrm{N}$,

Con lo que las ecuaciones de la cinética puntual quedan de la forma

$$
\begin{aligned}
& \frac{\mathrm{dP}}{\mathrm{dt}}=\beta \frac{\mathrm{P}}{\Lambda}(\rho-1)+\sum_{\mathrm{i}=1}^{\mathrm{K}} \lambda_{\mathrm{i}} \widetilde{\mathrm{c}}_{\mathrm{i}}, \\
& \frac{\mathrm{d} \widetilde{\mathrm{c}}_{\mathrm{i}}}{\mathrm{dt}}=\beta_{\mathrm{i}} \frac{\mathrm{P}}{\Lambda}-\lambda_{\mathrm{i}} \widetilde{\mathrm{c}}_{\mathrm{i}} \quad, \mathrm{i}=1, \ldots, \mathrm{K} .
\end{aligned}
$$

donde $\widetilde{c}_{i}=\gamma c_{i}$.

Para obtener un modelo simplificado que dé cuenta de la transferencia de calor entre el combustible y el refrigerante se realiza un balance energético a partir de las temperaturas medias. Para determinar estas ecuaciones se parte de la ecuación de Fourier, que nos da la distribución de temperatura en las varillas del combustible

$$
\rho_{\mathrm{f}} \mathrm{c}_{\mathrm{pf}} \frac{\partial \mathrm{T}}{\partial \mathrm{t}}=\mathrm{P}+\nabla(\mathrm{k} \nabla \mathrm{T})
$$

siendo $\rho_{\mathrm{f}}$ la densidad del combustible, $\mathrm{c}_{\mathrm{pf}}$ el calor específico a presión constante del combustible, $\mathrm{P}$ la distribución de potencia generada por el combustible y k el coeficiente de transferencia de calor.

Integrando la ecuación (6.11) para toda la región del combustible, V, se tiene

$$
\left\langle\rho_{\mathrm{f}} \mathrm{c}_{\mathrm{pf}}\right\rangle \frac{\partial}{\partial \mathrm{t}} \frac{1}{\mathrm{~V}} \int_{\mathrm{V}} \mathrm{T}(\mathrm{r}) \mathrm{dr}=\langle\mathrm{P}\rangle+\frac{1}{\mathrm{~V}} \int_{\mathrm{V}} \nabla(\mathrm{k} \nabla \mathrm{T}) \mathrm{dr}
$$

donde $\left\langle\rho_{\mathrm{f}} \mathrm{c}_{\mathrm{pf}}\right\rangle$ es un coeficiente efectivo promedio del combustible (March-Leuba1986) y $\langle\mathbf{P}\rangle$ es la potencia media cedida por el combustible. Se introduce la temperatura media del combustible $\mathrm{T}_{\mathrm{f}}$

$$
\mathrm{T}_{\mathrm{f}}=\frac{1}{\mathrm{~V}} \int_{\mathrm{V}} \mathrm{T}(\mathrm{r}) \mathrm{dr}
$$


y utilizando el teorema de Gauss se puede escribir la ecuación (6.12) como

$$
\frac{\mathrm{dT}_{\mathrm{f}}}{\mathrm{dt}}=\frac{\langle\mathrm{P}\rangle}{\left\langle\rho_{\mathrm{f}} \mathrm{c}_{\mathrm{pf}}\right\rangle}+\frac{1}{\mathrm{~V}\left\langle\rho_{\mathrm{f}} \mathrm{c}_{\mathrm{pf}}\right\rangle} \int_{\mathrm{S}} \mathrm{k} \nabla \mathrm{Tds}
$$

donde $\mathrm{S}$ es la superficie de la región del combustible.

Para este modelo se considera una aproximación de la integral de superficie de la siguiente manera (March-Leuba1986)

$\int_{\mathrm{S}} \mathrm{k} \nabla \mathrm{Tds} \approx-\mathrm{UA}\left(\mathrm{T}_{\mathrm{f}}-\mathrm{T}_{\mathrm{av}}\right)$

donde $\mathrm{U}$ es un coeficiente de transferencia de calor efectivo para el núcleo del reactor, $\mathrm{A}$ es un área efectiva de la región del combustible y $\mathrm{T}_{\mathrm{av}}$ es la temperatura media del refrigerante-moderador (véase figura 6.2).

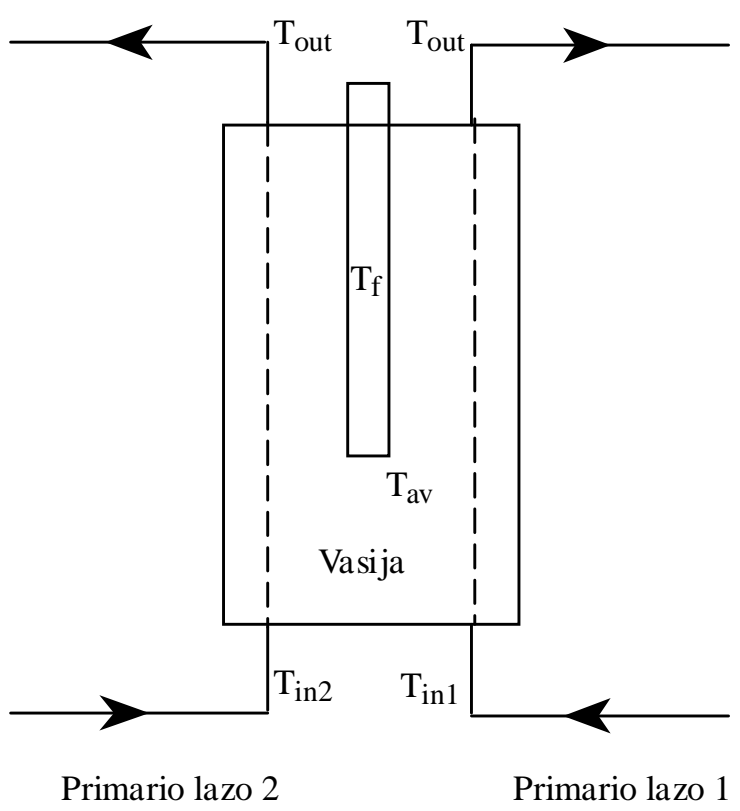

Figura 6.2: Esquema simplificado de la vasija y combustible.

La ecuación (6.13) la rescribimos como (Park1992)

$$
\frac{\partial \mathrm{T}_{\mathrm{f}}}{\mathrm{dt}}=-\left(\frac{\mathrm{UA}}{\mathrm{M}_{\mathrm{f}} \mathrm{c}_{\mathrm{pf}}}\right)\left(\mathrm{T}_{\mathrm{f}}-\mathrm{T}_{\mathrm{av}}\right)+\left(\frac{\mathrm{F}}{\mathrm{M}_{\mathrm{f}} \mathrm{c}_{\mathrm{pf}}}\right) \mathrm{P},
$$


donde $\mathrm{M}_{\mathrm{f}}$ es una masa efectiva del combustible, $\mathrm{F}$ es una constante, y hemos escrito $\mathrm{P}$ para denotar la potencia media.

Mediante un razonamiento similar se obtiene una ecuación para la variación de la temperatura media del moderador en el interior de la vasija del reactor, de la forma

$$
\frac{\partial \mathrm{T}_{\mathrm{av}}}{\mathrm{dt}}=\left(\frac{\mathrm{UA}}{\mathrm{M}_{\mathrm{c}} \mathrm{c}_{\mathrm{pc}}}\right)\left(\mathrm{T}_{\mathrm{f}}-\mathrm{T}_{\mathrm{av}}\right)+\frac{\mathrm{m}_{\mathrm{p}}}{\mathrm{M}_{\mathrm{c}}}\left(\mathrm{T}_{\mathrm{out}}-\overline{\mathrm{T}}_{\mathrm{in}}\right),
$$

donde $\mathrm{M}_{\mathrm{c}}$ es una masa efectiva del refrigerante, $\mathrm{c}_{\mathrm{pc}}$ es el calor específico a presión constante del refrigerante, $\mathrm{m}_{\mathrm{p}}$ es la variación de masa del refrigerante en la vasija por unidad de tiempo, $\mathrm{T}_{\text {out }}$ es la temperatura de salida del refrigerante que se obtiene a partir de la relación aproximada

$$
\mathrm{T}_{\mathrm{av}}=\frac{1}{2}\left(\mathrm{~T}_{\mathrm{out}}+\overline{\mathrm{T}}_{\mathrm{in}}\right)
$$

siendo $\overline{\mathrm{T}}_{\text {in }}$ la temperatura media de entrada del moderador en la vasija. Al tener nuestro modelo dos lazos asimétricos, tales que por el lazo 1 circula el triple de agua que por el lazo 2, supondremos que

$$
\overline{\mathrm{T}}_{\mathrm{in}}=\frac{1}{4}\left(3 \mathrm{~T}_{\mathrm{in} 1}+\mathrm{T}_{\mathrm{in} 2}\right),
$$

donde $\mathrm{T}_{\text {in1 }} \mathrm{y} \mathrm{T}_{\text {in2 }}$ son las temperaturas medias de entrada del agua a la vasija por el lazo 1 y por el lazo 2, respectivamente (véase figura 6.2). Para las temperaturas medias de las ramas frías y calientes del lazo $1, \mathrm{~T}_{\mathrm{cl} 1} \mathrm{y} \mathrm{T}_{\mathrm{hl} 1}$, y del lazo 2, $\mathrm{T}_{\mathrm{cl} 2} \mathrm{y} \mathrm{T}_{\mathrm{hl} 2}$, se tienen las siguientes ecuaciones (véase figura 6.1)

$$
\begin{aligned}
& \frac{\mathrm{dT}_{\mathrm{in} 1}}{\mathrm{dt}}=\frac{1}{\tau_{\mathrm{cl} 1}}\left(\mathrm{~T}_{\mathrm{cl} 1}-\mathrm{T}_{\mathrm{in} 1}\right), \\
& \frac{\mathrm{dT}_{\mathrm{hl} 1}}{\mathrm{dt}}=\frac{1}{\tau_{\mathrm{hl} 1}}\left(\mathrm{~T}_{\mathrm{out}}-\mathrm{T}_{\mathrm{hl} 1}\right), \\
& \frac{\mathrm{dT}_{\mathrm{in} 2}}{\mathrm{dt}}=\frac{1}{\tau_{\mathrm{cl} 2}}\left(\mathrm{~T}_{\mathrm{cl} 2}-\mathrm{T}_{\mathrm{in} 2}\right), \\
& \frac{\mathrm{dT}_{\mathrm{hl} 2}}{\mathrm{dt}}=\frac{1}{\tau_{\mathrm{hl} 2}}\left(\mathrm{~T}_{\mathrm{out}}-\mathrm{T}_{\mathrm{hl} 2}\right),
\end{aligned}
$$


donde $\tau_{\mathrm{cl} 11}, \tau_{\mathrm{h} 11}, \tau_{\mathrm{cl} 2}, \tau_{\mathrm{h} 12}$ son constantes de tiempo de las ramas frías y calientes de cada uno de los dos lazos.

Por último, se plantea el siguiente modelo, muy simplificado (Park1992), para simular el balance energético en cada uno de los dos generadores de vapor

$$
\begin{aligned}
& \mathrm{T}_{\mathrm{cl} 1}=\mathrm{D}_{21} \mathrm{~T}_{\mathrm{s} 1}-\mathrm{D}_{31} \mathrm{~T}_{\mathrm{hl} 1} \\
& \frac{\mathrm{dT}_{\mathrm{s} 1}}{\mathrm{dt}}=-\frac{1}{\tau_{\mathrm{s} 1}}\left(\mathrm{~T}_{\mathrm{s} 1}-\mathrm{T}_{\mathrm{hl} 1}+\mathrm{G}_{1}\right), \\
& \mathrm{T}_{\mathrm{cl} 2}=\mathrm{D}_{22} \mathrm{~T}_{\mathrm{s} 2}-\mathrm{D}_{32} \mathrm{~T}_{\mathrm{h} 12}, \\
& \frac{\mathrm{dT}_{\mathrm{s} 2}}{\mathrm{dt}}=-\frac{1}{\tau_{\mathrm{s} 2}}\left(\mathrm{~T}_{\mathrm{s} 2}-\mathrm{T}_{\mathrm{hl} 2}+\mathrm{G}_{2}\right),
\end{aligned}
$$

donde $D_{21}, D_{31}, G_{1}, D_{22}, D_{32}, G_{2}$ son constantes asociadas con cada uno de los generadores de vapor y $\tau_{\mathrm{s} 1}, \tau_{\mathrm{s} 2}$, son constantes de tiempo para los generadores.

Por último, la correlación que se utilizará para la reactividad, medida en dólares, será de la forma

$$
\rho(t)=\alpha_{f}\left(T_{f}-T_{f}^{0}\right)+\alpha_{a v}\left(T_{a v}-T_{a v}^{0}\right)+\text { rho, }
$$

donde $\mathrm{T}_{\mathrm{f}}^{0}$ y $\mathrm{T}_{\mathrm{av}}^{0}$ son los valores de la temperatura del combustible y del moderador en el estado estacionario, y rho es un término adicional que se utilizará para generar transitorios de inserción de reactividad, como se verá más adelante.

Por tanto, el modelo de reactor PWR planteado se puede rescribir, eliminando las variables dependientes en las ecuaciones anteriores, del siguiente modo

$$
\begin{aligned}
& \frac{\mathrm{dP}}{\mathrm{dt}}=\frac{\beta}{\Lambda}\left(\alpha_{\mathrm{f}}\left(\mathrm{T}_{\mathrm{f}}-\mathrm{T}_{\mathrm{f}}^{0}\right)+\alpha_{\mathrm{av}}\left(\mathrm{T}_{\mathrm{av}}-\mathrm{T}_{\mathrm{av}}^{0}\right)+\mathrm{rho}-1\right) \mathrm{P}+\sum_{\mathrm{i}=1}^{\mathrm{K}} \lambda_{\mathrm{i}} \widetilde{\mathrm{c}}_{\mathrm{i}}, \\
& \frac{\mathrm{d} \widetilde{\mathrm{c}}_{\mathrm{i}}}{\mathrm{dt}}=\frac{\beta_{\mathrm{i}}}{\Lambda} \mathrm{P}-\lambda_{\mathrm{i}} \widetilde{\mathrm{c}}_{\mathrm{i}}, \quad \mathrm{i}=1, \ldots, \mathrm{K}, \\
& \frac{\mathrm{dT}_{\mathrm{f}}}{\mathrm{dt}}=-\mathrm{A}_{1}\left(\mathrm{~T}_{\mathrm{f}}-\mathrm{T}_{\mathrm{av}}\right)+\mathrm{A}_{2} \mathrm{P},
\end{aligned}
$$




$$
\begin{aligned}
& \frac{\mathrm{dT}_{\mathrm{av}}}{\mathrm{dt}}=\mathrm{A}_{3}\left(\mathrm{~T}_{\mathrm{f}}-\mathrm{T}_{\mathrm{av}}\right)+\mathrm{A}_{4}\left(2 \mathrm{~T}_{\mathrm{av}}-\frac{1}{2}\left(3 \mathrm{~T}_{\mathrm{in} 1}+\mathrm{T}_{\mathrm{in} 2}\right)\right) \\
& \frac{\mathrm{dT}_{\mathrm{in} 1}}{\mathrm{dt}}=\frac{1}{\tau_{\mathrm{cl} 1}}\left(\mathrm{D}_{21} \mathrm{~T}_{\mathrm{s} 1}-\mathrm{D}_{31} \mathrm{~T}_{\mathrm{hl} 1}-\mathrm{T}_{\mathrm{in} 1}\right) \\
& \frac{\mathrm{dT}_{\mathrm{hl} 1}}{\mathrm{dt}}=\frac{1}{\tau_{\mathrm{h} 11}}\left(2 \mathrm{~T}_{\mathrm{av}}-\frac{1}{4}\left(3 \mathrm{~T}_{\mathrm{in} 1}+\mathrm{T}_{\mathrm{in} 2}\right)-\mathrm{T}_{\mathrm{hl} 1}\right) \\
& \frac{\mathrm{dT}_{\mathrm{in} 2}}{\mathrm{dt}}=\frac{1}{\tau_{\mathrm{cl} 2}}\left(\mathrm{D}_{22} \mathrm{~T}_{\mathrm{s} 2}-\mathrm{D}_{32} \mathrm{~T}_{\mathrm{hl} 2}-\mathrm{T}_{\mathrm{in} 2}\right), \\
& \frac{\mathrm{dT}_{\mathrm{hl} 2}}{\mathrm{dt}}=\frac{1}{\tau_{\mathrm{hl} 2}}\left(2 \mathrm{~T}_{\mathrm{av}}-\frac{1}{4}\left(3 \mathrm{~T}_{\mathrm{in} 1}+\mathrm{T}_{\mathrm{in} 2}\right)-\mathrm{T}_{\mathrm{hl} 2}\right) \\
& \frac{\mathrm{dT}_{\mathrm{s} 1}}{\mathrm{dt}}=-\frac{1}{\tau_{\mathrm{s} 1}}\left(\mathrm{~T}_{\mathrm{s} 1}-\mathrm{T}_{\mathrm{hl1}}+\mathrm{G}_{1}\right) \\
& \frac{\mathrm{dT}_{\mathrm{s} 2}}{\mathrm{dt}}=-\frac{1}{\tau_{\mathrm{s} 2}}\left(\mathrm{~T}_{\mathrm{s} 2}-\mathrm{T}_{\mathrm{hl} 2}+\mathrm{G}_{2}\right)
\end{aligned}
$$

donde se han introducido las constantes

$$
\mathrm{A}_{1}=\frac{\mathrm{UA}}{\mathrm{M}_{\mathrm{f}} \mathrm{c}_{\mathrm{pf}}}, \quad \mathrm{A}_{2}=\frac{\mathrm{F}}{\mathrm{M}_{\mathrm{f}} \mathrm{c}_{\mathrm{pf}}}, \quad \mathrm{A}_{3}=\frac{\mathrm{UA}}{\mathrm{M}_{\mathrm{c}} \mathrm{c}_{\mathrm{pc}}}, \quad \mathrm{A}_{4}=\frac{\dot{\mathrm{m}}}{\mathrm{M}_{\mathrm{c}}} .
$$

El modelo planteado presenta un gran número de constantes efectivas, por lo que para reducir el número de las mismas se utilizarán las ecuaciones del modelo de estado estacionario, que dan lugar a las siguientes relaciones:

$$
\begin{aligned}
& \mathrm{c}_{\mathrm{i}}^{0}=\frac{\beta_{\mathrm{i}} \mathrm{P}^{0}}{\Lambda \lambda_{\mathrm{i}}}, \\
& \mathrm{A}_{2}=\frac{\mathrm{T}_{\mathrm{f}}^{0}-\mathrm{T}_{\mathrm{av}}^{0}}{\mathrm{P}^{0}} \mathrm{~A}_{1},
\end{aligned}
$$




$$
\begin{aligned}
& \mathrm{A}_{4}=-\frac{\left(\mathrm{T}_{\mathrm{f}}^{0}-\mathrm{T}_{\mathrm{av}}^{0}\right) \mathrm{A}_{3}}{2 \mathrm{~T}_{\mathrm{av}}^{0}-\frac{1}{2}\left(3 \mathrm{~T}_{\mathrm{in} 1}^{0}-\mathrm{T}_{\mathrm{in} 2}^{0}\right),} \\
& \mathrm{D}_{21}=\frac{\mathrm{D}_{31} \mathrm{~T}_{\mathrm{hl} 1}^{0}+\mathrm{T}_{\mathrm{in} 1}^{0}}{\mathrm{~T}_{\mathrm{s} 1}^{0}}, \\
& \mathrm{~T}_{\mathrm{hl} 1}^{0}=2 \mathrm{~T}_{\mathrm{av}}^{0}-\frac{1}{4}\left(3 \mathrm{~T}_{\mathrm{in} 1}^{0}+\mathrm{T}_{\mathrm{in} 2}^{0}\right), \\
& \mathrm{D}_{22}=\frac{\mathrm{D}_{32} \mathrm{~T}_{\mathrm{hl} 2}^{0}+\mathrm{T}_{\mathrm{in} 2}^{0}}{\mathrm{~T}_{\mathrm{s} 2}^{0}}, \\
& \mathrm{~T}_{\mathrm{hl} 2}^{0}=2 \mathrm{~T}_{\mathrm{av}}^{0}-\frac{1}{4}\left(3 \mathrm{~T}_{\mathrm{in} 1}^{0}+\mathrm{T}_{\mathrm{in} 2}^{0}\right), \\
& \mathrm{G}_{1}=\mathrm{T}_{\mathrm{hl1}}^{0}-\mathrm{T}_{\mathrm{s} 1}^{0}, \\
& \mathrm{G}_{2}=\mathrm{T}_{\mathrm{hl} 2}^{0}-\mathrm{T}_{\mathrm{s} 2}^{0},
\end{aligned}
$$

donde las variables del modelo en el estado estacionario se han denotado con el superíndice 0. Usando estas relaciones el modelo de PWR de dos lazos en función de las constantes independientes queda de la forma

$$
\begin{aligned}
& \frac{\mathrm{dP}}{\mathrm{dt}}=\frac{\beta}{\Lambda}\left(\alpha_{\mathrm{f}}\left(\mathrm{T}_{\mathrm{f}}-\mathrm{T}_{\mathrm{f}}^{0}\right)+\alpha_{\mathrm{av}}\left(\mathrm{T}_{\mathrm{av}}-\mathrm{T}_{\mathrm{av}}^{0}\right)+\mathrm{rho}-1\right) \mathrm{P}+\sum_{\mathrm{i}=1}^{\mathrm{K}} \lambda_{\mathrm{i}} \widetilde{\mathrm{c}}_{\mathrm{i}}, \\
& \frac{\mathrm{d} \widetilde{\mathrm{c}}_{\mathrm{i}}}{\mathrm{dt}}=\frac{\beta_{\mathrm{i}}}{\Lambda} \mathrm{P}-\lambda_{\mathrm{i}} \widetilde{\mathrm{c}}_{\mathrm{i}}, \quad \mathrm{i}=1, \ldots, \mathrm{K}, \\
& \frac{\mathrm{dT}_{\mathrm{f}}}{\mathrm{dt}}=-\mathrm{A}_{1}\left(\mathrm{~T}_{\mathrm{f}}-\mathrm{T}_{\mathrm{av}}\right)+\mathrm{A}_{1} \frac{\left(\mathrm{T}_{\mathrm{f}}^{0}-\mathrm{T}_{\mathrm{av}}^{0}\right)}{\mathrm{P}^{0} \mathrm{P},} \\
& \frac{\mathrm{dT}_{\mathrm{av}}}{\mathrm{dt}}=\mathrm{A}_{3}\left(\mathrm{~T}_{\mathrm{f}}-\mathrm{T}_{\mathrm{av}}\right)+\mathrm{A}_{3} \frac{\left(\mathrm{T}_{\mathrm{f}}^{0}-\mathrm{T}_{\mathrm{av}}^{0}\right)}{2 \mathrm{~T}_{\mathrm{av}}^{0}-\frac{1}{2}\left(3 \mathrm{~T}_{\mathrm{in} 1}^{0}+\mathrm{T}_{\mathrm{in} 2}^{0}\right)}\left(2 \mathrm{~T}_{\mathrm{av}}-\frac{1}{2}\left(3 \mathrm{~T}_{\mathrm{in} 1}+\mathrm{T}_{\mathrm{in} 2}\right)\right), \\
& \frac{\mathrm{dT} \mathrm{in} 1}{\mathrm{dt}}=\frac{1}{\tau_{\mathrm{cl} 1}}\left(\frac{\mathrm{D}_{31} \mathrm{~T}_{\mathrm{hl} 1}^{0}+\mathrm{T}_{\mathrm{in} 1}^{0}}{\mathrm{~T}_{\mathrm{s} 1}^{0}} \mathrm{~T}_{\mathrm{s} 1}-\mathrm{D}_{31} \mathrm{~T}_{\mathrm{hl1}}-\mathrm{T}_{\mathrm{in} 1}\right),
\end{aligned}
$$




$$
\begin{aligned}
& \frac{\mathrm{dT}_{\mathrm{hl1}}}{\mathrm{dt}}=\frac{1}{\tau_{\mathrm{hl} 1}}\left(2 \mathrm{~T}_{\mathrm{av}}-\frac{1}{4}\left(3 \mathrm{~T}_{\mathrm{in} 1}+\mathrm{T}_{\mathrm{in} 2}\right)-\mathrm{T}_{\mathrm{hl} 1}\right), \\
& \frac{\mathrm{dT}_{\mathrm{in} 2}}{\mathrm{dt}}=\frac{1}{\tau_{\mathrm{cl} 2}}\left(\frac{\mathrm{D}_{32} \mathrm{~T}_{\mathrm{hl} 2}^{0}+\mathrm{T}_{\mathrm{in} 2}^{0}}{\mathrm{~T}_{\mathrm{s} 2}^{0}} \mathrm{~T}_{\mathrm{s} 2}-\mathrm{D}_{32} \mathrm{~T}_{\mathrm{hl} 2}-\mathrm{T}_{\mathrm{in} 2}\right), \\
& \frac{\mathrm{dT}_{\mathrm{hl} 2}}{\mathrm{dt}}=\frac{1}{\tau_{\mathrm{h} 12}}\left(2 \mathrm{~T}_{\mathrm{av}}-\frac{1}{4}\left(3 \mathrm{~T}_{\mathrm{in} 1}+\mathrm{T}_{\mathrm{in} 2}\right)-\mathrm{T}_{\mathrm{hl} 2}\right), \\
& \frac{\mathrm{dT}_{\mathrm{s} 1}}{\mathrm{dt}}=-\frac{1}{\tau_{\mathrm{s} 1}}\left(\mathrm{~T}_{\mathrm{s} 1}-\mathrm{T}_{\mathrm{hl} 1}+\mathrm{T}_{\mathrm{hl} 1}^{0}-\mathrm{T}_{\mathrm{s} 1}^{0}\right), \\
& \frac{\mathrm{dT}_{\mathrm{s} 2}}{\mathrm{dt}}=-\frac{1}{\tau_{\mathrm{s} 2}}\left(\mathrm{~T}_{\mathrm{s} 2}-\mathrm{T}_{\mathrm{h} 12}+\mathrm{T}_{\mathrm{h} 12}^{0}-\mathrm{T}_{\mathrm{s} 2}^{0}\right) .
\end{aligned}
$$

\subsection{Ajuste y optimización de parámetros}

\subsubsection{Ajuste de parámetros en estado estacionario.}

La primera acción a desarrollar antes de abordar el problema de optimización de parámetros consiste en una estimación de los mismos en función de las características de la planta a simular. Como ya hemos comentado la instalación de referencia utilizada en este estudio es la proporcionada por el fichero de entrada "typpwr.i" que constituye uno de los casos ejemplo suministrados en el paquete de instalación del RELAP5. Por tanto, los datos para la estimación inicial de las constantes del modelo reducido se basarán en la información suministrada por esta nodalización mediante la versión RELAP5/Mod3.2fg.

Para simular la evolución de la potencia en el núcleo de reactor mediante el modelo de RELAP que se ha tomado de referencia, mostrado en el Apéndice 3, éste utiliza las ecuaciones de la cinética puntual con seis grupos de precursores. Estas ecuaciones son las mismas que utiliza el modelo reducido que se ha desarrollado en el apartado anterior, por tanto las constantes asociadas a estas ecuaciones se supondrán fijas con los valores presentados en la tabla 6.1 (Park1992). 


\begin{tabular}{lllllll}
\hline$\beta$ & $\beta_{1}$ & $\beta_{2}$ & $\beta_{3}$ & $\beta_{4}$ & $\beta_{5}$ & $\beta_{6}$ \\
0.007108 & 0.000216 & 0.001416 & 0.001349 & 0.00218 & 0.00095 & 0.000322 \\
\hline$\Lambda(\mathrm{s})$ & $\lambda_{1}$ & $\lambda_{2}$ & $\lambda_{3}$ & $\lambda_{4}$ & $\lambda_{5}$ & $\lambda_{6}$ \\
$2.3910^{-5}$ & 0.0125 & 0.0308 & 0.1152 & 0.3109 & 1.24 & 3.3287 \\
\hline
\end{tabular}

Tabla 6.1: Parámetros de las ecuaciones de la cinética puntual.

En primer lugar, para comparar el comportamiento del modelo reducido con el del RELAP se ha tomado como variable de estudio la evolución de la potencia. Ejecutando el modelo de RELAP tal como aparece en el Apéndice 3, se obtiene la evolución de la potencia que se muestra en la figura 6.3. En esta figura se observa que en los primeros instantes de tiempo el sistema no se encuentra estacionario puesto que la potencia oscila, hasta estabilizarse aproximadamente a los 50 segundos. Por esta razón, los valores de las variables del modelo reducido en el estado estacionario se tomarán iguales a los valores calculados por el RELAP a los 50 segundos.

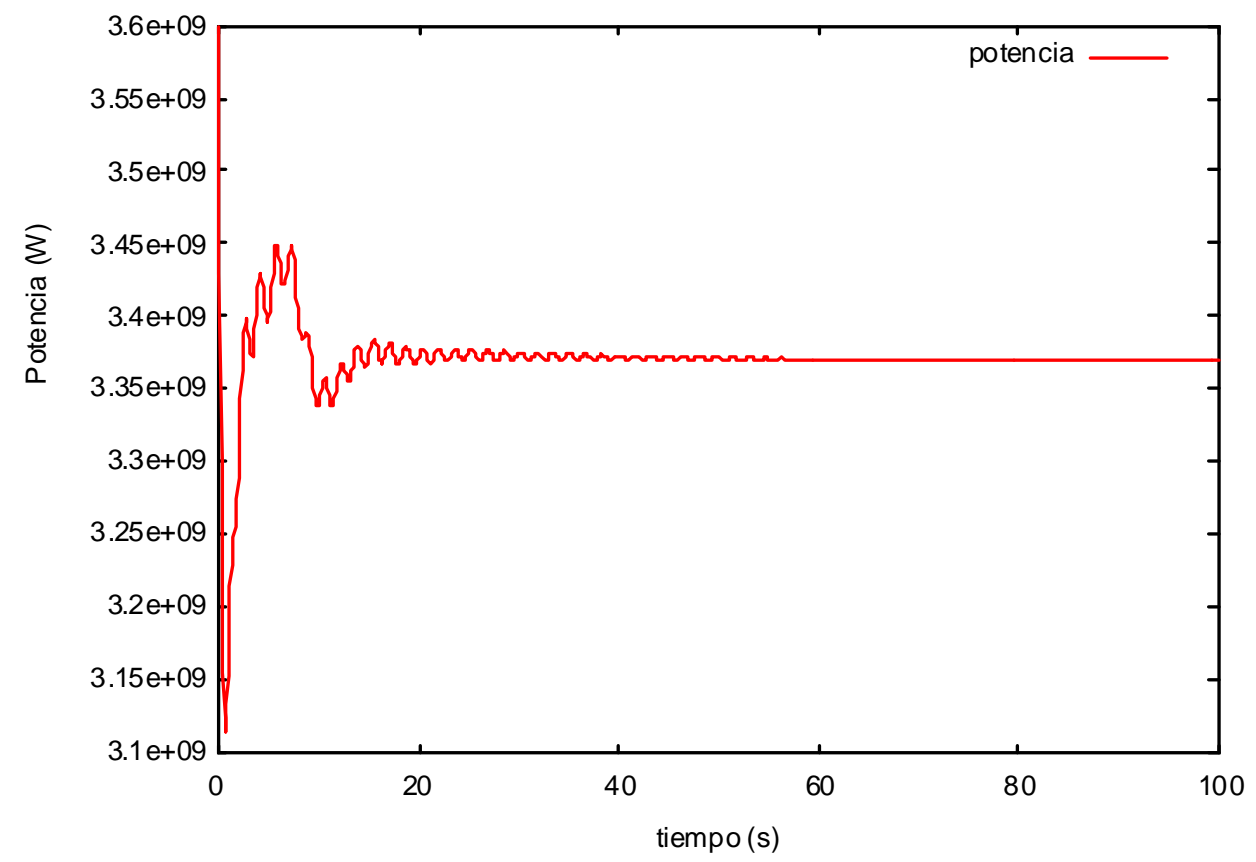

Figura 6.3: Evolución de la potencia con RELAP5.

El valor de la potencia, $\mathrm{P}$, generada en el núcleo del reactor es un valor proporcionado directamente por la variable rktpow del RELAP. La temperatura media del combustible, $\mathrm{T}_{\mathrm{f}}$, se calcula mediante una variable de control en la que se hace la media de la distribución radial de temperaturas en cada división axial de la estructura de calor, para después promediar las temperaturas medias de cada nivel axial. La temperatura media del moderador, $\mathrm{T}_{\mathrm{av}}$, se obtiene mediante un control en el se calcula la media de las 
temperaturas del moderador en el primer y en el último volumen del núcleo. La temperatura de entrada al vasija desde los lazos, $\mathrm{T}_{\mathrm{in} 1}, \mathrm{~T}_{\mathrm{in} 2}$, se obtienen directamente de las temperaturas del fluido en el último volumen de los lazos antes de entrar a la vasija. Las temperaturas del las ramas calientes, $\mathrm{T}_{\mathrm{hl1}}, \mathrm{T}_{\mathrm{hl} 2}$, se toman también directamente como las temperaturas del agua en cada uno de los lazos del circuito primario justo antes de entrar en los tubos de los generadores de vapor. Finalmente, las temperaturas del secundario de los generadores de vapor, $\mathrm{T}_{\mathrm{s} 1}, \mathrm{~T}_{\mathrm{s} 2}$, se han tomado las temperaturas del agua en el volumen superior de cada uno de los generadores de vapor.

Teniendo en cuenta los datos proporcionados por el RELAP y las relaciones (6.38) y (6.39) los valores tomados del modelo reducido para el estado estacionario son los que se muestran en la tabla 6.2.

\begin{tabular}{llll}
\hline $\mathrm{P}^{0}(\mathrm{MW})$ & $\mathrm{T}_{\mathrm{f}}^{0}\left({ }^{\mathrm{o}} \mathrm{K}\right)$ & $\mathrm{T}_{\mathrm{av}}^{0}\left({ }^{\mathrm{o}} \mathrm{K}\right)$ & $\mathrm{T}_{\mathrm{in} 1}^{0}\left({ }^{\mathrm{o}} \mathrm{K}\right)$ \\
3378 & 823 & 570 & 551 \\
\hline $\mathrm{T}_{\mathrm{in} 2}^{0}\left({ }^{\mathrm{o}} \mathrm{K}\right)$ & $\mathrm{T}_{\mathrm{h} 11}^{0}=\mathrm{T}_{\mathrm{h} 12}^{0}\left({ }^{\circ} \mathrm{K}\right)$ & $\mathrm{T}_{\mathrm{s} 1}^{0}\left({ }^{\mathrm{o}} \mathrm{K}\right)$ & $\mathrm{T}_{\mathrm{s} 2}^{0}\left({ }^{\mathrm{o}} \mathrm{K}\right)$ \\
551 & 589 & 540 & 535 \\
\hline
\end{tabular}

Tabla 6.2: Valores de las variables del modelo reducido en el estacionario.

\subsubsection{Búsqueda y optimización de parámetros ante transitorios de inserción de reactividad.}

Para el ajuste de algunos de los parámetros del modelo reducido se realizará un transitorio de inserción de reactividad que correspondería a un movimiento en las barras de control realizado por el operador. La simulación de este transitorio mediante RELAP5 se ha realizado a través de una tabla de reactividad controlada por un trip. De este modo, el trip se acciona a los 50 segundos y el modelo revisa a partir de este momento la tabla que regula la inserción de reactividad. En esta tabla la reactividad permanece nula hasta los 52 segundos de transitorio, a partir de los cuales la reactividad sube linealmente hasta que a los 56 segundos es de 0.1 dólares, manteniéndose este valor después de este instante.

Los primeros 50 segundos del transitorio simulados mediante RELAP no se van a tomar en consideración en los procesos de búsqueda y optimización de parámetros que se van a llevar a cabo, puesto que para el modelo reducido las condiciones del estacionario se toman en este instante. En total se considerarán 20 segundos de transitorio a partir del tiempo en el que se ha tomado el estacionario para realizar la optimización de los parámetros. 
Para simular el transitorio de inserción de reactividad con el modelo reducido se hace que el término rho de la expresión (6.40) sea de la forma

$$
\text { rho }=\left\{\begin{array}{cc}
0 & \text { si } 0 \leq \mathrm{t} \leq 2 \\
\frac{0.1}{4}(\mathrm{t}-2) & \text { si } 2 \leq \mathrm{t} \leq 6 \\
0.1 & \text { si } 6 \leq \mathrm{t} \leq 20
\end{array}\right.
$$

donde $t$ representa el tiempo en segundos.

Para la integración numérica del modelo reducido se ha hecho uso de la rutina cvdense del paquete CVODE, que es una librería escrita en lenguaje $\mathrm{C}$ con distintos programas para la integración de sistemas de ecuaciones diferenciales (Cohen1995), que engloba las librerías VODE y VODPK escritas inicialmente en lenguaje FORTRAN (Brown1989a; Brown1989b). Se han utilizado para la integración numérica las opciones BDF, que implica la utilización de un método en diferencias hacia atrás, para la resolución de sistemas de ecuaciones diferenciales rígidos, y la opción Newton, que utiliza el método de Newton para la resolución de los sistemas de ecuaciones no lineales que aparecen (Cohen1995). Se han simulado 20 segundos de transitorio, fijando un paso máximo para la integración numérica de 0.001 segundos e imprimiendo los resultados cada 0.5 segundos.

Los valores iniciales para los parámetros se han estimado a partir de los datos del modelo del RELAP5 presentado en el Apéndice 3, y de las referencias (Park1992; Park1993; March-Leuba1986), los cuales se presentan en la tabla 6.3.

\begin{tabular}{llllll}
\hline$\alpha_{\mathrm{f}}$ & $\alpha_{\mathrm{av}}$ & $\mathrm{A}_{1}(1 / \mathrm{seg})$ & $\mathrm{A}_{3}(1 / \mathrm{seg})$ & $\mathrm{D}_{31}(\mathrm{seg})$ & $\mathrm{D}_{32}(\mathrm{seg})$ \\
-0.108032 & -0.00607 & 0.166 & 0.006 & -0.3 & -0.3 \\
\hline$\tau_{\mathrm{cl} 1}(\mathrm{seg})$ & $\tau_{\mathrm{c} 12}(\mathrm{seg})$ & $\tau_{\mathrm{h} 11}(\mathrm{seg})$ & $\tau_{\mathrm{hl} 2}(\mathrm{seg})$ & $\tau_{\mathrm{s} 1}(\mathrm{seg})$ & $\tau_{\mathrm{s} 2}(\mathrm{seg})$ \\
2 & 7 & 1.5 & 5 & 4 & 11 \\
\hline
\end{tabular}

Tabla 6.3: Valores iniciales para los parámetros del modelo reducido.

El transitorio de inserción de reactividad provoca una variación brusca de la potencia generada en el núcleo del reactor, por lo que en un principio se ha considerado que esta variable es una buena referencia para optimizar algunos de los parámetros efectivos del sistema de ecuaciones que define el modelo reducido de PWR.

Tras realizar un análisis de sensibilidad de los parámetros para determinar cuáles son los más influyentes en la evolución de la potencia se han considerado cuatro parámetros, que se describen seguidamente, para su optimización. En la ecuación (6.28), que define la variación de la potencia del núcleo del reactor con el tiempo aparecen los factores $\alpha_{\mathrm{f}}$ 
y $\alpha_{\mathrm{av}}$, que determinan las contribuciones de la temperatura del combustible y del moderador a la reactividad, respectivamente. En los modelos detallados, estos factores dependen de las temperaturas locales, sin embargo, en los modelos reducidos se toma un valor efectivo para todas las zonas del núcleo, con lo cual no van a ser iguales a los utilizados en el modelo detallado. Además, en las ecuaciones que definen el balance térmico entre el combustible y el agua del núcleo del reactor, ecuaciones (6.30) y (6.31), aparecen las constantes $\mathrm{A}_{1}$ y $\mathrm{A}_{3}$ definidas en función de valores efectivos de variables como el área, la masa del combustible o la masa del refrigerante. Para la optimización de los demás parámetros del modelo habría que considerar otro tipo de transitorios y tomar otras variables, como por ejemplo la temperatura en los lazos, como variables de referencia.

Por tanto, la primera optimización llevada a cabo en este estudio ha tenido en cuenta los parámetros $\alpha_{\mathrm{f}}, \alpha_{\mathrm{av}}, \mathrm{A}_{1}$ y $\mathrm{A}_{3}$ del modelo reducido tomando como referencia la evolución de la potencia en el núcleo calculada mediante RELAP5/Mod3.2fg. Se ha utilizando la metodología expuesta en los capítulos anteriores, que hace uso del método MDS para la optimización de los parámetros.

En la optimización que nos ocupa cada parámetro ha sido representado por una variable en el MDS, sin que exista ningún tipo de relación entre ellas que deban cumplirse. Además, no es necesario el escalado de las mismas puesto que los órdenes de magnitud de todas ellas son similares. En la tabla 6.4 se presenta el símplice inicial utilizado para realizar la optimización, los valores óptimos alcanzados para cada uno de los parámetros y el número de evaluaciones de la función objetivo que ha sido necesario para alcanzar esta solución.

\begin{tabular}{llllll}
\hline & $\alpha_{\mathrm{f}}$ & $\alpha_{\mathrm{av}}$ & $\mathrm{A}_{1}$ & $\mathrm{~A}_{3}$ & \\
\hline Símplice inicial & -0.00607 & -0.108032 & 0.166 & 0.006 & \\
& -0.01607 & -0.108032 & 0.166 & 0.006 & \\
& -0.00607 & -0.208032 & 0.166 & 0.006 & \\
& -0.00607 & -0.208032 & 0.266 & 0.006 & \\
\hline Óptimo & -0.00607 & -0.208032 & 0.166 & 0.016 & \\
\hline Número de evaluaciones & -0.016328 & -0.092659 & 0.315999 & 0.013187 & \\
\hline
\end{tabular}

Tabla 6.4: Valores de los parámetros óptimos tomando la potencia como referencia.

El modelo reducido nos da la evolución de la potencia en el núcleo presentada en la figura 6.4 con los valores de los parámetros proporcionados por la optimización, en la que se compara esta evolución con la de la potencia calculada por el RELAP5/Mod3.2 y que es la que se ha tomado como referencia. 


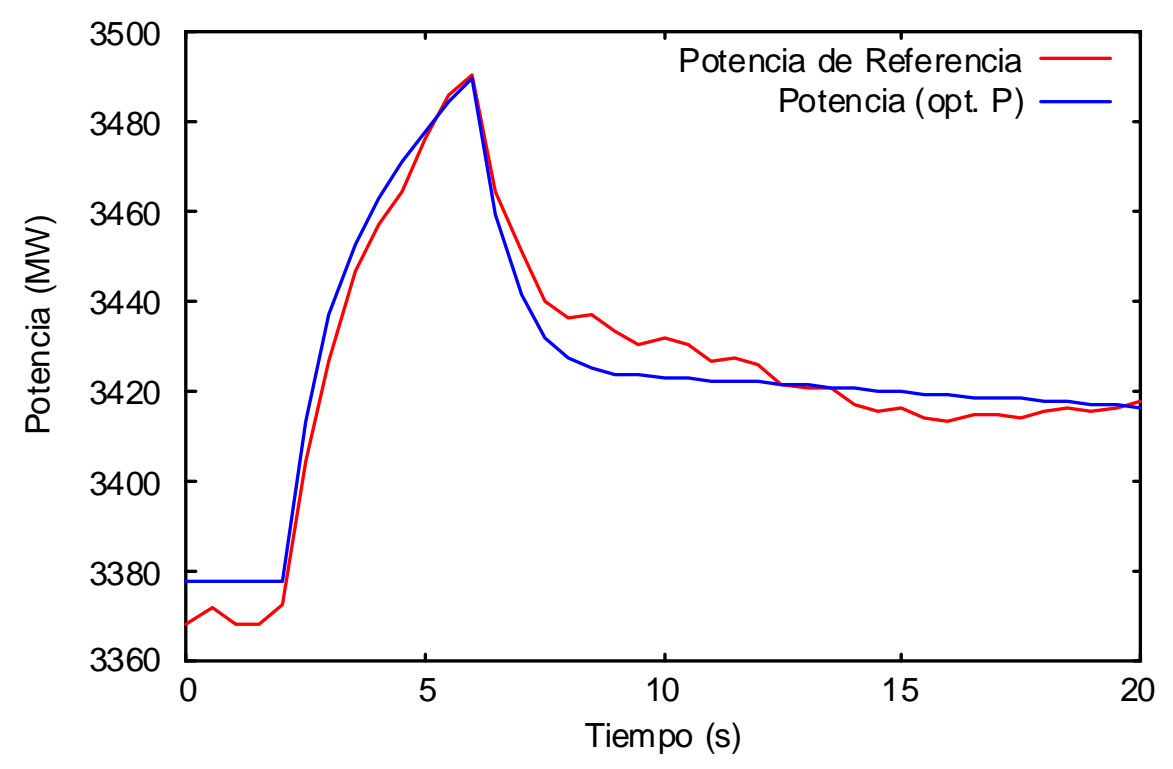

Figura 6.4: Comparación de las evoluciones de las potencias tomando como referencia la potencia.

En esta gráfica se observa que el modelo reducido utilizando los parámetros obtenidos como resultado de la optimización proporciona una evolución para la potencia similar a la proporcionada por el RELAP5. Sin embargo, como el objetivo del estudio es proporcionar información global sobre las variables más importantes de una planta, es necesario saber si los valores óptimos de los parámetros proporcionan resultados aceptables para las demás variables del modelo. En concreto, la figura 6.5 muestra la evolución de la temperatura del combustible proporcionada por el modelo reducido con los parámetros óptimos de la tabla 6.3 junto con la temperatura calculada mediante RELAP5.

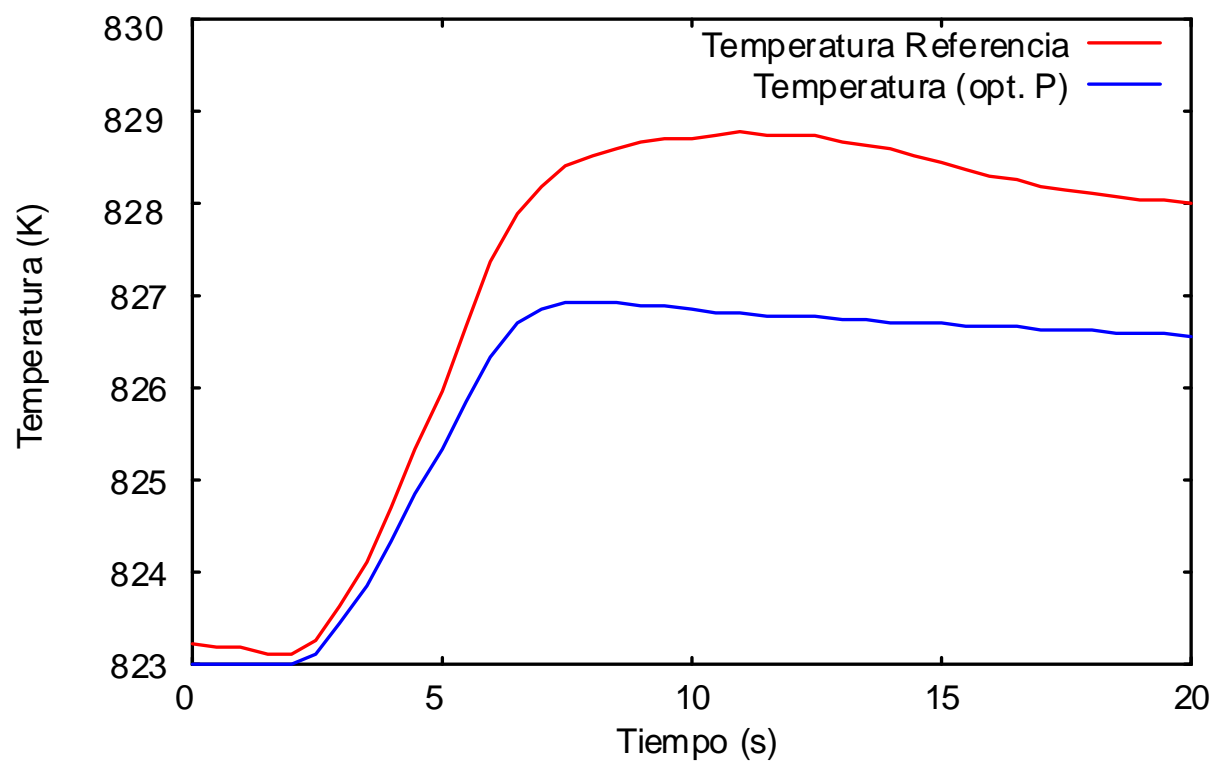

Figura 6.5: Comparación de las evoluciones de la temperatura del combustible tomando como referencia la potencia. 
En la gráfica anterior se muestra una diferencia de unos grados entre las evoluciones de las temperaturas del combustible calculadas con el modelo reducido y con RELAP5. Esto hace pensar que si se toma como referencia la evolución de la temperatura del combustible los valores óptimos de los parámetros del modelo reducido pueden cambiar. En efecto, al realizar la optimización de los parámetros anteriores utilizando como referencia la temperatura del combustible se obtienen los valores mostrados en la tabla 6.5.

\begin{tabular}{llllll}
\hline & $\alpha_{\mathrm{f}}$ & $\alpha_{\mathrm{av}}$ & $\mathrm{A}_{1}$ & $\mathrm{~A}_{3}$ & \\
\hline Símplice inicial & -0.00607 & -0.108032 & 0.166 & 0.006 & \\
& -0.01607 & -0.108032 & 0.166 & 0.006 & \\
& -0.00607 & -0.208032 & 0.166 & 0.006 & \\
& -0.00607 & -0.208032 & 0.266 & 0.006 & \\
& -0.00607 & -0.208032 & 0.166 & 0.016 & \\
\hline Óptimo & -0.01607 & -0.070711 & 0.16599 & 0.003499 & \\
\hline Número de evaluaciones & & & & & 135 \\
\hline
\end{tabular}

Tabla 6.5: Valores de los parámetros óptimos tomando la temperatura del combustible como referencia.

Para los valores óptimos de los parámetros a los que ha llegado el método MDS, se han calculado las evoluciones de la potencia y de la temperatura del combustible que calcula el modelo reducido de reactor, las cuales se muestran comparadas con sus referencias, obtenidas con RELAP5, se presentan en las figuras 6.6 y 6.7 , respectivamente.

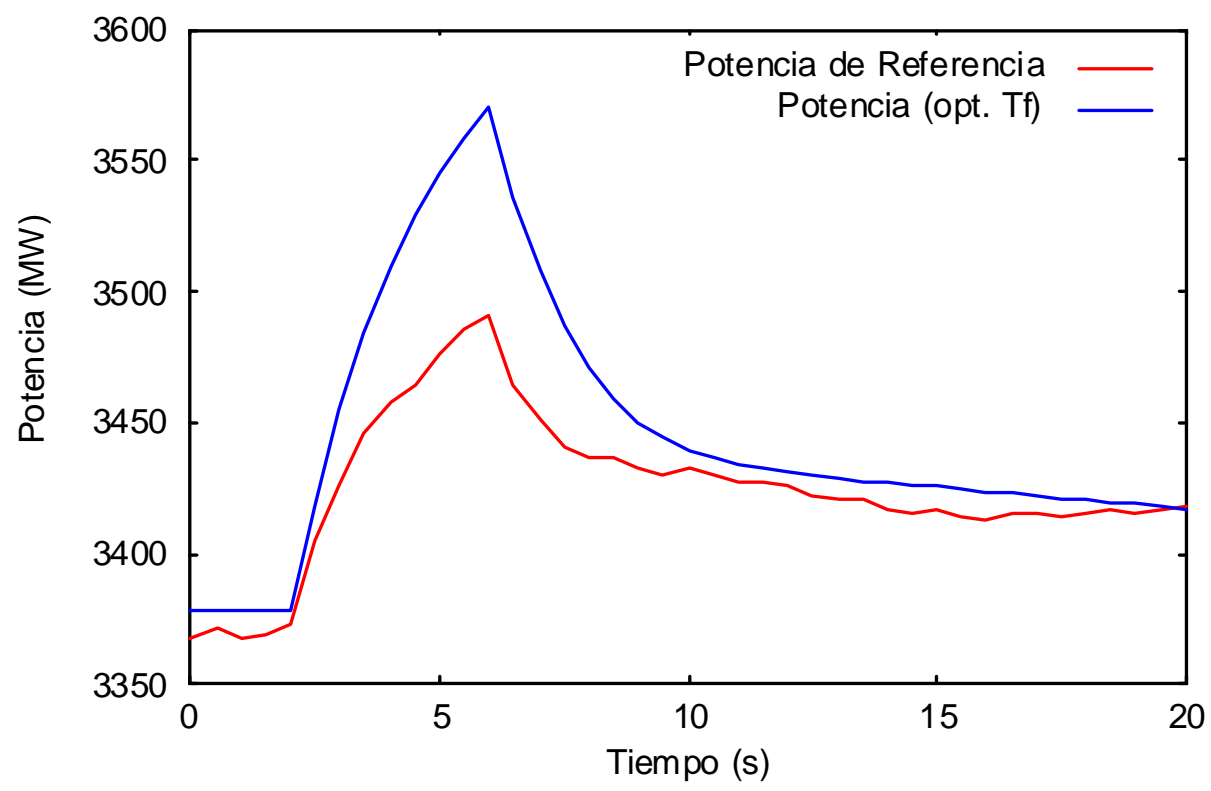

Figura 6.6: Comparación de las evoluciones de la potencia tomando como referencia la temperatura del combustible. 


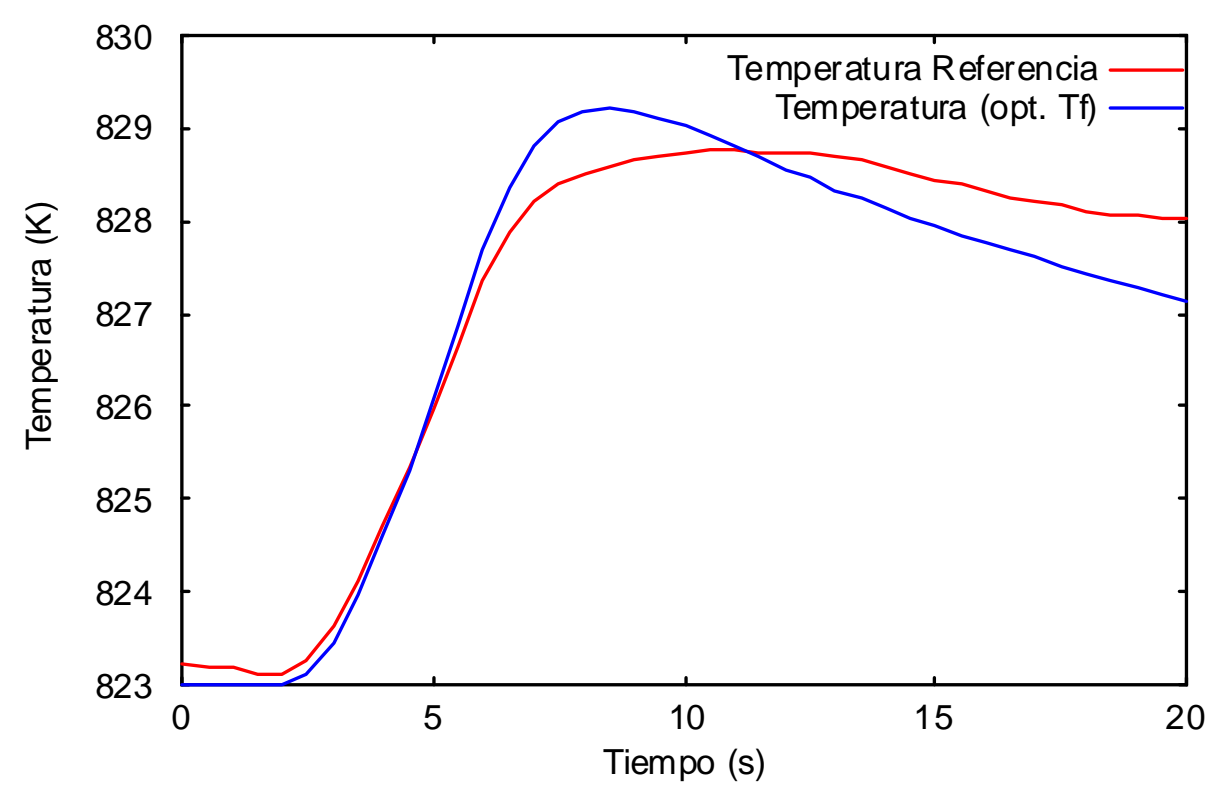

Figura 6.7: Comparación de las evoluciones de la temperatura del combustible tomando como referencia la temperatura del combustible.

En estas figuras se observa que, con los nuevos parámetros se mejora la evolución de la temperatura del combustible y se empeora la curva de la potencia generada en el núcleo del reactor. Podíamos llegar a pensar que para la optimización conjunta de las dos variables es posible utilizar ambas referencias para construir una función objetivo de la forma

$\mathrm{f}=\operatorname{err}_{\mathrm{P}}+\mathrm{w} \cdot \operatorname{err}_{\mathrm{Tf}}$

donde $\operatorname{err}_{\mathrm{P}}$ es el error relativo asociado a la evolución de la potencia, $\operatorname{err}_{\mathrm{Tf}}$ es el error relativo asociado a la evolución de la temperatura del combustible y w es un factor de ponderación que se ha de elegir, por ejemplo, en el caso que nos ocupa, de forma que las contribuciones de los dos errores sean comparables. En este caso particular, se ha elegido un $\mathrm{v}$ alor $\mathrm{w}=10$, y tras la optimización se ha llegado a los valores que se presentan en la Tabla 6.6.

\begin{tabular}{llllll}
\hline & $\alpha_{\mathrm{f}}$ & $\alpha_{\mathrm{av}}$ & $\mathrm{A}_{1}$ & $\mathrm{~A}_{3}$ & \\
\hline Símplice inicial & -0.00607 & -0.108032 & 0.166 & 0.006 & \\
& -0.01607 & -0.108032 & 0.166 & 0.006 & \\
& -0.00607 & -0.208032 & 0.166 & 0.006 & \\
& -0.00607 & -0.208032 & 0.266 & 0.006 & \\
\hline Óptimo & -0.00607 & -0.208032 & 0.166 & 0.016 & \\
\hline Número de evaluaciones & -0.01607 & -0.1051859 & 0.27225 & 0.0022492 & \\
\hline
\end{tabular}

Tabla 6.6: Valores de los parámetros óptimos tomando dos referencias. 
Las evoluciones de la potencia del reactor y de la temperatura del combustible proporcionadas por el modelo reducido utilizando los nuevos valores optimizados para los parámetros se muestran en las gráficas 6.8 y 6.9 , respectivamente.

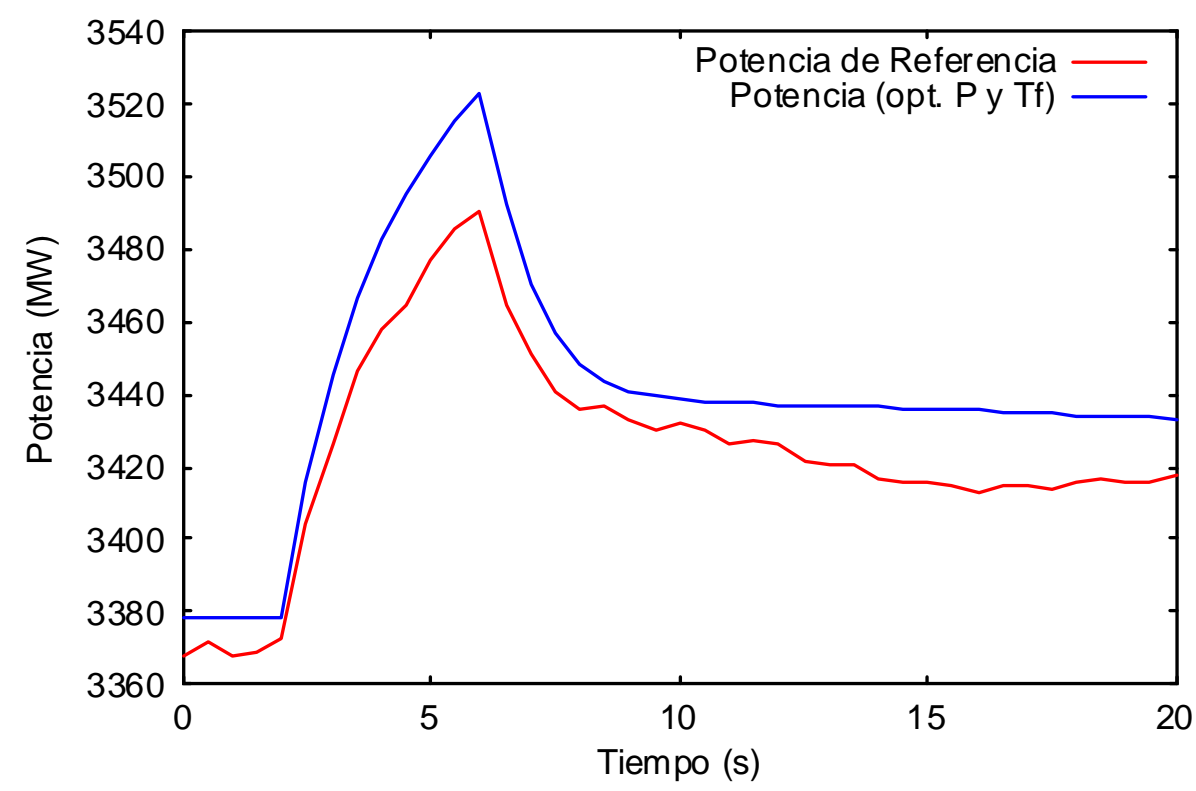

Figura 6.8: Comparación de las evoluciones de la potencia tomando la potencia y la temperatura del combustible como referencias.

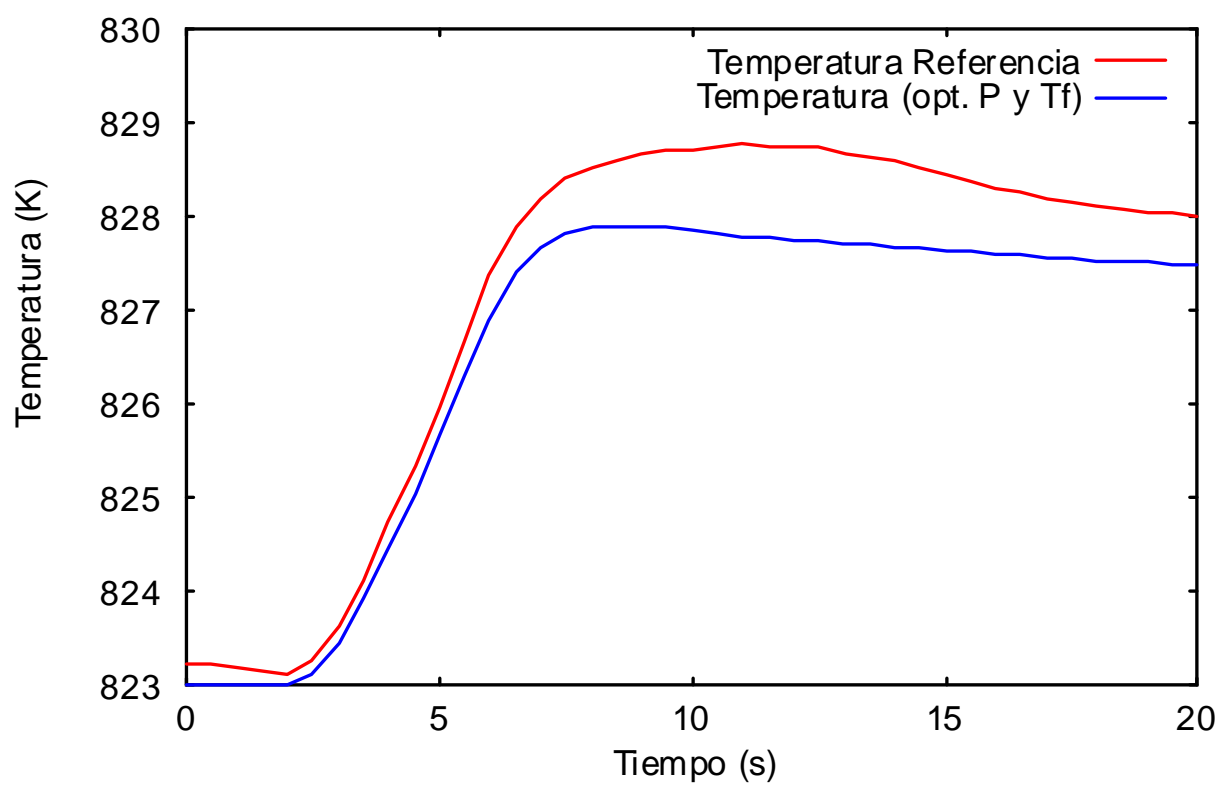

Figura 6.9: Comparación de las evoluciones de la temperatura tomando la potencia y la temperatura del combustible como referencias. 
En las gráficas anteriores se aprecia que los resultados obtenidos se encuentran entre los valores extremos calculados al considerar una sola referencia. En la tabla 6.7 se muestran los valores de los errores para los parámetros óptimos según la referencia que se ha utilizado.

\begin{tabular}{lcc}
\hline & Error Potencia & Error Temperatura \\
\hline Referencia Potencia & 0.0012979 & 0.0013937 \\
\hline Referencia Temperatura & 0.0091444 & 0.0001578 \\
\hline Ambas Referencias & 0.0059060 & 0.0002700 \\
\hline
\end{tabular}

Tabla 6.7: Errores calculados en cada optimización.

En la tabla 6.7 se comprueba, que para el caso de considerar como referencia la potencia en el núcleo, el error cometido en el cálculo de la potencia es pequeño, sin embargo el error entre la temperatura del combustible calculada y la de referencia es de un orden de magnitud mayor que en los demás casos. De la misma manera, si analizamos los errores cometidos en las variables de potencia y temperatura cuando se utilizan los parámetros de la optimización utilizando como referencia la temperatura, se observa que para esta variable el error es muy bueno, sin embargo la potencia que proporciona este cálculo presenta un error bastante superior al del caso anterior. Entre estos dos casos se sitúan los cálculos realizados con los parámetros óptimos si se utilizan ambas referencias, que para cualquiera de dos variables consideradas proporciona unos cálculos cuyos errores se encuentran entre los extremos proporcionados por las optimizaciones utilizando una sola referencia.

Por esta razón, si lo que se pretende en encontrar unos valores de los parámetros del modelo reducido de reactor que nos proporcione unos cálculos aproximados del comportamiento general de la plana ante un transitorio determinado, es aconsejable la utilización en la optimización de varias referencias, y son éstos los datos que se han tomado para las siguientes comprobaciones.

En efecto, además de la posibilidad de contemplar varias referencias en la optimización, es deseable que los parámetros óptimos sean aplicables a cualquier otro transitorio similar. Se ha considerado la posibilidad de cambiar el transitorio para comprobar los valores óptimos de los parámetros en esta situación. De este modo se ha realizado el cálculo para una inserción negativa de reactividad de 0.1 dólares. La función que determina la inserción de reactividad viene dada por 


$$
\text { rho }=\left\{\begin{array}{cc}
0 & \text { si } 0 \leq t \leq 2 \\
-\frac{0.1}{4}(t-2) & \text { si } 2 \leq t \leq 6 \\
-0.1 & \text { si } 6 \leq t \leq 20
\end{array}\right.
$$

donde t es el tiempo expresado en segundos.

De la misma forma que en el caso anterior se ha generado la referencia con el RELAP5 considerando como condiciones de inicio del transitorio los 50 segundos y considerando un tiempo de simulación total de 20 segundos. Los resultados obtenidos se muestran en las figuras 6.10 y 6.11 .

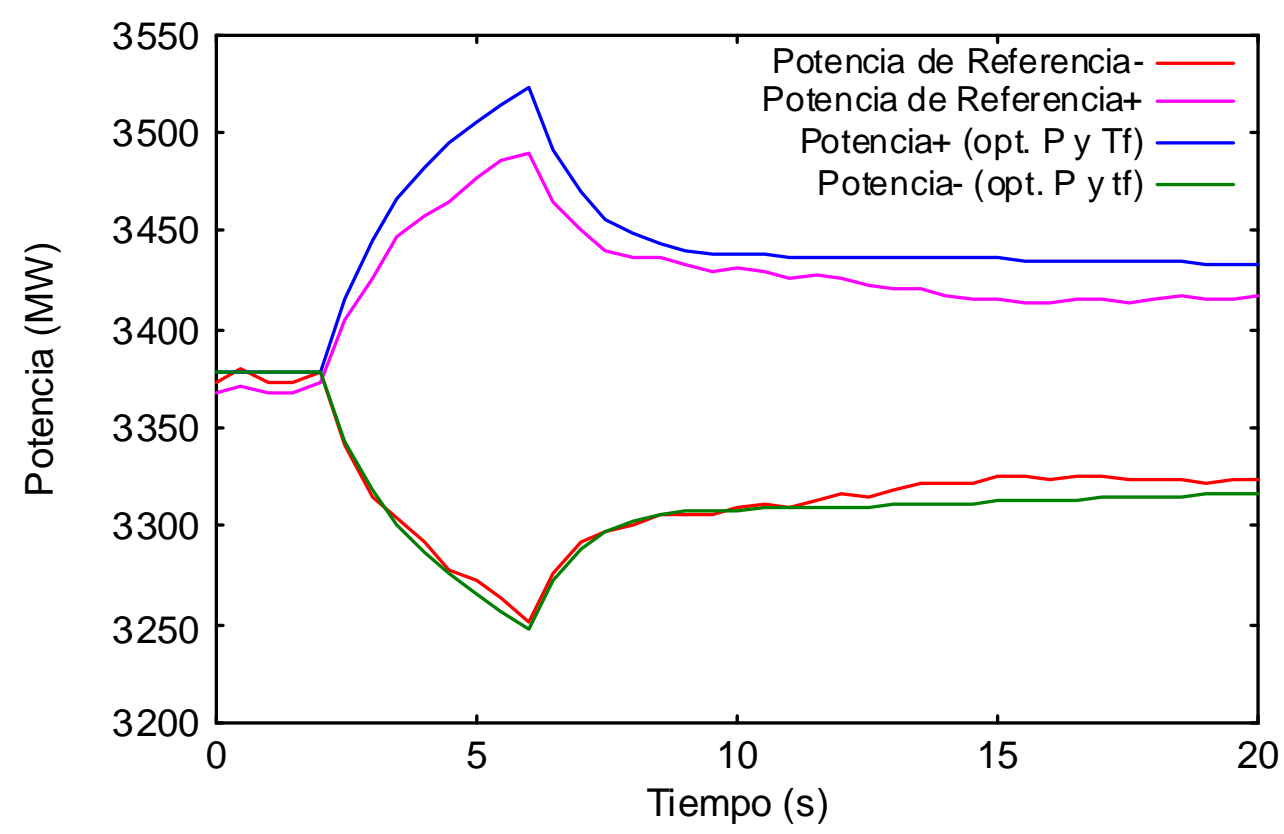

Figura 6.10: Comparación de las evoluciones de la potencia para los dos transitorios.

La figura 6.10 muestra la evolución de la potencia que calcula el modelo reducido para las inserciones de reactividad positiva y negativa comparadas con sus referencias respectivas. Para calcular la evolución de la potencia ante un transitorio de inserción negativa de reactividad se han tomado los valores de los parámetros óptimos del transitorio de inserción positiva en el que se han considerado la evolución de la potencia en el núcleo y la temperatura del combustible como referencias simultáneamente. 


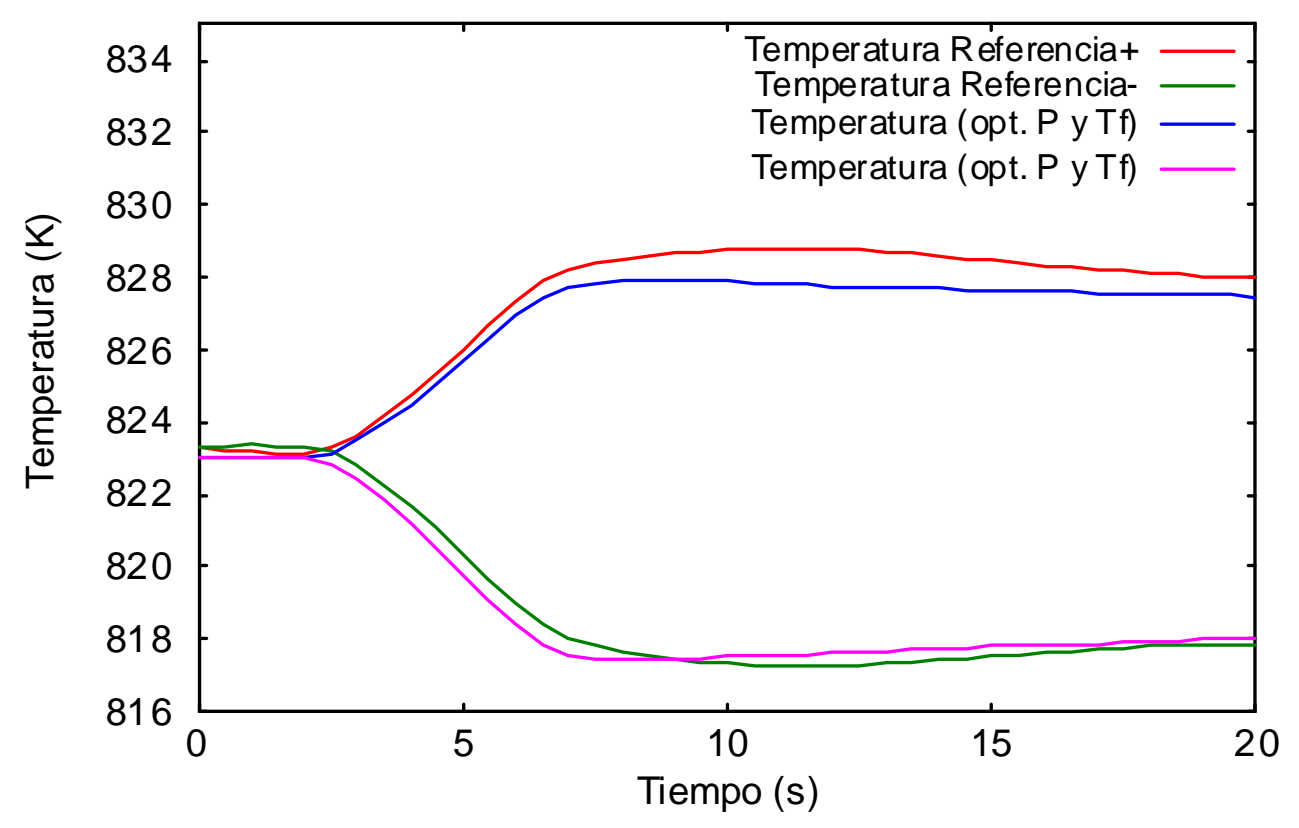

Figura 6.11: Comparación de las evoluciones de la temperatura del combustible para los dos transitorios.

Se observa que los parámetros óptimos para la inserción positiva permiten simular con bastante precisión el transitorio asociado a la inserción negativa de reactividad. No obstante se ha realizado el caso de optimización de la inserción negativa de reactividad con el fin de determinar tanto los valores óptimos de los parámetros en este caso como los errores cometidos al utilizar unos determinados parámetros. Los parámetros óptimos a los que se ha llegado en la optimización de la inserción negativa de reactividad se encuentran en la tabla 6.8 .

\begin{tabular}{llllll}
\hline & $\alpha_{\mathrm{f}}$ & $\alpha_{\mathrm{av}}$ & $\mathrm{A}_{1}$ & $\mathrm{~A}_{3}$ & \\
\hline Símplice inicial & -0.00607 & -0.108032 & 0.166 & 0.006 & \\
& -0.01607 & -0.108032 & 0.166 & 0.006 & \\
& -0.00607 & -0.208032 & 0.166 & 0.006 & \\
& -0.00607 & -0.208032 & 0.266 & 0.006 & \\
\hline Óptimo & -0.00607 & -0.208032 & 0.166 & 0.016 & \\
\hline Número de evaluaciones & -0.016695 & -0.100503 & 0.2425 & 0.001625 & \\
\hline
\end{tabular}

Tabla 6.8: Valores de los parámetros óptimos de la inserción negativa de reactividad tomando dos referencias.

Con los valores de los parámetros de la tabla 6.8 las evoluciones de la potencia y de la temperatura del combustible calculadas por el modelo reducido son las que se muestran en las figuras 6.12 y 6.13 . 


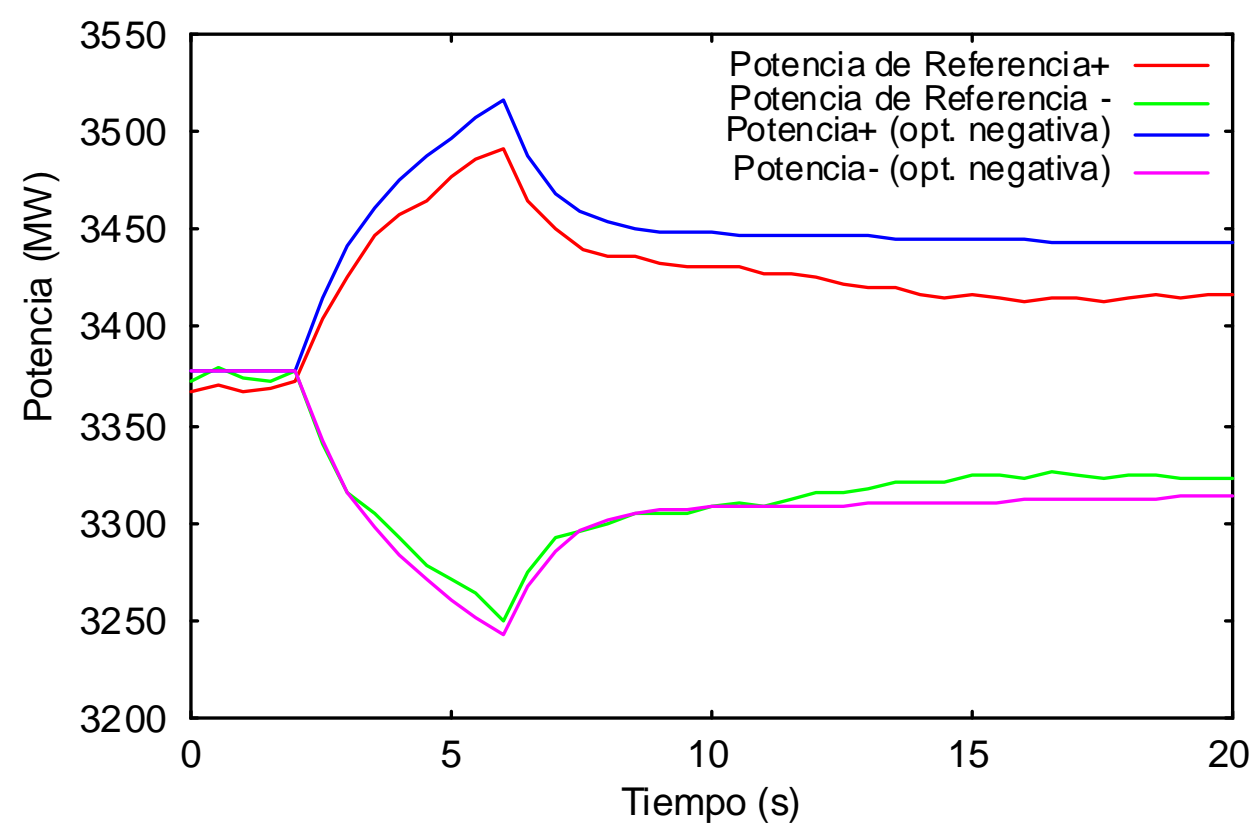

Figura 6.12: Comparación de las evoluciones de la potencia para los dos transitorios con los parámetros óptimos de la inserción negativa.

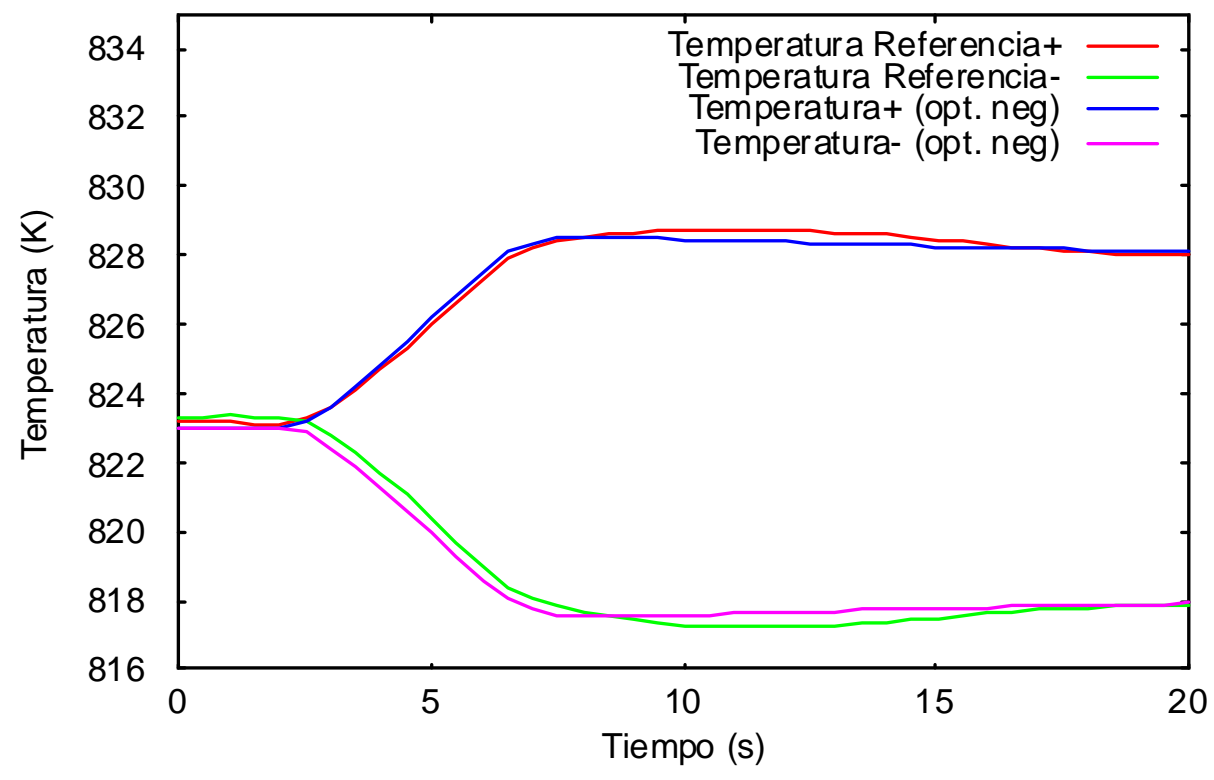

Figura 6.13: Comparación de las evoluciones de la temperatura del combustible para los dos transitorios con los parámetros óptimos de la inserción negativa.

De las gráficas anteriores se observa que los cálculos realizados con los parámetros obtenidos en la optimización de la inserción negativa de la reactividad también proporcionan unas evoluciones aceptables de la potencia y de la temperatura del 
combustible si se simula un transitorio de inserción positiva de reactividad. Los errores cometidos en cada uno de estos cálculos se muestran en la tabla 6.9.

\begin{tabular}{llcc}
\hline & Simulación & Error Potencia & Error Temperatura \\
\hline Parámetros óptimos & Inserción Positiva & 0.0059060 & 0.0002700 \\
\cline { 2 - 4 } Inserción positiva & Inserción Negativa & 0.0020156 & 0.0004216 \\
\hline Parámetros óptimos & Inserción Positiva & 0.0064508 & 0.0002308 \\
\cline { 2 - 4 } Inserción negativa & Inserción Negativa & 0.0024580 & 0.0003620 \\
\hline
\end{tabular}

Tabla 6.9: Errores obtenidos en los distintos cálculos considerados.

De los resultados expuestos en la tabla 6.9 se observa que el error conjunto cometido con respecto a la presión y a la temperatura es siempre mínimo para los valores óptimos que encuentra el MDS en cada transitorio.

A la vista de los resultados obtenidos en los diferentes ejemplos mostrados en este capítulo se observa la capacidad de la metodología de búsqueda y optimización de parámetros para obtener modelos reducidos de planta que presentan un buen comportamiento global para simular algunos transitorios de operación normal como son los transitorios de inserción de reactividad. No obstante, para ajustar un modelo reducido al comportamiento de una planta determinada va a ser necesario elegir una serie de transitorios y un conjunto de parámetros a optimizar y de variables de referencia adecuados para tener en cuenta con la mayor precisión las distintas situaciones posibles. 
Capítulo 6 


\section{Capítulo 7}

\section{Conclusiones}

La comprobación del comportamiento de las plantas nucleares requiere en muchas ocasiones de la simulación de estas instalaciones mediante códigos termohidráulicos que proporcionan cálculos aproximados al comportamiento real. Estas simulaciones vienen afectadas por una serie de incertidumbres que necesariamente deben cuantificarse para poder concluir que los resultados obtenidos mediante la simulación son representativos de la evolución real de la planta ante un accidente determinado. En concreto, tal como se señala en el capítulo 1 de esta tesis, las incertidumbres en las simulaciones se pueden asociar dos tipos fundamentales de causas que inducen a la aparición de errores asociados con las fuentes anteriores: las limitaciones en los modelos utilizados, así como en los datos disponibles, y los efectos de usuario.

Con el fin de cuantificar y acotar las incertidumbres introducidas en las simulaciones se han desarrollado diferentes metodologías, una de las cuales se presenta con detalle en el capítulo 2. Sin embargo, en el proceso que se sigue al aplicar estas metodologías aparecen diversas etapas que necesitan del juicio de expertos $\mathrm{y}$, por tanto, las incertidumbres debidas a los efectos de usuario son importantes.

Con la finalidad de eliminar, o al menos mitigar, este tipo de incertidumbres en la presente tesis se ha planteado y verificado la aplicabilidad de una nueva metodología sistemática que realiza la búsqueda de los parámetros de la simulación de forma automática y que utiliza una técnica de optimización para hacer mínimo el error entre el cálculo proporcionado por la simulación y una determinada referencia. Esta nueva metodología se basa en encontrar una solución al problema de búsqueda y optimización 
de parámetros mediante el establecimiento de una función error que dependiendo de dichos parámetros cuantifique la diferencia entre los resultados proporcionados por el código y las referencias, la cual se debe utilizar como función objetivo para ser minimizada mediante una técnica de optimización que busque el valor de dichos parámetros que hacen que el error cometido sea mínimo. La filosofía de esta nueva metodología es diferente a la planteada con las metodologías existentes de cálculo de incertidumbres puesto que no sólo pretende cuantificar el error cometido con una cierta combinación de parámetros termohidráulicos, sino que busca los valores más adecuados de los mismos que garanticen que el error cometido es el mínimo posible. Esta metodología se presenta en el capítulo tercero de esta tesis, en donde se plantean dos métodos generales de optimización con filosofías diferentes que pueden ser utilizados para resolver el problema de búsqueda y optimización de parámetros en simulaciones termohidráulicas. El objetivo de esta tesis es demostrar la aplicabilidad de la metodología planteada en la búsqueda y optimización de parámetros que deben estimarse al realizar una simulación mediante códigos termohidráulicos bajo diferentes condiciones de simulación.

En principio, cualquier método de optimización es susceptible de ser aplicado al problema de búsqueda y optimización de parámetros termohidráulicos, aunque la elección del método de optimización va en función del problema particular que se quiere resolver. En el capítulo 3 se han presentado dos técnicas de optimización diferentes, una de ellas consiste en la optimización del error cometido utilizando un algoritmo genético de estado estacionario, que corresponde a las llamadas técnicas heurísticas de optimización, y la otra técnica corresponde a las técnicas de búsqueda directa, en concreto se detalla el funcionamiento del método de búsqueda multidireccional (MDS). Ambas técnicas han sido analizadas para elegir aquella que proporcione mejores resultados en el problema de búsqueda y optimización de parámetros de simulaciones termohidráulicas.

En el capítulo 4 se ha aplicado la metodología automática de búsqueda y optimización de parámetros a modelos termohidráulicos sencillos, para los que es posible plantear un determinado problema y calcular su solución analítica. En concreto se han estimado los parámetros de un modelo de convección y de otro modelo de convección-difusión. La metodología de búsqueda y optimización de parámetros se ha aplicado haciendo uso de las dos técnicas de optimización. De los resultados obtenidos en los diferentes análisis realizados se concluye que:

1. Los análisis ponen de manifiesto la necesidad de escoger unos parámetros adecuados en la numérica del código, puesto que el cálculo que utiliza pasos de integración espacial y/o temporal excesivamente grandes proporcionan resultados afectados de grandes errores, mientras que las discretizaciones con 
pasos excesivamente pequeños únicamente se consigue aumentar el tiempo de cálculo.

2. Cualquiera de las normas planteadas para la cuantificación del error cometido en la simulación proporciona valores adecuados del mismo que permiten su minimización.

3. Las dos técnicas de optimización planteadas son capaces de obtener valores adecuados para los parámetros de búsqueda, sin embargo la más eficiente para resolver el problema de búsqueda y optimización de parámetros termohidráulicos es el método de búsqueda multidireccional, puesto que el tiempo de cálculo invertido es mucho menor. Esto es debido a la forma que presenta la función error, la cual hace que el algoritmo genético presente problemas de convergencia prematura.

La metodología propuesta es capaz de resolver problemas que afecten a las simulaciones realizadas mediante un código termohidráulico de estimación óptima, tal como se ha demostrado en el capítulo 5 de la tesis, donde se ha abordado el problema de búsqueda de valores de condiciones iniciales y de condiciones de contorno para una simulación determinada, puesto que son parámetros que frecuentemente se encuentran indeterminados o desconocidos cuando se pretende diseñar un modelo de planta para realizar una simulación utilizando un código termohidráulico. En concreto, se han estudiado dos ejemplos que forman parte de las matrices de validación del código termohidráulico RELAP5/Mod3, como son el Edward's pipe y algunos de los experimentos de secado del núcleo realizados en la instalación experimental del Royal Institute of Technology (RIT) de Estocolmo. De los estudios realizados se concluye que:

1. En el caso del estudio del Edward's pipe se ha demostrado la robustez de la metodología de búsqueda y optimización de parámetros termohidráulicos planteada ante el ruido del que puede verse afectada la variable utilizada como referencia para realizar el cálculo del error, situación normal si se trata de datos experimentales, en la que los parámetros de búsqueda han sido condiciones iniciales del fluido.

2. En la aplicación de la metodología a la búsqueda de las variables de contorno de una nodalización para RELAP5/Mod3.2 de la instalación experimental RIT se han considerado dos experimentos. De los resultados obtenidos en las diferentes aplicaciones se tiene que:

a. Para ambos experimentos se ha demostrado que la metodología de búsqueda y optimización de parámetros proporciona unos valores para las condiciones de contorno con los que los cálculos del código se 
ajustan más a los datos experimentales que con los que se facilitan en el modelo inicial.

b. Normalmente, al ampliar el número de parámetros en la optimización mejora el resultado final proporcionado por el código. No obstante la elección de los parámetros a buscar es importante puesto que a medida que aumenta el número de parámetros de búsqueda también aumenta el número de evaluaciones de la función objetivo necesarias para obtener el óptimo, por ello es importante realizar previamente una selección mediante un análisis de sensibilidad de los parámetros del modelo.

c. Sin embargo, todavía existe una clara discrepancia entre los resultados experimentales y los calculados por el código, lo que indica que sería necesaria una revisión de los modelos y de las correlaciones que el RELAP5 utiliza en algunas de las etapas de esta simulación.

3. En ambos casos se ha demostrado la importancia de fijar adecuadamente las condiciones iniciales y de contorno del modelo de planta que se esté utilizando en la simulación mediante un código termohidráulico de estimación óptima.

4. La metodología de búsqueda y optimización de parámetros es capaz de encontrar la mejor solución posible en cuanto al establecimiento del modelo de la instalación de manera automática, lo que elimina, o al menos reduce, la introducción de los efectos de usuario que se introducen durante el proceso de diseño de la nodalización más adecuada.

5. La metodología planteada es general y puede ser aplicada a la búsqueda y optimización de los parámetros que estén accesibles desde el fichero de entrada de cualquier código termohidráulico, siempre que se tengan datos experimentales que se puedan utilizar como referencia.

Para el estudio cualitativo del comportamiento de una planta es conveniente, en ocasiones, utilizar un modelo reducido de reactor. Esto es debido a que las simulaciones mediante códigos termohidráulicos involucran un gran número de modelos, el tiempo invertido en realizar un cálculo es excesivamente grande y ésto hace que la utilización de estos modelos sea más incómoda y no permita ver de forma clara el comportamiento de las variables importantes. 
Tomando como referencia una nodalización de RELAP5 para una planta típica del tipo PWR, en el capítulo 6 se ha desarrollado un modelo reducido de reactor sobre el que se ha aplicado la metodología de búsqueda y optimización de parámetros obteniendo las siguientes conclusiones:

1. El modelo reducido de reactor PWR de dos lazos asimétricos desarrollado en esta tesis, simula adecuadamente el comportamiento de la planta de referencia al que se ha ajustado y, por tanto, puede utilizarse como una buena aproximación al comportamiento de la planta completa para el estudio de ciertos transitorios.

2. El tiempo de cálculo que necesita el modelo reducido para calcular la evolución de las variables es mucho menor que el invertido por el RELAP5. Esta característica lo hace especialmente indicado para su uso en la aplicación de la metodología de búsqueda y optimización de parámetros.

3. La metodología de búsqueda y optimización de parámetros ha sido aplicada al modelo reducido de PWR para la determinación de algunos de los parámetros del modelo ante transitorios sencillos de operación, obteniendo unas evoluciones de las variables muy ajustados a las de referencia.

4. Los valores óptimos de los parámetros pueden cambiar para transitorios diferentes, sin embargo en casos similares los resultados obtenidos con los mismos valores son aceptables. De todas formas sería necesario la realización de una serie de transitorios para escoger los valores de los parámetros más adecuados con los que el modelo reducido fuese capaz de reproducir el comportamiento de la planta ante el mayor número de situaciones posibles.

5. Se ha comprobado la aplicabilidad de la metodología de búsqueda y optimización de parámetros considerando varias referencias asociadas a distintas variables del modelo. En este caso, la metodología sigue siendo válida si se implementa una función error que considere todas las referencias. Es más, la utilización del mayor número posible de dichas referencias es altamente recomendable. 
Ante todos estos resultados se plantean nuevas áreas abiertas al estudio siguiendo la línea planteada por la metodología de búsqueda y optimización de parámetros, tales como la realización de una optimización multiobjetivo con la finalidad de obtener los mejores valores para los parámetros termohidráulicos para una serie de funciones objetivo que se deseen optimizar.

Otra de las líneas futuras de trabajo que quedan planteadas en esta tesis es la reducción de los tiempos invertidos en las optimizaciones. Con este objeto sería conveniente la utilización de versiones paralelas del método MDS. Este método ha dado buenos resultados en su forma secuencial en cuanto a los valores de los parámetros y el tiempo invertido en la optimización; no obstante, debido a que presenta unas características aptas para la paralelización es conveniente aprovecharlas, lo que permitiría abordar problemas más complejos, como por ejemplo la consideración de un mayor número de parámetros a optimizar, la realización de transitorios que necesiten un tiempo de ejecución mayor, etc. Esta situación sería conveniente sobre todo para abordar problemas en los que se haga uso de un código termohidráulico detallado, como los presentados en el capítulo 5 de esta tesis, en los que se ha utilizado el código RELAP5, y en el que se ha señalado la limitación para abordar problemas más complejos debido al tiempo de cálculo necesario. 


\section{Capítulo 8}

\section{Referencias}

Abou 2000

Abou Khachfé R., Bailleul J.L. and Jarny Y. "The Simultaneous Determination of Thermal Conductivity and Heat Capacity within an orthotropic medium by using conjugated gradient algorithm". 16Th IMACS World Congress on Scientific Computation, Applied Mathematics and Simulation. Lausanne Switzerland. August 2125,2000 .

\section{Abreu1999}

Abreu C.M., Schirru R., Senra A. "Basic investigations related to genetic algorithms in core designs". Annals of Nuclear Energy 26 173-193, 1999.

\section{Accorsi1999}

Accorsi R., Marseguerra M., Padovani E., Zio E. "Neural estimation of first order sensitivity coefficients: Application to the control of a simulated pressurized water reactor". Nuclear Science and Engineering. Vol 132, pp-326-336, 1999.

\section{Ambrosini 1990}

Ambrosini W., Bovalini R., D'Auria F. "Evaluation of accuracy of thermal-hydraulic calculations". Energia Nucleare. Vol 2 Mayo-Septiembre 1990.

\section{Bäck1996}

Bäck T. "Evolutionary algorithms in theory and practice. Evolution strategies, evolutionary programming, genetic algorithms". Oxford University Press, NY,1996. 
Beasely1993a

Beasely D., Bull D., Martin R. "An overview of genetic algorithms: part 1, fundamentals". University Computing, Vol. 15:2, pp 58-69, 1993.

Beasely1993b

Beasely D., Bull D., Martin R. "An overview of genetic algorithms: part 2, research topics". University Computing, Vol. 15:4, pp 170-181, 1993.

Boyack1990

Boyack B.E., Catton I., Duffey R.B., Griffith P., Kastma K.R., Lellouche G.S., Levy S., Rohatgi U.S., Wilson G.E., Wulf W., Zuber N. "Quantifying reactor safety margins. Part 1: An overview of the Code Scaling, Applicability, and Uncertainty evaluation methodology". Nuclear Science and Design Vol. 119, pp 1-15, 1990.

Brown1989a

Brown P.N., Byrne G.D., Hindmarsh A.C. "VODE a variable-coefficient ODE solver". SIAM J. Sci. Stat. Comput., Vol. 10, pp 1038-1051, 1989.

Brown1989b

Brown P.N., Hindmarsh A.C. "Reduced storage matrix methods in stiff ODE systems". J. Appl. Math. \& Comp. Vol. 31, pp 40-91, 1989.

Burden1993

Burden R.L., Faires J.D. "Análisis numérico. 2a $2^{\mathrm{a}}$ Edición". Grupo editorial Iberoamericana, 1993.

Carlos 1998

Carlos S., Martorell S., Serradell V., Verdú G., Ródenas J., Saura J., Villanueva J.F. "Incorporación de mejoras en el ejercicio de intercomparación ISP-38". XXIV Reunión anual de la SNE, Valladolid, octubre 1998.

Carlos1999

Carlos S., Martorell S., Serradell V., Verdú G., Ródenas J. "Estudios de escenarios de parada con RELAP5/Mod3.2 y RELAP5/Mod3.2fg". II Jornada Técnica CAMP España. 1999.

Carlson1995

Carlson S. "A general method for handling constraints in genetic algorithms". University of Virginia, 1995. 
Catton1990

Duffey R.B., Shaw R.A., Boyack B.E., Griffith P., Kastma K.R., Lellouche G.S., Levy S., Rohatgi U.S., Wilson G.E.,Wulf W., Zuber N. "Quantifying reactor safety margins. Part 6: A physically based method of estimating PWR large break loss of coolant". Nuclear Science and Design, Vol. 119, pp 109-117, 1990 .

Cohen1995

Cohen S.D., Hindmarsh A.C. "Cvode, a stiff/nonstiff ode solver in C++". Proceedings of SciCADE95: Scientific Computing and Differential Equations. Standford University, 1995.

D'Auria1994

D'Auria F., Leonardi M., Pochard R. "Methodology for the evaluation of thermalhydraulic codes accuracy". Proc. of the International conference on New trends in Nuclear system thermohydraulics. Vol. 1. 30 Mayo- 2 Junio, Pisa, Italia, 1994.

D'Auria1995

D'Auria F., Debrecin N., Galassi G. "Outline of the uncertainty methodology on accuracy extrapolation". Nuclear Technology, Vol.109, pp 1-18, January 1995.

D'Auria2000

D'Auria F. and Gianotti W. "Development of a code with the capability of internal assessment of uncertainty". Nuclear Technology, Vol. 131, pp 159-196, August 2000.

Díaz1996

Díaz A., Glover F., Ghaziri H.M., Gonzalez J.L., Laguna M., Moscato P., Tseng S., "Optimización heurística y redes neuronales. En dirección de operaciones e ingeniería". Paraninfo, 1996.

Fletcher1991

Fletcher C.A.J. "Computational techniques for fluid dynamics. Volume 1". Springer, 1991.

Fogel1995

Fogel D.B. "Evolutionary conmputation". IEEE Press, NY,1995.

François 1999

François J.L., López H.A. "SOPRAG: a system for boiling water reactors reload pattern optimization using genetic algorithms". Annals of Nuclear Energy, Vol. 26, pp 1053 1063, 1999. 
Goldberg1989

Goldberg D.E. "Genetic algorithms in search, optimization and machine learning". Addison-Weslwy Pub. Co., Reading, MA, 1989.

Hassan1994;

Hassan Y.A., Baneerjee S.S. "RELAP5/Mod3 simulation of the loss of the residual heat removal system during midloop operation experiment conducted at ROSA-IV large scale test facility". Nuclear Technology Vol. 108, pp 191-206, 1994.

Henry1982

Henry A.F. "Nuclear-reactor analysis". The MIT press. 1982.

Herrera1996

Herrera F., Verdegay J.L. " Genetic algorithms and soft computing". Physica-Verlag Heidelberg, 1996.

Holland1975

Holland J. "Adaptation in natural and artificial systems". Ann Arbor, University Michigan Press, 1975.

INEL1995a

Idaho National Engineering Laboratory. "RELAP5/MOD3 code manual Volume I: Code structure, system models and solution methods" NUREG/CR-5535,Vol I, June 1995.

INEL1995b

Idaho National Engineering Laboratory. "RELAP5/MOD3 code manual Volume II: User's guide and input requirements" NUREG/CR-5535,Vol II, June 1995.

INEL1995c

Idaho National Engineering Laboratory. "RELAP5/MOD3 code manual Volume IV: Models and correlations" NUREG/CR-5535,Vol IV, June 1995.

INEL1995d

Idaho National Engineering Laboratory. "RELAP5/MOD3 code manual Volume V: User's Gudelines" NUREG/CR-5535,Vol V, June 1995.

INEL1996

Idaho National Engineering Laboratory. "RELAP5/MOD3 code manual Volume VII: Summaries and reviwes of independent code assessment reports" NUREG/CR-5535,Vol IV, April 1996. 
Ionescu-Bujor2000a

Ionescu-Bujor M., Caccuci D.G. "Adjoint Sensitivity Analysis of RELAP5/MOS3.2 Two-fluid Termal-hydraulic Code System-I". Nuclear Science and Engineering. Vol 136, pp 59-84, 2000.

Ionescu-Bujor2000b

Ionescu-Bujor M., Caccuci D.G. "Adjoint Sensitivity Analysis of RELAP5/MOS3.2 Two-fluid Termal-hydraulic Code System-II". Nuclear Science and Engineering. Vol 136, pp 85-121, 2000.

Karve1997

Karve A.A., Uddin R., Dorning J.J. "Stability analysis of BWR nuclear-coupled thermal-hydraulics using a simple model". Nuclear Engineering and Design. Vol 177, pp 155-177, 1997.

Kelley1999

Kelley C.T. " Iterative Methods for Optimization". SIAM 1999.

Lenisc1996

Lenisc D., Elliot L. and Ingham D.B. "Identification of Thermal conductivity and Heat Capacity in Unsteady Nonlinear Heat Conduction Problems Using The Boundary Element Method". Journal of Computational Physics, Vol 126, pp 410-420, 1996.

March-Leuba1986

March-Leuba J., Cacuci D.G., Perez R.B. "Nonlinear dynamics and stability of boiling water reactors: Part I- Qualitative analysis". Nuclear Science and Engineering. Vol 93, pp 111-123 (1986).

Martorell2000a

Martorell S., Carlos S., Verdú G., Serradell V., Ródenas J., Miró R., Llopis C. "Simulation of LOCA 6" and LOCA2" transients in the RHR of a PWR under low power conditions using RELAP5/Mod3.2". NUREG/IA-0171. February 2000.

Martorell2000b

Martorell S., Carlos S., Verdú G., Serradell V., Ródenas J., Miró R., Llopis C. "Simulation of station black-out in a PWR under midloop conditions using RELAP5/Mod3.2". NUREG/IA-0173. February 2000.

Martorell2000c

Martorell S., Carlos S., Sánchez A., Serradell V. " Constrained optimization of test intervals using a steady-state genetic algorithm". Reliability Engineering and System Safety, Vol 67, pp 215-232, 2000. 
Martorell2000d

Martorell S., Sánchez A., Carlos S., Serradell V. "Using genetic algorithms in completion times and tests intervals optimization with risk and cost constraints" Proc. of ESREL'2000-SRA Conf., 14-17 Edimburg. Ed: Balkema, May 2000.

Michalewicz1996

Michalewicz Z. "Genetic algorithms + data structures = Evolution programs (3 Ed.)". Springer-Verlag, Berlin, 1996.

Miller1996

Miller B.L., Goldberg D.E. "Optimal sampling for genetic algorithms". Illigal report $\mathrm{n}^{\circ}$ 96005, 1996.

Nilsson1993

Nilsson L. "Assessment of RELAP5/MOD3 Aganist Twenty-Five Post-Dryout Experiments Performed at the Royal Institute of Technology. NUREG/IA-009, 1993.

Park1992

Park M.G., Cho N.Z. "Design of a nonlinear model-based controller with adaptative PI gains for robust control of a nuclear reactor". Progress in Nuclear Energy, Vol 127, No.1, pp 37-49, 1992.

Park1993

Park M.G., Cho N.Z. "Time-optimal control of nuclear reactor power with adaptative proportional-integral-feedforward gains". IEE Transactions on Nuclear Science, Vol 40 No.3, pp 266-270, 1993.

Press 1992

Press W.H., Teukolsky S.A., Vetterling W.T., Flannery B.P. "Numerical Recipes in C. The art of scientific computing. $2^{\text {nd }}$ Edition". Cambrige University Press, 1992.

Su2001

Su J., Cotta R.M. “ Improved lumped parameter formulation for simplified LWR thermohydraulic analysis". Annals of Nuclear Energy, Vol. 28, pp 1019-1031. 2001.

Thomas1999

Thomas J.R. Jr, Herr J.D., Wood D.S. "Noise analysis method for monitoring the moderator temperature coefficient of pressurized water reactors: I. Theory". Nuclear Science and Engineering. Vol 108, pp 331-340, 1991. 
Torczon1989

Torczon V. "Multi-Directional Search: A Direct Search Method Algorithm for Parallel Machines". Tesis doctoral, Rice University Houston, May 1989.

Torczon 1990

Torczon V. "On the convergence of the multidirectional search algorithm". SIAM Journal on Optimization, Vol 1, No 1, pp 123-145, 1990.

Torczon2000a

Torczon V. " Pattern search methods for bound constrained minimization". SIAM Journal on Optimization Vol 9 No 4 pp 917-941, 2000.

Torczon2000b

Torczon V. "Pattern search methods for linearly constrained minimization". SIAM Journal on Optimization Vol 10 No 3 pp 1082-1099, 1999.

Wall1999

Wall M. "Galib: A C++ library of genetic algorithm components". Massachusetts Institute of Technology, 1999.

William1986

William W.G. Yeh. "Review of Parameter Identification Procedures in Groundwater Hydrology: The Inverse Problem". Water Resources Research. Vol 22, No. 2, pp 96$108,1986$.

Wilson 1990

Wilson G.E., Boyack B.E., Catton I., Duffey R.B., Griffith P., Kastma K.R., Lellouche G.S., Levy S., Rohatgi U.S., Wulf W., Zuber N. "Quantifying reactor safety margins. Part 2: Chacarterization of important contributos to uncertainty". Nuclear Science and Design, Vol 119, pp 17-31, 1990.

Wulf 1990

Wulf W., Boyack B.E., Catton I., Duffey R.B., Griffith P., Kastma K.R., Lellouche G.S., Levy S., Rohatgi U.S., Wilson G.E., Zuber N. "Quantifying reactor safety margins. Part 3: Assessment and ranging of parameters". Nuclear Science and Design, Vol. 119, pp 33-65, 1990.

Zuber 1990

Zuber N., Wilson G.E., Boyack B.E., Catton I., Duffey R.B., Griffith P., Kastma K.R., Lellouche G.S., Levy S., Rohatgi U.S., Wulf W. "Quantifying reactor safety margins. Part 5: Evaluation of scale-up capabilities". Nuclear Science and Design 119, 97-107, 1990. 
Capítulo 8 
Apéndice 1

Fichero de entrada del Edward's pipe para RELAP5. 


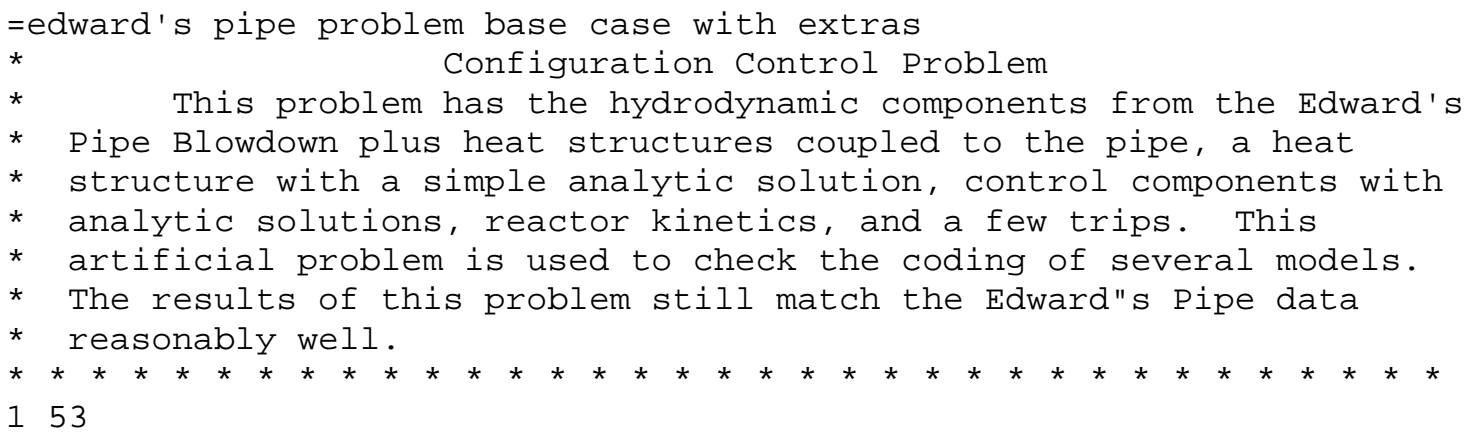




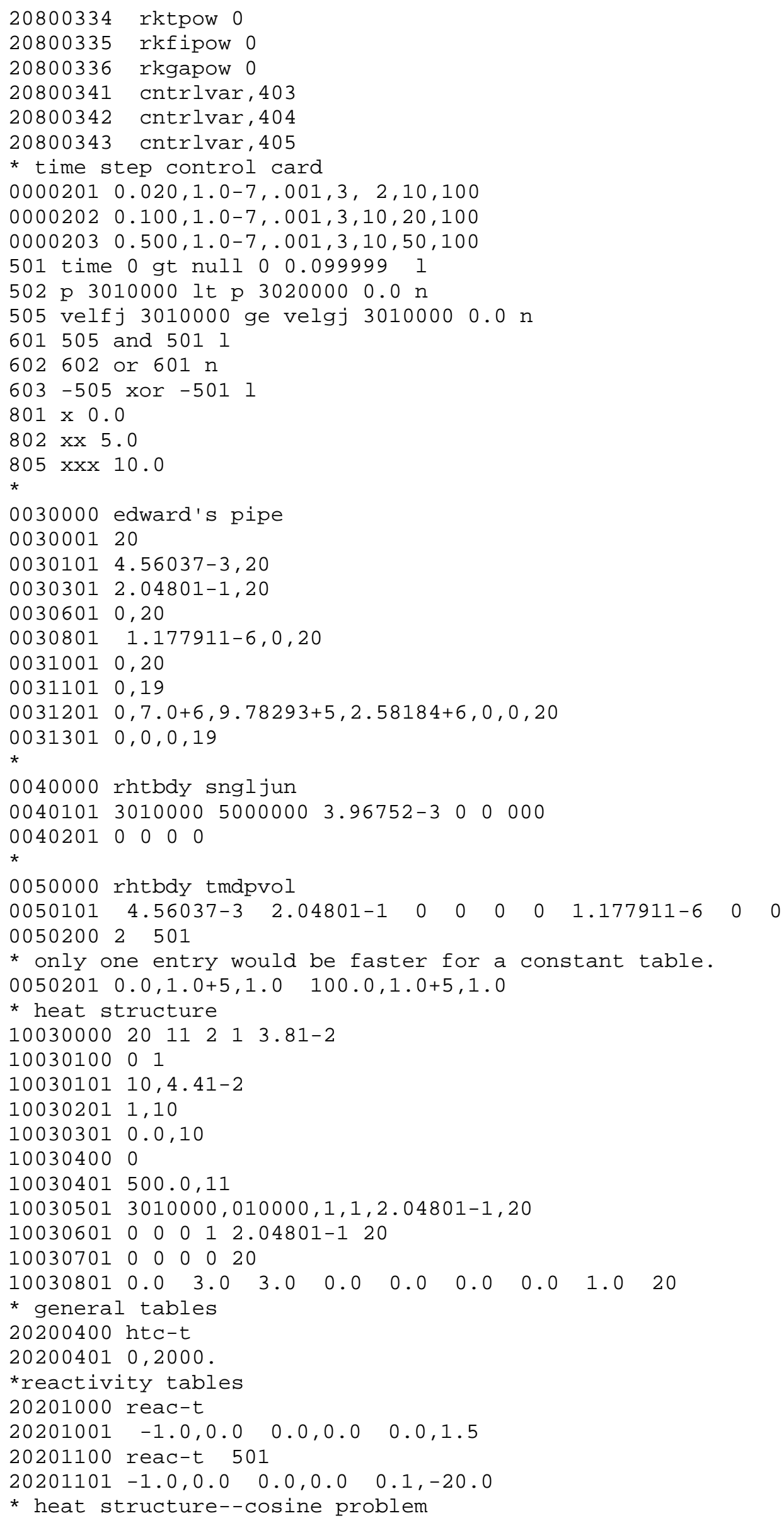




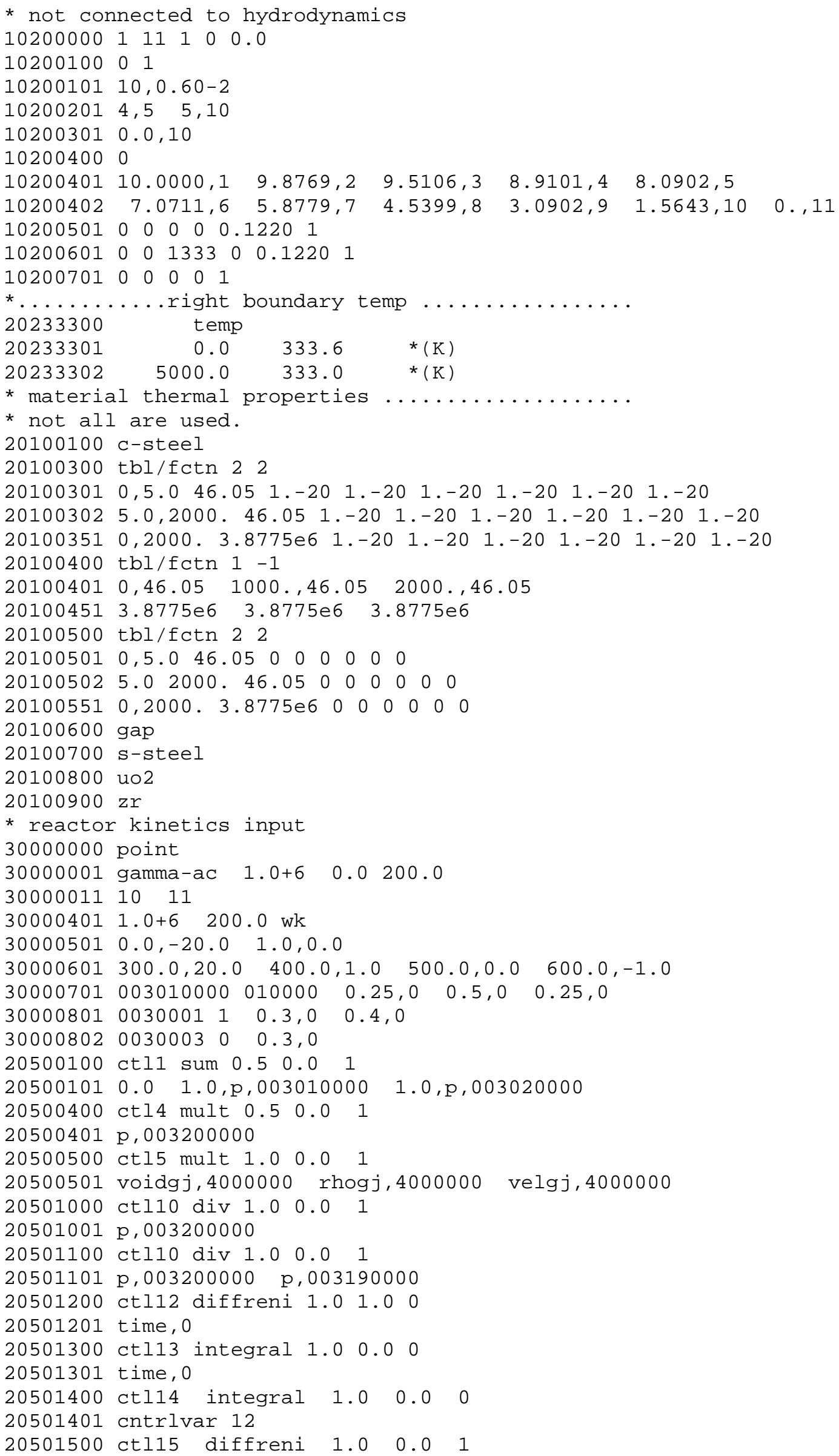




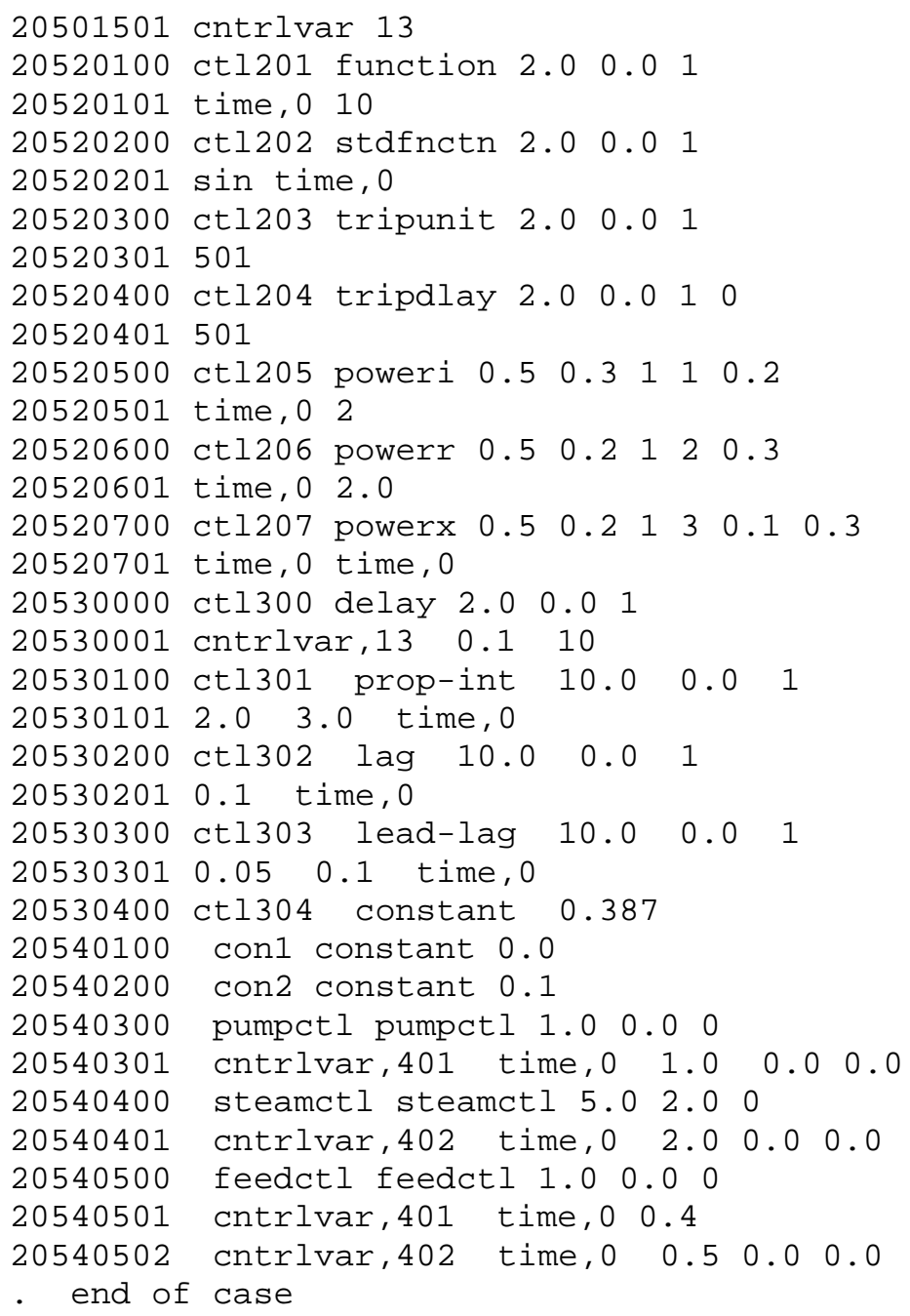


Fichero del Edwad's pipe 
Apéndice 2

Fichero de entrada del RIT 136 para RELAP5 


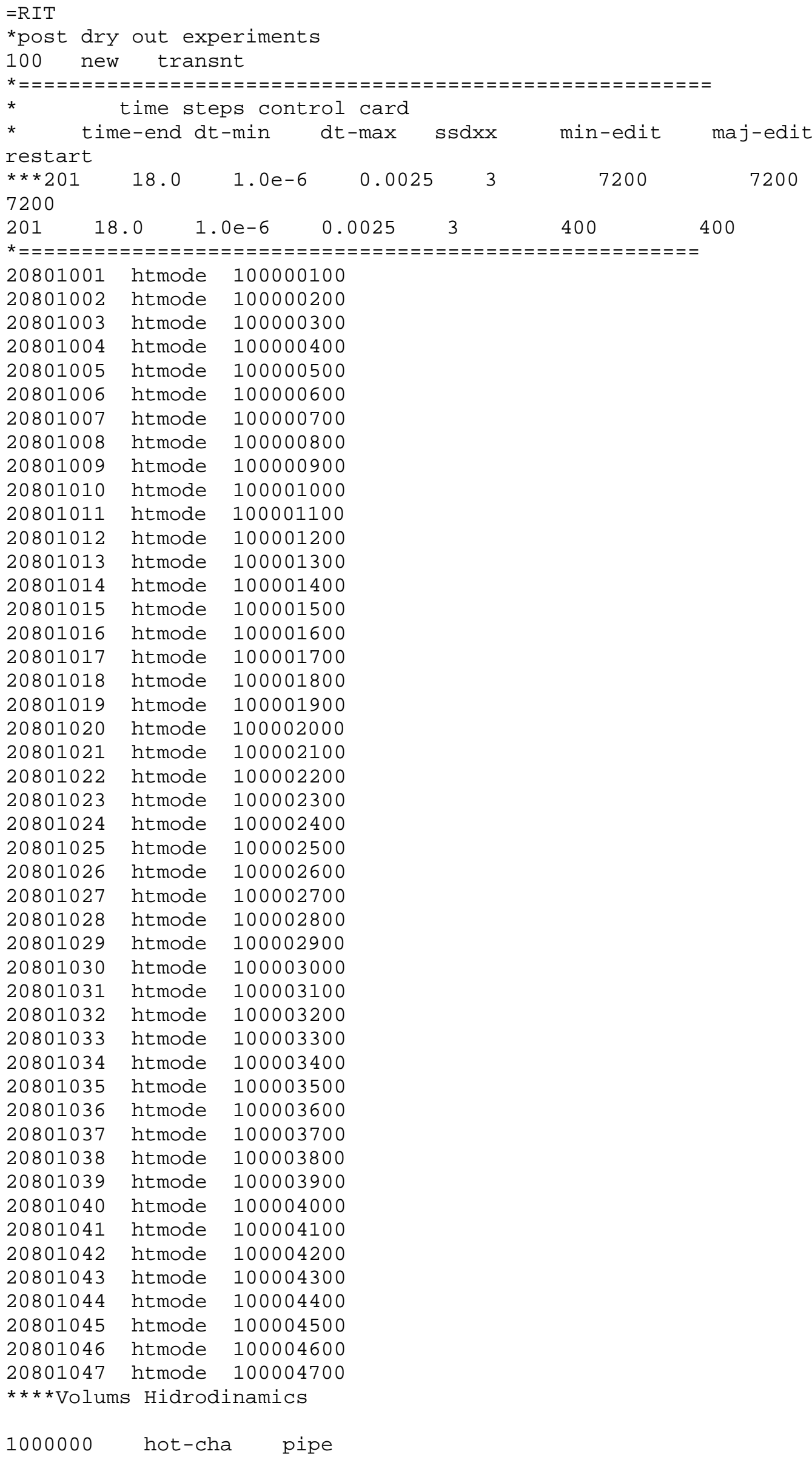




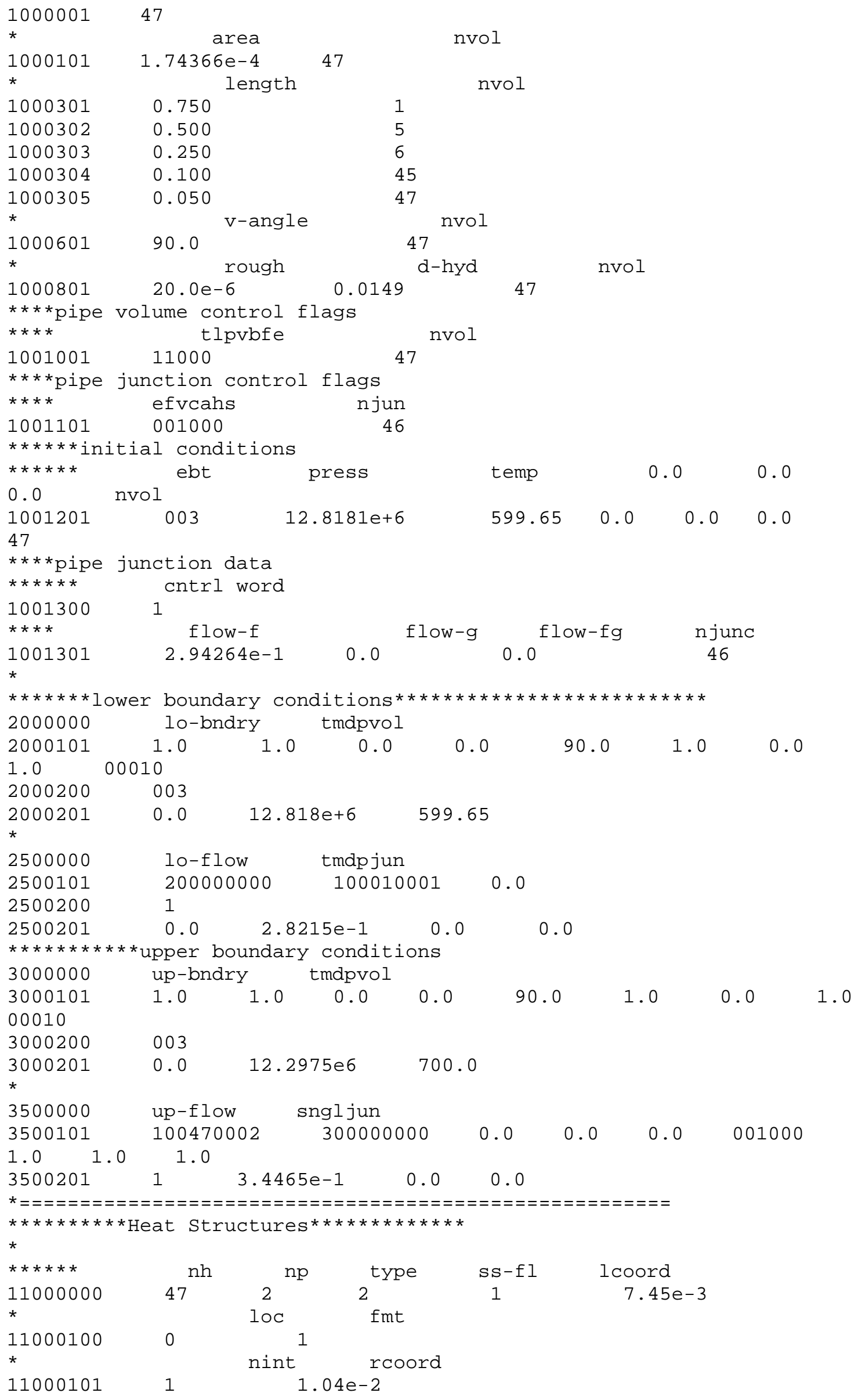




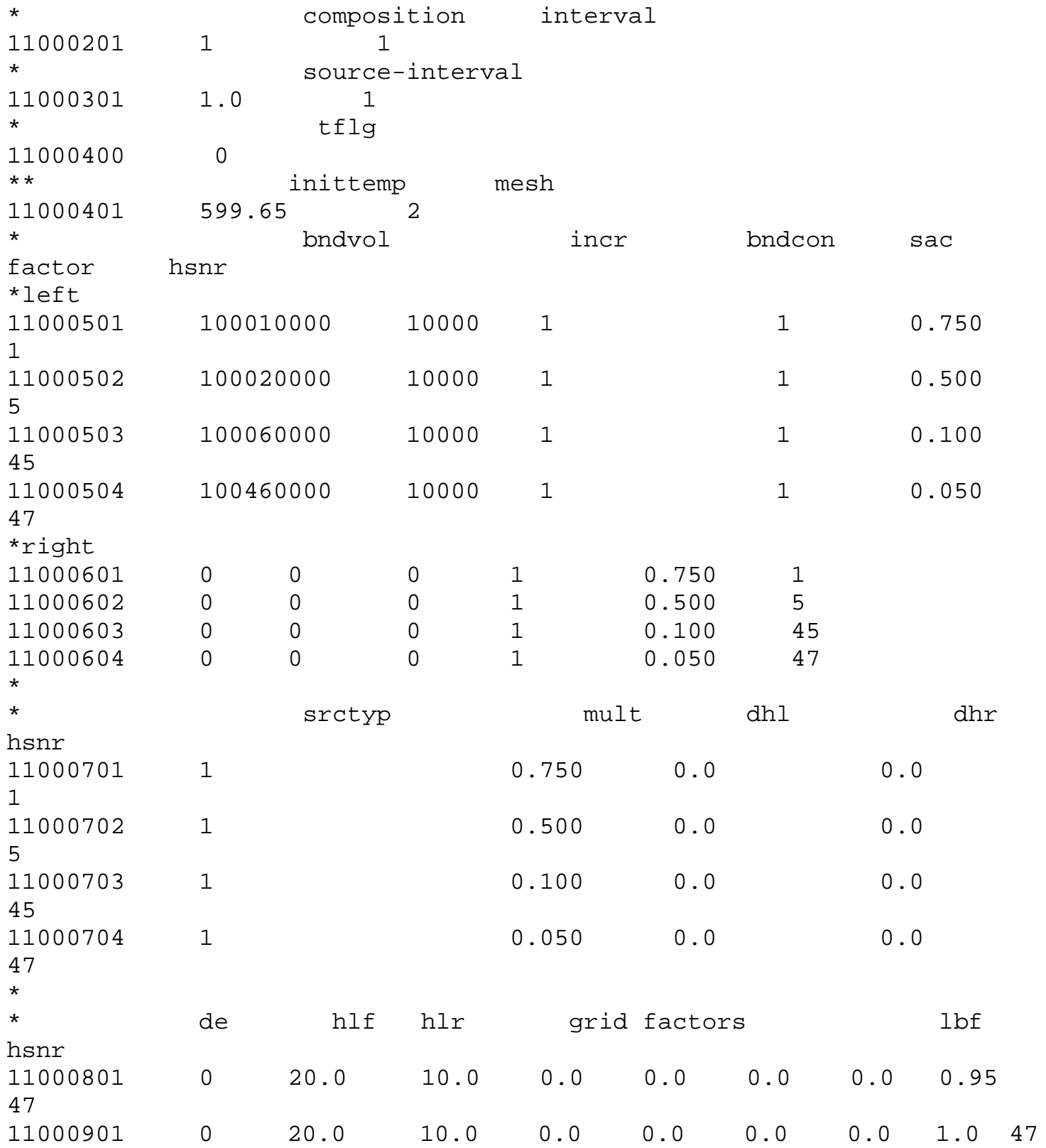


Fichero del RIT 
Apéndice 3

Fichero de entrada del PWR de dos lazos para RELAP5 
=typical pwr model -- 4 inch cold leg break 36.05 check case

* Configuration Control Problem

* This problem is a simulation of a four loop presurized reactor

* undergoing a small break. Loop containing break is modeled as a

* single loop but the other three loops are coalesced into one loop.

* Modeling does not now follow all recommended modeling practices but

* problem is still good test of many features of code. Problem uses

* standard matrix techniques.

$* * * * * * * * * * * * * * * * * * * * * * * * * * * * * * * * * * *$

100 new transnt

*

102 british si

1055.06 .05000 .0

110 nitrogen

*****************************************************************

$*$

$*$

****************************************************************

$*$

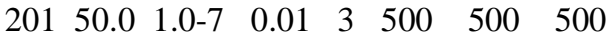

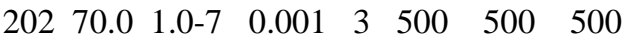

*

20800004 errmax 0

20800005 systms 1

20800006 systms 2

20800007 systms 3

20800008 rkreac 0

20800009 rktpow 0

**************************************************************

$*$

* minor edit variables

$* * * * * * * * * * * * * * * * * * * * * * * * * * * * * * * * * * * * * * * * * * * * * * * * * * * * * * * * * *$

$301 \mathrm{p} \quad 345010000$

302 p 180010000

$303 \mathrm{p} \quad 280010000$

304 voidg 345010000

305 voidg 335060000

306 voidg 212010000

351 mflowj 505000000

***************************************************************************

trip cards

$*$

trip idenifier

$*$

501 scram signal,rc pump trip,steam generator main feed and main steam outlet trip

502 initiate power decay curve

503 pressure trip for si and charging initiation *

504 si and charging initiation with 5.0 sec delay *

* 505 aux feed flow initiation with $14.0 \mathrm{sec}$ delay *

* 506 break initiation

* 507 time zero trip

* $508\langle * * * * * * * * * * * * * * * * *>$

* $509<$ porv trip logic >

* $601<$

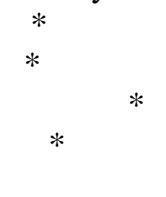




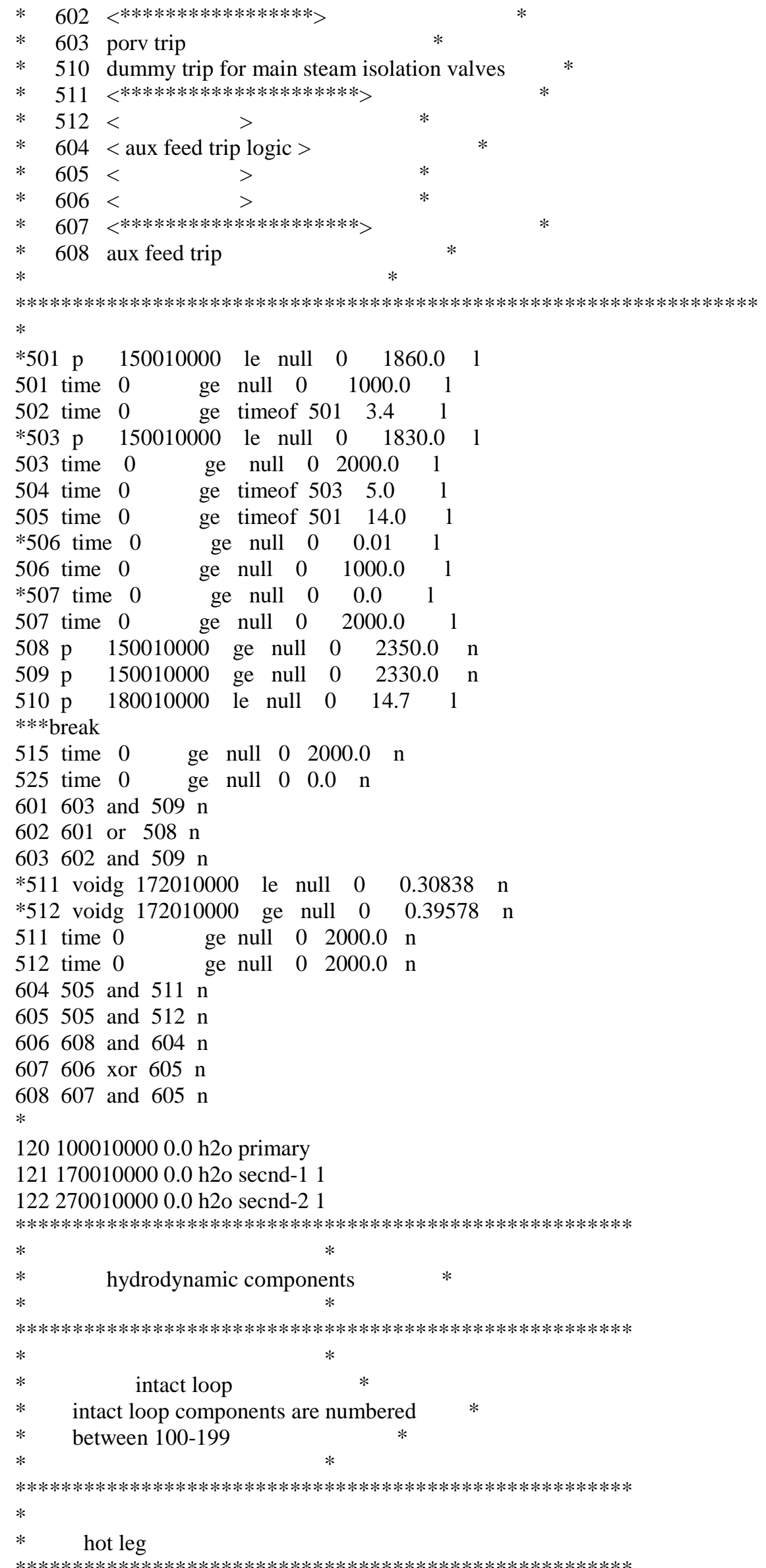




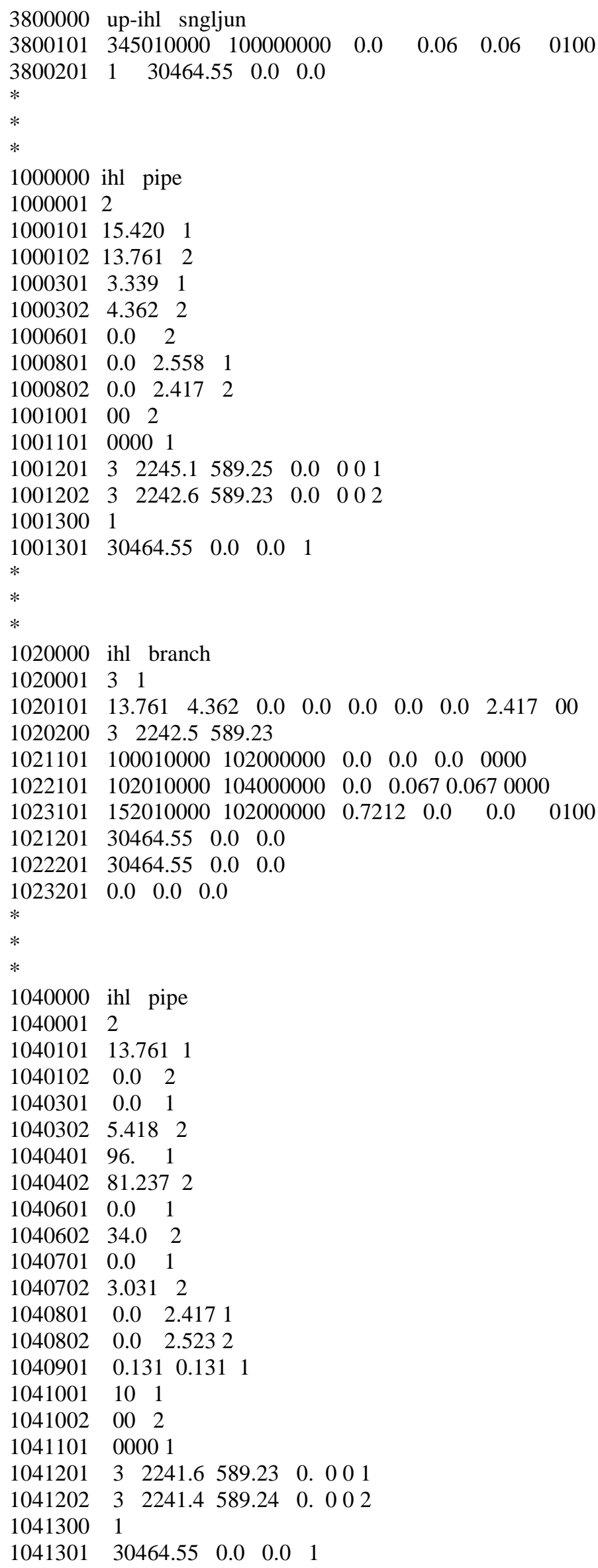




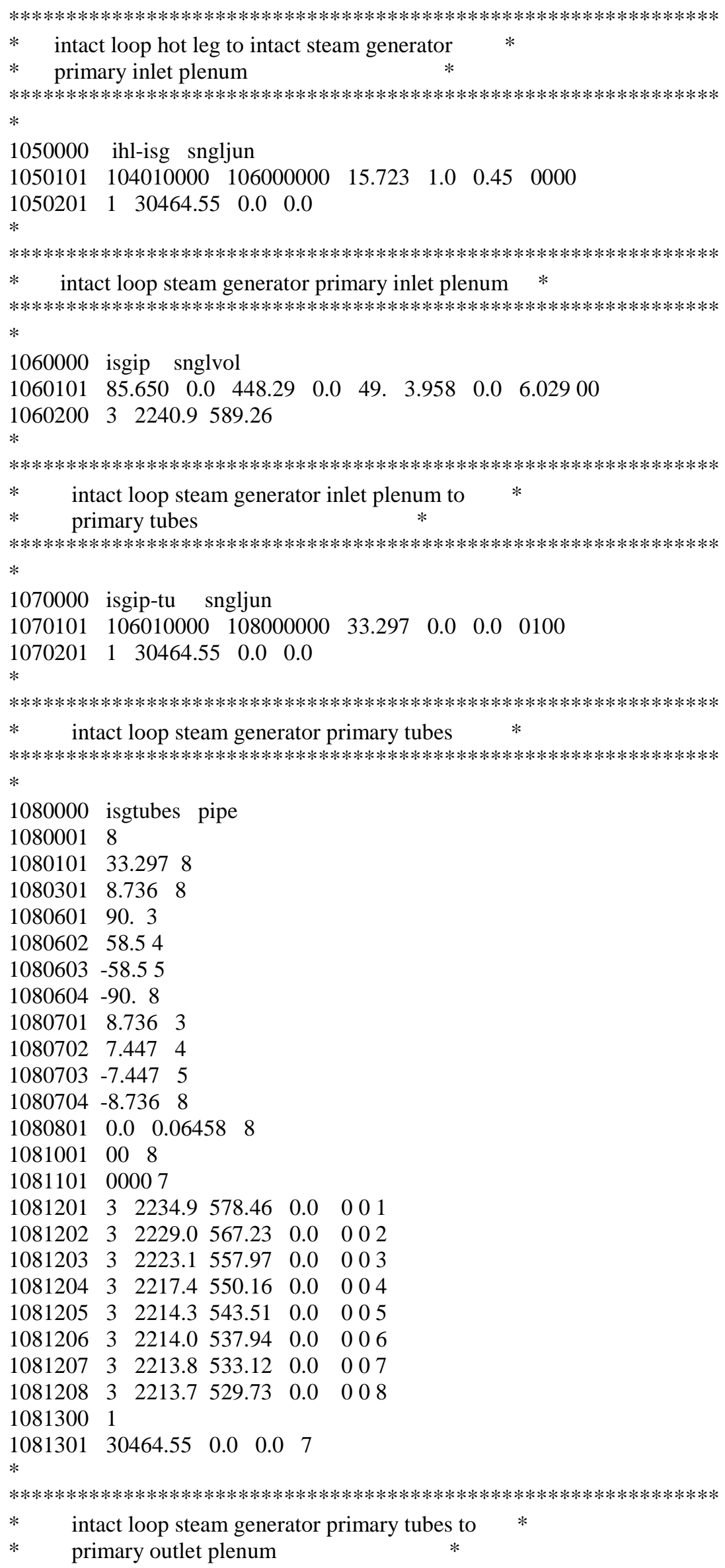




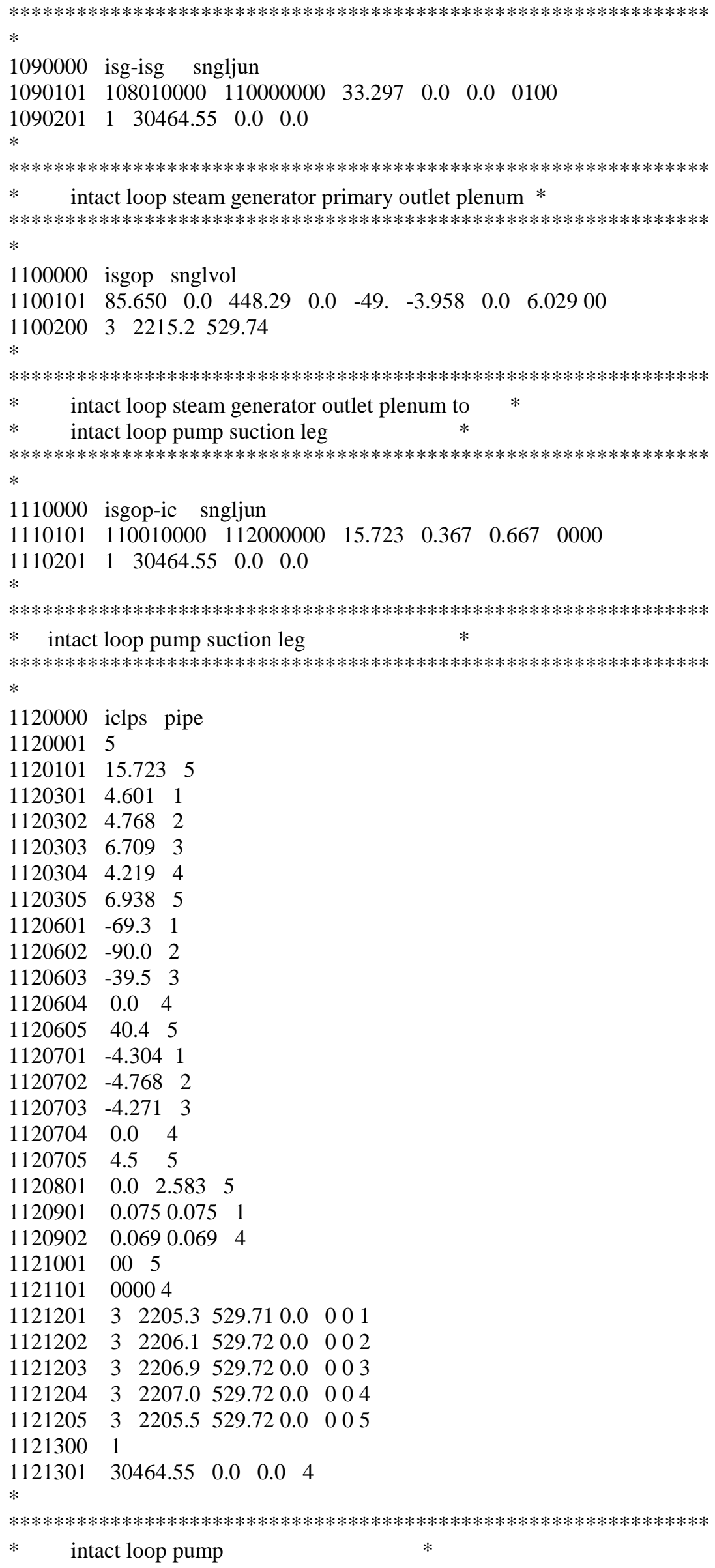




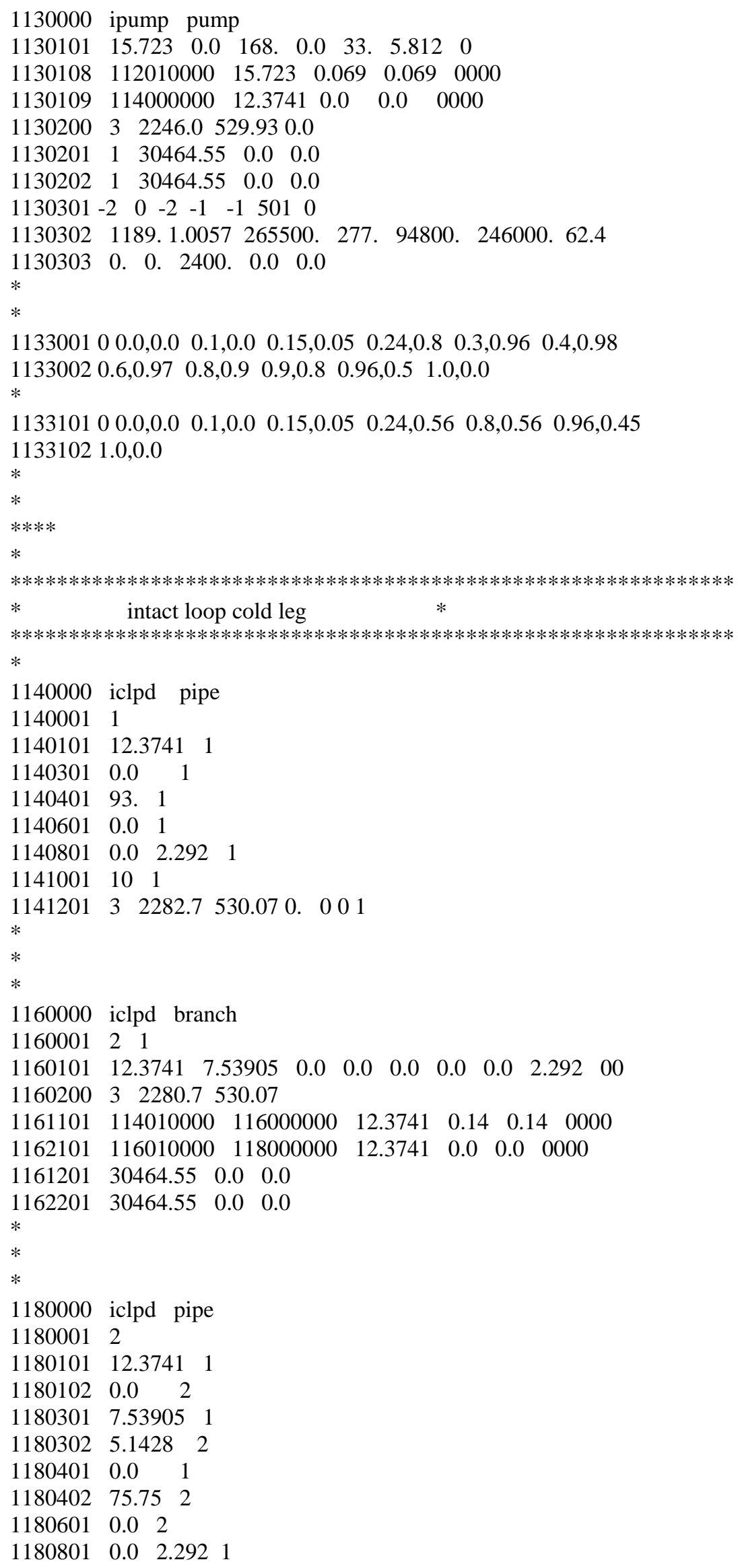




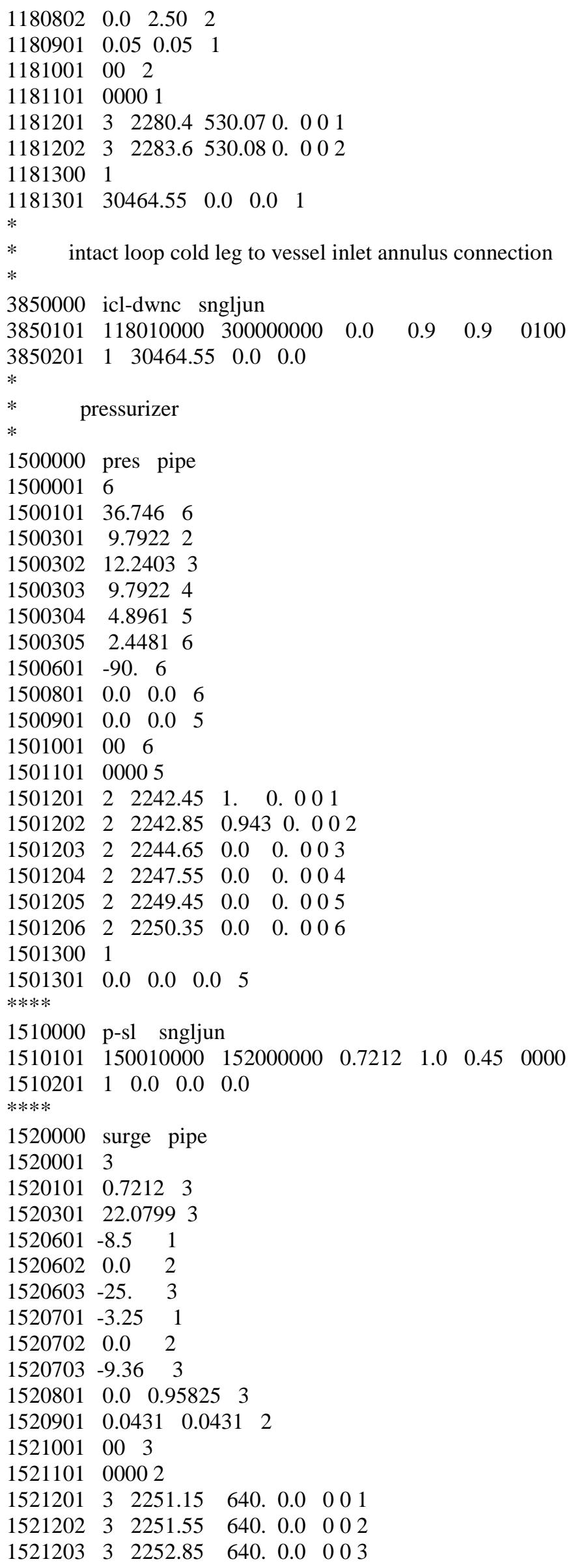




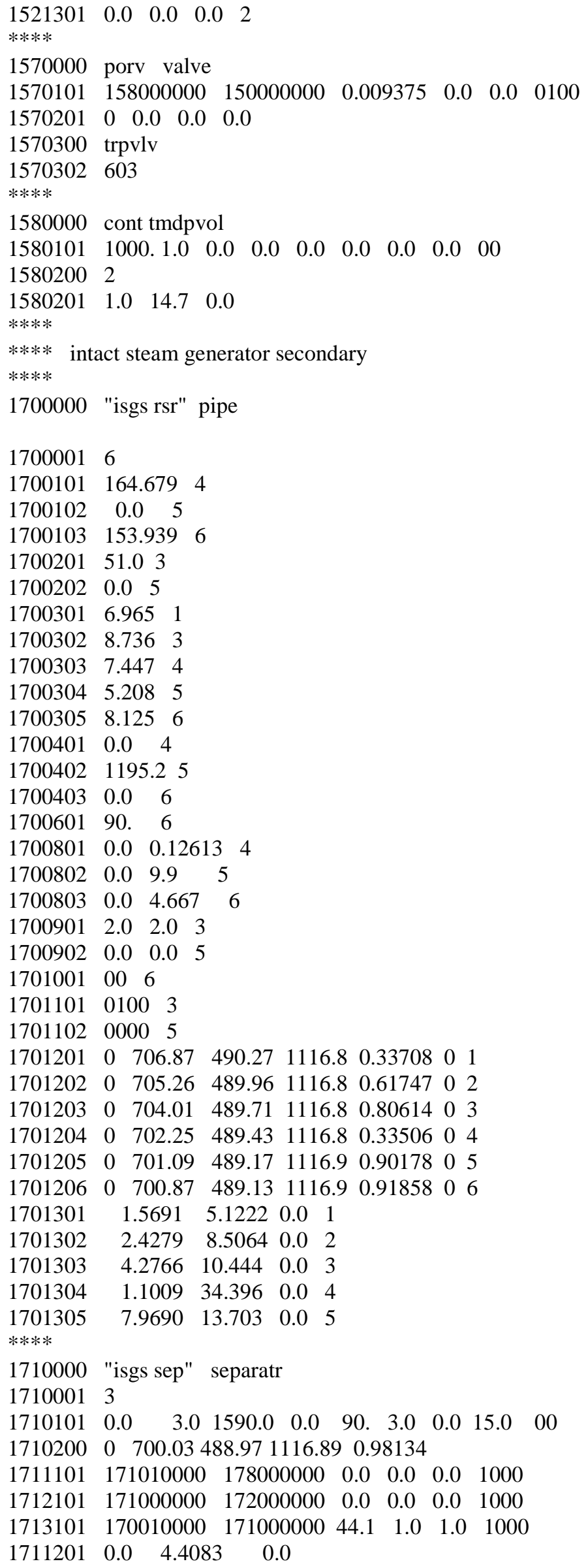




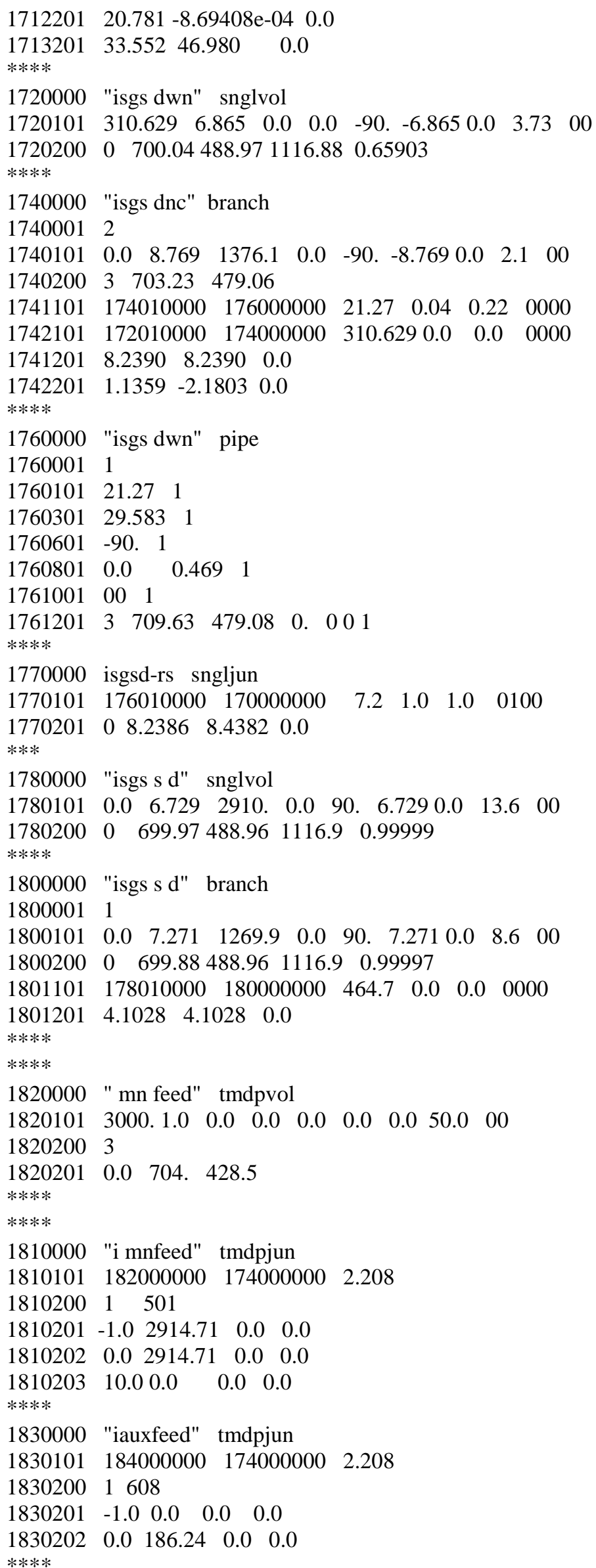




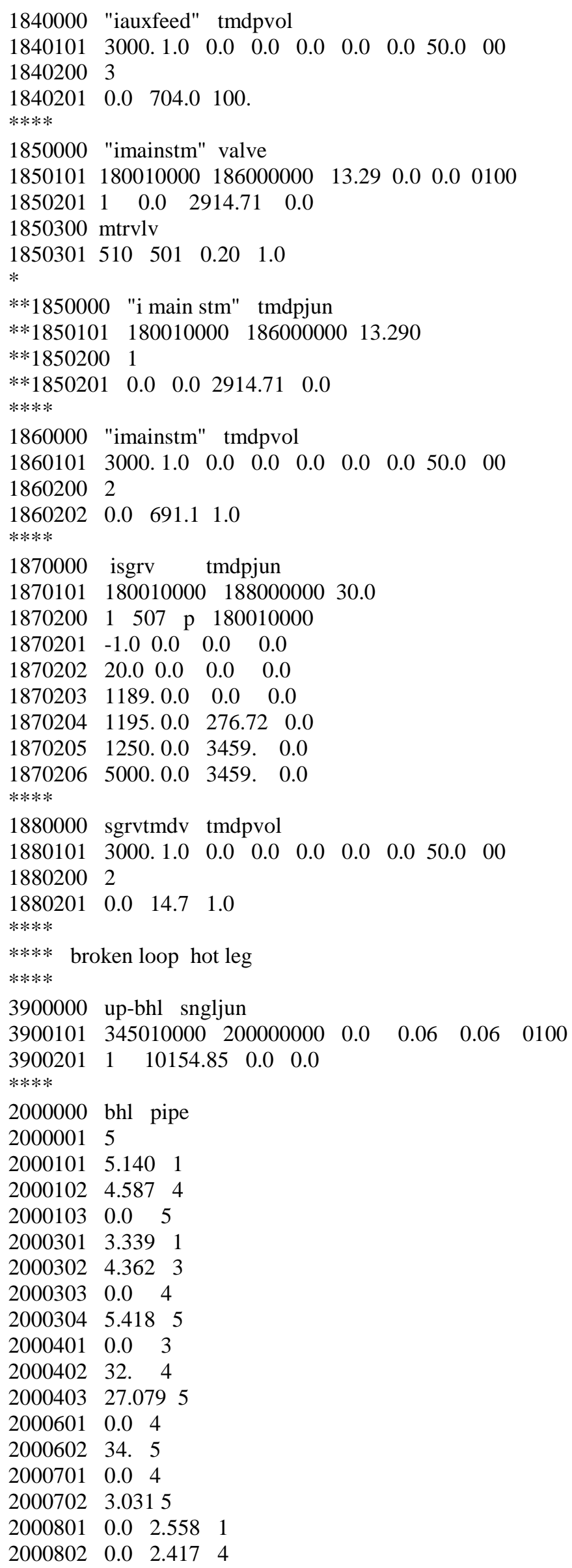




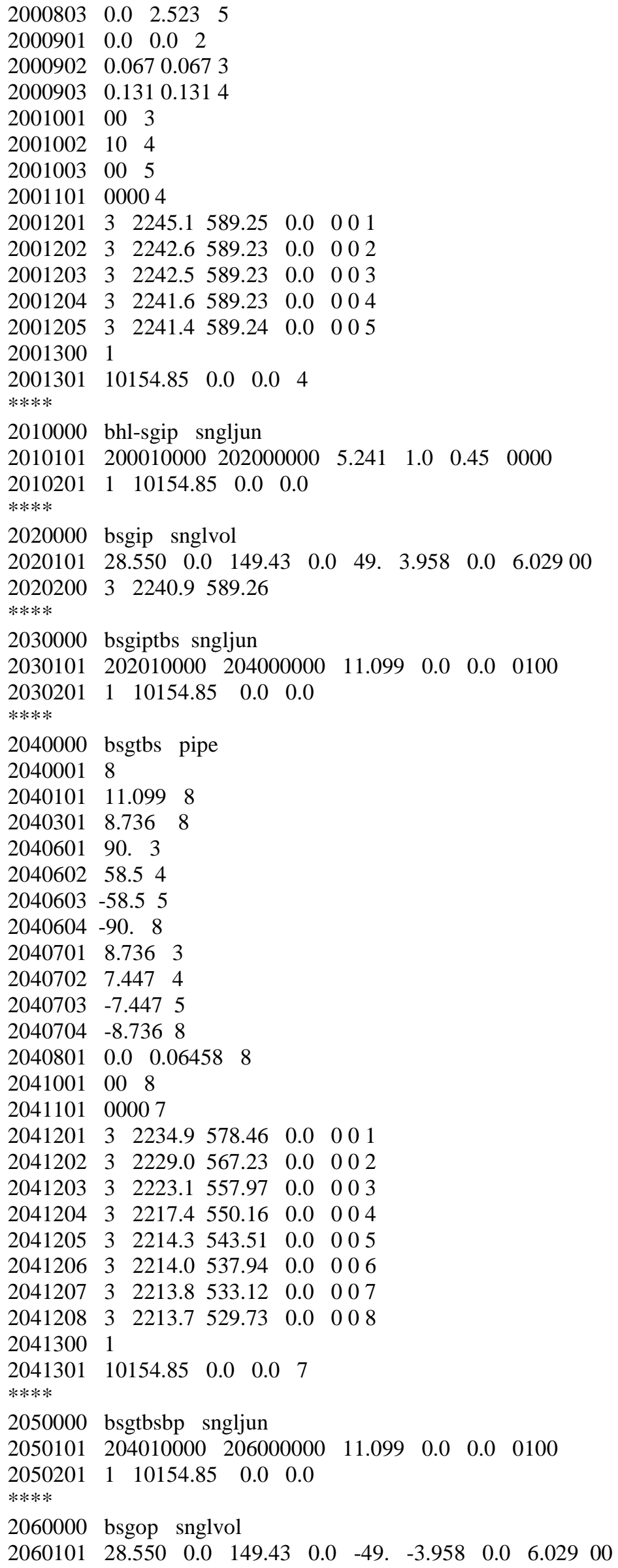




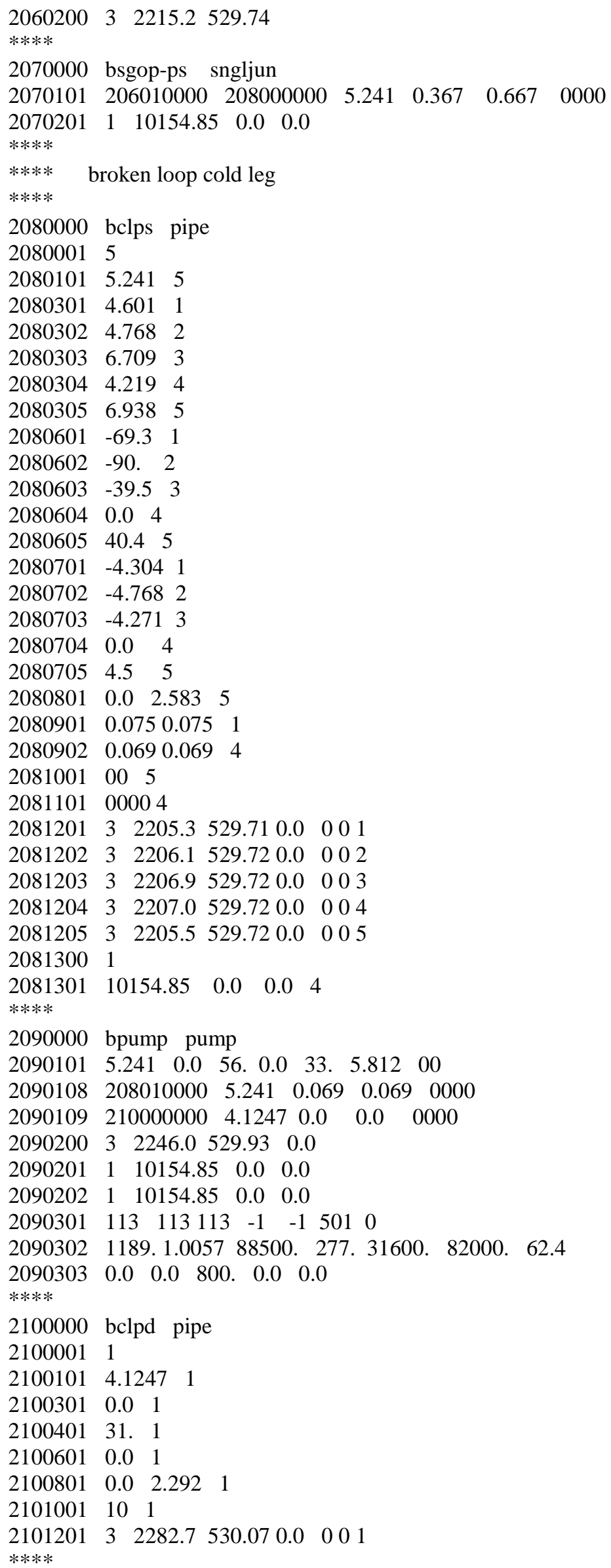




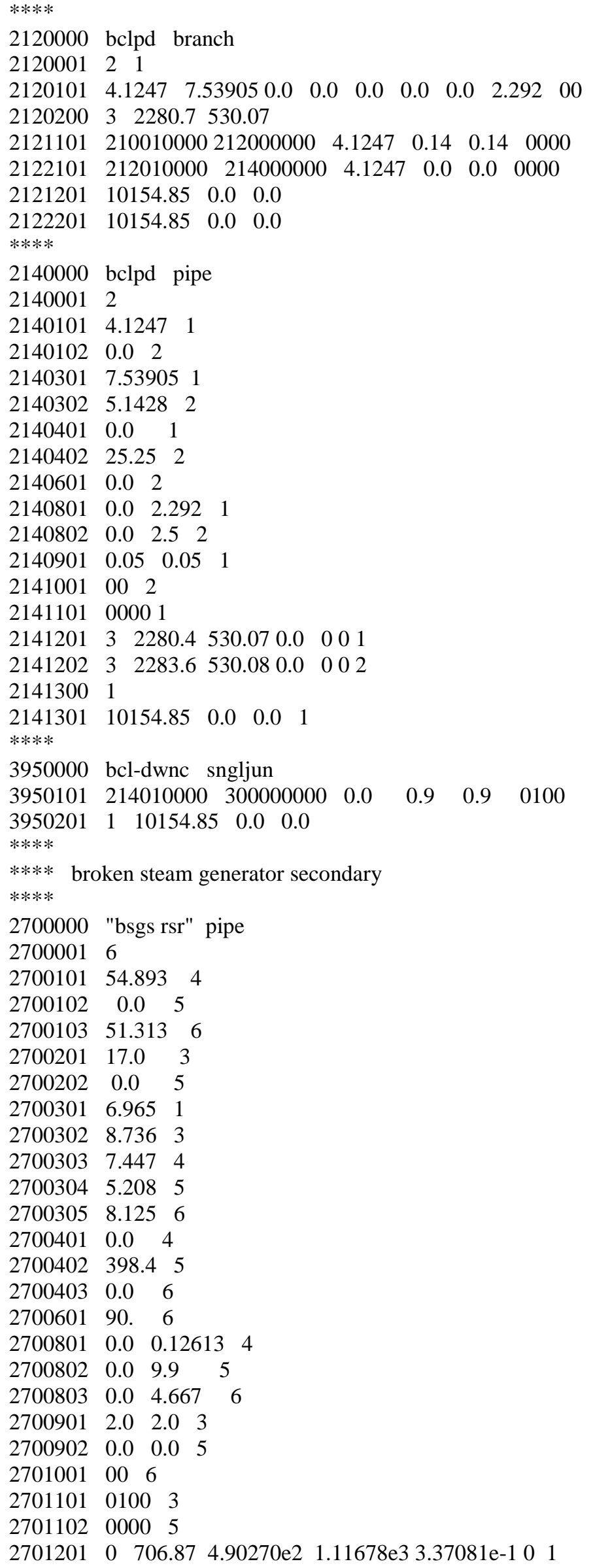


$\begin{array}{llllllll}2701202 & 0 & 705.26 & 4.89959 \mathrm{e} 2 & 1.11680 \mathrm{e} 3 & 6.17471 \mathrm{e}-1 & 0 & 2\end{array}$ $\begin{array}{lllllllll}2701203 & 0 & 704.01 & 4.89713 \mathrm{e} 2 & 1.11682 \mathrm{e} 3 & 8.06138 \mathrm{e}-1 & 0 & 3\end{array}$ $\begin{array}{lllllllll}2701204 & 0 & 702.25 & 4.89428 \mathrm{e} 2 & 1.11685 \mathrm{e} 3 & 3.35057 \mathrm{e}-1 & 0 & 4\end{array}$ $\begin{array}{lllllllll}2701205 & 0 & 701.09 & 4.89171 \mathrm{e} 2 & 1.11686 \mathrm{e} 3 & 9.01781 \mathrm{e}-1 & 0 & 5\end{array}$ $\begin{array}{lllllllll}2701206 & 0 & 700.87 & 4.89132 \mathrm{e} 2 & 1.11687 \mathrm{e} 3 & 9.18578 \mathrm{e}-1 & 0 & 6\end{array}$

$\begin{array}{lllll}2701301 & 1.5691 & 5.1222 & 0.0 & 1\end{array}$

$\begin{array}{llllll}2701302 & 2.4279 & 8.5064 & 0.0 & 2\end{array}$

$\begin{array}{lllll}2701303 & 4.2766 & 10.444 & 0.0 & 3\end{array}$

$\begin{array}{lllll}2701304 & 1.1009 & 34.396 & 0.0 & 4\end{array}$

$\begin{array}{lllll}2701305 & 7.9690 & 13.703 & 0.0 & 5\end{array}$

$* * * *$

2710000 "bsgs spr" separatr

27100013

$\begin{array}{llllllllll}2710101 & 0.0 & 3.0 & 530.0 & 0.0 & 90 . & 3.0 & 0.0 & 15.0 & 00\end{array}$

$\begin{array}{lllllll}2710200 & 0 & 700.03 & 4.88974 \mathrm{e}+2 & 1.11689 \mathrm{e} 3 & 9.843 \mathrm{e}-1\end{array}$

$\begin{array}{lllllll}2711101 & 271010000 & 278000000 & 0.0 & 0.0 & 0.0 & 1000\end{array}$

$\begin{array}{llllllll}2712101 & 271000000 & 272000000 & 0.0 & 0.0 & 0.0 & 1000\end{array}$

$\begin{array}{llllllll}2713101 & 270010000 & 271000000 & 14.7 & 1.0 & 1.0 & 1000\end{array}$

$\begin{array}{llll}2711201 & 0.0 & 4.4083 & 0.0\end{array}$

$2712201 \quad 20.781-8.69408 \mathrm{e}-04 \quad 0.0$

$\begin{array}{llll}2713201 & 33.552 & 46.980 & 0.0\end{array}$

****

2720000 "bsgs dwn" snglvol

$\begin{array}{llllllllll}2720101 & 103.543 & 6.865 & 0.0 & 0.0 & -90 & -6.865 & 0.0 & 3.73 & 00\end{array}$

$2720200 \quad 0 \quad 700.04 \quad 4.88972 \mathrm{e} 2 \quad 1.11688 \mathrm{e} 3 \quad 6.59034 \mathrm{e}-1$

****

2740000 "bsgs dnc" branch

27400012

$\begin{array}{llllllllll}2740101 & 0.0 & 8.769 & 458.7 & 0.0 & -90 & -8.769 & 0.0 & 2.1 & 00\end{array}$

$\begin{array}{llll}2740200 & 3 & 703.23 & 479.06\end{array}$

$\begin{array}{lllllll}2741101 & 274010000 & 276000000 & 7.09 & 0.04 & 0.22 & 0000\end{array}$

$\begin{array}{lllllll}2742101 & 272010000 & 274000000 & 103.543 & 0.0 & 0.0 & 0000\end{array}$

$\begin{array}{llll}2741201 & 8.2390 & 8.2390 & 0.0\end{array}$

$\begin{array}{llll}2742201 & 1.1359 & -2.1803 & 0.0\end{array}$

****

2760000 "bsgs dwn" pipe

27600011

$27601017.09 \quad 1$

$\begin{array}{lll}2760301 & 29.583 & 1\end{array}$

$2760601-90.1$

$\begin{array}{llll}2760801 & 0.0 & 0.469 & 1\end{array}$

$2761001 \quad 00 \quad 1$

$\begin{array}{lllllllll}2761201 & 3 & 709.63 & 479.08 & 0 . & 0 & 0 & 1\end{array}$

$* * * *$

2770000 bsgsdrsr sngljun

$\begin{array}{lllllll}2770101 & 276010000 & 270000000 & 2.4 & 1.0 & 1.0 & 0100\end{array}$

$\begin{array}{lllll}2770201 & 0 & 8.2386 & 8.4382 & 0.0\end{array}$

****

2780000 "bsgs s d" snglvol

$\begin{array}{llllllllll}2780101 & 0.0 & 6.729 & 970 & 0.0 & 90 & 6.729 & 0.0 & 13.6 & 00\end{array}$

$\begin{array}{llllll}2780200 & 0 & 699.97488 .96 & 1116.9 & 0.99999\end{array}$

****

2800000 "bsgs s d" branch

28000011

$\begin{array}{llllllllll}2800101 & 0.0 & 7.271 & 423.3 & 0.0 & 90 . & 7.271 & 0.0 & 8.6 & 00\end{array}$

$\begin{array}{llllll}2800200 & 0 & 699.88 & 4.88947 \mathrm{e} 2 & 1.11692 \mathrm{e} 3 & 9.99999 \mathrm{e}-1\end{array}$

$\begin{array}{llllllll}2801101 & 278010000 & 280000000 & 154.9 & 0.0 & 0.0 & 0000\end{array}$

$\begin{array}{lllll}2801201 & 4.1028 & 4.1028 & 0.0\end{array}$

$* * * *$

2810000 "b mnfeed" tmdpjun 


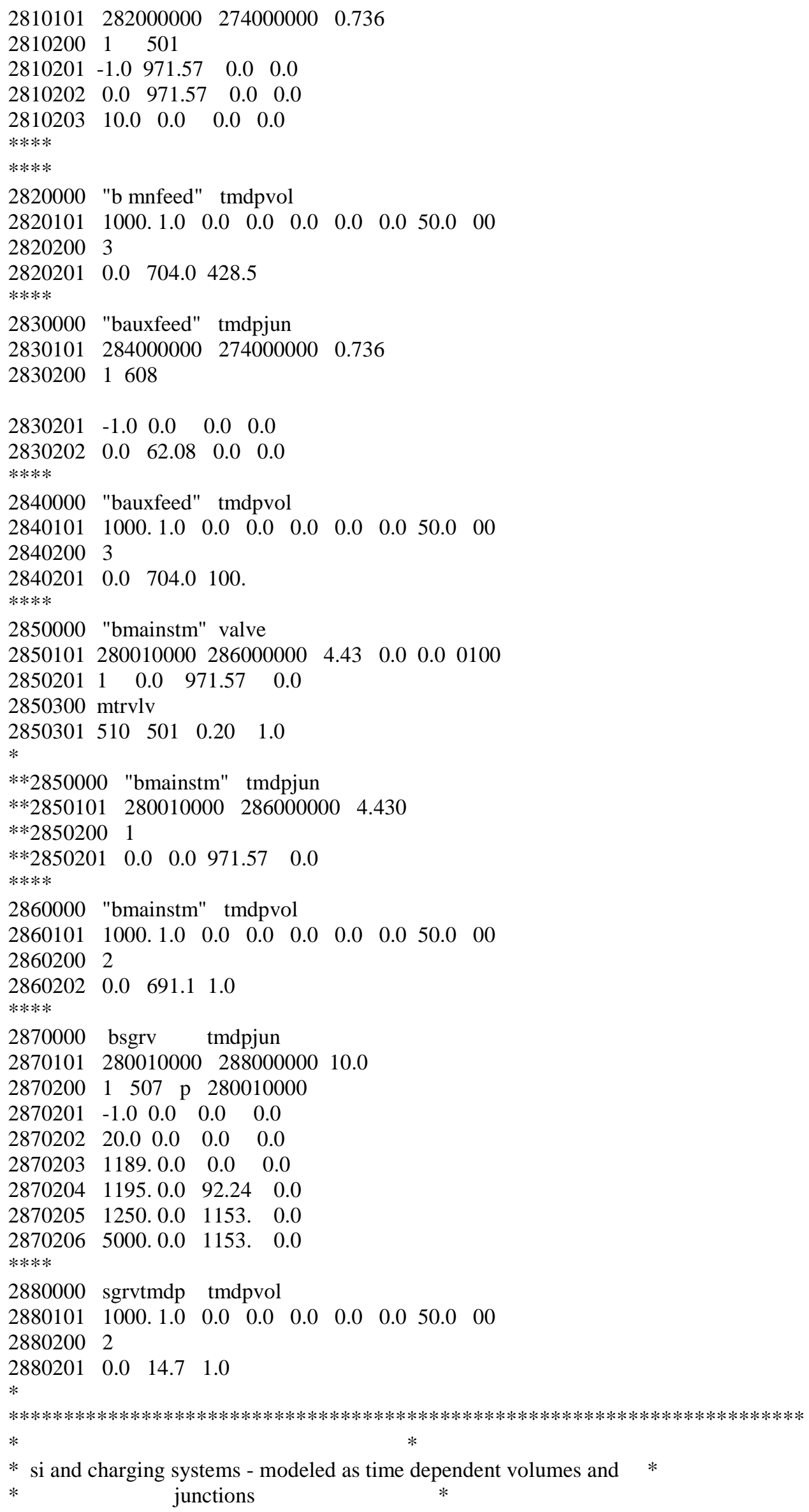




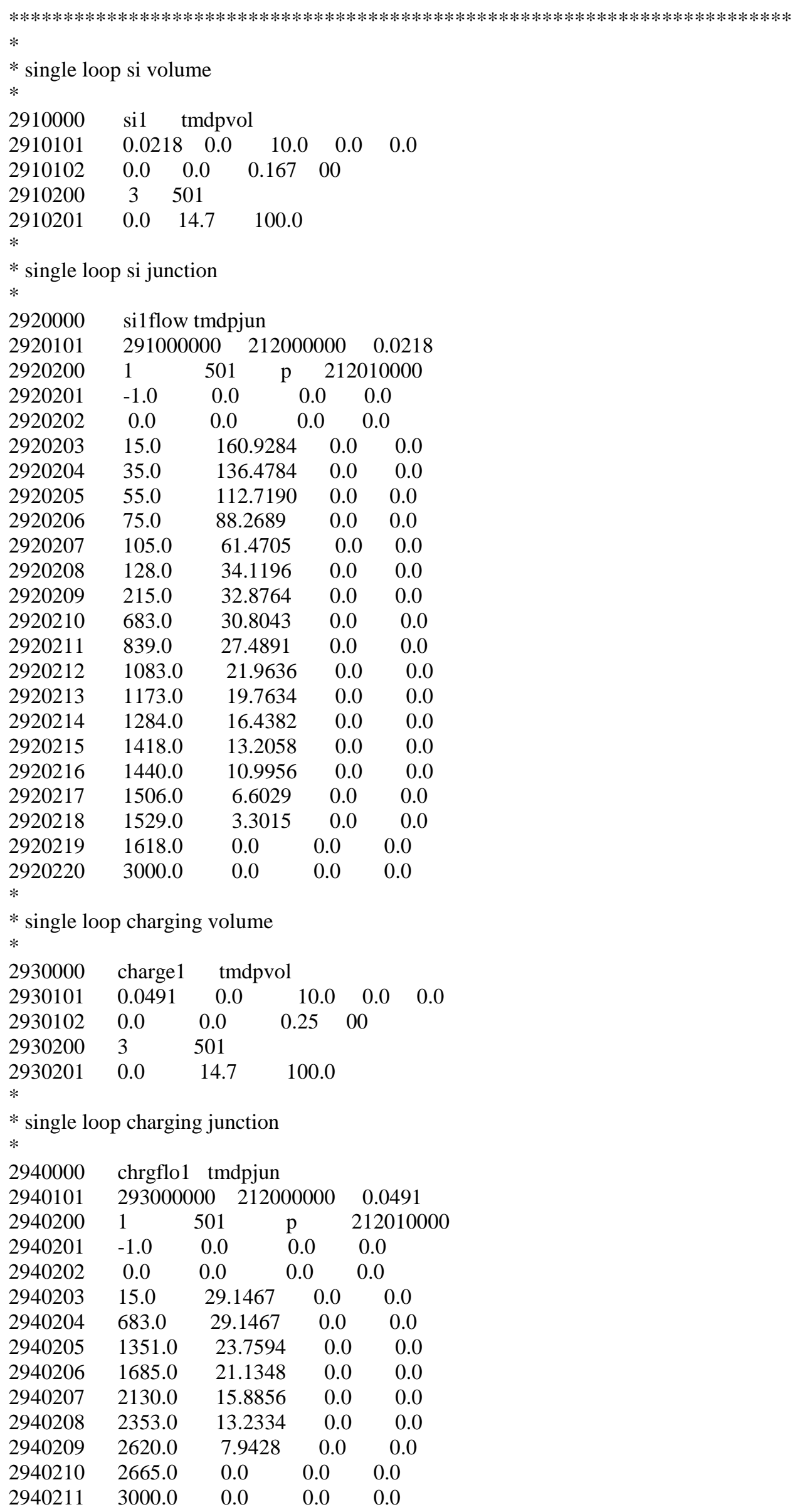




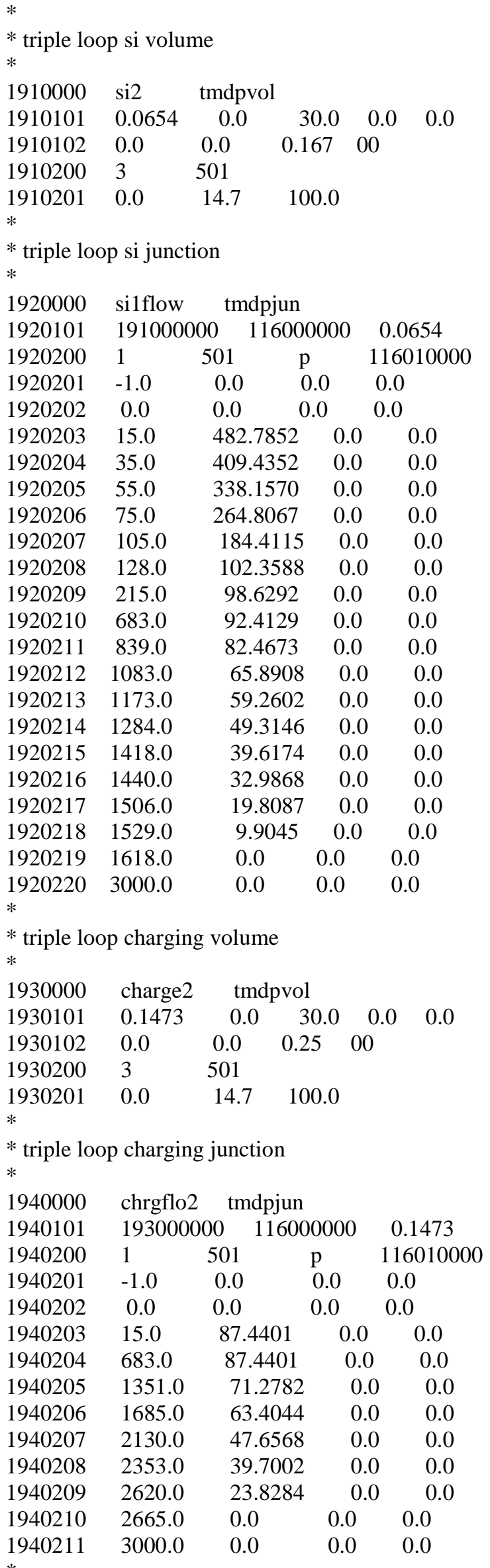




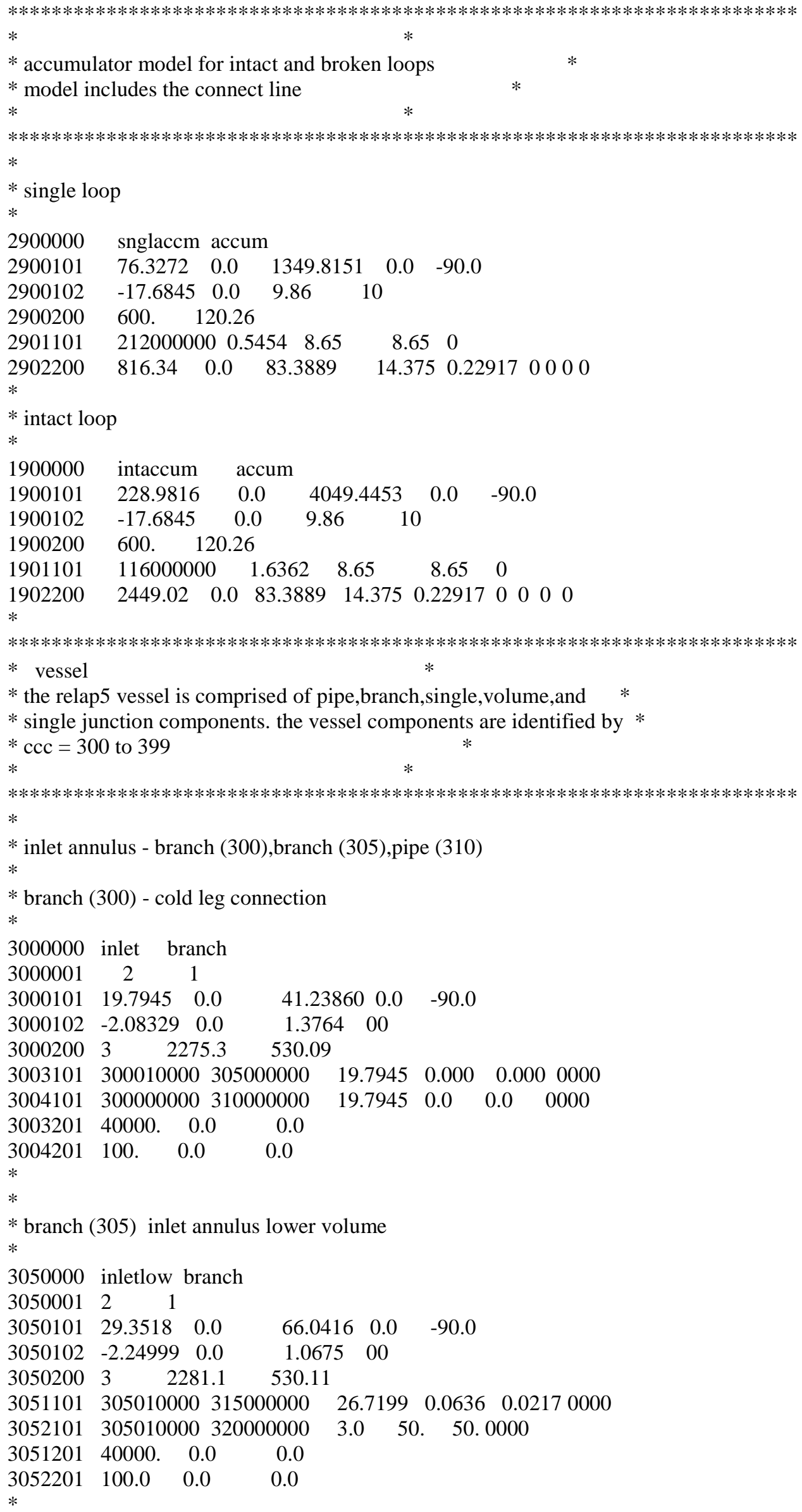


* pipe (310) inlet annulus top volume

3100000 inlettop pipe

31000014

$3100101 \quad 19.7945 \quad 1$

$\begin{array}{lll}3100102 & 32.6417 \quad 2\end{array}$

$\begin{array}{lll}3100103 & 15.4795 \quad 3\end{array}$

$\begin{array}{lll}3100104 & 15.0903 \quad 4\end{array}$

$3100201 \quad 32.6417 \quad 2$

$\begin{array}{lll}3100202 & 15.0903 \quad 3\end{array}$

$\begin{array}{lll}3100301 & 0.0 \quad 4\end{array}$

$\begin{array}{llll}3100401 & 41.2386 & 1\end{array}$

$\begin{array}{lll}3100402 & 80.2442 \quad 2\end{array}$

$\begin{array}{lll}3100403 & 38.0538 \quad 3\end{array}$

$3100404 \quad 22.63545 \quad 4$

$\begin{array}{lll}3100501 & 0.0 \quad 4\end{array}$

$\begin{array}{lll}3100601 & 90.0 \quad 4\end{array}$

$\begin{array}{lll}3100701 & 2.0833 & 1\end{array}$

$\begin{array}{lll}3100702 & 2.4583 & 2\end{array}$

$\begin{array}{lll}3100703 & 2.4583 \quad 3\end{array}$

$3100704 \quad 1.1978 \quad 4$

$\begin{array}{llll}3100801 & 0.0 & 1.3764 & 1\end{array}$

$\begin{array}{llll}3100802 & 0.0 & 1.5417 & 3\end{array}$

$\begin{array}{llll}3100803 & 0.0 & 0.7083 & 4\end{array}$

$\begin{array}{llll}3100901 & 0.0 & 0.0 & 1\end{array}$

$\begin{array}{llll}3100902 & 0.0 & 0.0 & 2\end{array}$

$\begin{array}{llll}3100903 & 6.176 \mathrm{e} 5 & 6.176 \mathrm{e} 5 & 3\end{array}$

$3101001 \quad 00 \quad 4$

$\begin{array}{lll}3101101 & 0000 \quad 2\end{array}$

$31011020000 \quad 3$

$\begin{array}{lllllllll}3101201 & 3 & 2284.1 & 530.09 & 0.0 & 0 & 0 & 1\end{array}$

$\begin{array}{lllllllll}3101202 & 3 & 2283.3 & 530.03 & 0.0 & 0 & 0 & 2\end{array}$

$\begin{array}{lllllllll}3101203 & 3 & 2282.5 & 530.01 & 0.0 & 0 & 0 & 3\end{array}$

$\begin{array}{lllllllll}3101204 & 3 & 2256.2 & 529.99 & 0.0 & 0 & 0 & 4\end{array}$

$3101300 \quad 1$

$\begin{array}{lllll}3101301 & 100 . & 0.0 & 0.0 & 3\end{array}$

********************************************************************************

$*$

* downcomer - pipe (315)

*

3150000 dcomer annulus

31500018

$\begin{array}{lll}3150101 & 26.7199 \quad 8\end{array}$

$\begin{array}{lll}3150301 & 0.0 & 8\end{array}$

$3150401 \quad 57.0560 \quad 1$

$315040258.3376 \quad 5$

$\begin{array}{llll}3150403 & 66.9681 & 6\end{array}$

$\begin{array}{lll}3150404 & 38.9870 & 7\end{array}$

$\begin{array}{llll}3150405 & 89.0654 \quad 8\end{array}$

$\begin{array}{llll}3150501 & 0.0 & 8\end{array}$

$\begin{array}{lll}3150601 & -90.0 & 8\end{array}$

$\begin{array}{llll}3150701 & -2.1353 & 1\end{array}$

$\begin{array}{llll}3150702 & -2.18328 & 5\end{array}$

$\begin{array}{llll}3150703 & -2.50627 & 6\end{array}$

$\begin{array}{llll}3150704 & -1.45900 & 7\end{array}$

$\begin{array}{llll}3150705 & -3.33328 & 8\end{array}$

$\begin{array}{llll}3150801 & 0.0 & 0.630 & 8\end{array}$

$\begin{array}{llll}3150901 & 0.0 & 0.0 & 1\end{array}$

$\begin{array}{llll}3150902 & 0.0 & 0.0000 & 3\end{array}$ 


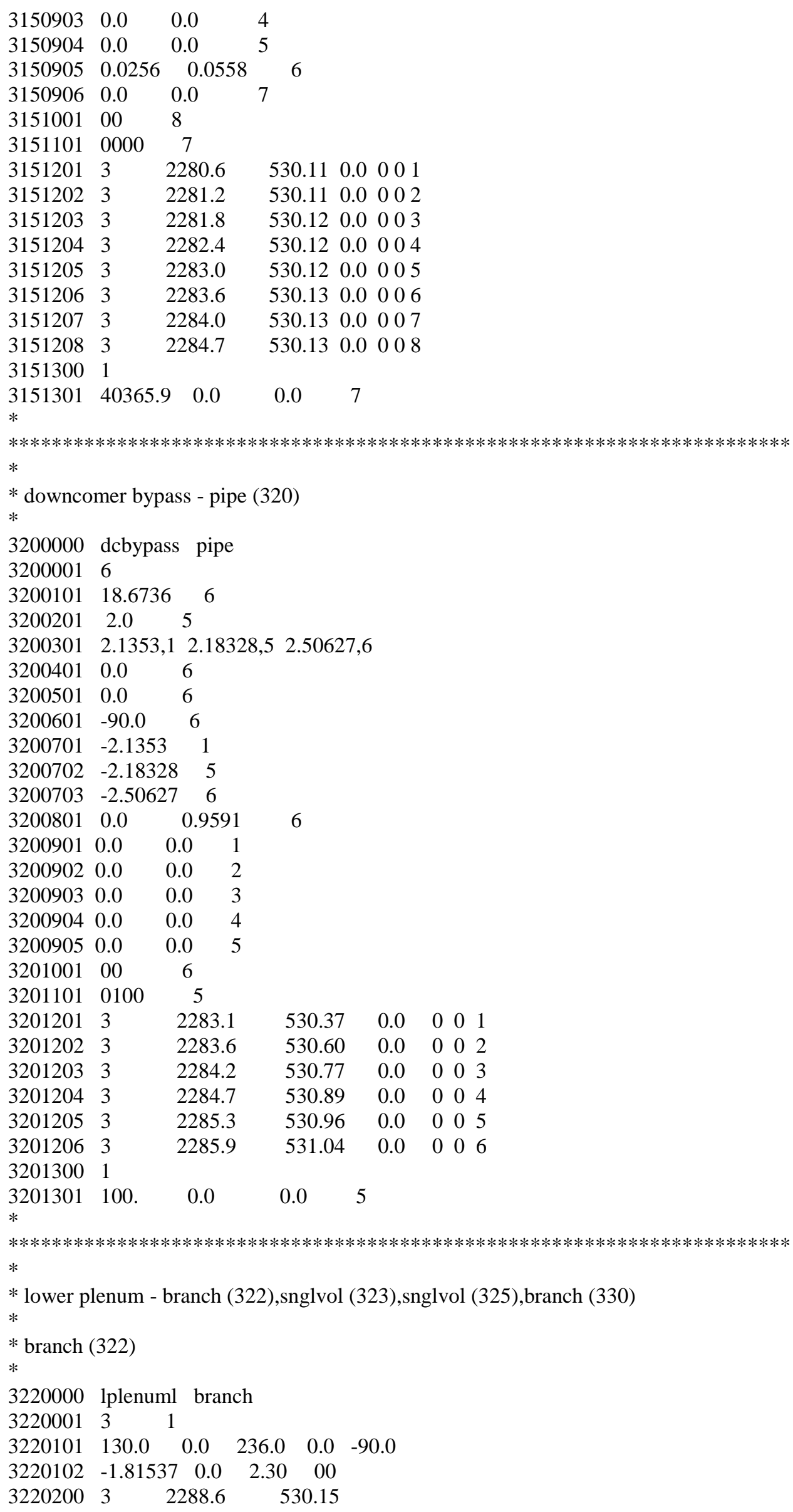




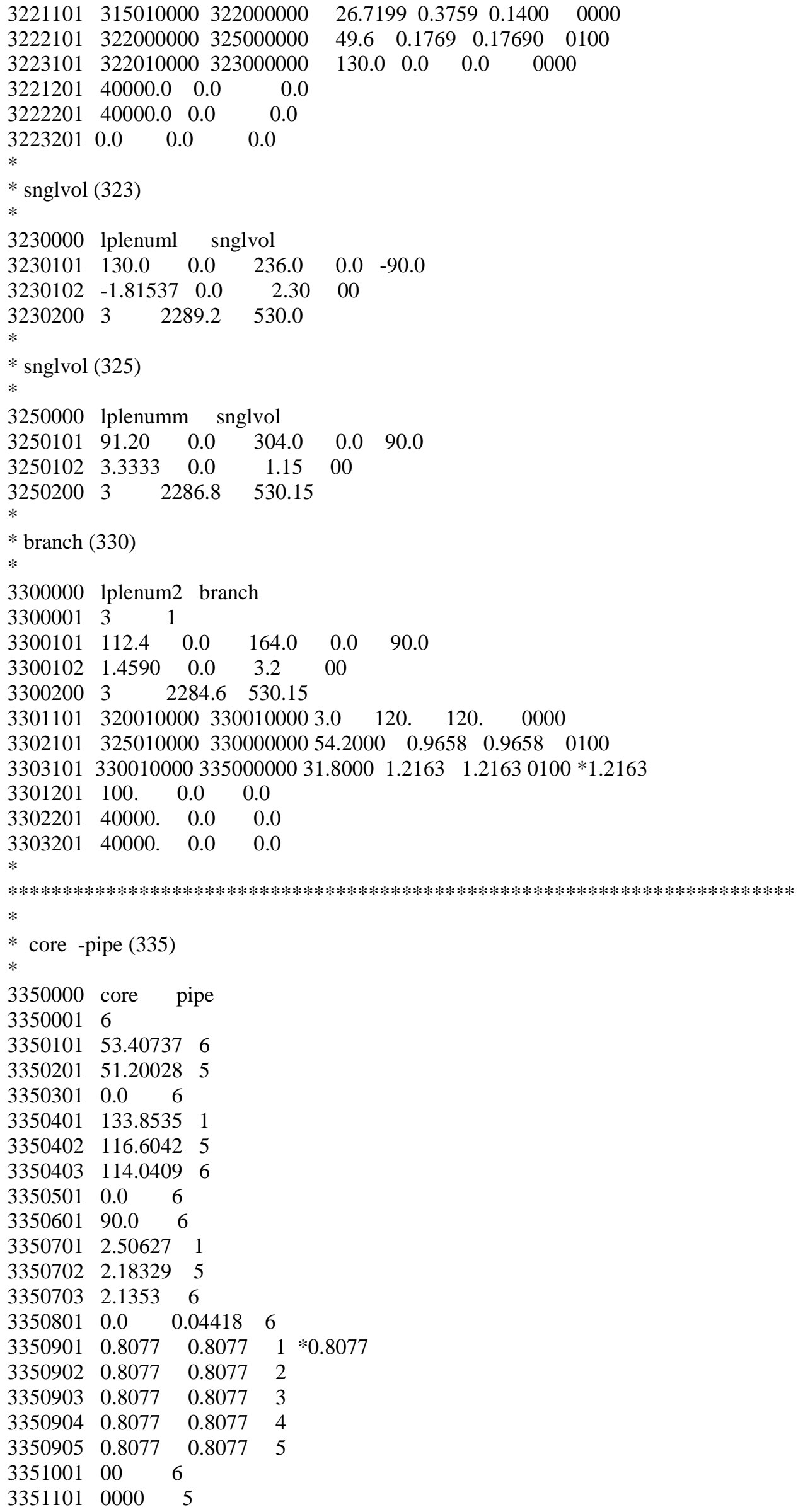




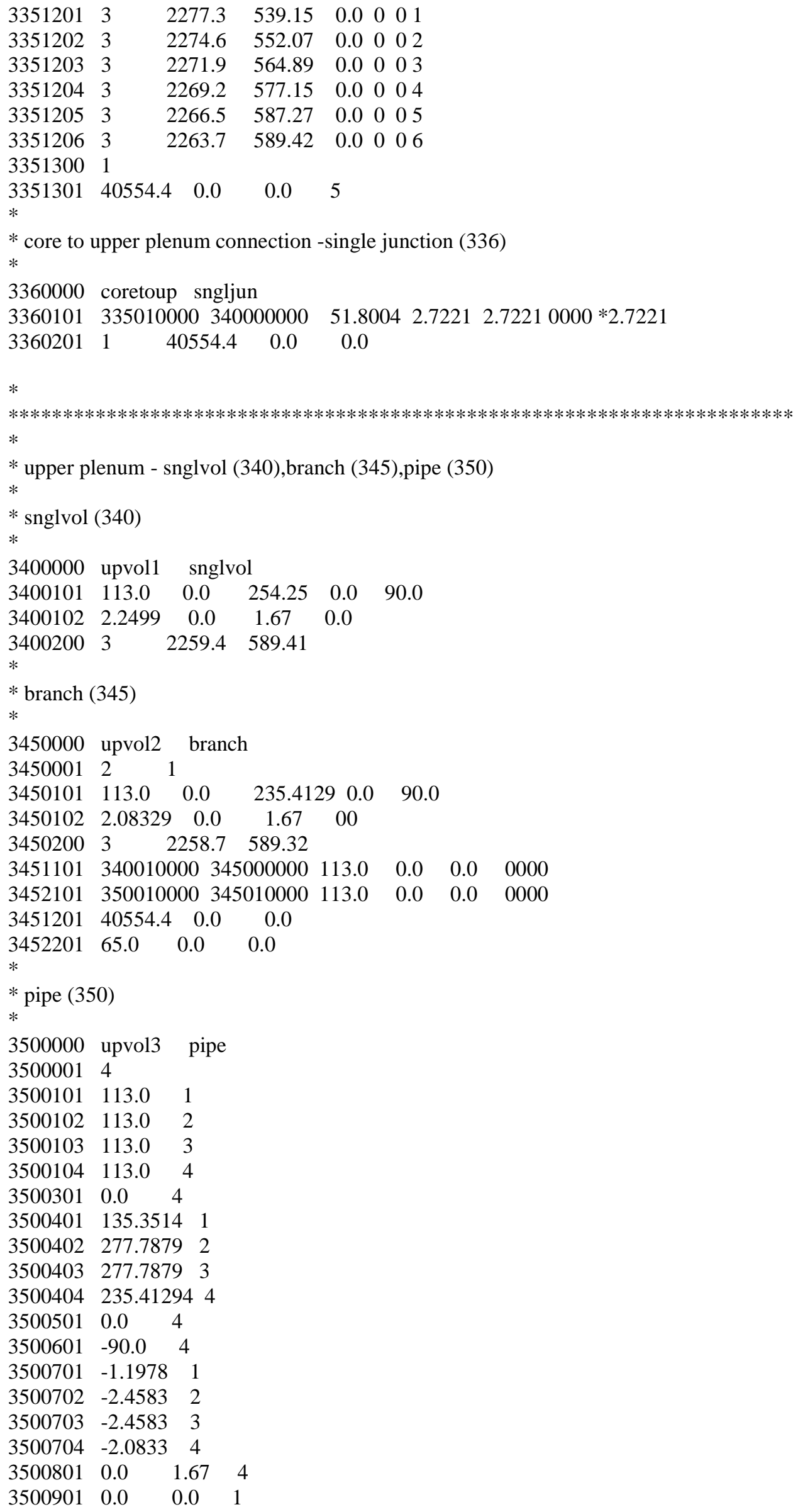




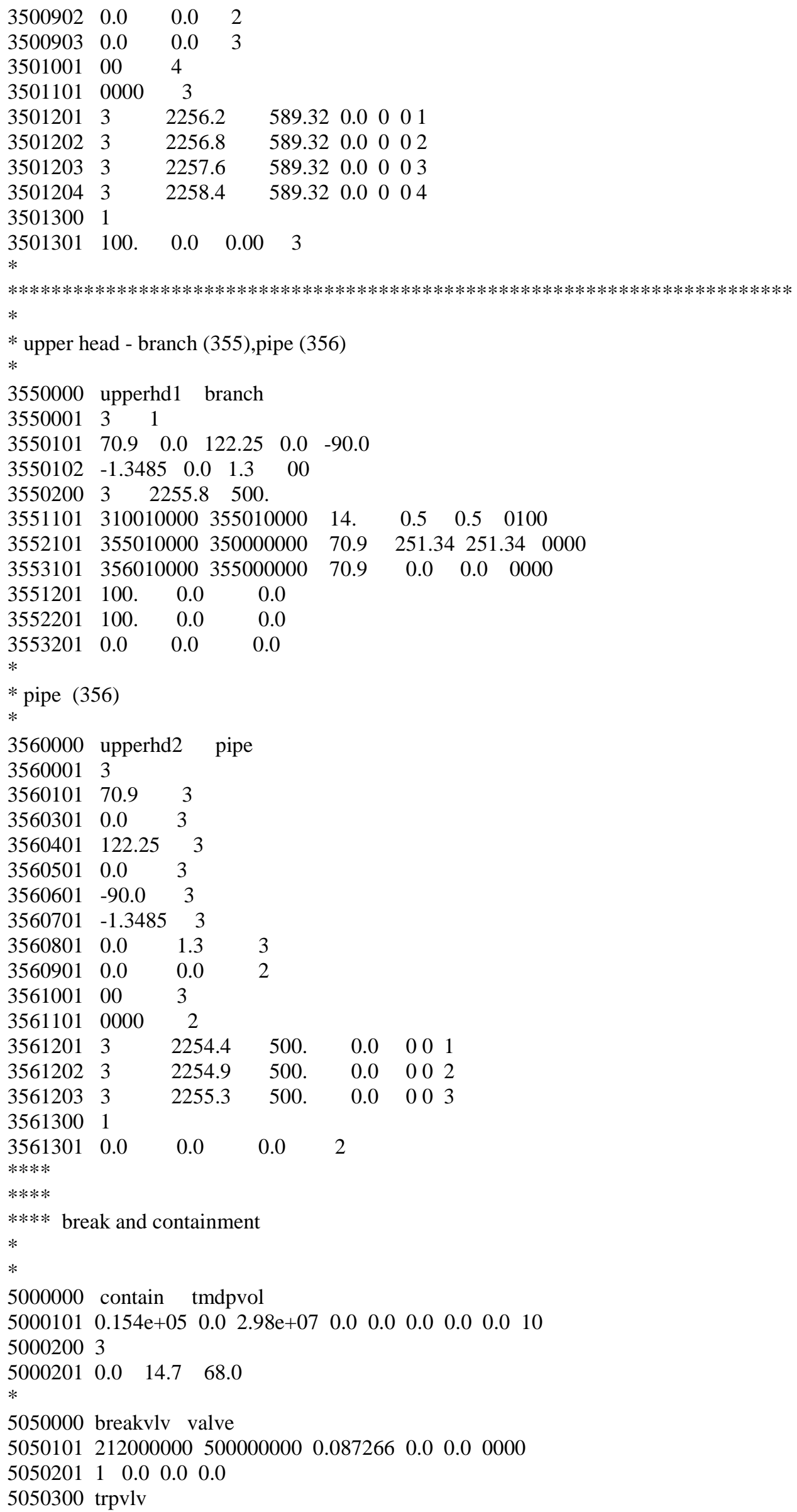




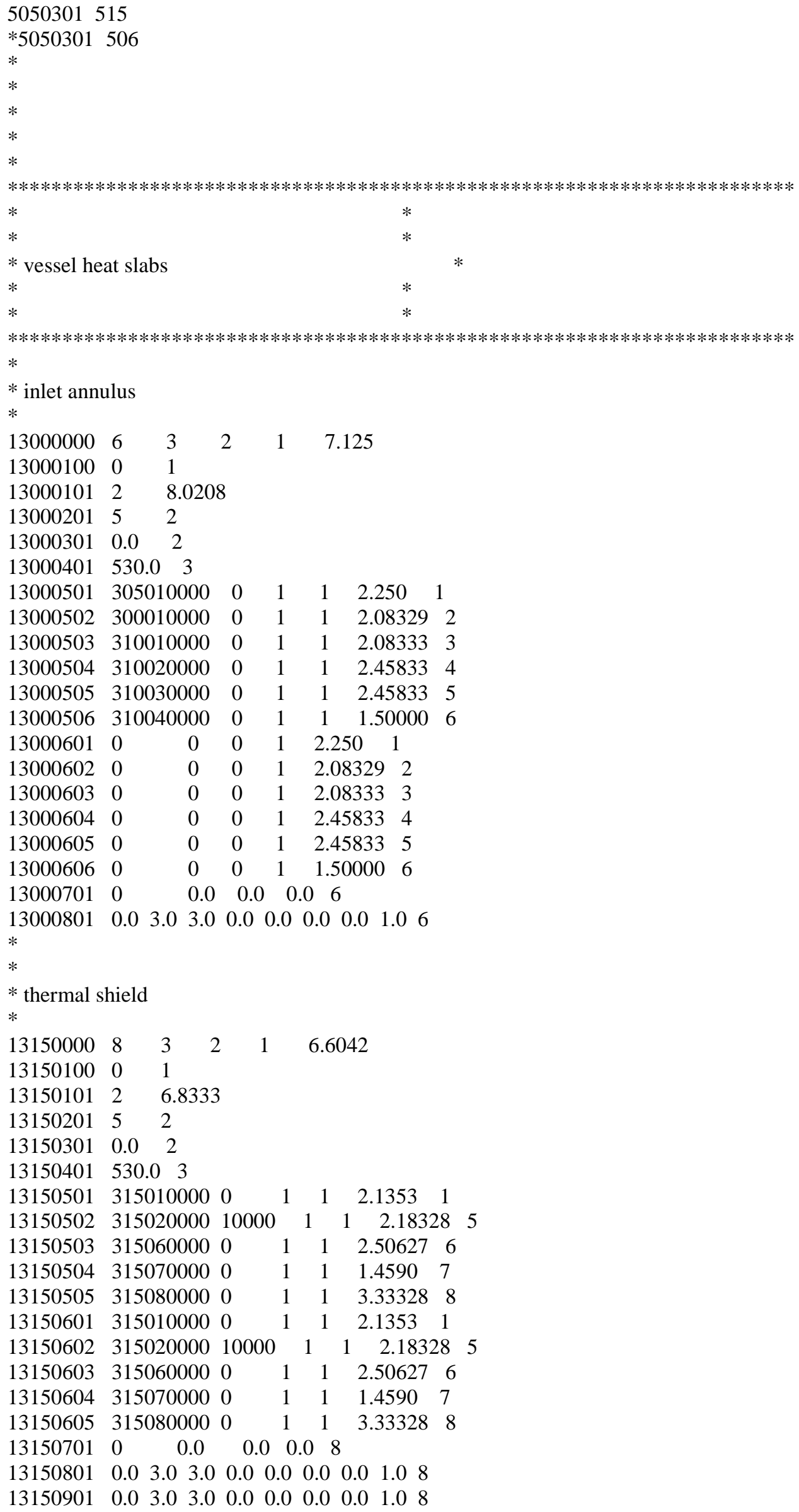




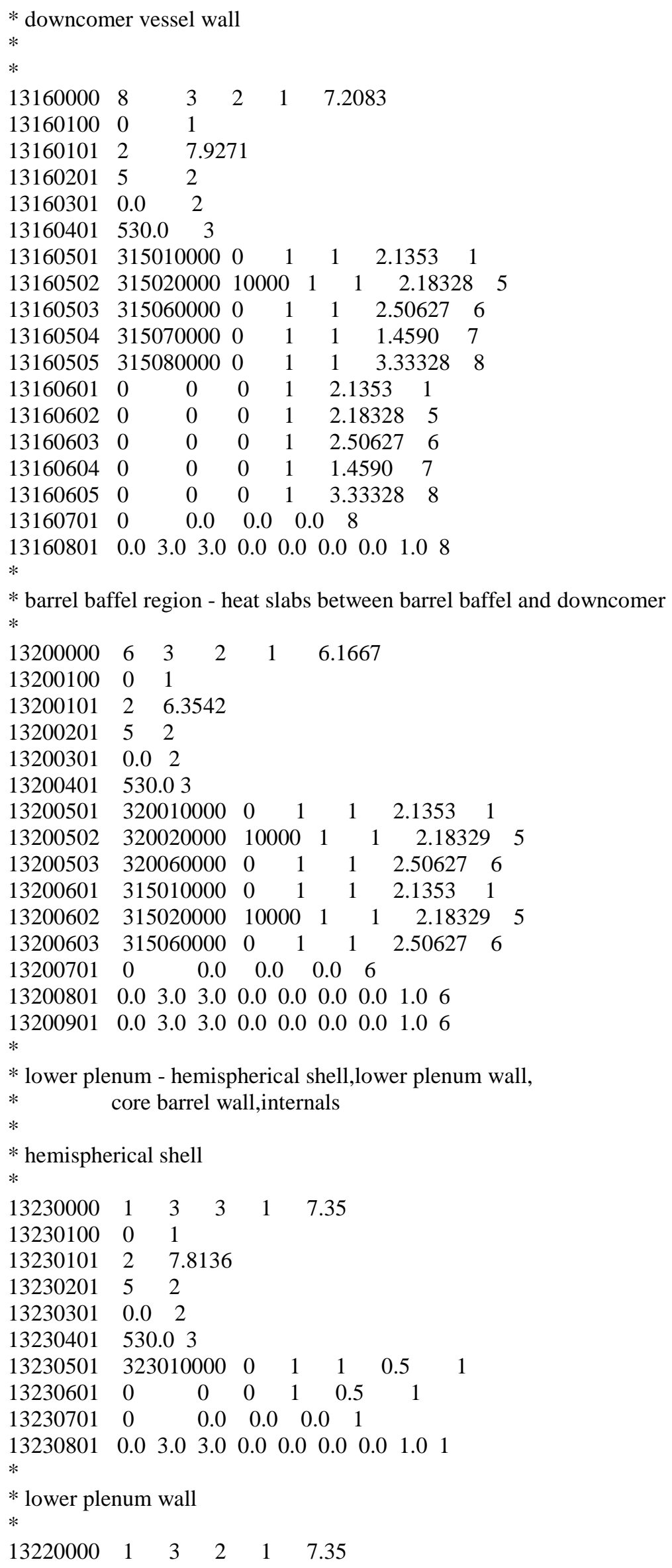




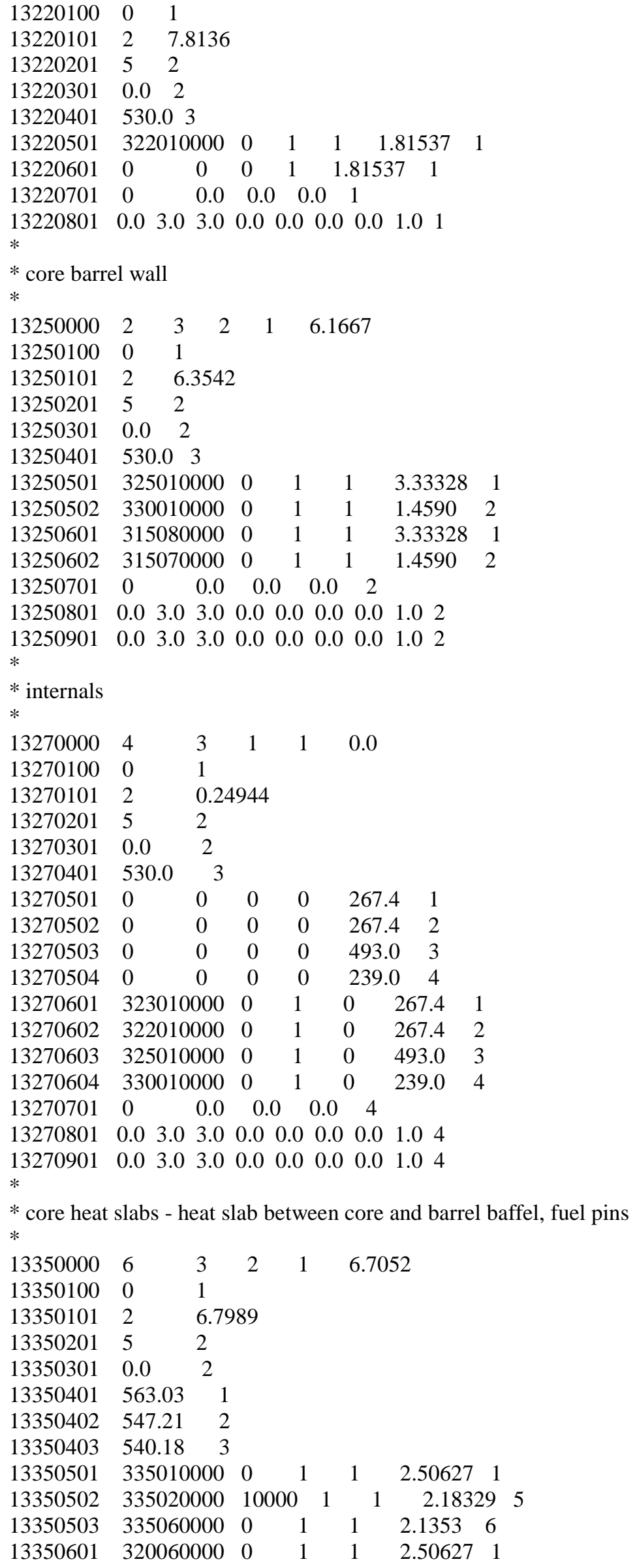




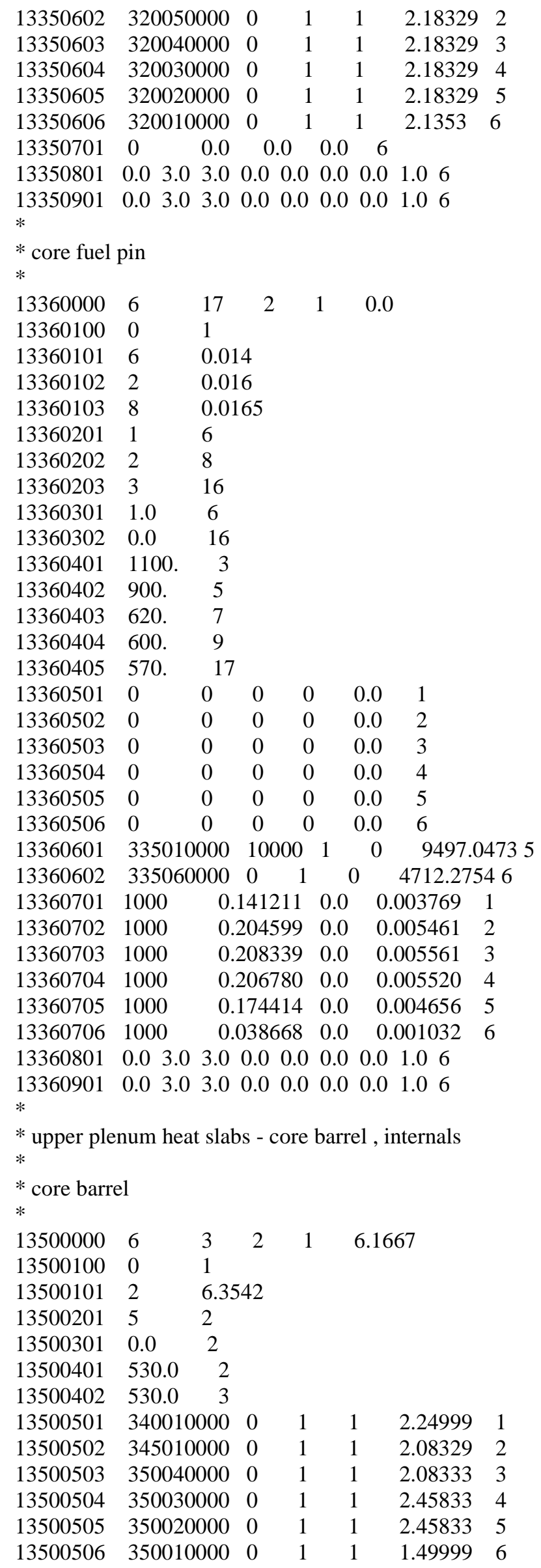




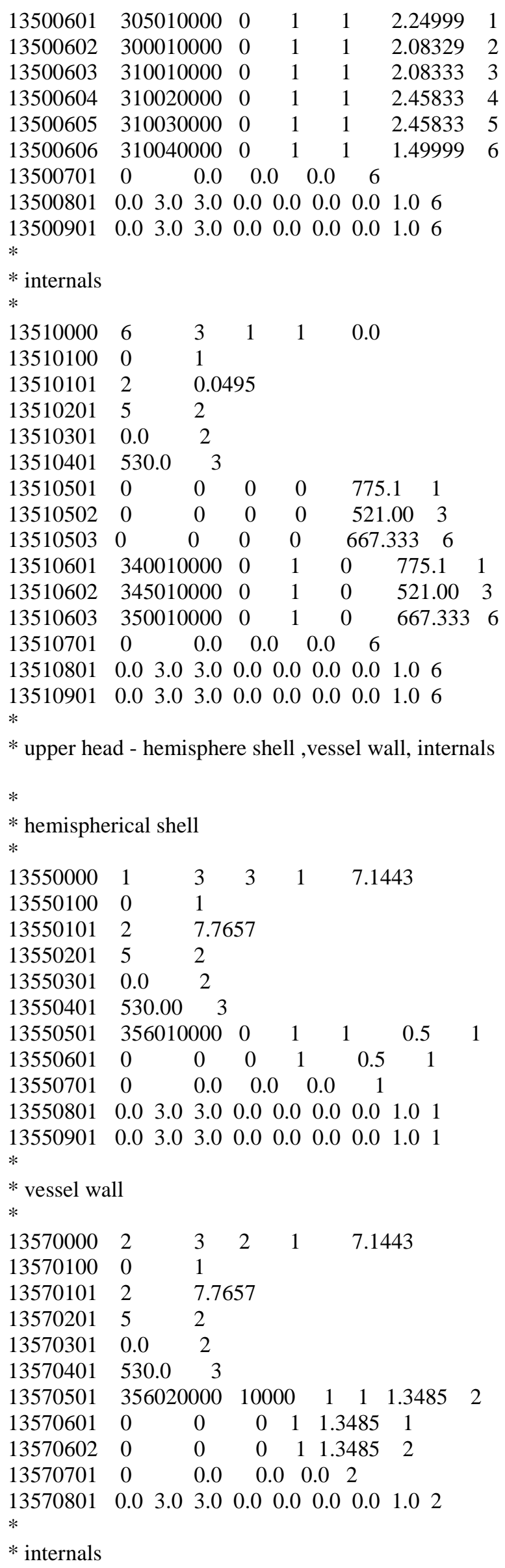




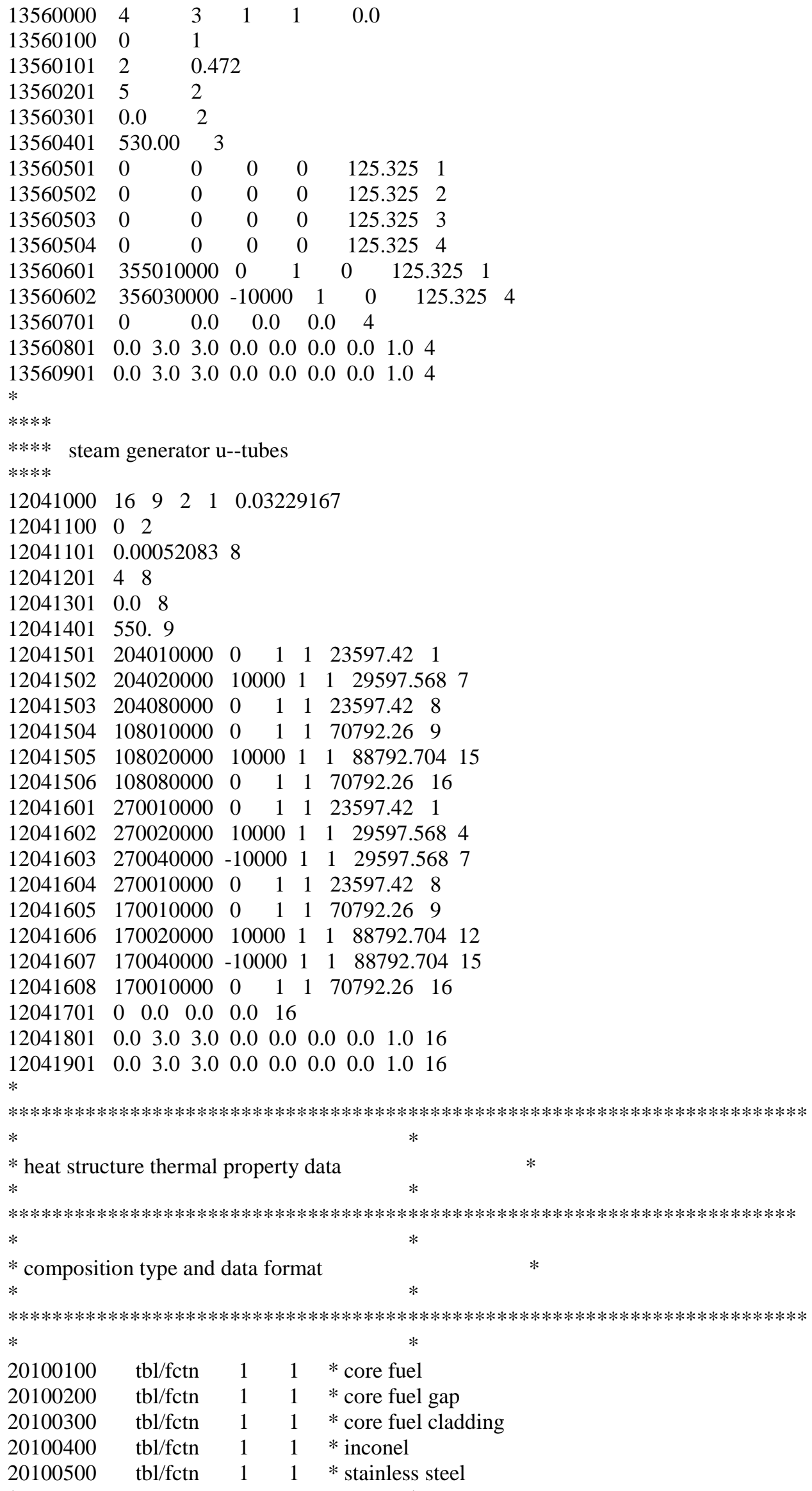




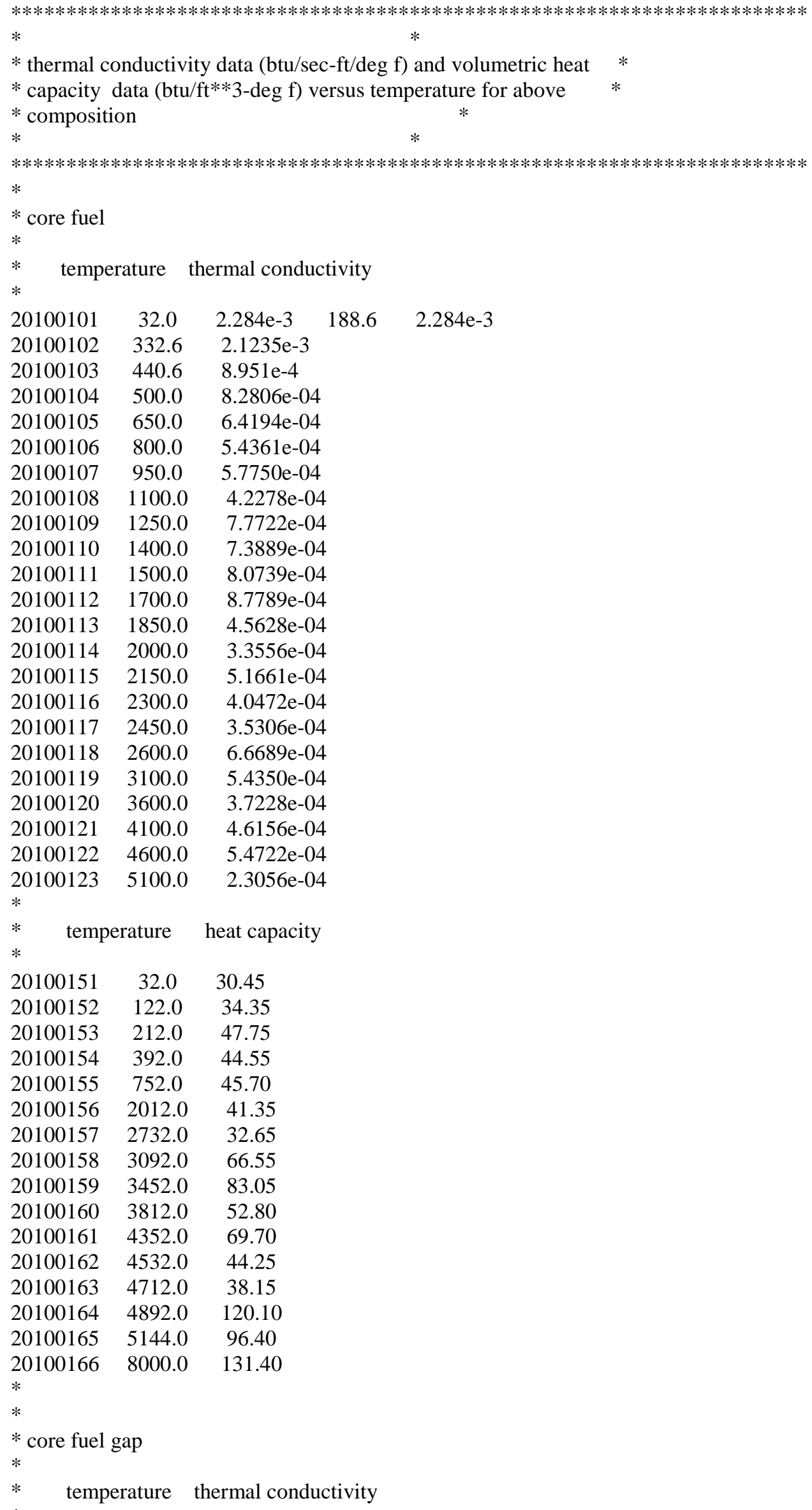




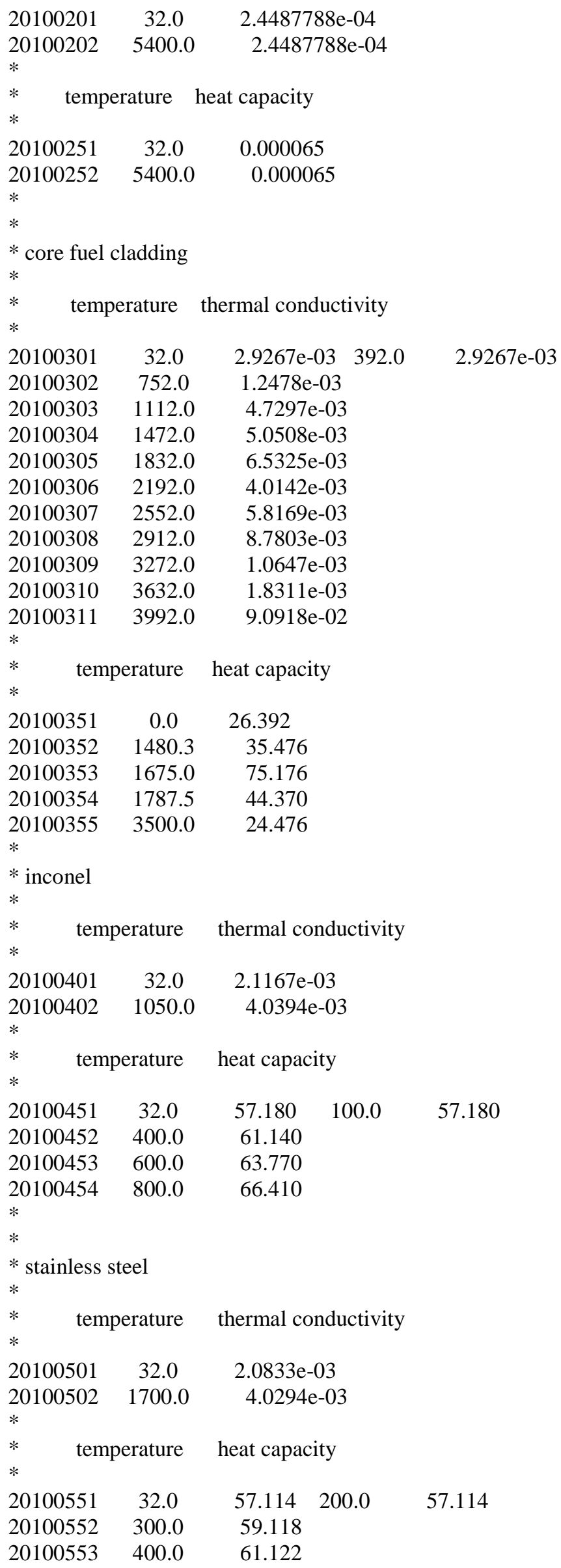




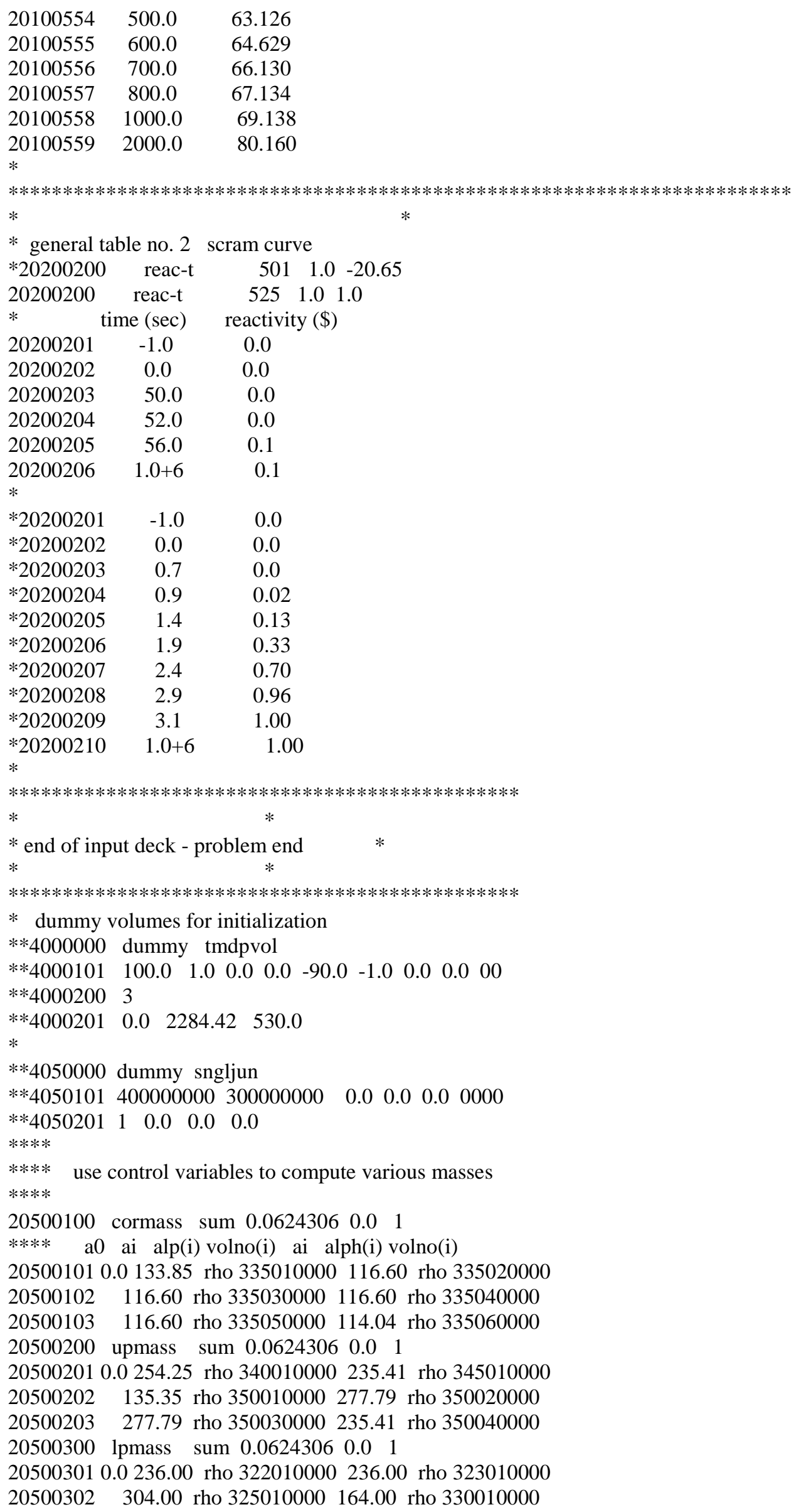


20500400 dnmass sum $0.06243060 .0 \quad 1$

205004010.057 .056 rho 31501000058.338 rho 315020000

2050040258.338 rho 31503000058.338 rho 315040000

$20500403 \quad 58.338$ rho 31505000066.968 rho 315060000

2050040438.987 rho 31507000089.065 rho 315080000

$20500405 \quad 66.042$ rho 30501000041.239 rho 300010000

2050040641.239 rho 31001000080.244 rho 310020000

2050040738.054 rho 31003000022.635 rho 310040000

2050040839.874 rho 32001000040.770 rho 320020000

2050040940.770 rho 32003000040.770 rho 320040000

2050041040.770 rho 32005000046.802 rho 320060000

20500500 uhmass sum $0.06243060 .0 \quad 1$

205005010.0122 .25 rho 355010000122.25 rho 356010000

20500502122.25 rho 356020000122.25 rho 356030000

20500600 ihlmass sum $0.06243060 .0 \quad 1$

205006010.051 .487 rho 10001000060.025 rho 100020000

2050060260.025 rho 10201000096.000 rho 104010000

2050060381.237 rho 104020000

20500700 isgpmass sum $0.0624306 \quad 0.0 \quad 1$

205007010.0290 .88 rho 108010000290.88 rho 108020000

20500702290.88 rho 108030000290.88 rho 108040000

20500703290.88 rho 108050000290.88 rho 108060000

20500704290.88 rho 108070000290.88 rho 108080000

$20500705 \quad 448.29$ rho 106010000448.29 rho 110010000

20500800 iclpsmss sum $0.06243060 .0 \quad 1$

205008010.072 .342 rho 11201000074.967 rho 112020000

$20500802 \quad 105.49$ rho 11203000066.335 rho 112040000

$20500803 \quad 109.09$ rho 112050000

20500900 iclpdmss sum $0.0624306 \quad 0.0 \quad 1$

205009010.0168 .00 rho 11301000093.000 rho 114010000

2050090293.289 rho 11601000093.289 rho 118010000

$20500903 \quad 75.750$ rho 118020000

20501000 presmass sum $0.0624306 \quad 0.0 \quad 1$

205010010.0359 .82 rho 150010000359.82 rho 150020000

20501002449.78 rho 150030000359.82 rho 150040000

$20501003 \quad 179.91$ rho 15005000089.958 rho 150060000

2050100415.924 rho 15201000015.924 rho 152020000

2050100515.924 rho 152030000

20501100 bhlmass sum $0.06243060 .0 \quad 1$

205011010.017 .162 rho 20001000020.008 rho 200020000

2050110220.008 rho 20003000032.000 rho 200040000

2050110327.079 rho 200050000

20501200 bsgpmass sum $0.06243060 .0 \quad 1$

205012010.096 .961 rho 20401000096.961 rho 204020000

2050120296.961 rho 20403000096.961 rho 204040000

2050120396.961 rho 20405000096.961 rho 204060000

2050120496.961 rho 20407000096.961 rho 204080000

20501205149.43 rho 202010000149.43 rho 206010000

20501300 bclpsmss sum $0.06243060 .0 \quad 1$

205013010.024 .114 rho 20801000024.989 rho 208020000

$20501302 \quad 35.162$ rho 20803000022.112 rho 208040000

$20501303 \quad 36.362$ rho 208050000

20501400 bclpdmss sum $0.0624306 \quad 0.0 \quad 1$

205014010.056 .000 rho 20901000031.000 rho 210010000

2050140231.096 rho 21201000031.096 rho 214010000

$20501403 \quad 25.250$ rho 214020000

20501500 isgsmass sum $0.0624306 \quad 0.0 \quad 1$

205015010.01147 .0 rho 1700100001438.6 rho 170020000

205015021438.6 rho 1700300001226.4 rho 170040000

205015031195.2 rho 1700500001250.8 rho 170060000 
205015042132.5 rho 1720100001376.1 rho 174010000

20501505629.23 rho 1760100001590.0 rho 171010000

205015062910.0 rho 1780100001269.9 rho 180010000

20501600 bsgsmass sum $0.06243060 .0 \quad 1$

205016010.0382 .33 rho 270010000479.55 rho 270020000

$20501602 \quad 479.55$ rho 270030000408.79 rho 270040000

$20501603 \quad 398.40$ rho 270050000416.92 rho 270060000

20501604710.82 rho 272010000458.70 rho 274010000

20501605209.74 rho 276010000530.00 rho 271010000

20501606970.00 rho 278010000423.30 rho 280010000

20501700 vesmass sum $1.0 \quad 0.0 \quad 1$

205017010.01 .0 cntrlvar $1 \quad 1.0$ cntrlvar 2

$20501702 \quad 1.0$ cntrlvar $3 \quad 1.0$ cntrlvar 4

$20501703 \quad 1.0$ cntrlvar 5

20501800 ilpmass sum $1.0 \quad 0.0 \quad 1$

205018010.01 .0 cntrlvar $6 \quad 1.0$ cntrlvar 7

$20501802 \quad 1.0$ cntrlvar $8 \quad 1.0$ cntrlvar 9

$20501803 \quad 1.0$ cntrlvar 10

20501900 blpmass sum $1.0 \quad 0.0 \quad 1$

205019010.01 .0 cntrlvar $11 \quad 1.0$ cntrlvar 12

$20501902 \quad 1.0$ cntrlvar $13 \quad 1.0$ cntrlvar 14

20502000 primass sum $1.0 \quad 0.0 \quad 1$

205020010.01 .0 cntrlvar $17 \quad 1.0$ cntrlvar 18

$20502002 \quad 1.0$ cntrlvar 19

20502100 corlev sum $0.018724 \quad 0.0 \quad 1$

$20502101714.29-133.85$ voidg $335010000-116.60$ voidg 335020000

$20502102 \quad-116.60$ voidg $335030000-116.60$ voidg 335040000

$20502103-116.60$ voidg $335050000-114.04$ voidg 335060000

20502200 uplev sum $0.00884960 .0 \quad 1$

$205022011416.0-254.25$ voidg $340010000-235.41$ voidg 345010000

$20502202-135.35$ voidg $350010000-277.79$ voidg 350020000

$20502203-277.79$ voidg $350030000-235.41$ voidg 350040000

\$*************************************************************

$\$ * * * * * * *$ controls de temperatures

$\$ * * * * *$ temperatura aigua del nucli

20502400 tcore sum $0.5 \quad 0.01$

$20502401 \quad 0.01 .0$ tempf $335010000 \quad 1.0$ tempf 335060000

*

20502500 tfuel1 sum 0.058823590 .01

$20502501 \quad 0.01 .0$ httemp $336000101 \quad 1.0$ httemp 336000102

$20502502 \quad 1.0$ httemp 3360001031.0 httemp 336000104

$20502503 \quad 1.0$ httemp 3360001051.0 httemp 336000106

$20502504 \quad 1.0$ httemp $336000107 \quad 1.0$ httemp 336000108

$20502505 \quad 1.0$ httemp 3360001091.0 httemp 336000110

$20502506 \quad 1.0$ httemp $336000111 \quad 1.0$ httemp 336000112

$20502507 \quad 1.0$ httemp 3360001131.0 httemp 336000114

$20502508 \quad 1.0$ httemp 3360001151.0 httemp 336000116

$20502509 \quad 1.0$ httemp 336000117

*

20502600 tfuel2 sum 0.058823590 .01

$20502601 \quad 0.01 .0$ httemp $336000201 \quad 1.0$ httemp 336000202

$20502602 \quad 1.0$ httemp 3360002031.0 httemp 336000204

$20502603 \quad 1.0$ httemp 3360002051.0 httemp 336000206

$20502604 \quad 1.0$ httemp $336000207 \quad 1.0$ httemp 336000208

$20502605 \quad 1.0$ httemp 3360002091.0 httemp 336000210

$20502606 \quad 1.0$ httemp $336000211 \quad 1.0$ httemp 336000212

$20502607 \quad 1.0$ httemp $336000213 \quad 1.0$ httemp 336000214

$20502608 \quad 1.0$ httemp 3360002151.0 httemp 336000216

$20502609 \quad 1.0$ httemp 336000217 
20502700 tfuel3 sum 0.058823590 .01

$20502701 \quad 0.01 .0$ httemp $336000301 \quad 1.0$ httemp 336000302

$20502702 \quad 1.0$ httemp $336000303 \quad 1.0$ httemp 336000304

$20502703 \quad 1.0$ httemp 3360003051.0 httemp 336000306

$20502704 \quad 1.0$ httemp $336000307 \quad 1.0$ httemp 336000308

$20502705 \quad 1.0$ httemp $336000309 \quad 1.0$ httemp 336000310

$20502706 \quad 1.0$ httemp $336000311 \quad 1.0$ httemp 336000312

$20502707 \quad 1.0$ httemp $336000313 \quad 1.0$ httemp 336000314

$20502708 \quad 1.0$ httemp 3360003151.0 httemp 336000316

$20502709 \quad 1.0$ httemp 336000317

*

20502800 tfuel4 sum 0.058823590 .01

$20502801 \quad 0.01 .0$ httemp $336000401 \quad 1.0$ httemp 336000402

$20502802 \quad 1.0$ httemp $336000403 \quad 1.0$ httemp 336000404

$20502803 \quad 1.0$ httemp 3360004051.0 httemp 336000406

$20502804 \quad 1.0$ httemp $336000407 \quad 1.0$ httemp 336000408

$20502805 \quad 1.0$ httemp 3360004091.0 httemp 336000410

$20502806 \quad 1.0$ httemp $336000411 \quad 1.0$ httemp 336000412

$20502807 \quad 1.0$ httemp $336000413 \quad 1.0$ httemp 336000414

$20502808 \quad 1.0$ httemp $336000415 \quad 1.0$ httemp 336000416

$20502809 \quad 1.0$ httemp 336000417

*

20502900 tfuel5 sum 0.058823590 .01

$20502901 \quad 0.01 .0$ httemp $336000501 \quad 1.0$ httemp 336000502

$20502902 \quad 1.0$ httemp $336000503 \quad 1.0$ httemp 336000504

$20502903 \quad 1.0$ httemp 3360005051.0 httemp 336000506

$20502904 \quad 1.0$ httemp $336000507 \quad 1.0$ httemp 336000508

$20502905 \quad 1.0$ httemp $336000509 \quad 1.0$ httemp 336000510

$20502906 \quad 1.0$ httemp $336000511 \quad 1.0$ httemp 336000512

$20502907 \quad 1.0$ httemp $336000513 \quad 1.0$ httemp 336000514

$20502908 \quad 1.0$ httemp $336000515 \quad 1.0$ httemp 336000516

$20502909 \quad 1.0$ httemp 336000517

*

20503000 tfuel6 sum 0.058823590 .01

$20503001 \quad 0.01 .0$ httemp $336000601 \quad 1.0$ httemp 336000602

$20503002 \quad 1.0$ httemp $336000603 \quad 1.0$ httemp 336000604

$20503003 \quad 1.0$ httemp 3360006051.0 httemp 336000606

$20503004 \quad 1.0$ httemp $336000607 \quad 1.0$ httemp 336000608

$20503005 \quad 1.0$ httemp $336000609 \quad 1.0$ httemp 336000610

$20503006 \quad 1.0$ httemp $336000611 \quad 1.0$ httemp 336000612

$20503007 \quad 1.0$ httemp $336000613 \quad 1.0$ httemp 336000614

$20503008 \quad 1.0$ httemp $336000615 \quad 1.0$ httemp 336000616

$20503009 \quad 1.0$ httemp 336000617

$\$ * * * * * * *$ temperatura conbustible

20503100 tfuel sum 0.166670 .01

$20503101 \quad 0.0 \quad 1.0$ cntrlvar $25 \quad 1.0$ cntrlvar 26

$20503102 \quad 1.0$ cntrlvar $27 \quad 1.0$ cntrlvar 28

$20503103 \quad 1.0$ cntrlvar 291.0 cntrlvar 30

$\$ * * * * * * * * * * * * * * * * * * * * * * * * * * * * * * * * * * * * * * * * * * * * * * * * * * * * *$

$\$$ space independent reactor kinetics data

30000000 point

30000001 gamma-ac 3600.e6 $0.0297 .0 \quad 1.0 \quad 0.48$

30000002 ans79-1

300000112

$\begin{array}{lll}30000501 & 45.0 \quad 0.0\end{array}$

$30000601 \quad 1600.0 \quad 0.0$

$\begin{array}{llllll}30000701 & 335010000 & 0 & 0.1367 & -0.00815\end{array}$

$\begin{array}{llllll}30000702 & 335020000 & 0 & 0.2229 & -0.01328\end{array}$

$\begin{array}{llllll}30000703 & 335030000 & 0 & 0.1991 & -0.01187\end{array}$

$\begin{array}{llllll}30000704 & 335040000 & 0 & 0.1862 & -0.01110\end{array}$ 
$\begin{array}{llllll}30000705 & 335050000 & 0 & 0.1922 & -0.01187\end{array}$ $30000706335060000 \quad 0 \quad 0.0629-0.00375$

$\begin{array}{llllll}30000801 & 3360001 & 0 & 0.1367 & -0.0004579\end{array}$

$\begin{array}{llllll}30000802 & 3360002 & 0 & 0.2229 & -0.0007467\end{array}$

$\begin{array}{llllll}30000803 & 3360003 & 0 & 0.1991 & -0.0006670\end{array}$

$\begin{array}{llllll}30000804 & 3360004 & 0 & 0.1862 & -0.0006238\end{array}$

$\begin{array}{llllll}30000805 & 3360005 & 0 & 0.1922 & -0.0006673\end{array}$

$\begin{array}{llllll}30000806 & 3360006 & 0 & 0.0629 & -0.0002107\end{array}$ 